COMPUTER PROGRAM AND DATA LISTING FOR TWO-DIMENSIONAL

GROUND-WATER MODEL FOR LARAMIE COUNTY, WYOMING

By Marvin A. Crist

U.S. GEOLOGICAL SURVEY

Water-Resources Investigations Report 83-4137

Cheyenne, Wyoming

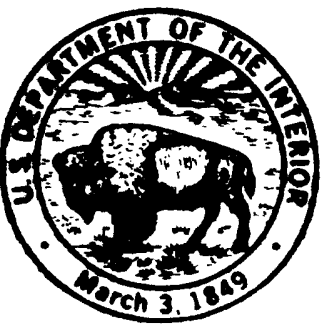


UNITED STATES DEPARTMENT OF THE INTERIOR

JAMES G. WATT, Secretary

GEOLOGICAL SURVEY

Dallas L. Peck, Director

For additional information write to:

District Chief

U.S. Geological Survey

2120 Capitol Avenue

P.0. Box 1125

Cheyenne, Wyoming 82003
Copies of this report can be purchased from:

Open-File Services Section Western Distribution Branch U.S. Geological Survey Box 25425, Federal Center Denver, Colorado 80225 (Telephone: (303) 234-5888) 
Abstract-1

Introduction- 1

Data entry 1

Steady-state simulation-

Transient simulations-

References cited-1. 3

\section{TABLES}

Table 1. Listing of computer program for Laramie County model-....- 4

2. Listing of data for 1920-70 52

3. Listing of data for 1971-77 90 


\section{CONVERSION FACTORS}

For use of readers who prefer to use metric units, conversion factors for the inch-pound units used in this report are listed below:

Multiply

foot

cubic foot

foot per second

cubic foot per second
By

0.3048

0.02832

0.3048

0.02832
To obtain

meter

cubic meter

meter per second

cubic meter per second 


\title{
COMPUTER PROGRAM AND DATA LISTING FOR TWO-DIMENSIONAL \\ GROUND-WATER MODEL FOR LARAMIE COUNTY, WYOMING
}

By Marvin A. Crist

\begin{abstract}
This report is a supplement to the report, "Effect of pumpage on ground-water levels as modeled in Laramie County, Wyoming," published as U.S. Geological Survey Water-Resources Investigations Open-File Report 80-1104 which describes ground-water conditions in post-Cretaceous rocks in Laramie County. The computer program and the data used to model ground-water conditions in post-Cretaceous rocks in Laramie County are listed in this report.
\end{abstract}

\section{INTRODUCTION}

This report is a supplement to the report, "Effect of pumpage on ground-water levels as modeled in Laramie County, Wyoming," (Crist, 1980) which was prepared in cooperation with the Wyoming State Engineer and the Wyoming Department of Economic Planning and Development and describes the ground-water conditions in post-Cretaceous rocks in Laramie County. The purpose of this report is to provide the computer program and model data to hydrologists and water administrators familiar with ground-watermodeling techniques to enable them to make additional model simulations. The general computer program was written by Trescott and others (1976) with a streamflow-accounting procedure added by Hoxie (1977, p. 21) to approximate the interaction between the streams and the aquifer.

A listing of the data for the model is also given in this report. The listing is in the same order and format prescribed by the computer program. Knowledge of Fortran IV program language is necessary to be able to follow the routing and computations made by the computer program.

\section{DATA ENTRY}

The computer program used in this model (table 1) is dated January 1975. The order of entering data is slightly different than in the September 1975 version (Trescott and others, 1976) although documentation for the general program is the same.

A node-identification array (p. 53-56 and p. 91-94) is used to identify the stream nodes and the node where water enters Crow Creek from the Cheyenne municipal treatment plant (Crist, 1980, p. 16). Explanation of the identifying number for each stream follows the node-identification array listing (p. 56 and p. 94) in tables 2 and 3 . The explanation must be followed by a blank card or a card with a zero (as card number 197, table 2). 


\section{STEADY-STATE SIMULATION}

Several assumptions (Crist, 1980, p. 17 and 19) were made in order to simulate steady-state conditions. One assumption was that the hydraulic-head distribution estimated from the potentiometric surface mapped for March 1977 (Crist, 1980, pl. 3) approximates the potentiometric surface during steady-state conditions. All data except aquifer hydraulic conductivity were assumed correct for steady-state conditions; the hydraulic-conductivity distribution was then generated by trial and error. This procedure resulted in a calculated hydraulic-head distribution for steady-state conditions that agrees favorably with the measured hydraulic-head distribution (Crist, 1980, p. 19). The calculated steadystate hydraulic head was used as the initial hydraulic head for the transient simulation.

No attempt was made to verify the hydraulic conductivities generated through trial and error by comparing them with either field measurements of hydraulic conductivity or local geologic conditions. These values, therefore, should not be used to predict local effects of pumping or should be used with caution, as local variations in water levels due to other causes may have resulted in local hydraulic conductivities in the model somewhat different than actually exist. Additional geologic and aquifer-test data are needed to better define the aquifer properties of the units simulated by the Laramie County model.

\section{TRANSIENT SIMULATIONS}

Data for two periods, 1920-70 and 1971-77, are listed in tables 2 and 3. During these periods, pumpage was simulated by the model so that calculated water-level changes could be examined at nodes where water levels were measured 1971-77 (Crist, 1980, p. 21).

An additional transient simulation for $1978-87$ was made with the model. As this was a predictive simulation in which the pumpage was assumed to continue at the same rate as estimated for 1977 (Crist, 1980, p. 23), the data used for this simulation are not included in this report. Any other pumpage rate could have been assumed and be used to simulate conditions beyond 1977 . 


\section{REFERENCES CITED}

Crist, M. A., 1980, Effect of pumpage on ground-water levels as modeled in Laramie County, Wyoming: U.S. Geological Survey Water-Resources Investigations Open-File Report 80-1104, 26 p.

Hoxie, D. T., 1977, Digital model of the Arikaree aquifer near Wheatland, southeastern Wyoming: U.S. Geological Survey Open-File Report $77-676,54 \mathrm{p}$.

Trescott, P. C., Pinder, G. F., and Larson, S. P., 1976, Finitedifference model for aquifer simulation in two dimensions with results of numerical experiments: U.S. Geological Survey Techniques of Water-Resources Investigations, Book 7, Chapter C1, 116 p. 
Table 1.--Listing of computer program for Laramie County model

C

C

C

C

C

C

C

C

C

C

C

C

C

\author{
FINITE-DIFFERENCE MODEL \\ FOR \\ SIMULATION OF GROUND-WATER FLOW \\ IN TWO DIMENSIONS
}

BY P. C. TRESCOTT AND G. F. PINDER

U. S. GEOLOGICAL SURVEY JANUARY, 1975

SOURCE PROGRAM FOR LARAMIE COUNTY MODEL

STEADY-STATE OPTION ADDED 8 DECEMBER 1976

BOUNDARY WELL DISCHARGE/RECHARGE CALCULATED INTERNALLY FROM INITIAL HEAD AND TRANSMISSIVITY DISTRIBUTIONS

\section{STREAMFLOW ACCOUNTING PROCEDURE ADDED 12 APRIL 1977}

NOTE: SUBROUTINE PRNTAI HAS BEEN REMOVED; TO REACTIVATE REPLACE SOURCE DECK AND REMOVE C'S FROM COL 1 OF THE FOLLOWING CARDS: MAN1840\& 1850 , MAN2040, STP $960 \& 970$ A DATA CARD FOR VARIABLE CONTR IS STILL NEEDED, HOWEVER MODIFY STREAM NODE READ-IN PROCEDURE: 25 APRIL 1978
MAN 10

MAN 20

MAN 30

MAN 40

MAN 50

MAN 60

MAN 70

MAN 80

MAN 90

MAN 100

MAN 110

MAN 120

MAN 130

MAN 140

MAN 150

MAN 160

MAN 170

MAN 180

MAN 190

MAN 200

MAN 210

MAN 220

MAN 230

MAN 240

MAN 250

MAN 260

MAN 270

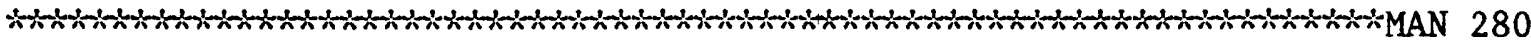

MAIN PROGRAM TO DIMENSION DIGITAL MODEL AND CONTROL SEQUENCE MAN 290 OF COMPUTATIONS

MAN 300

MAN 310

MAN 320

MAN 330

MAN 340

MAN 350

$\$, \mathrm{IU}, \mathrm{JU}$

COMMON /SPARAM/ WATER, CONVRT ,EVAP, CHCK,PNCH, NUM, HEAD, CONTR, EROR, LEMAN 360 1 AK, RECH , SIP , U , SS , TT , TMIN , ETDIST , QET , ERR, TMAX, CDLT , HMAX, YDIM, WIDTH , MAN 370 2NUMS , LSOR , ADI ,DELT , SUM, SUMP , SUBS , STORE, TEST , ETQB , ETQD , FACTX, FACTY , MAN 380 3 IERR , KOUNT , IF INAL , NUMT , KT , KP , NPER , KTH, ITMAX, LENGTH , NWEL , NW , DIML ,DIMAN 390 4MW, JNO 1 , INO1 , R , P , PU , I , J , NODE , STDY , KPH , IQPNC

MAN 400 COMMON /STREAM/ NRIV,NRPR, NODRV (20), NDR (20), NMRV $(20), Q R I V(3,20)$,

$\$ \operatorname{IRUP}(20), \operatorname{JRUP}(20), \operatorname{IRDN}(20), \operatorname{JRDN}(20)$

MAN 410

MAN 420

MAN 430

MAN 440

MAN 450

MAN 460

MAN 470

REAL $\div 4 \mathrm{KEEP}, \mathrm{M}, \mathrm{HEADNG}(32)$

REAL *8PHI , G, BE, TEMP , Z

INTEGER R, P, PU, DIMI ,DIMW , CHK, WATER , CONVRT , EVAP , CHCK, PNCH, NUM, HEAD , MAN 480 ICONTR , LEAK, RECH , SIP , ADI , NODE , STDY

MAN 490

MAN 500

MAN 510

CALL ERRSET $(208,256,-1,1)$

---READ TITLE, PROGRAM OPTIONS AND PROGRAM SIZE---

MAN 550 
Table 1.--Listing of computer program for Laramie County mode1--Continued

WRITE $(P, 330)$ HEADNG

MAN 560

READ ( R, 350) WATER, LEAK, CONVRT, EVAP, RECH, NUMS , CHCK, PNCH, NUM, HEAD MAN 570

$\$, N O D E$, STDY

MAN 580

WRITE (P, 360) IVATER, LEAK, CONVRT , EVAP, RECH, NUMS , CHCK, PNCH, NUM, HEAD MAN 590

$\$$, NODE , STDY

MAN 600

IF (NUMS.EQ.CHK(11).OR.NUMS.EQ.CHK(12).OR.NUMS.EQ.CHK(13)) GO TO 2MAN 610 10

WRITE $(P, 320)$

STOP

20 READ $(R, 290)$ DIML, DIMW, NW

WRITE $(P, 310)$ DIML, DIMW, NW

MAN 620

MAN 630

MAN 640

MAN 650

MAN 660

C

---COMPUTE DIMENSIONS FOR ARRAYS---

MAN 670

$\mathrm{I} Z=\mathrm{DIML}$

$\mathrm{JZ}=\mathrm{DIMW}$

$\mathrm{IH}=\operatorname{MAXO}(1, \mathrm{NW})$

IMAX=MAXO (DIML, DIMW)

ISIZ $=D I M L \div D I M W$

ISUM $=2 *$ ISIZ +1

$\mathrm{L}(1)=1$

DO $30 \quad I=2,4$

L(I) $=$ ISUM

30 ISUM $=$ ISUM $+2 *$ IMAX

DO $40 \quad I=5,16$

$\mathrm{L}(\mathrm{I})=$ ISUM

40 ISUM=ISUM+ISIZ

IF (WATER.NE.CHK(2)) GO TO 60

DO $50 \quad I=17,19$

$\mathrm{L}(\mathrm{I})=\mathrm{ISUM}$

50 ISUM=ISUM+ISIZ

IP=DIML

$\mathrm{JP}=\mathrm{DIMW}$

GO TO 80

60 DO $70 \quad I=17,19$

$L(I)=I S U M$

70 ISUM $=$ ISUM +1

$\mathrm{IP}=1$

$\mathrm{JP}=1$

80 IF (LEAK.NE.CHK(9)) GO TO 100

DO $90 \quad I=20,22$

$\mathrm{L}(\mathrm{I})=\mathrm{ISUM}$

90 ISUM=ISUM+ISIZ

IR=DIML

$\mathrm{JR}=\mathrm{DIMW}$

GO TO 120

MAN 680

MAN 690

MAN 700

MAN 710

MAN 720

MAN 730

MAN 740

MAN 750

MAN 760

MAN 770

MAN 780

MAN 790

MAN 800

MAN 810

MAN 820

MAN 830

MAN 840

MAN 850

MAN 860

MAN 870

MAN 880

MAN 890

MAN 900

MAN 910

MAN 920

MAN 930

MAN 940

MAN 950

MAN 960

MAN 970

MAN 980

MAN 990

MAN 1000

100 DO $110 \quad \mathrm{I}=20,22$

MAN 1010

$\mathrm{L}(\mathrm{I})=\mathrm{ISUM}$

MAN1020

MAN1030

110 ISUM $=$ ISUM+1

IR $=1$

$\mathrm{JR}=1$

MAN 1040

MAN 1050

120 IF (CONVRT.NE.CHK(7)) GO TO 130

MAN1060

$\mathrm{L}(23)=$ ISUM

MAN1070

ISUM=ISUM+ISIZ

MAN 1080

IC=DIML

MAN 1090

$\mathrm{JC}=\mathrm{DIMW}$

MAN1 100 
Table 1.--Listing of computer program for Laramie County model--Continued

GO TO 140

MAN 1110

$130 \mathrm{~L}(23)=\mathrm{ISUM}$

MAN1 120

ISUM=ISUM+1

MAN 1130

IC $=1$

$\mathrm{JC}=1$

140 IF (EVAP.NE.CHK(6)) GO TO 150

$\mathrm{L}(24)=$ ISUM

ISUM=ISUM+IS IZ

MAN 1140

II $=$ DIML

$\mathrm{JL}=\mathrm{DIMW}$

MAN 1150

MAN 1160

MAN 1170

MAN 1180

MAN 1190

GO TO 160

MAN 1200

MAN 1210

$150 \mathrm{~L}(24)=$ ISUM

MAN 1220

ISUM=ISUM+ 1

MAN 1230

IL $=1$

MAN 1240

$\mathrm{JL}=1$

MAN 1250

160 IF (NUMS.NE.CHK(11)) GO TO 180

MAN 1260

DO $170 \quad I=25,28$

$\mathrm{L}(\mathrm{I})=\mathrm{ISUM}$

MAN 1270

MAN 1280

170 ISUM=ISUM+ISIZ

MAN 1290

IS $=$ DIML

MAN 1300

JS $=$ DIMW

MAN 1310

GO TO 200

MAN 1320

180 DO $190 \mathrm{I}=25,28$

$L(I)=I S U M$

MAN 1330

MAN 1340

190 ISUM $=$ ISUM +1

IS $=1$

$\mathrm{JS}=1$

200 DO $210 \quad I=29,31$

$\mathrm{L}(\mathrm{I})=$ ISUM

210 ISUM $=$ ISUM+DIMW

DO $220 \mathrm{I}=32,33$

$\mathrm{L}(\mathrm{I})=\mathrm{ISUM}$

220 ISUM=ISUM+DIML

$\mathrm{L}(34)=$ ISUM

ISUM=ISUM + IH

$\mathrm{L}(35)=$ ISUM

ISUM $=I S U M+2 * I H$

IF (NODE.NE.CHK(14)) GO TO 2200

MAN 1350

MAN 1360

MAN 1370

MAN 1380

MAN 1390

MAN 1400

MAN 1410

MAN 1420

MAN 1430

MAN 1440

MAN 1450

MAN 1460

MAN 1470

DO $2201 \mathrm{I}=36,37$

MAN 1480

L(I) $=$ ISUM

MAN 1490

MAN 1500

MAN 1510

MAN 1520

IU=DIML

MAN 1530

JU=DIMW

MAN 1540

GO TO 2210

2200 DO $2205 \mathrm{I}=36,37$

MAN 1550

$\mathrm{L}(\mathrm{I})=$ ISUM

MAN 1560

MAN 1570

MAN 1580

MAN 1590

MAN 1600

MAN 1610

MAN 1620

MAN 1630

MAN 1640

C ---PASS DIMENSIONS OF PROBLEM TO SUBROUTINES---

CALL DATAI ( $(\mathrm{L}(1)), \mathrm{Y}(\mathrm{L}(7)), \mathrm{Y}(\mathrm{L}(8)), \mathrm{Y}(\mathrm{L}(9)), \mathrm{Y}(\mathrm{L}(10)), \mathrm{Y}(\mathrm{L}(11)), \mathrm{Y}(\mathrm{L}($ M MAN 1650 
Table 1.--Listing of computer program for Laramie County mode1--Continued

12)) , Y(L(13)) , Y(L(14)), Y(L(15)), Y(L(16)), Y(L(17)), Y(L(18)), Y(L(19)) MAN1660 $2, Y(L(20)), Y(L(21)), Y(L(22)), Y(L(23)), Y(L(24)), Y(L(29)), Y(L(32)), Y($ MAN1670 $3 \mathrm{~L}(34)), \mathrm{Y}(\mathrm{L}(35)), \mathrm{Y}(\mathrm{L}(36)), \mathrm{Y}(\mathrm{L}(37)))$ MAN 1680

CALL STEP(Y(L(1)), Y(L(5)), Y(L(7)), Y(L(8)), Y(L(9)), Y(L (14)), Y(L(17) MAN 1690

1) $, Y(L(18)), Y(L(23)), Y(L(29)), Y(L(30)), Y(L(32)), Y(L(34)), Y(L(35))$, MAN1700 $\$ Y(L(36)), Y(L(37)))$ MAN 1710

IF (NUMS.EQ.CHK(11)) CALL SOLVE $1(\mathrm{Y}(\mathrm{L}(1)), \mathrm{Y}(\mathrm{L}(2)), \mathrm{Y}(\mathrm{L}(3)), \mathrm{Y}(\mathrm{L}(4))$, YMAN 1720 $1(\mathrm{~L}(5)), \mathrm{Y}(\mathrm{L}(6)), \mathrm{Y}(\mathrm{L}(7)), \mathrm{Y}(\mathrm{L}(9)), \mathrm{Y}(\mathrm{L}(12)), \mathrm{Y}(\mathrm{L}(13)), \mathrm{Y}(\mathrm{L}(14)), \mathrm{Y}(\mathrm{L}(15))$ MAN 1730 $2, Y(L(16)), Y(L(25)), Y(L(26)), Y(L(27)), Y(L(28)), Y(L(29)), Y(L(31)), Y($ MAN 1740 $3 \mathrm{~L}(32)), \mathrm{Y}(\mathrm{L}(33)), \mathrm{Y}(\mathrm{L}(8)), \mathrm{Y}(\mathrm{L}(23)), \mathrm{Y}(\mathrm{L}(36)))$

MAN 1750

CALL COEF (Y(L(1)), Y(L(5)), Y(L(6)), Y(L(7)), Y(L(8)), Y(L(9)), Y(L(10)) MAN1760 $1, \mathrm{Y}(\mathrm{L}(11)), \mathrm{Y}(\mathrm{L}(12)), \mathrm{Y}(\mathrm{L}(14)), \mathrm{Y}(\mathrm{L}(15)), \mathrm{Y}(\mathrm{L}(16)), \mathrm{Y}(\mathrm{L}(17)), \mathrm{Y}(\mathrm{L}(18)), \mathrm{Y}$ (MAN1770 $2 \mathrm{~L}(19)), \mathrm{Y}(\mathrm{L}(20)), \mathrm{Y}(\mathrm{L}(21)), \mathrm{Y}(\mathrm{L}(22)), \mathrm{Y}(\mathrm{L}(23)), \mathrm{Y}(\mathrm{L}(24)), \mathrm{Y}(\mathrm{L}(29)), \mathrm{Y}(\mathrm{L}$ (3MAN1780 32)), Y(L(36)), Y(L(37)))

MAN 1790

CALL CHECKI (Y(L (1)) , Y(L(5)) , Y(L(6)) , Y(L(7)) , Y(L (9)) , Y(L(10)) , Y (L (1MAN 1800

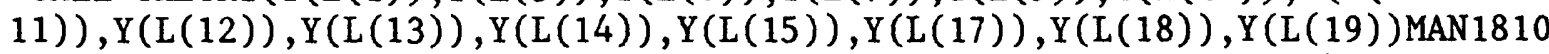
$2, \mathrm{Y}(\mathrm{L}(20)), \mathrm{Y}(\mathrm{L}(21)), \mathrm{Y}(\mathrm{L}(22)), \mathrm{Y}(\mathrm{L}(23)), \mathrm{Y}(\mathrm{L}(24)), \mathrm{Y}(\mathrm{L}(29)), \mathrm{Y}(\mathrm{L}(32)) \cdot$ MAN1820 $3, \mathrm{Y}(\mathrm{L}(36)))$

MAN 1830

C CALL PRNTAI $(\mathrm{Y}(\mathrm{L}(1)), \mathrm{Y}(\mathrm{L}(8)), \mathrm{Y}(\mathrm{L}(9)), \mathrm{Y}(\mathrm{L}(12)), \mathrm{Y}(\mathrm{L}(14)), \mathrm{Y}(\mathrm{L}(29)), \mathrm{Y}($ LMAN1840

MAN 1850

C

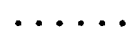

$$
\mathrm{KT}=0
$$

IFINAL $=0$

IF (WATER.EQ.CHK(2)) CALL TRANS

\section{CALL CHECK}

---COMPUTE ITERATION PARAMETERS---

IF (NUMS.EQ.CHK(11)) CALL ITER1

\section{---INITIALIZE PARAMETERS FOR ALPHAMERIC MAP---}

IF (CONTR.EQ.CHK(3)) CALL MAP

---COMPUTE T COEFFICIENTS FOR ARTESIAN PROBLEM---

IF (WATER.NE.CHK(2)) CALL TCOF

MAN 1880

MAN 1890

MAN 1900

MAN 1910

MAN1920

MAN 1930

MAN 1940

MAN 1950

MAN 1960

MAN 1970

MAN 1980

MAN 1990

MAN2000

MAN2010

MAN2020

MAN2030

MAN2040

MAN2050

MAN2060

MAN2070

MAN2080

MAN2090

---COMPUTE MASS BALANCE USING INPUT DATA---

MAN2 100

IFINAL $=1$

MAN2 110

CALL CHECK

MAN2 120

IF INAL $=0$

MAN2 130

MAN2 140

C

C 230 CALL NEWPER

MAN2 160

MAN2 170

$\mathrm{KT}=0$

MAN 2180

IFINAL $=0$

MAN2 190

IERR $=0$

MAN2200 
Table 1.--Listing of computer program for Laramie County model--Continued

C

C ---START NEW TIME STEP COMPUTATIONS---

C

C ---COMPUTE TRANSIENT PART OF LEAKAGE TERM---

IF (LEAK.EQ.CHK(9).AND.SS.NE.0.) CALL CLAY

C

C

250 CONTINUE

IF (NUMS.EQ.CHK(11)) CALL NEWIT1

C

$\mathrm{C}$

$\mathrm{C}$

C

C

C

C

C

C

C

$\mathrm{C}$

C

$\mathrm{C}$$$
\text { C }
$$

IF (NUMS.EQ.CHK(11)) CALL NEWITA

GO TO 260

---IF SOLUTION NOT OBTAINED START NEW ITERATION---

260 IF (TEST.EQ.1.) GO TO 250

C TIME STEPS---

CALL STEADY

---LAST TIME STEP IN PUMPING PERIOD ?---

IF (IFINAL.NE. 1) GO TO 240

---CHECK FOR NEW PUMPING PERIOD---

IF (KPH.EQ.0) GO TO 269

IF (MOD(KP, KPH).EQ. O.AND.PNCH.EQ.CHK(1)) CALL PUNCH

269 IF (KP.LT.NPER) GO TO 230

C ---PUNCHED OUTPUT IF DESIRED---

IF (PNCH.NE.CHK(1)) GO TO 270

IF (KPH.EQ.0) GO TO 271

IF (MOD(KP, KPH) .EQ.0) GO TO 270

271 CALL PUNCH

C ---CHECK FOR NEW PROBLEM---

270 READ $(R, 290, E N D=280)$ NEXT

IF (NEXT.EQ.0) GO TO 10

280 STOP

---CHECK FOR STEADY STATE AND PRINT OUTPUT AT DESIGNATED

290 FORMAT (3I10)

300 FORMAT (' 0 ', $54 \mathrm{X}$, 'WORDS OF Y VECTOR USED $=', \mathrm{I} 7$ )

310 FORMAT (' 0 ' ,62X,' NUMBER OF ROWS $=$ ', I5/60X, 'NUMBER OF COLUMNS $=$ ', I5MAN2660

$1 / 9 \mathrm{X}$, 'NUMBER OF WELLS FOR WHICH DRAWDOWN IS COMPUTED AT A SPECIFIEDMAN2670

2 RADIUS $='$, I5)

MAN2680

320 FORMAT $('-1,36 \mathrm{X}$, 'NO EQUATION SOLVING SCHEME SPECIFIED, EXECUTION TMAN2690

1ERMINATED' $/ 37 \mathrm{X}, 58(\cdot * 1))$

MAN2700

330 FORMAT (' 1 ' ,60X, 'U. S. G. S.' $/ / 55 X$, 'FINITE-DIFFERENCE MODEL' /65X, 'MAN2710

1FOR'/51X, 'SIMULATION OF GROUND-WATER FLOW'//60X, 'JANUARY, 1975'//1MAN2720

$\left.233\left({ }^{\prime} * '\right) /{ }^{\prime} 0^{\prime}, 32 \mathrm{~A} 4 / / 133\left(' *{ }^{\prime}\right)\right)$

340 FORMAT (20A4)

350 FORMAT (A4) 
Table 1.-- Listing of computer program for Laramie County model--Continued

360 FORMAT (' -SIMULATION OPTIONS: ', 13(A4, 4X))

MAN2760

380 FORMAT (G10.0)

MAN2770

END

MAN2780

SUBROUTINE DATAI (PHI , STRT, SURI , T , TR, TC , S , QRE, WELL , TL , SL , PERM, BOTTODAT 10

$1 \mathrm{M}, \mathrm{SY}$, RATE , RIVER , M , TOP , GRND , DELX, DELY, WR, NWR, NODEID , QBND) $\quad$ DAT 20

C
C
$C$
$C$
$C$

READ AND WRITE INPUT DATA

DAT 30

SPECIFICATIONS :

DAT 60

COMMON /SARRAY/ TEST3(102), VF4(11), CHK(15), ITST(102), JTST(102) DAT 80

COMMON /SPARAM/ WATER, CONVRT, EVAP, CHCK, PNCH, NUM, HEAD, CONTR, EROR, LEDAT 90

1 AK , RECH , SIP , U , SS , TT , TMIN , ETDIST , QET , ERR , TMAX , CDLT , HMAX, YDIM, WIDTH , DAT 100

2NUMS , LSOR, ADI , DELT , SUM, SUMP , SUBS , STORE , TE ST , ETQB , ETQD , FACTX, FACTY, DAT 110

3IERR, KOUNT , IF INAL , NUMT , KT , KP , NPER , KTH, ITMAX, LENGTH , NWEL , NW , DIML ,DIDAT 120

4MW, JNO1, INO1 , R , P , PU, I , J , NODE , STDY , KPH, IQPNC DAT 130

COMMON /CK/ ETFLXT, STORT, QRET, CHST, CHDT, FLUXT, PUMPT, CFLUXT, FLXNT DAT 140

COMMON /PR/ XLABEL(3), YLABEL (6), TITLE (5), XN1, MESUR, PRNT (122), BLANKDAT 150

$1(60), \operatorname{DIGIT}(122), \mathrm{VF} 1(6), \mathrm{VF} 2(6), \mathrm{VF} 3(7), \mathrm{XSCALE}, \mathrm{DINCH}, \operatorname{SYM}(17), \mathrm{XN}(100), \mathrm{DAT} 160$

$2 \mathrm{YN}(13), \mathrm{NA}(4), \mathrm{N} 1, \mathrm{~N} 2, \mathrm{~N} 3$, YSCALE , FACT1 , FACT2

COMMON /ARSIZE/ IZ, JZ, IP , JP, IR, JR, IC , JC , IL , JL , IS , JS , IH , IMAX

DAT 170

$\$, I U, J$

COMMON /NDID/ NOD (100), NMBR

DAT 180

COMMON /STREAM/ NRIV,NRPR, NODRV(20), NDR (20), NMRV $(20), Q R I V(3,20)$,

$\$ \operatorname{IRUP}(20), \operatorname{JRUP}(20), \operatorname{IRDN}(20), \operatorname{JRDN}(20)$

DAT 190

DAT 200

DAT 210

DAT 220

C

DIMENSION PHI $(I Z, J Z), \operatorname{STRT}(I Z, J Z)$, SURI $(I Z, J Z), T(I Z, J Z)$, TR(IZ,JZDAT 240

1), $\mathrm{TC}(\mathrm{IZ}, \mathrm{JZ}), \mathrm{S}(\mathrm{IZ}, \mathrm{JZ}), \mathrm{QRE}(\mathrm{IZ}, \mathrm{JZ})$, WELL (IZ,JZ), TL(IZ,JZ), SL(IZ,DAT 250

2JZ), PERM (IP,JP), BOTTOM(IP,JP), SY (IP,JP), RATE (IR, JR), RIVER(IR,DAT 260

3JR), M(IR, JR), TOP(IC,JC), GRND (IL, JL), DELX(JZ), DELY(IZ), WR(IH)DAT 270

$4, \mathrm{NWR}(\mathrm{IH}, 2)$, NODEID (IU, JU) , QBND (IU, JU)

DAT 280

DIMENSION NEXP(18)

DIMENSION NRST (5), $\operatorname{NDST}(5,20), \operatorname{IRUS}(5,20), \operatorname{JRUS}(5,20), \operatorname{IRDS}(5,20)$,

DAT 290

$\$ \operatorname{JRDS}(5,20), \mathrm{QRSR}(5,20)$

C

DIMENSION IWST $(5,100), \operatorname{JWST}(5,100), \operatorname{WLST}(5,100), \operatorname{RADST}(5,100)$

DAT 300

DAT 310

DAT 320

DAT 330

REAL $\star 8 P H I$, DBLE , XLABEL , YLABEL , TITLE , XN 1 , MESUR

DAT 340

REAL *4M

DAT 350

INTEGER R, P, PU, DIML ,DIMW , CHK, WATER , CONVRT , EVAP , CHCK , PNCH, NUM, HEAD , DAT 360

1CONTR , LEAK, RECH , SIP , ADI , STDY

RETURN

DAT 370

DAT 380

$\mathrm{C}$

$\mathrm{C}$

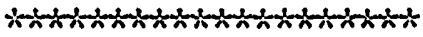

DAT 390

ENTRY DATAIN

DAT 400

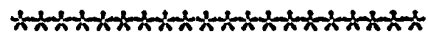

DAT 410

DAT 420

DAT 430

---READ AND WRITE SCALAR PARAMETERS---

DAT 440

READ (R, 810) CONTR, XSCALE, YSCALE, DINCH, FACT1, FACT2, MESUR DAT 450

IF (CONTR.EQ.CHK(3)) WRITE $(P, 1040)$ XSCALE, YSCALE, MESUR, MESUR, DINCDAT 460

$1 \mathrm{H}$, FACT 1 , FACT 2

DAT 470

READ (R, 800) NPER, KTH, ERR, ITMAX, EROR, SS, QET, ETDIST, LENGTH, HMAX, FACDAT 480

1TX, FACTY, KPH

DAT 490

WRITE (P, 840) NPER, KTH, ERR, ITMAX, EROR, SS, QET, ETDIST ,FACTX, FACTY DAT 500 
Table 1.--Listing of computer program for Laramie County model--Continued

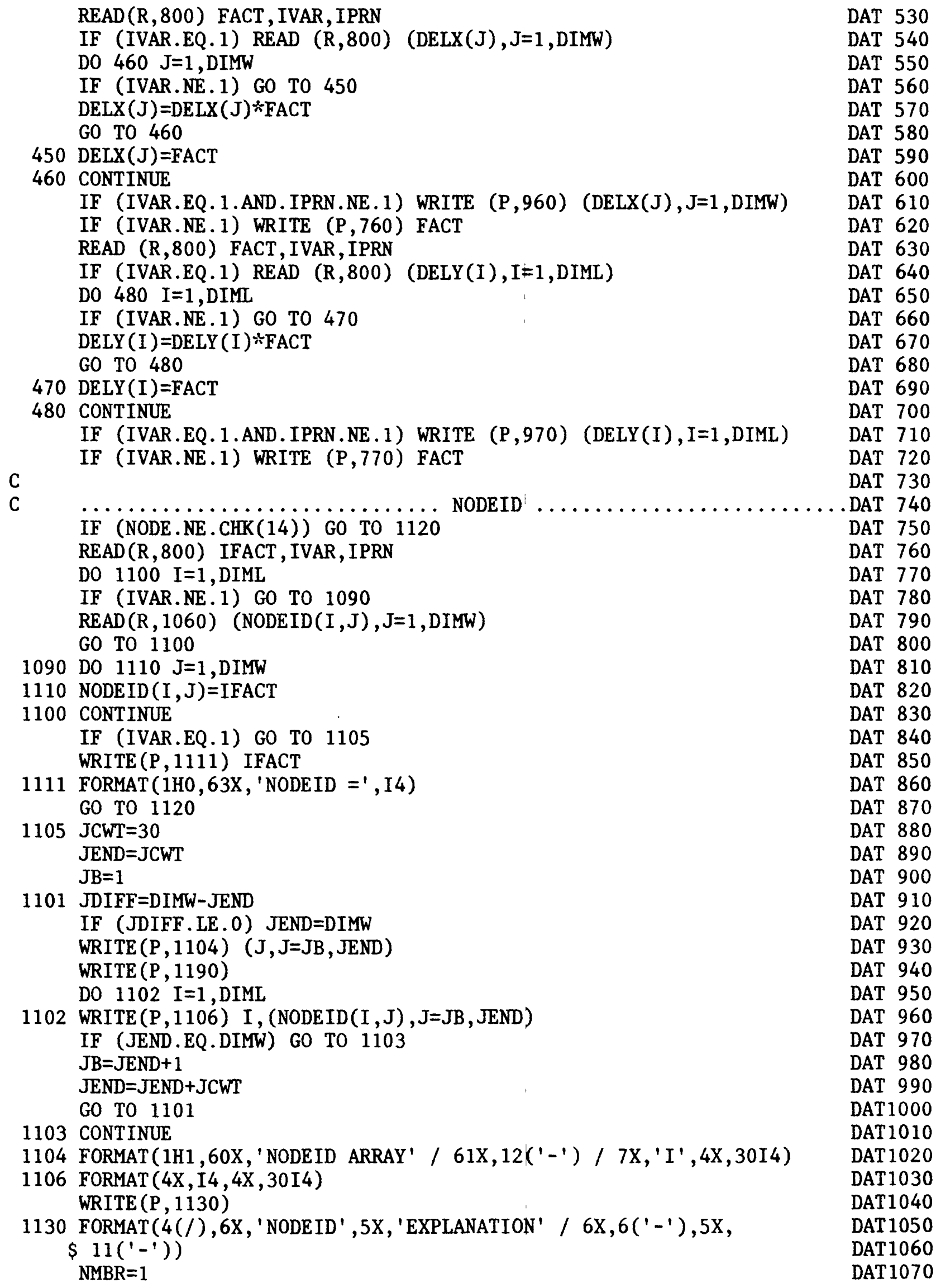


Table 1.--Listing of computer program for Laramie County model--Continued

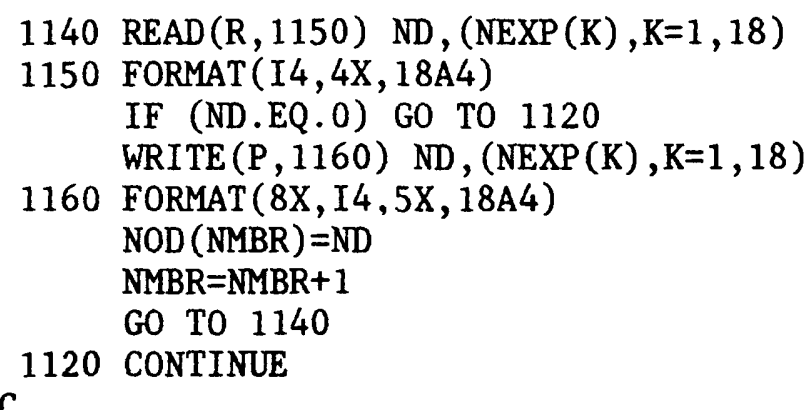

DAT 1080

DAT 1090

DAT 1100

DAT1 110

DAT 1120

DAT 1130

DAT 1140

DAT 1150

C

C ---READ CUMULATIVE MASS BALANCE PARAMETERS---

DAT1160

DAT 1170

READ (R, 1030) SUM, SUMP , PUMPT , CFLUXT , QRET , CHST , CHDT , FLUXT , STORT , ETFDAT 1190

1LXT , FLXNT

DAT 1200

IF (SUM.EQ.0.0) GO TO 20

DAT 1210

WRITE $(P, 790)$ SUM

DAT 1220

$\mathrm{C}$

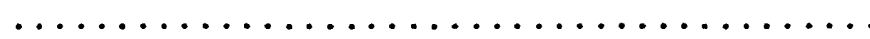

.DAT 1230

C

C -- HEAD DATA TO CONTINUE PREVIOUS COMPUTATIONS READ HERE---

DAT 1240

DO $10 \mathrm{I}=1$, DIML

$\operatorname{READ}(R, 900)(\operatorname{PHI}(I, J), J=1, D I M W)$

DAT 1250

DAT 1260

10 WRITE $(P, 870)$ I, (PHI $(I, J), J=1, D I M W)$

DAT 1270

$\mathrm{C}$

20 READ $(R, 800)$ FACT, IVAR, IPRN

IF (IVAR.EQ. 1.AND.IPRN.EQ.0) WRITE (P,780)

DO 60 I=1, DIML

IF (IVAR.EQ.1) READ $(R, 900)(\operatorname{STRT}(I, J), J=1, D I M W)$

DO $50 \mathrm{~J}=1$, DIMW

IF (IVAR.NE.1) GO TO 30

DAT 1280

$\operatorname{STRT}(I, J)=\operatorname{STRT}(I, J) \div F A C T$

DAT 1290

GO TO 40

$30 \operatorname{STRT}(I, J)=F A C T$

$40 \operatorname{SURI}(I, J)=\operatorname{STRT}(I, J)$

$S(I, J)=0$.

DAT 1300

DAT 1310

DAT 1320

DAT 1330

DAT 1340

DAT 1350

DAT 1360

DAT 1370

DAT 1380

DAT 1390

$\operatorname{TL}(\mathrm{I}, \mathrm{J})=0$.

DAT 1400

$\mathrm{SL}(\mathrm{I}, \mathrm{J})=0$.

DAT 1410

$\mathrm{T}(\mathrm{I}, \mathrm{J})=0$.

DAT 1420

$\operatorname{TR}(\mathrm{I}, \mathrm{J})=0$.

DAT 1430

$\operatorname{TC}(I, J)=0$.

DAT 1440

$\operatorname{WELL}(I, J)=0.0$

DAT 1450

$\mathrm{QRE}(\mathrm{I}, \mathrm{J})=0$.

DAT 1460

IF (LEAK. EQ. CHK (9)) RIVER $(I, J)=0.0$

DAT 1470

IF (LEAK. EQ. CHK (9)) $M(I, J)=0.0$

DAT 1480

IF (CONVRT.EQ.CHK (7)) TOP $(I, J)=0.0$

DAT 1490

DAT 1500

50 IF (SUM.EQ.0.0) PHI $(I, J)=\operatorname{STRT}(I, J)$

DAT 1510

IF (IVAR.EQ. 1.AND.IPRN.EQ.0) WRITE $(P, 870)$ I, (STRT (I,J), J=1,DIMW)

60 CONTINUE

IF (IVAR.NE. 1) WRITE $(P, 640)$ FACT

IF (IVAR.EQ.0.OR.IPRN.NE.2) GO TO 2130

$\mathrm{JCWT}=15$

$\mathrm{JEND}=\mathrm{JCWT}$

DAT 1520

DAT 1530

DAT 1540

DAT1550

DAT 1560

$\mathrm{JB}=1$

2100 JDIFF=DIMW-JEND

IF (JDIFF. LE. 0) JEND=DIMW

DAT 1570

DAT 1580

DAT 1590

WRITE $(P, 2140) \quad(\mathrm{J}, \mathrm{J}=\mathrm{JB}, \mathrm{JEND})$

DAT 1600

WRITE $(P, 1190)$

DAT 1610

DAT 1620 
Table 1.--Listing of computer program for Laramie County model--Continued

DO 2120 I=1, DIML

2120 WRITE $(P, 2150)$ I, (STRT ( I , J ) , J=JB , JEND)

DAT 1630

IF (JEND.EQ.DIMW) GO TO 2130

DAT 1640

$\mathrm{JB}=\mathrm{JEND}+1$

DAT 1650

JEND $=$ JEND + JCWT

DAT 1660

GO TO 2100

DAT 1670

DAT 1680

2130 CONTINUE

DAT 1690

2140 FORMAT(1H1,57X, 'STARTING HEAD ARRAY' / 58X,19('-') / 4X,'I/J',

DAT 1700

$\$ 3 X, 15$ I8)

DAT17 10

2150 FORMAT (I5, 5X, 15F8.1)

DAT 1720

$\mathrm{C}$

S (STORAGE COEFFICIENT)

DAT1730

READ $(R, 800)$ FACT , IVAR, IPRN

IF (IVAR.EQ.1.AND. IPRN.EQ.0) WRITE (P,890)

DAT 1740

DO $90 \mathrm{I}=1$, DIML

IF (IVAR.EQ.1) READ (R,820) ( $(I, J), J=1, D I M W)$

DO $80 \mathrm{~J}=1$, DIMW

IF (IVAR.NE. 1) GO TO 70

$\mathrm{S}(\mathrm{I}, \mathrm{J})=\mathrm{S}(\mathrm{I}, \mathrm{J}) \div \mathrm{FACT}$

DAT 1750

GO TO 80

$70 \mathrm{~S}(\mathrm{I}, \mathrm{J})=\mathrm{FACT}$

80 CONTINUE

90 IF (IVAR.EQ. 1.AND.IPRN.EQ.0) WRITE (P,860) I, (S (I,J),J=1,DIMW)

IF (IVAR.NE. 1) WRITE $(P, 650)$ FACT

IF (IVAR.EQ.0.OR. IPRN.NE.2) GO TO 2230

JCWT $=10$

$\mathrm{JEND}=\mathrm{JCWT}$

$\mathrm{JB}=1$

2200 JDIFF $=$ DIMW - JEND

IF (JDIFF.LE.0) JEND=DIMW

$\operatorname{WRITE}(\mathrm{P}, 2240)(\mathrm{J}, \mathrm{J}=\mathrm{JB}, \mathrm{JEND})$

DAT 1760

WRITE $(\mathrm{P}, 1190)$

DO 2220 I=1, DIML

2220 WRITE $(P, 2250) \mathrm{I},(\mathrm{S}(\mathrm{I}, \mathrm{J}), \mathrm{J}=\mathrm{JB}, \mathrm{JEND})$

IF (JEND.EQ.DIMW) GO TO 2230

$\mathrm{JB}=\mathrm{JEND}+1$

$J E N D=J E N D+J C W T$

DAT 1770

DAT 1780

DAT 1790

DAT 1800

DAT 1810

DAT 1820

DAT 1830

DAT 1840

DAT 1850

DAT 1860

DAT 1870

DAT 1880

DAT 1890

DAT 1900

DAT 1910

DAT 1920

DAT 1930

DAT 1940

DAT 1950

DAT 1960

DAT 1970

DAT 1980

GO TO 2200

DAT 1990

DAT2000

2230 CONTINUE

DAT 2010

2240 FORMAT $(1 \mathrm{Hl}, 54 \mathrm{X}$, 'STORAGE COEFFICIENT ARRAY' / 55X,25('-') /

DAT2020

\$ 4X,' I/J', 3X, 10I12)

DAT2030

2250 FORMAT $(I 5,5 X, 1 \mathrm{P} 10 \mathrm{E} 12.3)$

DAT2040

C

$\mathrm{T}$ (TRANSMISSIVITY)

DAT2050

IF (WATER.EQ.CHK(2)) GO TO 130

DAT2060

READ (R, 800) FACT, IVAR, IPRN

IF (IVAR.EQ. 1.AND.IPRN.EQ.0) WRITE $(P, 850)$

DAT2070

DAT2080

DO $120 \quad I=1$,DIML

DAT2090

IF (IVAR.EQ.1) READ (R, 821) (T (I,J), J=1, DIMW)

DAT2 100

DO $110 \mathrm{~J}=1$, DIMW

DAT 2110

IF (IVAR.NE.1) GO TO 100

DAT2120

$T(I, J)=T(I, J) * F A C T$

GO TO 110

DAT2130

DAT 2140

$100 \mathrm{~T}(\mathrm{I}, \mathrm{J})=\mathrm{FACT}$

DAT2 150

IF (I.EQ.1.OR.I.EQ.DIML.OR.J.EQ.1.OR.J.EQ.DIMW.AND.T(I,J).GE.0.0) DAT2160

$\$ \quad \mathrm{~T}(\mathrm{I}, \mathrm{J})=0.0$

DAT2 170 
Table 1.--Listing of computer program for Laramie County model--Continued

110 CONTINUE

120 IF (IVAR.EQ.1.AND.IPRN.EQ.0) WRITE (P,860) I, (T ( I, J), J=1, DIMW)

IF (IVAR.NE. 1) WRITE (P,700) FACT

IF (IVAR.EQ.0.OR.IPRN.NE.2) GO TO 2330

JCWT $=10$

JEND $=\mathrm{JCWT}$

$\mathrm{JB}=1$

2300 JDIFF=DIMW-JEND

IF (JDIFF .LE. 0) JEND=DIMW

WRITE $(P, 2340) \quad(J, J=J B, J E N D)$

WRITE $(P, 1190)$

DO $2320 \quad \mathrm{I}=1$, DIML

2320 WRITE $(P, 2250)$ I , ( $(I, J), J=J B, J E N D)$

IF (JEND.EQ.DIMW) GO TO 2330

$\mathrm{JB}=\mathrm{JEND}+1$

JEND= JEND+JCWT

GO TO 2300

2330 CONTINUE

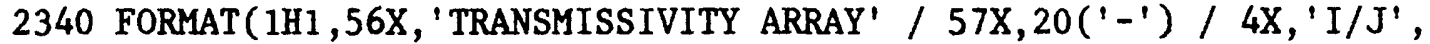

$\$ 3 \mathrm{X}, 10 \mathrm{I} 12)$

GO TO 260

DAT2 180

DAT2 190

DAT2200

DAT2210

DAT2220

DAT2230

DAT2240

DAT2250

DAT2260

DAT2270

DAT2280

DAT2290

DAT2300

DAT2310

DAT2320

DAT2330

DAT2340

DAT2350

DAT2360

DAT2370

DAT 2380

DAT2390

C

130 READ $(R, 800)$ FACT, IVAR, IPRN, ICON, IPRT

IF (ICON.EQ. 1.AND. IPRT.NE.1) GO TO 136

WRITE $(P, 1065) \quad(J, J=1, D I M W)$

DO $134 \mathrm{I}=1$, DIMI

IF (IVAR.EQ.1) $\operatorname{READ}(R, 822)(\operatorname{PERM}(I, J), J=1, D I M W)$

DO $132 \mathrm{~J}=1$, DIMW

IF (IVAR.NE.1) GO TO 136

$\operatorname{IPERM}(J)=(\operatorname{PERM}(I, J)+0.5) * 0.1$

IF $(\operatorname{PERM}(\mathrm{I}, \mathrm{J}) . \operatorname{LE} .9 .0) \operatorname{IPERM}(\mathrm{J})=(\operatorname{PERM}(I, J)+0.5)$

132 CONTINUE

IF (IVAR.EQ.1.AND.IPRT.NE. 1) WRITE (P, 1070) I, (IPERM(J), J=1,DIMW), II

134 CONTINUE

WRITE $(P, 1080)(J, J=1, D I M W)$

136 CONTINUE

IF (IVAR.EQ. 1.AND. IPRN.EQ.0) WRITE $(P, 910)$

DO $160 \mathrm{I}=1$, DIML

DO $150 \mathrm{~J}=1$, DIMW

IF (IVAR.NE.1) GO TO 140

$T(I, J)=\operatorname{PERM}(I, J)$

$\operatorname{PERM}(\mathrm{I}, \mathrm{J})=\operatorname{PERM}(\mathrm{I}, \mathrm{J}) * \mathrm{FACT}$

GO TO 150

140 PERM $(I, J)=F A C T$

IF (I.EQ.1.OR. I.EQ.DIML.OR.J.EQ.1.OR.J.EQ.DIMW.AND.PERM(I, J) .GE.

$\$ \quad 0.0) \operatorname{PERM}(I, J)=0.0$

150 CONTINUE

160 IF (IVAR.EQ.1.AND.IPRN.EQ.0) WRITE (P, 860) I, (PERM(I,J), J=1, DIMW)

IF (IVAR.NE. 1) WRITE (P,710) FACT

IF (IPRN.NE.2) GO TO 164

$\mathrm{JCWT}=21$

JEND $=J C W T$

$\mathrm{JB}=1$
.DAT2400

DAT2410

DAT2420

DAT2430

DAT2440

DAT2 450

DAT2 460

DAT2 470

DAT 2480

DAT2 490

DAT2500

, DAT 2510

DAT2520

DAT2530

DAT2540

DAT2550

DAT2560

DAT 2570

DAT2580

DAT2590

DAT2600

DAT2610

DAT2620

DAT2630

DAT2640

DAT2650

DAT 2660

DAT2670

DAT 2680

DAT2690

DAT 2700

DAT2710

DAT2720 
Table 1.--Listing of computer program for Laramie County model--Continued

161 JDIFF=DIMW-JEND

IF (JDIFF.LE.0) JEND=DIMW

DAT2730

WRITE $(P, 6160)(\mathrm{J}, \mathrm{J}=\mathrm{JB}, \mathrm{JEND})$

DAT2740

DO 162 I=1, DIML

$162 \operatorname{WRITE}(P, 6161)$ I, (T ( I , J ) , J=JB, JEND)

DAT 2750

DAT2760

IF (JEND.EQ.DIMW) GO TO 163

DAT 2770

$\mathrm{JB}=\mathrm{JEND}+1$

DAT2780

JEND $=J E N D+J C W T$

DAT2790

GO TO 161

DAT2800

DAT2810

DAT2820

163 WRITE $(P, 6162)$ FACT

DAT 2830

$\$ 6 \mathrm{X}, 2116)$

DAT2840

6161 FORMAT (1H0, I4, 1X, 21F6.2)

DAT2850

6162 FORMAT (1HO, 5X, 'HYDRAULIC CONDUCTIVITY MULTIPLICATION FACTOR =',

DAT2860 $\$$ IPE 12.4)

DAT2870

164 CONTINUE

DAT2880

C

READ $(R, 800)$ FACT, IVAR, IPRN

BOTTOM (AQ. BOTTOM EL.) ........DAT2890

IF (IVAR.EQ.1.AND.IPRN.EQ.0) WRITE (P,920)

DAT2900

DO 190 I=1,DIML

DAT2910

IF (IVAR.EQ. 1) READ (R, 822) (BOTTOM(I,J), J=1,DIMW)

DAT2920

DO $180 \mathrm{~J}=1$, DIMW

DAT 2930

IF (IVAR.NE.1) GO TO 170

DAT2940

BOTTOM $(I, J)=$ BOTTOM $(I, J) * F A C T$

DAT2950

GO TO 180

DAT2960

DAT2970

$170 \operatorname{BOTTOM}(I, J)=$ FACT

DAT2980

180 CONTINUE

DAT2990

190 IF (IVAR.EQ.1.AND. IPRN.EQ.0) WRITE (P,870) I, (BOTTOM(I,J) ,J=1, DIMWDAT3000

1)

IF (IVAR.NE.1) WRITE (P,720) FACT

IF (IVAR.EQ.0.OR. IPRN.NE.2) GO TO 2430

JCWT $=15$

JEND $=\mathrm{JCWT}$

$\mathrm{JB}=1$

2400 JDIFF=DIMW-JEND

IF (JDIFF.LE.0) JEND=DIMW

DAT3010

DAT3020

DAT 3030

DAT 3040

DAT3050

DAT 3060

$\operatorname{WRITE}(P, 2440) \quad(\mathrm{J}, \mathrm{J}=\mathrm{JB}, \mathrm{JEND})$

DAT3070

WRITE $(P, 1190)$

DAT3080

DAT3090

DO $2420 \quad \mathrm{I}=1$, DIML

DAT 3100

DAT3110

2420 WRITE $(P, 2150)$ I, (BOTTOM $(I, J), J=J B, J E N D)$

DAT3120

IF (JEND.EQ.DIMW) GO TO 2430

DAT3130

$\mathrm{JB}=\mathrm{JEND}+1$

DAT3140

JEND=JEND+JCWT

DAT3150

GO TO 2400

DAT3160

2430 CONTINUE

DAT3170

DAT3180

DAT3190

$\left.\$ 45 \mathrm{X}, 40\left(^{\prime}-^{\prime}\right) / 4 \mathrm{X},{ }^{\prime} \mathrm{I} / \mathrm{J}^{\prime}, 3 \mathrm{X}, 15 \mathrm{I}\right)$

DAT3200

DAT3210

C --CALCULATE AND PRINT INITIAL SATURATED THICKNESS AND

DAT 3220 TRANSMISSIVITY ARRAYS

DAT3230

DO 1200 I=1,DIML

DO $1200 \mathrm{~J}=1$, DIMW

DAT3240

$1200 \mathrm{~T}(\mathrm{I}, \mathrm{J})=\operatorname{STRT}(\mathrm{I}, \mathrm{J})-\operatorname{BOTTOM}(\mathrm{I}, \mathrm{J})$

DAT3250

JCWT $=15$

DAT 3260

$\mathrm{JEND}=\mathrm{JCWT}$

DAT3270 
Table 1.--Listing of computer program for Laramie County mode1--Continued

$\mathrm{JB}=1$

$1210 \mathrm{JDIFF}=\mathrm{DIMW}-\mathrm{JEND}$

IF (JDIFF.LE. O) JEND=DIMW

WRITE $(P, 1290)(\mathrm{J}, \mathrm{J}=\mathrm{JB}, \mathrm{JEND})$

WRITE $(\mathrm{P}, 1190)$

DO $1220 \mathrm{I}=1, \mathrm{DIML}$

1220 WRITE $(P, 2850) \mathrm{I},(\mathrm{T}(\mathrm{I}, \mathrm{J}), \mathrm{J}=\mathrm{JB}, \mathrm{JEND})$

IF (JEND.EQ.DIMW) GO TO 1230

$\mathrm{JB}=\mathrm{JEND}+1$

$J E N D=J E N D+J C W T$

GO TO 1210

1230 DO 1240 I=1,DIML

DO $1240 \mathrm{~J}=1$, DIMW

$1240 \mathrm{~T}(\mathrm{I}, \mathrm{J})=\mathrm{T}(\mathrm{I}, \mathrm{J}) \div \operatorname{PERM}(\mathrm{I}, \mathrm{J})$

$\mathrm{JCWT}=10$

$\mathrm{JEND}=\mathrm{JCWT}$

DAT 3280

DAT3290

DAT3300

DAT3310

DAT3320

DAT3330

DAT3340

DAT3350

DAT3360

DAT3370

DAT 3380

DAT3390

DAT 3400

DAT3410

DAT3420

$\mathrm{JB}=1$

DAT3430

DAT3440

1250 JDIFF=DIMW-JEND

IF (JDIFF.LE.0) JEND=DIMW

DAT3450

WRITE $(P, 2340) \quad(J, J=J B, J E N D)$

DAT3460

WRITE $(P, 1190)$

DO $1260 \quad I=1$, DIML

DAT3470

DAT 3480

DAT3490

1260 WRITE $(P, 2250)$ I , (T $(I, J), J=J B, J E N D)$

DAT3500

IF (JEND.EQ.DIMW) GO TO 1270

DAT35 10

$\mathrm{JB}=\mathrm{JEND}+1$

$J E N D=J E N D+J C W T$

DAT3520

GO TO 1250

DAT3530

DAT 3540

1270 CONTINUE

1290 FORMAT $(1 \mathrm{H} 1,53 \mathrm{X}$, 'INITIAL SATURATED THICKNESS' / 54X,27('-') /

DAT3550

$\left.\$ 4 X, ' I / J^{\prime}, 3 X, 15 I 8\right)$

DAT3560

DAT3570

C

DAT 3580

C

SY (SPECIFIC YIELD)

DAT3590

READ (R, 800) FACT, IVAR, IPRN

DAT3600

IF (IVAR.EQ. 1.AND.IPRN.EQ.0) WRITE (P, 1050)

DAT 3610

DO 220 I=1, DIML

IF (IVAR.EQ.1) READ (R, 820) (SY $(I, J), J=1, D I M W)$

DO $210 \mathrm{~J}=1$, DIMW

IF (IVAR.NE. 1) GO TO 200

DAT3620

DAT 3630

DAT 3640

$\operatorname{SY}(I, J)=S Y(I, J) * F A C T$

DAT3650

GO TO 210

DAT 3660

DAT3670

$200 \mathrm{SY}(\mathrm{I}, \mathrm{J})=\mathrm{FACT}$

DAT3680

210 CONTINUE

DAT3690

220 IF (IVAR.EQ. 1.AND. IPRN.EQ. 0) WRITE (P, 880) I, (SY ( I , J) , J=1, DIMW)

DAT 3700

IF (IVAR.NE. 1) WRITE $(P, 660)$ FACT

DAT3710

IF (IVAR.EQ.0.OR. IPRN.NE.2) GO TO 2530

DAT3720

JCWT $=15$

DAT 3730

JEND $=$ JCWT

DAT3740

$\mathrm{JB}=1$

DAT 3750

DAT3760

2500 JDIFF=DIMW - JEND

IF (JDIFF.LE.0) JEND=DIMW

DAT 3770

WRITE $(P, 2540) \quad(J, J=J B, J E N D)$

DAT 3780

WRITE $(P, 1190)$

DAT3790

DO $2520 \quad \mathrm{I}=1$, DIML

DAT 3800

2520 WRITE $(P, 2850)$ I , (SY ( I , J ) , J=JB, JEND)

DAT 3810

IF (JEND.EQ.DIMW) GO TO 2530 
Table 1.--Listing of computer program for Laramie County model--Continued

$\mathrm{JB}=\mathrm{JEND}+1$

$\mathrm{JEND}=\mathrm{JEND}+\mathrm{JCWT}$

DAT3830

GO TO 2500

DAT3840

DAT3850

2530 CONTINUE

DAT3860

2540 FORMAT $(1 \mathrm{H} 1,56 \mathrm{X}$, 'SPECIFIC YIELD ARRAY' / 57X,20('-') / 4X,' I/J', $\$ 3 X, 15 I 8)$

DAT3870

DAT3880

DAT3890

C

M (CONF. BED THICKNESS)

DAT3900

IF (LEAK.NE.CHK (9)) GO TO 260

DAT3910

READ (R, 800) FACT, IVAR, IPRN, NRVR

DAT3920

IF (NRVR.EQ. O) GO TO 322

DAT3930

DO $321 \mathrm{NR}=1$, NRVR

DAT3940

$321 \operatorname{READ}(\mathrm{R}, 800) \mathrm{I}, \mathrm{J}, \mathrm{M}(\mathrm{I}, \mathrm{J})$

322 CONTINUE

IF (IVAR.EQ. 1.AND.IPRN.EQ.0) WRITE (P, 1020)

DAT3950

DO $350 \quad I=1$, DIML

IF (IVAR.EQ.1) READ(R,900) (M(I,J),J=1,DIMW)

DAT3960

DAT3970

DAT3980

D0 $340 \mathrm{~J}=1$, DIMW

DAT3990

IF (IVAR.NE. 1) GO TO 330

DAT 4000

$\mathrm{M}(\mathrm{I}, \mathrm{J})=\mathrm{M}(\mathrm{I}, \mathrm{J}) \div \mathrm{FACT}$

DAT4010

GO TO 340

330 IF $(M(I, J) . E Q .0 .0) \quad M(I, J)=F A C T$

340 CONTINUE

350 IF (IVAR.EQ. 1.AND. IPRN.EQ.0) WRITE (P, 860) I, (M(I,J), J=1,DIMW)

IF (IVAR.EQ. 0.AND.IPRN.NE.2) WRITE $(P, 690)$ FACT

IF (IPRN.NE.2) GO TO 2830

$\mathrm{JCWT}=15$

JEND $=\mathrm{JCWT}$

$\mathrm{JB}=1$

2800 JDIFF $=$ DIMW - JEND

IF (JDIFF.LE.0) JEND=DIMW

WRITE $(P, 2840) \quad(J, J=J B, J E N D)$

WRITE $(P, 1190)$

DO 2820 I=1,DIML

2820 WRITE $(P, 2850) I,(M(I, J), J=J B, J E N D)$

DAT4020

DAT4030

DAT 4040

DAT4050

DAT4060

DAT 4070

DAT4080

DAT4090

DAT4100

DAT 4110

DAT4 120

DAT4130

DAT 4140

DAT 4150

DAT 4160

IF (JEND.EQ.DIMW) GO TO 2830

DAT4170

DAT4180

$\mathrm{JB}=\mathrm{JEND}+1$

JEND $=$ JEND + JCWT

DAT4190

GO TO 2800

DAT4210

2830 CONT INUE

DAT 4220

DAT4230

DAT4240

$\$$ ' $\left.I / J^{\prime}, 3 X, 15 I 8\right)$

DAT4250

2850 FORMAT $(I 5,5 X, 15 F 8.2)$

DAT4260

$\mathrm{C}$

RATE (CONF. BED CONDUCTIVITY)

DAT4270

READ (R, 800) FACT, IVAR, IPRN, IRATE , HDIFF, TDIFF, CRATE

DAT4280

IF (IVAR.EQ. 1.AND. IPRN.EQ.0) WRITE $(P, 830)$

DAT 4290

DO 290 I=1, DIML

DAT 4300

IF (IVAR.EQ. 1) READ $(R, 821)$ (RATE $(I, J), J=1, D I M W)$

DO $280 \mathrm{~J}=1$, DIMW

DAT 4310

IF (IVAR.NE. 1) GO TO 270

DAT 4320

$\operatorname{RATE}(I, J)=\operatorname{RATE}(I, J) \div F A C T$

DAT 4330

GO TO 279

DAT4340

DAT 4350

$270 \operatorname{RATE}(\mathrm{I}, \mathrm{J})=\mathrm{FACT}$

279 IF (IRATE.EQ.0) GO TO 280 
Table 1.--Listing of computer program for Laramie County model--Continued

IF (HDIFF.EQ.0.0) HDIFF $=1.0$

DAT 4380

IF (TDIFF.EQ.0.0) TDIFF $=1.0$

DAT 4390

280 CONTINUE

DAT 4400

290 IF (IVAR.EQ.1.AND.IPRN.EQ.0) WRITE (P, 860) I, (RATE $(I, J), J=1, D I M W)$ DAT4410

IF (IVAR.NE. 1) WRITE $(P, 670)$ FACT

IF (IVAR.EQ.0.OR.IPRN.NE.2) GO TO 2630

DAT 4420

JCWT $=10$

JEND $=\mathrm{JCWT}$

$\mathrm{JB}=1$

2600 JDIFF $=D I M W-J E N D$

IF (JDIFF.LE.0) JEND=DIMW

DAT 4430

DAT 4440

DAT 4450

DAT 4460

DAT 4470

DAT 4480

WRITE $(P, 2640) \quad(J, J=J B, J E N D)$

DAT4490

WRITE $(P, 1190)$

DAT 4500

DO $2620 \quad \mathrm{I}=1$, DIML

DAT 4510

2620 WRITE $(P, 2250)$ I , (RATE $(I, J), J=J B, J E N D)$

DAT4520

IF (JEND.EQ.DIMW) GO TO 2630

DAT 4530

$\mathrm{JB}=\mathrm{JEND}+1$

JEND $=\mathrm{JEND}+\mathrm{JCWT}$

DAT 4540

GO TO 2600

DAT 4550

DAT 4560

2630 CONTINUE

DAT 4570

2640 FORMAT ( $1 \mathrm{H} 1,44 \mathrm{X}$, 'HYDRAULIC CONDUCTIVITY OF THE CONFINING BED' /

DAT 4580

$\left.\$ 45 \mathrm{X}, 43\left('-{ }^{\prime}\right) / 4 \mathrm{X},{ }^{\prime} \mathrm{I} / \mathrm{J}^{\prime}, 3 \mathrm{X}, 10 \mathrm{I} 12\right)$

$\mathrm{C}$

C

READ (R, 800) FACT, IVAR, IPRN, NRVR

IF (NRVR.EQ.0) GO TO 292

DO $291 \mathrm{NR}=1$, NRVR

$291 \operatorname{READ}(\mathrm{R}, 800) \mathrm{I}, \mathrm{J}, \mathrm{RIVER}(\mathrm{I}, \mathrm{J})$

292 IF (IVAR.EQ. 1.AND. IPRN.EQ.0) WRITE (P, 980)

DO 320 I $=1$, DIML

IF (IVAR.EQ. 1) READ(R,900) (RIVER $(I, J), J=1, D I M W)$

DO $310 \mathrm{~J}=1$, DIMW

IF (IVAR.NE. 1) GO TO 300

$\operatorname{RIVER}(I, J)=\operatorname{RIVER}(I, J) * F A C T$

GO TO 309

$300 \operatorname{IF}(\operatorname{RIVER}(I, J) . E Q .0 .0) \operatorname{RIVER}(I, J)=F A C T$

309 IF (IRATE.EQ.0) GO TO 310

IF $(M(I, J)$.NE.0.0.AND.RIVER(I,J).EQ.0.0) $\operatorname{RIVER}(I, J)=\operatorname{STRT}(I, J)-$

$\$ M(I, J) * \operatorname{RATE}(I, J) /(\operatorname{CRATE} * \operatorname{DELX}(J) * \operatorname{DELY}(\mathrm{I}))$

$\operatorname{RATE}(\mathrm{I}, \mathrm{J})=$ CRATE

310 CONTINUE

320 IF (IVAR.EQ.1.AND.IPRN.EQ.0) WRITE (P, 870) I, (RIVER(I,J), J=1, DIMW)

IF (IVAR.EQ.0.AND.IPRN.NE.2) WRITE $(P, 680)$ FACT

IF (IPRN.NE.2) GO TO 2730

JCWT $=15$

JEND $=$ JCWT

$\mathrm{JB}=1$

2700 JDIFF $=$ DIMW-JEND

IF (JDIFF.LE.0) JEND=DIMW

WRITE $(P, 2740) \quad(J, J=J B, J E N D)$

WRITE $(P, 1190)$

DO $2720 \quad I=1$, DIML

2720 WRITE $(P, 2150)$ I , (RIVER $(I, J), J=J B, J E N D)$

IF (JEND.EQ.DIMW) GO TO 2730

$\mathrm{JB}=\mathrm{JEND}+1$
DAT4590

DAT4600

DAT4610

DAT4620

DAT 4630

DAT4640

DAT 4650

DAT4660

DAT4670

DAT4680

DAT4690

DAT4700

DAT 4710

DAT 4720

DAT4730

DAT 4740

DAT4750

DAT 4760

DAT 4770

DAT 4780

DAT 4790

DAT 4800

DAT4810

DAT4820

DAT4830

DAT4840

DAT4850

DAT4860

DAT4870

DAT4880

DAT4890

DAT4900

DAT4910

DAT4920 
Table 1.--Listing of computer program for Laramie County model--Continued

JEND $=\mathrm{JEND}+\mathrm{JCWT}$

DAT 4930

GO TO 2700

DAT 4940

2730 CONTINUE

2740 FORMAT(1H1,58X, 'RIVER HEAD ARRAY' / 59X, 16('-') / 4X, 'I/J',

DAT 4950

$\$ 3 \mathrm{X}, 15 \mathrm{I} 8$ )

DAT4960

C

C

260 IF (CONVRT.NE.CHK(7)) GO TO 358

TOP (AQ. TOP ELEVATION)

DAT4970

DAT4980

READ $(R, 800)$ FACT, IVAR, IPRN, NRVR

DAT4990

IF (NRVR.EQ.0) GO TO 262

DO 261 NR=1, NRVR

$261 \operatorname{READ}(R, 800) \mathrm{I}, \mathrm{J}, \operatorname{TOP}(\mathrm{I}, \mathrm{J})$

262 CONTINUE

IF (IVAR.EQ.1.AND.IPRN.EQ.0) WRITE $(P, 930)$

DAT5000

DAT5010

DAT5020

DAT5030

DO 250 I=1,DIML

IF (IVAR.EQ.1) $\operatorname{READ}(\mathrm{R}, 900)(\operatorname{TOP}(I, J), J=1, \mathrm{DIMW})$

DO $240 \mathrm{~J}=1$, DIMW

IF (IVAR.NE.1) GO TO 230

DAT5040

$\operatorname{TOP}(I, J)=\operatorname{TOP}(I, J) * F A C T$

GO TO 240

230 IF $(T O P(I, J) . E Q \cdot 0.0)$ TOP $(I, J)=F A C T$

240 IF $(M(I, J) \cdot N E \cdot 0 \cdot 0 . \operatorname{AND} \cdot \operatorname{TOP}(I, J) \cdot E Q \cdot 0.0) \operatorname{TOP}(I, J)=R I V E R(I, J)-$ $\$$ TDIFF

250 IF (IVAR.EQ.1.AND. IPRN.EQ.0) WRITE (P, 870) $I,(\operatorname{TOP}(I, J), J=1, D I M W)$

DAT5050

DAT5060

DAT5070

DAT5080

DAT5090

DAT5 100

DAT5 110

DAT5 120

DAT5 130

DAT5 140

DAT5 150

DAT5 160

IF (IVAR.EQ.0.AND.IPRN.NE.2) WRITE (P,730) FACT

IF (IPRN.NE.2) GO TO 2930

DAT5 170

DAT5 180

DAT5 190

$\mathrm{JCWT}=15$

JEND $=J C W T$

DAT5200

$\mathrm{JB}=1$

2900 JDIFF=DIMW-JEND

IF (JDIFF.LE.0) JEND=DIMW

WRITE $(P, 2940) \quad(J, J=J B, J E N D)$

WRITE $(P, 1190)$

DO 2920 I=1, DIMI

$2920 \operatorname{WRITE}(\mathrm{P}, 2150)$ I , (TOP (I, J ) , J=JB, JEND)

DAT5210

DAT5220

DAT5230

DAT5240

DAT5250

DAT5260

IF (JEND.EQ.DIMW) GO TO 2930

DAT5270

$\mathrm{JB}=\mathrm{JEND}+1$

DAT5280

JEND $=$ JEND + JCWT

DAT5290

GO TO 2900

DAT5300

DAT5310

2930 CONTINUE

2940 FORMAT (1H1,58X, 'AQUIFER TOP ARRAY' / 59X,17('-') / 4X,'I/J',

$\$ 3 X, 1518)$

358 IF (LEAK.NE.CHK(9)) GO TO 360

DO 359 I=1, DIML

DO $359 \mathrm{~J}=1$, DIMW

DAT5320

DAT5330

DAT5340

DAT5 350

DAT5360

DAT5370

$359 \operatorname{RATE}(I, J)=\operatorname{ABS}(\operatorname{RATE}(I, J))$

DAT5380

DAT5390

c

GRND (LAND ELEVATION)

DAT5 400

360 IF (EVAP.NE.CHK(6)) GO TO 400

DAT5 410

READ $(R, 800)$ FACT, IVAR, IPRN

DAT5420

IF (IVAR.EQ.1.AND.IPRN.EQ.0) WRITE ( $P, 940)$

DAT5 430

DO $390 \quad I=1$, DIML

IF (IVAR.EQ . 1) READ $(R, 900)(\operatorname{GRND}(I, J), J=1, D I M W)$

DAT5440

DO $380 \mathrm{~J}=1$, DIMW

DAT5 450

IF (IVAR.NE. 1) GO TO 370 
Table 1.--Listing of computer program for Laramie County model--Continued

$\operatorname{GRND}(\mathrm{I}, \mathrm{J})=\operatorname{GRND}(\mathrm{I}, \mathrm{J}) * \mathrm{FACT}$

DAT5480

GO TO 380

DAT5490

$370 \operatorname{GRND}(I, J)=F A C T$

DAT5500

380 CONTINUE

DAT5510

390 IF (IVAR.EQ.1.AND.IPRN.EQ.0) WRITE $(P, 870) I,(\operatorname{GRND}(I, J), J=1, D I M W)$

IF (IVAR.NE. 1) WRITE (P,740) FACT

IF (IVAR.EQ.0.OR. IPRN.NE.2) GO TO 3030

DAT5520

JCWT $=15$

JEND $=J C W T$

$\mathrm{JB}=1$

3000 JDIFF $=$ DIMW - JEND

IF (JDIFF.LE.0) JEND=DIMW

DAT5530

WRITE $(P, 3040) \quad(J, J=J B, J E N D)$

DAT5540

DAT5550

DAT5560

DAT5570

WRITE $(P, 1190)$

DO $3020 \quad I=1$, DIML

DAT5580

DAT5590

DAT5600

DAT5610

DAT5620

3020 WRITE $(P, 2150)$ I, (GRND ( I , J ) , J=JB , JEND)

DAT5630

IF (JEND.EQ.DIMW) GO TO 3030

DAT5640

$J B=J E N D+1$

DAT5650

JEND = JEND+JCWT

DAT5660

GO TO 3000

DAT5670

3030 CONTINUE

DAT5 680

3040 FORMAT (1H1,55X, 'LAND SURFACE ELEVATION' / 56X,22('-') / 4X,

DAT5690

$\$ ' I / J ', 3 X, 15 I 8)$

DAT5 700

C

C

400 IF (RECH.NE.CHK(10)) GO TO 440

QRE (RECHARGE RATE)

DAT5710

DAT5720

READ $(R, 800)$ FACT, IVAR, IPRN

DAT5730

IF (IVAR.EQ.1.AND.IPRN.EQ.0) WRITE $(P, 950)$

DAT5740

DO $430 \quad I=1$, DIML

IF (IVAR.EQ. 1) READ (R, 820) (QRE (I, J), J=1, DIMW)

DO $420 \mathrm{~J}=1$, DIMW

IF (IVAR.NE.1) GO TO 410

DAT5750

DAT5760

DAT5770

$\mathrm{QRE}(I, J)=Q R E(I, J) * F A C T$

DAT5780

GO TO 420

$410 \mathrm{QRE}(I, J)=F A C T$

420 CONTINUE

430 IF (IVAR.EQ.1.AND.IPRN.EQ.0) WRITE (P,860) I, (QRE (I,J),J=1, DIMW)

IF (IVAR.NE . 1) WRITE (P,750) FACT

IF (IVAR.EQ.0.OR.IPRN.NE.2) GO TO 3130

JCWT $=10$

JEND $=$ JCWT

$\mathrm{JB}=1$

3100 JDIFF $=$ DIMW-JEND

IF (JDIFF.LE.0) JEND=DIMW

DAT5790

DAT5800

DAT5810

DAT5820

DAT5830

DAT5840

DAT5850

DAT5 860

DAT5870

DAT5880

DAT5890

DAT5900

DAT5 910

WRITE $(P, 3140) \quad(J, J=J B, J E N D)$

DAT5920

WRITE $(P, 1190)$

DO $3120 \quad I=1$, DIML

DAT5930

DAT5940

DAT5950

DAT5 960

IF (JEND.EQ.DIMW) GO TO 3130

$\mathrm{JB}=\mathrm{JEND}+1$

DAT5970

JEND $=$ JEND $+J C W T$

DAT5 980

GO TO 3100

DAT5990

3130 CONTINUE

DAT6000

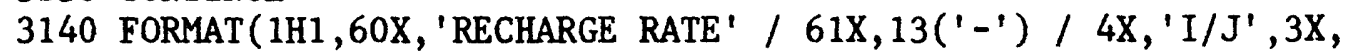

DAT6010

$\$ 10 \mathrm{I} 12$ )

DAT6020 
Table 1.--Listing of computer program for Laramie County model--Continued

440 CONTINUE

DAT6030

$\mathrm{C}$

C ---READ IN VALUES OF NODEID $(\mathrm{I}, \mathrm{J})$ TO BE ASSOCIATED WITH LEAKY

DAT6040

C STREAM NODES---

NRIV $=0$

NRPR $=0$

C IF (LEAK.NE.CHK(9)) GO TO 489

IF (NODE.NE.CHK(14)) GO TO 489

IF (CONVRT.NE.CHK(7)) GO TO 489

$\operatorname{READ}(R, 800)$ NRIV

C

IF (NRIV.NE.0) READ(R, 1060) (NODRV(I), I=1, NRIV)

489 CONTINUE

C ---INITIALIZE VARIABLES---

DO 503 II $=1,5$

$\operatorname{NRST}($ II $)=0$

DO 501 IS $=1,20$

$\operatorname{NDST}($ II , IS $)=0$

$\operatorname{IRUS}(I I, I S)=0$

$\operatorname{JRUS}(I I, I S)=0$

$\operatorname{IRDS}(I I, I S)=0$

$\operatorname{JRDS}(I I, I S)=0$

DAT6050

DAT6060

DAT6070

DAT6080

DAT6090

DAT6100

DAT6110

DAT6120

DAT6130

DAT6140

DAT6150

DAT6160

DAT6170

DAT6180

DAT6190

DAT6200

DAT6210

DAT6220

DAT6230

DAT6240

501 QRSR(II, IS $)=0.0$

DAT6250

DO $502 \mathrm{IW}=1,100$

DAT6260

$\operatorname{IWST}(I I, I W)=0$

DAT6270

JWST $(I I, I W)=0$

DAT6280

WLST $(I I, I W)=0.0$

DAT6290

$502 \operatorname{RADST}(I I, I W)=0.0$

DAT6300

503 CONTINUE

DAT6310

JN01 =DIMW -1

DAT6320

INO1=DIML - 1

DAT6330

IF (LEAK.NE.CHK(9).OR.SS.NE.0.) GO TO 500

DAT6340

DO $490 \mathrm{I}=2$, INO1

DAT6350

DO $490 \mathrm{~J}=2$, JNO1

DAT6360

IF (M(I,J).EQ.0.) GO TO 490

DAT6370

$\operatorname{TL}(I, J)=\operatorname{RATE}(I, J) / M(I, J)$

DAT6380

490 CONTINUE

$500 \mathrm{ETQB}=0.0$

$\mathrm{ETQD}=0.0$

SUBS $=0.0$

DAT6390

DAT6400

DAT6410

$\mathrm{U}=1.0$

$\mathrm{TT}=0.0$

DAT6420

DAT6430

DAT6440

IM $=$ MINO $(6 * \mathrm{DIMW}+4,124)$

DAT6450

IM $=(132-I M) / 2$

WIDTH $=0$.

DO $510 \mathrm{~J}=2$, JNO1

DAT6460

DAT6470

DAT6480

510 WIDTH $=$ WIDTH + DELX (J)

DAT6490

YDIM $=0$.

DAT6500

DO $520 \mathrm{I}=2$, INO1

DAT6510

520 YDIM=YDIM+DELY (I)

DAT6520

DAT6530

C

---READ IN OR CALCULATE RECHARGE/DISCHARGE RATES AT CONSTANT FLUX

DAT6540

C BOUNDARY NODES: THESE NODES ARE FLAGGED BY NEGATIVE VALUES

DAT6550 OF NODEID $(\mathrm{I}, \mathrm{J})---$

IF (NODE.NE.CHK(14)) GO TO 525

DAT6560

DAT6570 
Table 1.--Listing of computer program for Laramie County model--Continued

$\operatorname{READ}(R, 800)$ FACT , IVAR, IPRN, IQPNC

DAT6580

DO 522 I=1, DIML

IF (IVAR.EQ.1) $\operatorname{READ}(\mathrm{R}, 821)(\mathrm{QBND}(\mathrm{I}, \mathrm{J}), \mathrm{J}=1, \mathrm{DIMW})$

DO $522 \mathrm{~J}=1$, DIMW

IF (IVAR.NE. 1) GO TO 521

DAT6590

$\mathrm{QBND}(\mathrm{I}, \mathrm{J})=\mathrm{QBND}(\mathrm{I}, \mathrm{J}) * \mathrm{FACT}$

DAT6600

DAT6610

DAT6620

GO TO 522

DAT6630

DAT 6640

$521 \mathrm{QBND}(\mathrm{I}, \mathrm{J})=\mathrm{FACT}$

DAT6650

522 CONTINUE

DAT6660

CALL TCOF

DAT6670

DO $523 \mathrm{I}=2$, INO 1

DAT 6680

DO $523 \mathrm{~J}=2$, JNO1

DAT 6690

IF (NODEID(I,J).GE.O) GO TO 523

DAT6700

IF (QBND $(\mathrm{I}, \mathrm{J})$.NE.0.0) GO TO 523

DAT6710

$\mathrm{QB} 1=\mathrm{TC}(\mathrm{I}-1, \mathrm{~J}) *(\mathrm{SURI}(\mathrm{I}, \mathrm{J})-\operatorname{SURI}(\mathrm{I}-1, \mathrm{~J})) * \operatorname{DELX}(\mathrm{J})$

DAT6720

$\mathrm{QB} 2=\mathrm{TC}(\mathrm{I}, \mathrm{J}) *(\operatorname{SURI}(\mathrm{I}, \mathrm{J})-\operatorname{SURI}(\mathrm{I}+1, \mathrm{~J})) * \operatorname{DELX}(\mathrm{J})$

DAT6730

$\mathrm{QB} 3=\mathrm{TR}(\mathrm{I}, \mathrm{J}-1) *(\mathrm{SURI}(\mathrm{I}, \mathrm{J})-\mathrm{SURI}(\mathrm{I}, \mathrm{J}-1)) * \operatorname{DELY}(\mathrm{I})$

DAT6740

$\mathrm{QB} 4=\mathrm{TR}(\mathrm{I}, \mathrm{J}) *(\operatorname{SURI}(\mathrm{I}, \mathrm{J})-\operatorname{SURI}(\mathrm{I}, \mathrm{J}+1)) * \operatorname{DELY}(\mathrm{I})$

$\mathrm{QBT}=\mathrm{QB1}+\mathrm{QB} 2+\mathrm{QB} 3+\mathrm{QB} 4$

$\mathrm{QLK}=0.0$

$\mathrm{QRCH}=0.0$

IF (LEAK.NE.CHK(9)) GO TO 526

DAT6750

DAT6760

DAT6770

DAT6780

IF $(M(I, J) . E Q .0 .0)$ GO TO 526

DAT6790

HED $1=\operatorname{STRT}(\mathrm{I}, \mathrm{J})$

IF (CONVRT . EQ . CHK (7)) HED 1=AMAX1 (STRT $(I, J), \operatorname{TOP}(I, J))$

DAT6800

DAT68 10

QLK=RATE $(I, J) *(\operatorname{RIVER}(I, J)-H E D 1) / M(I, J)$

526 IF (RECH.EQ. CHK (10)) QRCH=QRE $(\mathrm{I}, \mathrm{J})$

$\mathrm{QBND}(\mathrm{I}, \mathrm{J})=\mathrm{QBT}-(\mathrm{QLK}+\mathrm{QRCH}) * \mathrm{DELX}(\mathrm{J}) * \mathrm{DELY}(\mathrm{I})$

523 CONTINUE

IF (IPRN.NE.2) GO TO 3230

JCWT $=10$

JEND $=J C W T$

$\mathrm{JB}=1$

3200 JDIFF=DIMW-JEND

IF (JDIFF.LE.0) JEND=DIMW

DAT6820

DAT6830

DAT6840

DAT6850

DAT 6860

DAT6870

DAT6880

DAT6890

DAT6900

DAT6910

DAT6920

$\operatorname{WRITE}(\mathrm{P}, 3240)(\mathrm{J}, \mathrm{J}=\mathrm{JB}, \mathrm{JEND})$

DAT6930

WRITE $(P, 1190)$

DAT6940

DO $3220 \quad I=1$, DIML

DAT6950

DAT6960

DAT6970

DAT6980

DAT6990

JEND $=\mathrm{JEND}+\mathrm{JCWT}$

DAT7000

GO TO 3200

DAT7010

3230 CONTINUE

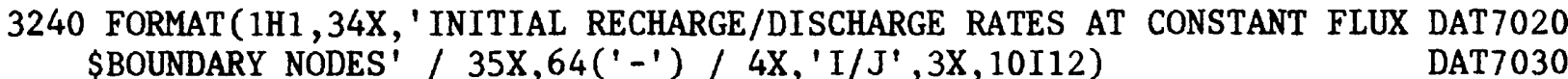

DO $524 \mathrm{I}=1$, DIML

DAT7040

DO $524 \mathrm{~J}=1, \mathrm{DIMW}$

DAT7050

524 IF $(\mathrm{T}(\mathrm{I}, \mathrm{J}) . \mathrm{NE} .0) \quad \mathrm{QBND}(\mathrm{I}, \mathrm{J})=\mathrm{QBND}(\mathrm{I}, \mathrm{J}) /(\mathrm{T}(\mathrm{I}, \mathrm{J}) * \operatorname{DELY}(\mathrm{I}) * \operatorname{DELX}(\mathrm{J}))$

C

525 CONTINUE

DAT7060

DAT 7070

DAT7080

RETURN

DAT7090

C 
Table 1.--Listing of computer program for Laramie County model--Continued

C

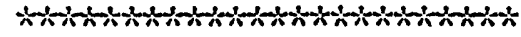

DAT7 130

ENTRY NEWPER

C

DAT7 140

READ (R, 800) KP, KPM1 , NWEL , TMAX, NUMT , CDLT , DELT , ISTOR

DAT7 150

IF (STDY.NE.CHK(15)) GO TO 530

TMAX $=1.0$

NUMT $=1$

$\operatorname{CDLT}=1.0$

DAT7 160

DELT $=24.0$

DAT7 170

$\mathrm{KP}=1$

$\mathrm{KPM} 1=0$

530 CONTINUE

DAT7 180

DAT7 190

DAT7200

DAT7210

DAT7220

DAT7230

$\mathrm{C}$

C ---COMPUTE ACTUAL DELT AND NUMT---

$\mathrm{DT}=\mathrm{DELT} / 24$.

$\mathrm{TM}=0.0$

DO $531 \mathrm{I}=1$, NUMT

DAT7240

$\mathrm{DT}=\mathrm{CDLT} \div \mathrm{DT}$

$\mathrm{TM}=\mathrm{TM}+\mathrm{DT}$

IF (TM.GE.TMAX) GO TO 540

DAT7250

DAT7260

DAT7270

DAT7280

DAT7290

DAT 7300

DAT7310

DAT7320

531 CONTINUE

GO TO 550

540 DELT $=\mathrm{TMAX} / \mathrm{TM} * \mathrm{DELT}$

NUMT $=I$

550 WRITE $(P, 990) \mathrm{KP}$, TMAX, NUMT, DELT, CDLT

DELT $=$ DELT 3600 .

TMAX $=$ TMAX $\div 86400$.

DAT7330

DAT7340

DAT7350

DAT7360

DAT 7370

DAT7380

DAT7390

DAT7400

DAT7410

---READ IN STREAMFLOW ACCOUNTING PARAMETERS---

IF (LEAK. NE . CHK (9) . OR. CONVRT . NE . CHK (7) . OR. NODE. NE . CHK (14))

DAT7420

\$ GO TO 554

IF (ISTOR.LE.0) GO TO 555

NRPR=NRST (ISTOR)

WRITE $(P, 551)$ NRPR

DO $556 \mathrm{I}=1$, NRPR

$\operatorname{NDR}(I)=\operatorname{NDST}($ ISTOR,$I)$

$\operatorname{IRUP}(\mathrm{I})=\operatorname{IRUS}(\mathrm{ISTOR}, \mathrm{I})$

DAT7430

DAT7440

DAT7450

DAT 7460

DAT 7470

DAT7480

$\operatorname{JRUP}(I)=\operatorname{JRUS}(I S T O R, I)$

DAT 7490

$\operatorname{QRIV}(1, I)=Q R S R(I S T O R, I)$

DAT7500

$\operatorname{IRDN}(I)=\operatorname{IRDS}($ ISTOR , I )

DAT7510

$\operatorname{JRDN}(I)=\operatorname{JRDS}($ ISTOR , I)

DAT7520

$\operatorname{QRIV}(2, I)=0.0$

$\operatorname{QRIV}(3, I)=0.0$

$556 \operatorname{WRITE}(\mathrm{P}, 553) \mathrm{I}, \operatorname{NDR}(\mathrm{I}), \operatorname{IRUP}(\mathrm{I}), \operatorname{JRUP}(\mathrm{I}), \operatorname{QRIV}(1, \mathrm{I})$

GO TO 554

$555 \operatorname{READ}(\mathrm{R}, 800)$ NRPR

IF (NRPR.EQ.0) GO TO 554

WRITE $(P, 551)$ NRPR

DAT7530

DAT7540

DAT7550

DAT7560

DAT7570

DAT7580

DAT7590

DAT7600

551 FORMAT ( $4(/), 32 \mathrm{X}$, 'PARAMETERS FOR STREAMFLOW ACCOUNTING PROCEDURE: DAT7610

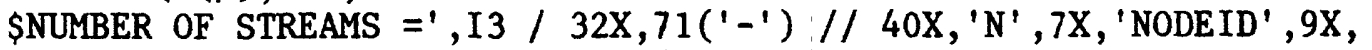
$\$$ ' $\mathrm{I}$ ', $9 \mathrm{X}, \mathrm{I}^{\mathrm{J}} \mathrm{J}, \mathrm{XX}$, 'STREAM INFLOW (CFS)' 1 )

IF (ISTOR.EQ.0) GO TO 557

IS=IABS (ISTOR)

NRST (IS) $=$ NRPR

DAT 7620

DAT7630

DAT7640

DAT7 650

DAT7660

557 DO 552 I=1,NRPR 
Table 1.--Listing of computer program for Laramie County model--Continued

$\operatorname{READ}(R, 800) \operatorname{NDR}(I), \operatorname{IRUP}(I), \operatorname{JRUP}(I), \operatorname{QRIV}(1, I), \operatorname{IRDN}(I), \operatorname{JRDN}(I)$

$\operatorname{QRIV}(2, I)=0.0$

$\operatorname{QRIV}(3, I)=0.0$

IF (ISTOR.EQ.0) GO TO 552

IS $=$ IABS (ISTOR)

$\operatorname{NDST}(I S, I)=\operatorname{NDR}(I)$

$\operatorname{IRUS}(I S, I)=\operatorname{IRUP}(I)$

$\operatorname{JRUS}(I S, I)=\operatorname{JRUP}(I)$

$\operatorname{QRSR}(I S, I)=Q R I V(1, I)$

$\operatorname{IRDS}(I S, I)=\operatorname{IRDN}(I)$

$\operatorname{JRDS}(\mathrm{IS}, \mathrm{I})=\operatorname{JRDN}(\mathrm{I})$

552 WRITE $(P, 553) I, \operatorname{NDR}(I), \operatorname{IRUP}(I), \operatorname{JRUP}(I), Q R I V(1, I)$

553 FORMAT ( $36 \mathrm{X}, \mathrm{I} 5,5 \mathrm{X}, \mathrm{I} 8,2(5 \mathrm{X}, \mathrm{I} 5)$, 1PE 22.3)

554 CONTINUE

$\mathrm{C}$

C ---INITIALIZE SUMP, STRT, SL, WELL AND WR---

WRITE $(P, 1000)$ NWEL

IF (KP.GT.KPM1) SUMP=0.

DO 571 I=1, DIML

DO $571 \mathrm{~J}=1$, DIMW

IF (KP.EQ.KPM1) GO TO 560

$\operatorname{STRT}(I, J)=\operatorname{PHI}(I, J)$

560 IF (LEAK.NE.CHK(9)) GO TO 570

IF $(M(I, J) . E Q .0$.$) GO TO 570$

$\operatorname{SL}(I, J)=\operatorname{RATE}(I, J) / M(I, J) *(\operatorname{RIVER}(I, J)-\operatorname{STRT}(I, J))$

IF (NODE.NE.CHK(14)) GO TO 570

IF (CONVRT.NE.CHK(7)) GO TO 570

$\mathrm{ND}=\operatorname{IABS}(\operatorname{NODE} I D(I, J))$

IF (ND.EQ.0) GO TO 570

$\operatorname{TL}(I, J)=\operatorname{RATE}(I, J) / M(I, J)$

IF (NRPR.NE.0) GO TO 562

DO $561 \mathrm{NR}=1$, NRIV

IF (ND.NE.NODRV(NR)) GO TO 561

$\operatorname{TL}(I, J)=0.0$

$\operatorname{SL}(I, J)=0.0$

561 CONTINUE

GO TO 570

562 DO 563 NR=1, NRPR

IF (ND.EQ.NDR(NR)) GO TO 570

563 CONTINUE

DO 564 NR=1, NRIV

IF (ND.NE.NODRV(NR)) GO TO 564

$\operatorname{TL}(I, J)=0.0$

$\operatorname{SL}(I, J)=0.0$

564 CONTINUE

570 IF (NODE.EQ.CHK(14).AND.NODEID $(I, J)$. GE. 0) WELL $(I, J)=0.0$

571 CONTINUE

IF (NW.EQ.0) GO TO 590

DO $580 \quad I=1, N W$

580 WR(I) $=0$.

C

590 IF (NWEL.EQ.0) GO TO 630

C ---READ AND WRITE WELL PUMPING RATES AND WELL RADII---

$\mathrm{KW}=0$

DO 620 II $=1$, NWEL
DAT7680

DAT7690

DAT7700

DAT 7710

DAT 7720

DAT 7730

DAT7740

DAT 7750

DAT7760

DAT 7770

DAT 7780

DAT 7790

DAT 7800

DAT 7810

DAT7820

DAT7830

DAT7840

DAT7850

DAT 7860

DAT7870

DAT 7880

DAT 7890

DAT 7900

DAT79 10

DAT 7920

DAT7930

DAT 7940

DAT7950

DAT7960

DAT7970

DAT7980

DAT7990

DAT8000

DAT8010

DAT8020

DAT8030

DAT 8040

DAT 8050

DAT 8060

DAT 8070

DAT 8080

DAT 8090

DAT 8100

DAT 8110

DAT8120

DAT 8130

DAT 8140

DAT 8150

DAT 8160

DAT 8170

DAT 8180

DAT 8190

DAT 8200

DAT8210

DAT8220 
Table 1.--Listing of computer program for Laramie County model--Continued

IF (ISTOR.GT.0) GO TO 621

READ $(R, 800) I, J$, WELL $(I, J)$, RADIUS

IF (ISTOR.EQ.0) GO TO 622

IW=IABS (ISTOR)

IWST $(I W, I I)=I$

JWST $(I W, I I)=\mathrm{J}$

WLST $(I W, I I)=W E L L(I, J)$

RADST $(I W, I I)=$ RADIUS

GO TO 622

621 I=IWST (ISTOR, II)

$\mathrm{J}=\mathrm{JWST}$ (ISTOR, II)

WELL $(I, J)=W L S T$ (ISTOR, II )

RADIUS=RADST $($ ISTOR , II $)$

DAT 8230

DAT 8240

DAT 8250

DAT8260

DAT 8270

DAT8280

DAT 8290

DAT 8300

DAT 8310

DAT8320

DAT 8330

DAT 8340

DAT 8350

622 CONTINUE

DAT 8360

IF (RADIUS.EQ.0.) GO TO 600

DAT8370

$\mathrm{KW}=\mathrm{KW}+1$

DAT 8380

IF (KW.GT.NW) GO TO 600

DAT8390

$\operatorname{NWR}(K W, 1)=I$

DAT8400

$\operatorname{NWR}(K W, 2)=J$

DAT 8410

$W R(K W)=R A D I U S$

DAT 8420

WRITE $(P, 1010)$ I, J, WELL(I, J), WR(KW)

DAT 8430

GO TO 610

DAT 8440

600 WRITE $(P, 1010) I, J, \operatorname{WELL}(I, J)$

DAT 8450

$610 \operatorname{WELL}(\mathrm{I}, \mathrm{J})=\operatorname{WELL}(\mathrm{I}, \mathrm{J}) /(\operatorname{DELX}(\mathrm{J}) * \operatorname{DELY}(\mathrm{I}))$

DAT 8460

C

620 CONTINUE

DAT 8470

DAT 8480

C

630 IF (RECH.NE.CHK(10)) GO TO 634

QRE (RECHARGE RATE)

DAT 8490

DAT8500

READ $(R, 800)$ FACT, IVAR, IPRN

DAT 8510

IF (FACT.EQ.0.0) GO TO 634

DAT8520

IF (IVAR.EQ.1.AND.IPRN.EQ.0) WRITE (P,950)

DAT 8530

DO 633 I=1, DIML

IF (IVAR.EQ.1) READ (R,820) (QRE (I, J), J=1,DIMW)

DAT 8540

DO $632 \mathrm{~J}=1$, DIMW

IF (IVAR.NE.1) GO TO 631

DAT 8550

DAT8560

$\mathrm{QRE}(\mathrm{I}, \mathrm{J})=\mathrm{QRE}(\mathrm{I}, \mathrm{J}) * \mathrm{FACT}$

DAT8570

GO TO 632

DAT 8580

DAT 8590

$631 \mathrm{QRE}(\mathrm{I}, \mathrm{J})=\mathrm{FACT}$

DAT 8600

DAT 8610

633 IF (IVAR.EQ.1.AND.IPRN.EQ.0) WRITE (P,860) I, (QRE (I,J), J=1,DIMW)

IF (IVAR.NE. 1) WRITE (P,750) FACT

IF (IVAR.EQ.0.OR.IPRN.NE.2) GO TO 3330

DAT 8620

DAT 8630

DAT 8640

$\mathrm{JCWT}=10$

DAT 8650

JEND $=\mathrm{JCWT}$

DAT 8660

$\mathrm{JB}=1$

3300 JDIFF=DIMW - JEND

IF (JDIFF.LE. 0) JEND=DIMW

DAT 8670

DAT 8680

DAT 8690

WRITE $(P, 3340) \quad(J, J=J B, J E N D)$

DAT 8700

WRITE $(P, 1190)$

DAT 8710

DO $3320 \quad I=1$, DIML

DAT 8720

3320 WRITE $(P, 2250) \quad I,(Q R E(I, J), J=J B, J E N D)$

DAT8730

IF (JEND.EQ.DIMW) GO TO 3330

DAT 8740

$\mathrm{JB}=\mathrm{JEND}+1$

DAT 8750

JEND $=\mathrm{JEND}+\mathrm{JCWT}$

DAT 8760

GO TO 3300

DAT 8770 
Table 1.--Listing of computer program for Laramie County model--Continued

3330 CONTINUE

3340 FORMAT (1H1,60X, 'RECHARGE RATE' / 61X,13('-') / 4X, 'I/J', 3X,

\$ 10I12)

634 RETURN

DAT 8780

DAT 8790

DAT 8800

DAT 8810

C

C

FORMATS :

DAT 8830

FORMATS:

DAT 8840

DAT 8850

-DAT8860

DAT 8870

640 FORMAT (' 0 ', 63X, 'STARTING HEAD $='$, G15.7)

DAT 8880

650 FORMAT (' 0 ', $57 \mathrm{X}$, 'STORAGE COEFFICIENT $=', \mathrm{G} 15.7$ )

DAT 8890

660 FORMAT (' 0 ', $62 \mathrm{X}$, , SPECIFIC YIELD $=$ ', G15.7)

DAT8900

670 FORMAT (' 0 ', 37X, 'HYDRAULIC CONDUCTIVITY OF CONFINING BED $=$ ', G15.7)DAT8910

680 FORMAT (' 0 ', $66 \mathrm{X}$, 'RIVER HEAD $=$ ', G15.7)

690 FORMAT (' 0 ' , 53X,' CONFINING BED THICKNESS $=$ ' , G15.7)

700 FORMAT (' 0 ' , 62X,' TRANSMISSIVITY $=$ ', G15.7)

710 FORMAT (' 0 ', 46X, 'AQUIFER HYDRAULIC CONDUCTIVITY =', G15.7)

720 FORMAT (' 0 ', 60X, 'BOTTOM ELEVATION $=$ ' , G15.7)

730 FORMAT (' 0 ', $63 \mathrm{X}$, ' TOP ELEVATION $='$, G15.7)

740 FORMAT (' 0 ' , 62X, 'LAND ELEVATION $=$ ', G15.7)

750 FORMAT (' 0 ', $63 \mathrm{X}$, ' RECHARGE RATE $=$ ', G15.7)

760 FORMAT ( ' 0 ' , 72X, 'DELX $=$ ', G15.7)

770 FORMAT (' 0 ', $72 \mathrm{X}$, ' DELY $=$ ', G15.7)

780 FORMAT (' 1 ',60X,'STARTING HEAD MATRIX'/61X,20(' $-'$ '))

DAT 8920

DAT 8930

DAT 8940

DAT 8950

DAT 8960

DAT 8970

DAT 8980

DAT 8990

DAT9000

790 FORMAT (' 1 ',40X,' CONTINUATION - HEAD AFTER ',G20.7,' SEC PUMPING

$\left.1^{\prime} / 42 \mathrm{X}, 58\left('-{ }^{\prime}\right)\right)$

800 FORMAT $(8 \mathrm{G} 10.0)$

810 FORMAT $(A 4,6 \mathrm{X}, 5 \mathrm{G} 10.0, \mathrm{~A} 8)$

820 FORMAT (20F4.0)

821 FORMAT (1P8E10.3)

822 FORMAT (16F5.0)

830 FORMAT ( $1 \mathrm{H} 1,61 \mathrm{X}, 11 \mathrm{HRATE}$ MATRIX/62X,11('-'))

DAT9010

DAT 9020

840 FORMAT (' 0 ' ,51X, 'NUMBER OF PUMPING PERIODS $=$ ', I5/49X, 'TIME STEPS BDAT9110

1ETWEEN PRINTOUTS $=$ ' , I5//51X, 'ERROR CRITERIA FOR CLOSURE =' , G15.7/3DAT9120

29X, 'MAXIMUM PERMITTED NUMBER OF ITERATIONS =' , I5/41X,'

STEDAT 9130

3ADY STATE ERROR CRITERIA $='$, G15.7//44X,' SPECIFIC STORAGE OF CONFINDAT9140

4ING BED $=$ ', G15.7/54X, 'EVAPOTRANSPIRATION RATE =', G15.7/56X, 'EFFECTDAT9150

5IVE DEPTH OF ET $='$, G15.7//22X, 'MULTIPLICATION FACTOR FOR TRANSMISSDAT9160

6IVITY IN X DIRECTION $=$ ', G15.7/63X, 'IN Y DIRECTION =' ,G15.7)

DAT 9170

850 FORMAT ( $\left.1 \mathrm{H} 1,64 \mathrm{X}, 23 \mathrm{HTRANSMISSIVITY} \mathrm{MATRIX} / 65 \mathrm{X}, 21\left(^{\prime}-^{\prime}\right)\right)$

860 FORMAT (1H0, I5 , 1P10E 12.3/(1H , 5X, 1P10E 12.3))

870 FORMAT (' 0 ' , I2 , 2X, 16F8.1/(5X,16F8.1))

880 FORMAT (1HO, I5, 14F9.5/(1H ,5X,14F9.5))

890 FORMAT (1H1,54X,26HSTORAGE COEFFICIENT MATRIX/55X,26(' $\left.{ }^{\prime}{ }^{\prime}\right)$ )

900 FORMAT (8F10.4)

910 FORMAT ( $1 \mathrm{H} 1,52 \mathrm{X}, 29 \mathrm{HHYDRAULIC}$ CONDUCTIVITY MATRIX/53X,29( $\left.{ }^{\prime}-^{+}\right)$)

DAT 9180

DAT9 190

DAT9200

DAT9210

DAT9220

DAT9230

$\left.1\left({ }^{\prime}-1\right)\right)$

930 FORMAT (' 1 ',53X,' ELEVATION OF TOP OF AQUIFER' $/ 55 \mathrm{X}, 27\left({ }^{\prime}-1\right)$ )

940 FORMAT (' 1 ',54X, 'ELEVATION OF LAND SURFACE' $\left./ 55 \mathrm{X}, 25\left('-{ }^{\prime}\right)\right)$

950 FORMAT (' 1 ', $57 \mathrm{X}$, 'AREAL RECHARGE RATE'/58X,19('-'))

ODAT9250

DAT9260

DAT9270

DAT9280

960 FORMAT ( $1 \mathrm{H} 1,46 \mathrm{X}, 40 \mathrm{HGRID}$ SPACING IN PROTOTYPE IN X DIRECTION/47X,40DAT9300 $1('-') / /(' 0 ', 12 \mathrm{~F} 10.0))$

DAT9310

970 FORMAT (1H-,46X,40HGRID SPACING IN PROTOTYPE IN Y DIRECTION/47X,40DAT9320 
Table 1.-- Listing of computer program for Laramie County model--Continued

$\left.1\left({ }^{\prime}-{ }^{\prime}\right) / /\left('{ }^{\prime}, 12 \mathrm{~F} 10.0\right)\right)$

DAT9330

980 FORMAT ( $1 \mathrm{H} 1,60 \mathrm{X}, 17 \mathrm{HRIVER}$ HEAD MATRIX/61X, $17\left({ }^{\prime}-{ }^{\prime}\right)$ )

DAT9340

990 FORMAT (' 1 ',50X,' PUMPING PERIOD NO.' , I4, ': ',F10.2,' DAYS'/51X, 38('DAT9350

$\left.1-^{\prime}\right) / / 53 \mathrm{X},{ }^{\prime}$ NUMBER OF TIME STEPS $=1$, I6//59X, 'DELT IN HOURS $={ }^{\prime}, \mathrm{F} 10.3 / /$ DAT9360

253X,'MULTIPLIER FOR DELT $=', F 10.3$ )

DAT 9370

1000 FORMAT $\left('-', 63 \mathrm{X}, \mathrm{I} 4\right.$, ' WELLS' $/ 65 \mathrm{X}, 9('-') / / 50 \mathrm{X},{ }^{\prime} \mathrm{I}$ ', $9 \mathrm{X}$, ' $\mathrm{J}$ PUMPING RDAT9380

IATE WELL RADIUS'/)

DAT9390

1010 FORMAT $(41 \mathrm{X}, 2 \mathrm{I} 10,2 \mathrm{~F} 13.4)$

1020 FORMAT $(' 1$ ',55X, 'CONF INING BED THICKNESS'/56X,23('-'))

DAT 9400

1030 FORMAT (4G20.10)

DAT 9410

1040 FORMAT (' 0 ' 30X''ON ALPHAMERTC MAP.' / $40 \mathrm{X}$ 'MUITIPIICATION FACTOR FODAT9430

$1 R$ X DIMENSION $=\prime$, G15.7/40X, 'MULTIPLICATION FACTOR FOR Y DIMENSION DAT9440

$2=', \mathrm{G} 15.7 / 55 \mathrm{X}$, 'MAP SCALE IN UNITS OF ',A11/50X,' NUMBER OF ',A8,' PDAT9450

3ER INCH $='$, G15.7/43X, 'MULTIPLICATION FACTOR FOR DRAWDOWN =' , G15.7/DAT9460

447X, 'MULTIPLICATION FACTOR FOR HEAD =', G15.7)

1050 FORMAT $\left.\left(1 \mathrm{H} 1,56 \mathrm{X}, 21 \mathrm{HSPECIFIC} \mathrm{YIELD} \mathrm{MATRIX/57X,21('}{ }^{\prime}{ }^{\prime}\right)\right)$

DAT9470

1060 FORMAT (20I4)

1065 FORMAT (' 1 ' ,52X, 'HYDRAULIC CONDUCTIVITY MATRIX'///8X,55I2/)

DAT 9480

1070 FORMAT (' ' , 2X, I2 , 3X, 55 I2, I4)

1080 FORMAT ('0', $7 \mathrm{X}, 55 \mathrm{I} 2$ )

DAT 9490

DAT9500

DAT 9510

1190 FORMAT ( $1 \mathrm{HO}$ )

DAT9520

END

DAT 9530

SUBROUTINE STEP(PHI , KEEP , STRT , SURI , T , WELL , PERM , BOTTOM, TOP , DELX, DDNSTP 10

1 , DELY , WR , NWR , NODE ID , QBND)

STP 20

C

C

C

C

C

C

INITIALIZE DATA FOR TIME STEP, CHECK FOR STEADY STATE,

STP 30

PRINT AND PUNCH RESULTS

STP 40

STP 50

SPECIFICATIONS :

STP 70

COMMON /SARRAY/ TEST3(102),VF4(11), CHK(15), ITST(102),JTST(102) STP 90

COMMON /SPARAM/ WATER, CONVRT, EVAP, CHCK, PNCH, NUM, HEAD, CONTR, EROR, LESTP 100

$1 \mathrm{AK}, \mathrm{RECH}, \mathrm{SIP}, \mathrm{U}, \mathrm{SS}$, TT , TMIN , ETDIST , QET , ERR , TMAX , CDLT , HMAX, YDIM, WIDTH , STP 110

2NUMS , LSOR , ADI , DELT , SUM , SUMP , SUBS , STORE , TEST , ETQB , ETQD , FACTX, FACTY , STP 120

3IERR , KOUNT , IF INAL , NUMT , KT , KP , NPER , KTH , ITMAX, LENGTH , NWEL , NW , DIML , DISTP 130

4MW, JNO1, INO1 , R , P , PU, I , J , NODE , STDY , KPH, IQPNC

STP 140

COMMON /CK/ ETFLXT, STORT, QRET, CHST, CHDT, FLUXT, PUMPT, CFLUXT, FLXNT STP 150

COMMON /ARSIZE/ IZ, JZ, IP , JP, IR, JR, IC , JC, IL , JL, IS , JS, IH, IMAX $\quad$ STP 160

$\$, I U, J U$

STP 170

COMMON /PR/ XLABEL (3), YLABEL (6), TITLE (5), XN1, MESUR, PRNT (122) , BLANKSTP 180 $1(60), \operatorname{DIGIT}(122), \mathrm{VF} 1(6), \mathrm{VF} 2(6), \mathrm{VF} 3(7), \mathrm{XSCALE}, \mathrm{DINCH}, \operatorname{SYM}(17), \mathrm{XN}(100), \mathrm{STP} 190$ $2 \mathrm{YN}(13), \mathrm{NA}(4), \mathrm{N} 1, \mathrm{~N} 2, \mathrm{~N} 3$, YSCALE , FACT1 , FACT2

STP 200

COMMON /STREAM/ NRIV,NRPR, NODRV (20), NDR(20), NMRV (20), QRIV (3, 20),

C

$\$ \operatorname{IRUP}(20), \operatorname{JRUP}(20), \operatorname{IRDN}(20), \operatorname{JRDN}(20)$

STP 210

STP 220

STP 230

DIMENSION PHI $(I Z, J Z), \operatorname{KEEP}(I Z, J Z), \operatorname{STRT}(I Z, J Z), \operatorname{SURI}(I Z, J Z), T(I Z, S T P 240$ $1 \mathrm{JZ}), \operatorname{BOTTOM}(I P, J P), \operatorname{WELL}(I Z, J Z), \operatorname{PERM}(I P, J P)$, TOP (IC,JC), DELX(JZ)STP 250 2, DDN(JZ), DELY (IZ), WR(IH), NWR(IH, 2), NODEID (IU, JU),

C $\$$ QBND (IU, JU)

REAL *8PHI, DBLE, DABS , TEST2, DMAX 1 , XLABEL, YLABEL, XN1, MESUR, TITLE STP 260 STP 270 STP 280 REAL *4MINS, M, KEEP STP 290 INTEGER R, P, PU, DIML , DIMW, CHK, WATER, CONVRT , EVAP , CHCK, PNCH, NUM, HEAD , STP 310 1CONTR , LEAK, RECH , SIP , ADI , STDY

STP 320

DATA PIE/3.141593/

STP 330 
Table 1.--Listing of computer program for Laramie County model--Continued

RETURN

STP 340

$$
\begin{aligned}
& c \\
& c \\
& c \\
& c
\end{aligned}
$$

C

C

C

C

C

\section{---START A NEW TIME STEP---}

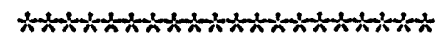

ENTRY NEWSTP

B

$\mathrm{KT}=\mathrm{KT}+1$

KOUNT $=0$

DO $10 \quad I=1$, DIML

DO $10 \mathrm{~J}=1$, DIMW

IF (NODE. NE.CHK (14)) GO TO 10

IF (NODEID $(\mathrm{I}, \mathrm{J})$.LT.0) $\operatorname{WELL}(\mathrm{I}, \mathrm{J})=\mathrm{T}(\mathrm{I}, \mathrm{J}) \div \mathrm{QBND}(\mathrm{I}, \mathrm{J})$

$10 \operatorname{KEEP}(\mathrm{I}, \mathrm{J})=\operatorname{PHI}(\mathrm{I}, \mathrm{J})$

DELT $=$ CDLT $* D E L T$

SUM $=$ SUM+DELT

SUMP $=$ SUMP+DELT

DAYSP $=S U M P / 86400$.

YRSP $=$ DAYSP $/ 365$.

HRS $=S U M / 3600$.

MINS $=H R S * 60$.

DAYS $=$ HRS $/ 24$.

YRS $=$ DAYS $/ 365$.

RETURN

STP 350

STP 360

STP 370

STP 380

STP 390

STP 400

STP 410

STP 420

STP 430

STP 440

STP 450

STP 460

STP 470

STP 480

STP 490

STP 500

STP 510

STP 520

STP 530

STP 540

STP 550

STP 560

STP 570

C

C

C

C

C

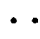

\section{---CHECK FOR STEADY STATE---}

*

ENTRY STEADY

C

TEST2 $=0$.

DO $20 \mathrm{I}=2$, INO 1

DO $20 \mathrm{~J}=2$, JNO 1

$20 \operatorname{TEST} 2=\operatorname{DMAX} 1(\operatorname{TEST} 2, \operatorname{DABS}(\operatorname{DBLE}(\operatorname{KEEP}(I, J))-\operatorname{PHI}(I, J)))$

IF (TEST2.GE.EROR) GO TO 30

WRITE $(P, 280) \mathrm{KT}$

IFINAL $=1$

GO TO 40

C

30 IF (KT.EQ.NUMT) IFINAL=1

STP 580

STP 590

STP 600

STP 610

STP 620

STP 630

STP 640

STP 650

STP 660

STP 670

STP 680

STP 690

STP 700

STP 710

STP 720

STP 730

STP 740

STP 750

STP 760

STP 770

STP 780

STP 790

STP 800

STP 810

STP 820

KOUNT $=$ KOUNT -1

STP 830

C

GO TO 60

STP 840

STP 850

STP 860

50 IF (MOD (KT, KTH) .NE . 0.AND. IF INAL. NE. 1) RETURN

60 WRITE (P , 290) KT, DELT , SUM, MINS, HRS , DAYS, YRS, DAYSP, YRSP , KOUNT

IF (CHCK.EQ.CHK(5)) CALL CWRITE

STP 880 
Table 1.--Listing of computer program for Laramie County model--Continued

IF (TT.NE.0.) WRITE $(P, 270)$ TMIN,TT

KOUNT $=$ KOUNT +1

WRITE $(P, 250)$

DO $65 \mathrm{~J}=1$, KOUNT

$65 \operatorname{WRITE}(\mathrm{P}, 255) \operatorname{ITST}(\mathrm{J}), \operatorname{JTST}(\mathrm{J}), \operatorname{TEST} 3(\mathrm{~J})$

WRITE $(\mathrm{P}, 240)$ TEST2

IF (CONTR.NE.CHK(3)) GO TO 70

C IF (FACT1.NE.0.) CALL PRNTA(1)

C IF (FACT2.NE.0.) CALL PRNTA(2)

70 IF (KPH.EQ.0) GO TO 71

IF (MOD (KP, KPH).NE. 0) GO TO 95

STP 890

STP 900

STP 910

STP 920

STP 930

STP 940

STP 950

STP 960

STP 970

STP 980

STP 990

71 IF (HEAD.NE.CHK(8)) GO TO 90

STP 1000

C

---PRINT HEAD MATRIX---

STP 1010

$\mathrm{JCWT}=15$

STP 1020

JEND $=$ JCWT

STP 1030

$\mathrm{JB}=1$

2100 JDIFF=DIMW-JEND

IF (JDIFF.LE.0) JEND=DIMW

WRITE $(P, 2140) \quad(J, J=J B, J E N D)$

STP 1040

STP 1050

STP 1060

STP 1070

WRITE $(P, 1190)$

DO $2120 \quad I=1$,DIML

2120 WRITE $(P, 2150) I,(P H I(I, J), J=J B, J E N D)$

IF (JEND.EQ.DIMW) GO TO 2130

$\mathrm{JB}=\mathrm{JEND}+1$

JEND $=\mathrm{JEND}+\mathrm{JCWT}$

GO TO 2100

2130 CONTINUE

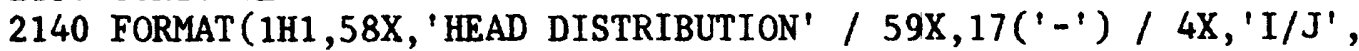
$\$ 3 X, 15 I 8)$

2150 FORMAT $(I 5,5 X, 15 F 8.1)$

90 IF (NUM.NE.CHK(4)) GO TO 120

C ---PRINT FINAL SATURATED THICKNESS---

IF (WATER.NE.CHK(2)) GO TO 440

JCWT $=15$

JEND $=J C W T$

$\mathrm{JB}=1$

410 JDIFF=DIMW-JEND

IF (JDIFF.IE.0) JEND=DIMW

$\operatorname{WRITE}(\mathrm{P}, 430) \quad(\mathrm{J}, \mathrm{J}=\mathrm{JB}, \mathrm{JEND})$

WRITE $(P, 1190)$

DO $420 \quad I=1$, DIML

STP 1080

STP 1090

STP 1100

STP 1110

STP 1120

STP 1130

STP 1140

STP 1150

STP 1160

STP 1170

STP 1180

STP 1190

STP1200

STP 1210

STP 1220

STP 1230

STP 1240

STP 1250

STP 1260

STP 1270

STP 1280

STP 1290

STP 1300

DO $421 \mathrm{~J}=\mathrm{JB}$, JEND

STP1310

STP1320

STP1330

STP 1340

420 WRITE $(P, 2350)$ I, (DDN $(J), J=J B, J E N D)$

STP1350

$\mathrm{JB}=\mathrm{JEND}+1$

STP1360

JEND $=$ JEND + JCWT

STP 1370

GO TO 410

440 CONTINUE

430 FORMAT $(1 \mathrm{H} 1,54 \mathrm{X}$, 'FINAL SATURATED THICKNESS' / 55X,25('-') /

STP 1380

$\left.\$ 4 X, ' I / J^{\prime}, 3 X, 15 I 8\right)$

C

$\begin{array}{lll}\text { C } & & \text { STP1410 } \\ \text { C --PRINT BOUNDARY FLUX AND STREAMFLOW ARRAY, QBND(I,J)--- } & \text { STP1420 }\end{array}$

STP 1390

STP 1400

STP 1410

IF (NRPR.EQ.0) GO TO 95 
Table 1.--Listing of computer program for Laramie County model--Continued

JCWT $=10$

$\operatorname{STP} 1440$

$\mathrm{JEND}=\mathrm{JCWT}$

STP 1450

$\mathrm{JB}=1$

3200 JDIFF=DIMW-JEND

IF (JDIFF.LE .0) JEND=DIMW

STP 1460

WRITE $(P, 3240)(J, J=J B, J E N D)$

STP 1470

WRITE $(P, 1190)$

DO $3220 \quad \mathrm{I}=1$, DIML

DO $3219 \mathrm{~J}=\mathrm{JB}, \mathrm{JEND}$

$\operatorname{DDN}(\mathrm{J})=\mathrm{QBND}(\mathrm{I}, \mathrm{J})$

$\operatorname{IF}(\operatorname{NODEID}(\mathrm{I}, \mathrm{J}) . \mathrm{LT} .0) \operatorname{DDN}(\mathrm{J})=0.0$

STP 1480

STP 1490

STP 1500

3219 CONTINUE

$3220 \operatorname{WRITE}(\mathrm{P}, 3250)$ I , (DDN $(\mathrm{J}), \mathrm{J}=\mathrm{JB}, \mathrm{JEND})$

STP 1510

STP 1520

STP 1530

STP 1540

IF (JEND.EQ.DIMW) GO TO 3230

STP 1550

$\mathrm{JB}=\mathrm{JEND}+1$

STP 1560

JEND $=\mathrm{JEND}+\mathrm{JCWT}$

STP 1570

GO TO 3200

STP 1580

STP 1590

STP 1600

3230 CONTINUE

STP 1610

3240 FORMAT (1H1 , 49X, 'BOUNDARY FLUX AND STREAMFLOW ARRAY' / 50X,34(' -')

$\$ / 4 X, ' I / J ', 3 X, 10 I 12)$

STP 1620

STP 1630

STP 1640

STP 1650

WRITE $(P, 695)$

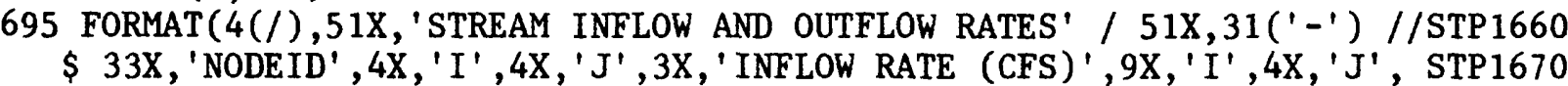

$\$ 2 X, '$ OUTFLOW RATE (CFS)' //)

STP 1680

DO $96 \mathrm{NR}=1$, NRPR

$96 \operatorname{WRITE}(\mathrm{P}, 696) \mathrm{NDR}(\mathrm{NR}), \operatorname{IRUP}(\mathrm{NR}), \operatorname{JRUP}(\mathrm{NR}), \mathrm{QRIV}(3, \mathrm{NR}), \operatorname{IRDN}(\mathrm{NR})$,

$\$ \operatorname{JRDN}(\mathrm{NR}), \mathrm{QRIV}(2, \mathrm{NR})$

696 FORMAT (29X, I10,2I5, 1PE20.3,5X,2I5, 1PE20.3)

C

95 IF (NUM.NE.CHK(4)) GO TO 120

STP 1690

STP1700

STP1710

STP1720

STP 1730

STP 1740

---PRINT DRAWDOWN---

STP1750

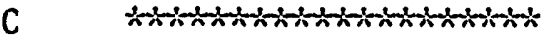

STP 1760

ENTRY DRDN

STP1770

C

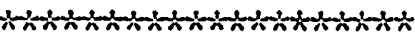

STP 1780

JCWT $=15$

STP 1790

JEND $=J C W T$

STP 1800

$\mathrm{JB}=1$

2300 JDIFF $=$ DIMW-JEND

IF (JDIFF.LE.0) JEND=DIMW

WRITE $(P, 2340) \quad(J, J=J B, J E N D)$

STP 1810

STP 1820

WRITE $(P, 1190)$

DO 2320 I=1, DIML

DO $100 \mathrm{~J}=\mathrm{JB}, \mathrm{JEND}$

$\operatorname{DDN}(J)=0.0$

IF $(\operatorname{PHI}(I, J) . N E .0 .0) \operatorname{DDN}(J)=\operatorname{SURI}(I, J)-\operatorname{PHI}(I, J)$

STP 1830

STP 1840

STP 1850

STP 1860

STP 1870

STP1 1880

100 CONTINUE

2320 WRITE $(P, 2350)$ I, (DDN (J), J=JB, JEND)

STP1890

STP1900

STP 1910

IF (JEND.EQ.DIMW) GO TO 2330

STP 1920

$\mathrm{JB}=\mathrm{JEND}+1$

JEND $=$ JEND $+J C W T$

STP1930

GO TO 2300

STP 1940

STP1950

2330 CONTINUE

STP 1960

2340 FORMAT $(1 \mathrm{H1}, 62 \mathrm{X}$, ' DRAWDOWN' / 63X,8('-') / 4X, ' I/J', 3X, 15I8)

2350 FORMAT (I5, 5X,15F8.2)

STP 1970

STP 1980 
Table 1.--Listing of computer program for Laramie County model--Continued

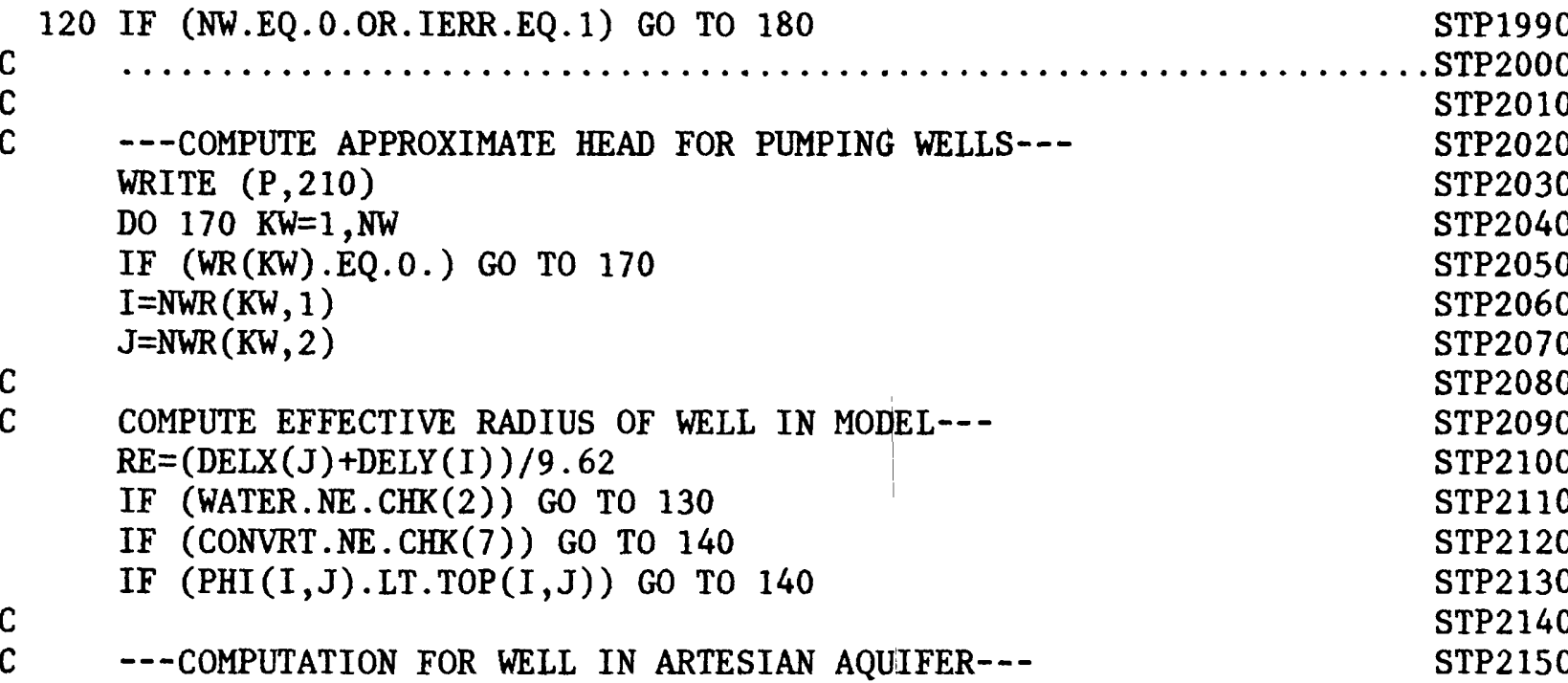

$130 \mathrm{HW}=\operatorname{PHI}(\mathrm{I}, \mathrm{J})+\mathrm{WELL}(\mathrm{I}, \mathrm{J}) * \operatorname{ALOG}(\mathrm{RE} / \mathrm{WR}(\mathrm{KW})) /(2 . * \mathrm{PIE} * \mathrm{~T}(\mathrm{I}, \mathrm{J})) * \mathrm{DELX}(\mathrm{J}) * \mathrm{DELYSTP} 2160$

$1(\mathrm{I})$

GO TO 160

STP2 170

STP2 180

C

C ---COMPUTATION FOR WELL IN WATER TABLE AQUIFER

STP2190

STP 2200

$140 \operatorname{HED}=\mathrm{PHI}(\mathrm{I}, \mathrm{J})-\mathrm{BOTTOM}(\mathrm{I}, \mathrm{J})$

STP2210

$A R G=H E D * H E D+W E L L(I, J) * A L O G(R E / W R(K W)) /(P I E * P E R M(I, J)) * D E L X(J) * D E L Y S T P 2220$

$1(\mathrm{I})$

IF (ARG.GT.0.) GO TO 150

STP2230

WRITE $(P, 220)$ I,$J$

STP2240

GO TO 170

STP2250

STP2260

$150 \mathrm{HW}=\mathrm{SQRT}(\mathrm{ARG})+\mathrm{BOTTOM}(\mathrm{I}, \mathrm{J})$

STP2270

C

C ---COMPUTE DRAWDOWN AT THE WELL AND PRINT RESULTS---

STP2280

160 DRAW=SURI $(I, J)-H W$

STP2290

WRITE $(P, 200)$ I , J, WR(KW), HW, DRAW

STP2300

170 CONTINUE

STP2310

180 IF (IERR.NE.2) RETURN

STP2320

IF (PNCH.NE.CHK (1)) STOP

STP2330

STP2340

C

STP2350

C

C

---PUNCHED OUTPUT---

STP2360

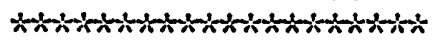

STP 2370

ENTRY PUNCH

STP 2380

A

STP 2390

WRITE (PU 310) SUM SUMP ,PUMPT, CFLUXT, QRET, CHST, CHDT , FIUXT STORT , ETSTP2410

IFLXT, FLXNT

DO $190 \quad \mathrm{I}=1$, DIML

190 WRITE (PU, 300) (PHI ( I, J), J=1, DIMW)

IF (NODE. NE.CHK (14)) GO TO 194

IF (IQPNC.EQ.0) GO TO 194

DO 191 I=1,DIML

DO $191 \mathrm{~J}=1$, DIMW

IF (QBND(I,J).NE.0.0) GO TO 192

STP2 400

191 CONTINUE

GO TO 194

STP2420

STP2430

STP2440

STP 2450

STP2460

STP 2470

STP 2480

STP 2490

STP 2500

STP25 10

192 DO 193 I=1, DIML

STP2520

DO $196 \mathrm{~J}=1$, DIMW

STP2530 
Table 1.--Listing of computer program for Laramie County model--Continued

$\operatorname{DDN}(\mathrm{J})=0.0$

IF (NODEID $(I, J) . G E .0)$ GO TO 196

STP 2540

$\operatorname{DDN}(\mathrm{J})=\mathrm{QBND}(\mathrm{I}, \mathrm{J}) * \mathrm{~T}(\mathrm{I}, \mathrm{J}) * \operatorname{DELX}(\mathrm{J}) * \mathrm{DELY}(\mathrm{I})$

STP 2550

196 CONTINUE

193 WRITE(PU, 195) (DDN(J), J=1,DIMW)

195 FORMAT (1P8E 10.3)

194 CONTINUE

IF (KOUNT.GT. ITMAX) STOP

RETURN

STP 2560

STP2570

STP 2580

STP2590

STP 2600

STP2610

STP 2620

C

C

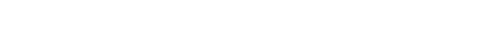

STP2630

FORMATS :

STP2650

STP2660

STP2670

STP 2680

-STP2690

STP 2700

200 FORMAT (' ' , 43X, 2I5, 3F 11.2)

STP2710

210 FORMAT ( $'-1,50 \mathrm{X}$, 'HEAD AND DRAWDOWN IN PUMPING WELLS' $/ 51 \mathrm{X}, 34\left({ }^{\prime}-1\right) / /$ STP2 2720 $148 X, ' I$ J WELL RADIUS HEAD DRAWDOWN' //)

STP2730

220 FORMAT (' ', 43X,2I5,' WELL IS DRY')

STP2740

240 FORMAT ('OMAXIMUM CHANGE IN HEAD FOR THIS TIME STEP $=$ ',F10.3/' ',5STP2750 $13('-1))$

250 FORMAT $(1 \mathrm{H} 1,47 \mathrm{X}$, 'MAXIMUM HEAD CHANGE FOR EACH ITERATION' / 48X,

STP2760

$\$ 38(1-1) / /)$

255 FORMAT (50X, I4, 6X, I4, 6X, F12.4)

260 FORMAT (' 1 ', 60X, 'HEAD MATRIX'/61X,11('-'))

STP2770

STP2780

STP2790

STP2800

270 FORMAT ('ODIMENSIONLESS TIME FOR THIS STEP RANGES FROM',G15.7,' TSTP2810 $\left.10^{\prime}, \mathrm{G} 15.7\right)$

STP2820

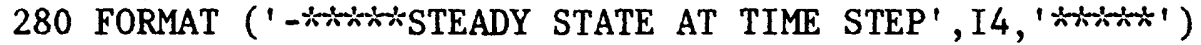

STP2830

290 FORMAT ( $1 \mathrm{H} 1,44 \mathrm{X}, 57\left({ }^{\prime}-1\right) / 45 \mathrm{X}$, ' $^{\prime}$ ', $14 \mathrm{X}$, 'TIME STEP NUMBER $=$ ' , I9, 14X, ' 'STP2840

$1 ' / 45 X, 57('-') / / 50 X, 29 H S I Z E$ OF TIME STEP IN SECONDS=,F14.2//55X, 'TOSTP2850

2TAL SIMULATION TIME IN SECONDS $=$ ', F14.2/80X, 8HMINUTES $=, F 14.2 / 82 \mathrm{X}, 6$ HSTP2860

3HOURS $=, \mathrm{F} 14.2 / 83 \mathrm{X}, 5 \mathrm{HDAYS}=, \mathrm{F} 14.2 / 82 \mathrm{X}$, 'YEARS $=$ ', $\mathrm{F} 14.2 / / / 45 \mathrm{X}$, 'DURATION STP2870

4OF CURRENT PUMPING PERIOD IN DAYS $=$ ',F14.2/82X, 'YEARS $=$ ', F14.2///56XSTP2880

5, ' ITERATION NUMBER=', I10/56X, 27( $\left.\left({ }^{\prime}-1\right) / /\right)$

300 FORMAT (8F10.4)

310 FORMAT (4G20.10)

1190 FORMAT (1HO)

END

STP2890

STP2900

STP29 10

STP2920

STP2930

SUBROUTINE SOLVE1(PHI , BE , G, TEMP, KEEP, PHE, STRT , T , S , QRE, WELL, TL , SL , DSIP 10 1EL , ETA , V , XI ,DELX, BET , DELY , ALF , SURI , TOP , NODE ID) $\quad$ SIP 20

C

$\begin{array}{llr}\text { SOLUTION BY THE STRONGLY IMPLICIT PROCEDURE } & \text { SIP } & 40 \\ & \text { SIP } & 60 \\ \text { SPECIFICATIONS: } & \text { SIP } & 70 \\ \text { COMMON /DPARAM/ RHO, B,D,F,H } & \text { SIP } & 80 \\ \text { COMMON /SARRAY/ TEST3(102),VF4(11),CHK(15), ITST(102), JTST(102) } & \text { SIP } & 90\end{array}$

COMMON /SPARAM/ WATER, CONVRT, EVAP, CHCK, PNCH, NUM, HEAD, CONTR, EROR, LESIP 100 1 AK, RECH , SIP , U, SS , TT , TMIN , ETDIST , QET , ERR, TMAX, CDLT , HMAX, YDIM, WIDTH , SIP 110 2NUMS , LSOR , ADI , DELT , SUM, SUMP , SUBS , STORE, TEST, ETQB , ETQD , FACTX, FACTY, SIP 120 3IERR, KOUNT, IF INAL, NUMT , KT , KP , NPER, KTH, ITMAX, IENGTH, NWEL , NW, DIML , DISIP 130 4MW , JNO1 , INO1 , R , P , PU, I , J , NODE , STDY , KPH, IQPNC

SIP 140 
Table 1.--Listing of computer program for Laramie County model--Continued

COMMON /ARSIZE/ IZ,JZ,IP, JP, IR, JR, IC , JC, IL , JL, IS , JS , IH, IMAX

$\$, I U, J U \quad$ SIP 160

COMMON /STREAM/ NRIV,NRPR,NODRV(20),NDR(20),NMRV(20),QRIV(3,20), SIP 170

$\mathrm{C}$

$\$ \operatorname{IRUP}(20), \operatorname{JRUP}(20), \operatorname{IRDN}(20), \operatorname{JRDN}(20)$

SIP 180

SIP 190

DIMENSION PHI (IZ,JZ), BE(IMAX), G(IMAX), TEMP(IMAX), KEEP(IZ,JZ), SIP 200 $1 \mathrm{PHE}(\mathrm{IZ}, \mathrm{JZ}), \operatorname{STRT}(\mathrm{IZ}, \mathrm{JZ}), \mathrm{T}(\mathrm{IZ}, \mathrm{JZ}), \mathrm{S}(\mathrm{IZ}, \mathrm{JZ}), \mathrm{QRE}$ (IZ,JZ), WELL(IZ,JSIP 210 2Z), TL(IZ,JZ), SL(IZ,JZ), DEL(IS,JS), ETA(IS, JS), V(IS,JS), XI (IS, SIP 220 3JS), DELX(JZ), BET(JZ), DELY(IZ), ALF(IZ), SURI (IZ,JZ),

C $\$$ TOP $(I C, J C)$, NODEID (IU, JU)

SIP 230

SIP 240

SIP 250

REAL *8PHI ,DBLE , RHOP(20), G, BE , TEMP , DABS , W, TEST2, DMAX1 , RHO , B , D , F , H , SIP 260 $1 \mathrm{~B} 1, \mathrm{E}, \mathrm{CH}, \mathrm{GH}, \mathrm{BH}, \mathrm{DH}, \mathrm{EH}, \mathrm{FH}, \mathrm{HH}, \mathrm{ALFA}, \mathrm{BETA}, \mathrm{GAHA}, \mathrm{RES}$

SIP 270

REAL $\div 4 \mathrm{KEEP}$

SIP 280

INTEGER R, P , PU, DIML , DIMW, CHK, WATER, CONVRT , EVAP , CHCK, PNCH, NUM, HEAD , SIP 290 ICONTR , LEAK, RECH, SIP , IORDER (21) , ADI , STDY

DATA KNTO/-1/

SIP 300

RETURN

SIP 310

SIP 320

C

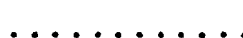

SIP 330

C

C

C

---COMPUTE AND PRINT ITERATION PARAMETERS--

SIP 340

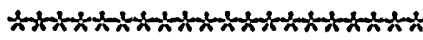

SIP 350

ENTRY ITER1

SIP 360

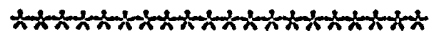

SIP 370

C

C

$\mathrm{C}$

---INITIALIZE ORDER OF ITERATION PARAMETERS (OR REPLACE WITH A

READ STATEMENT) ---

DATA IORDER/ $1,2,3,4,5,1,2,3,4,5,11 * 1 /$

SIP 380

I $2=$ INO1 -1

$\mathrm{J} 2=\mathrm{JN} 01-1$

L2 $=$ LENGTH $/ 2$

PL2=L2-1.

$W=0$.

$\mathrm{PI}=0$.

SIP 390

SIP 400

SIP 410

SIP 420

SIP 430

SIP 440

SIP 450

SIP 460

SIP 470

SIP 480

C --COMPUTE AVERAGE MAXIMUM PARAMETER FOR PROBLEM---

SIP 490

DO $10 \mathrm{I}=2$, INO1

SIP 500

DO $10 \mathrm{~J}=2, \mathrm{JNO} 1$

$\operatorname{SIP} 510$

IF $\left(\mathrm{T}(\mathrm{I}, \mathrm{J}) . \mathrm{EQ} .0_{.}\right)$GO TO 10

SIP 520

$\mathrm{PI}=\mathrm{PI}+1$.

SIP 530

$\mathrm{DX}=\mathrm{DELX}(\mathrm{J}) /$ WIDTH

SIP 540

$D Y=D E L Y(I) / Y D I M$

SIP 550

$W=W+1 .-A M I N 1(2 . * D X * D X /(1 .+F A C T Y * D X * D X /(F A C T X * D Y * D Y)), 2 . * D Y * D Y /(1 .+S I P 560$

1 FACTX $* D Y * D Y /(F A C T Y * D X * D X)))$

10 CONTINUE

$\mathrm{W}=\mathrm{W} / \mathrm{PI}$

SIP 570

SIP 580

SIP 590

C

C

---COMPUTE PARAMETERS IN GEOMETRIC SEQUENCE---

SIP 600

$\mathrm{PJ}=-1$.

SIP 610

SIP 620

DO $20 \quad I=1, L 2$

SIP 630

$\mathrm{PJ}=\mathrm{PJ}+1$.

c

$20 \operatorname{TEMP}(I)=1 .-(1 .-W) \approx(P J / P L 2)$

SIP 640

SIP 650

SIP 660

SIP 670

---ORDER SEQUENCE OF PARAMETERS---

DO $30 \mathrm{~J}=1$, LENGTH

SIP 680

$30 \operatorname{RHOP}(\mathrm{J})=\operatorname{TEMP}(\operatorname{IORDER}(\mathrm{J}))$

SIP 690 
Table 1.--Listing of computer program for Laramie County model--Continued

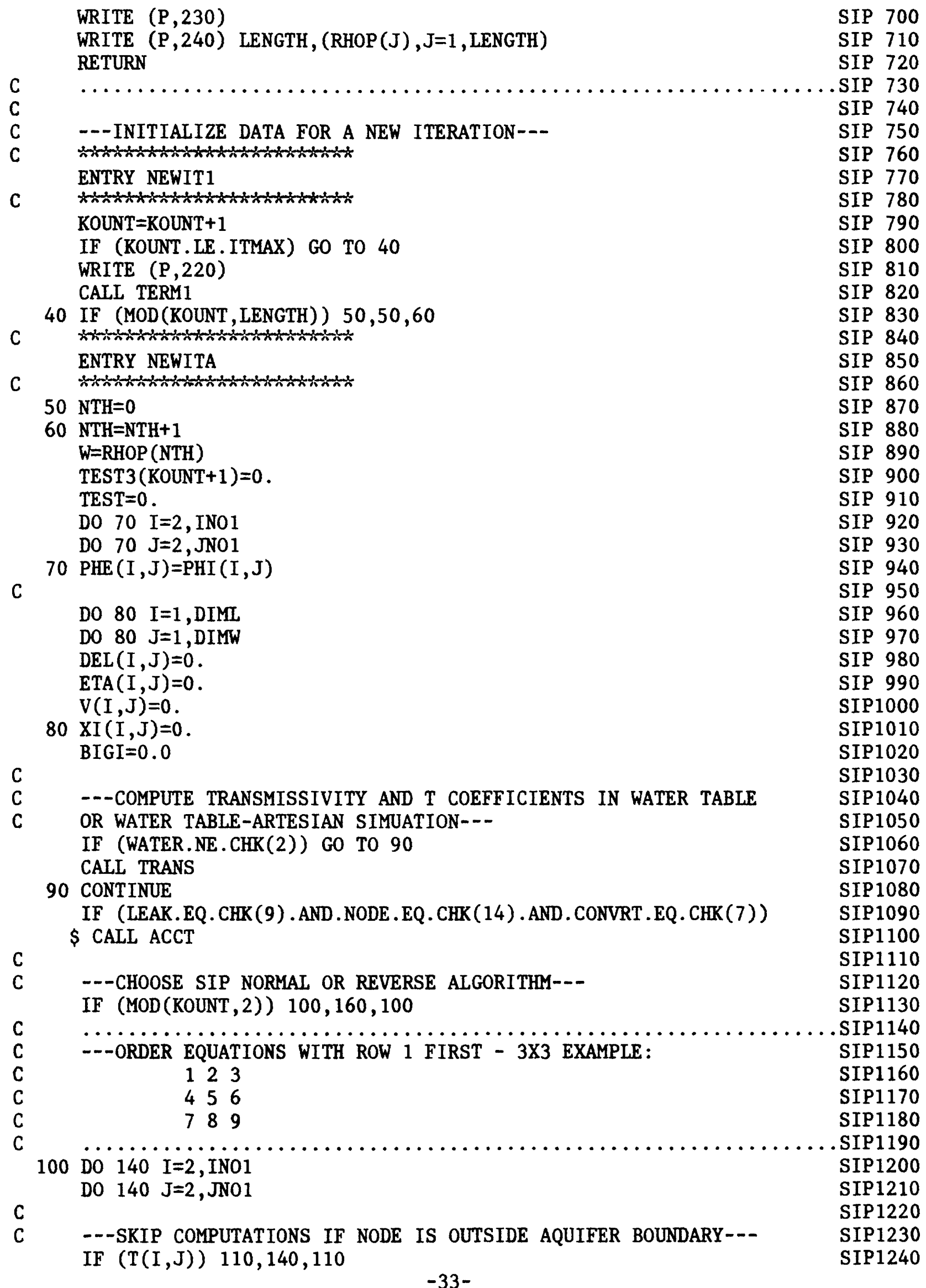


Table 1.--Listing of computer program for Laramie County model--Continued

$\mathrm{C}$

110 IF $(\mathrm{S}(\mathrm{I}, \mathrm{J})$.LT.0.) GO TO 120

C ---COMPUTE COEFFICIENTS---

IF (LEAK. NE.CHK(9).AND.CONVRT.NE.CHK(7)) GO TO 129

$\mathrm{U}=1.0$

IF (PHE $(I, J) \cdot \operatorname{LT} . \operatorname{TOP}(I, J)) \quad U=0.0$

129 CONTINUE

CALL COEF 1

GO TO 130

$120 \mathrm{RHO}=1 . \mathrm{D} 40$

C

C ---SIP 'NORMAL' ALGORITHM---

C ---FORWARD SUBSTITUTE, COMPUTING INTERMEDIATE VECTOR V---

$130 E=-B-D-F-H-R H O-T L(I, J) * U-E T Q B$

$\mathrm{CH}=\mathrm{DEL}(\mathrm{I}-1, \mathrm{~J}) \div \mathrm{B} /(1 .+W * \mathrm{DEL}(I-1, \mathrm{~J}))$

$\mathrm{GH}=\mathrm{ETA}(\mathrm{I}, \mathrm{J}-1) \star \mathrm{D} /(1 .+\mathrm{W} * \mathrm{ETA}(\mathrm{I}, \mathrm{J}-1))$

$\mathrm{BH}=\mathrm{B}-W^{2}=\mathrm{CH}$

$\mathrm{DH}=\mathrm{D}-\mathrm{W} \div \mathrm{GH}$

$\mathrm{EH}=\mathrm{E}+\mathrm{W} * \mathrm{CH}+W \div \mathrm{GH}$

$\mathrm{FH}=\mathrm{F}-\mathrm{W} * \mathrm{CH}$

$\mathrm{HH}=\mathrm{H}-\mathrm{W} * \mathrm{GH}$

ALFA $=\mathrm{BH}$

$\mathrm{BETA}=\mathrm{DH}$

GAMA $=E H-A L F A \div E T A(I-1, J)-B E T A * D E L(I, J-1)$

$\operatorname{DEL}(I, J)=F H /$ GAMA

$\operatorname{ETA}(I, J)=H H / G A M A$
$\operatorname{SIP} 1250$

SIP 1260

SIP 1270

SIP 1280

SIP1290

SIP 1300

SIP 1310

SIP1320

SIP 1330

$\operatorname{SIP} 1340$

SIP 1350

SIP 1360

SIP 1370

SIP 1380

SIP1390

SIP 1400

SIP1410

SIP 1420

SIP1430

SIP1440

SIP 1450

SIP 1460

SIP1470

SIP 1480

SIP 1490

SIP 1500

$\mathrm{RES}=-\mathrm{D} * \mathrm{PHI}(\mathrm{I}, \mathrm{J}-1)-\mathrm{F} * \mathrm{PHI}(\mathrm{I}, \mathrm{J}+1)-\mathrm{H} * \mathrm{PHI}(\mathrm{I}+1, \mathrm{~J})-\mathrm{B} * \mathrm{PHI}(\mathrm{I}-1, \mathrm{~J})-\mathrm{E} * \mathrm{PHI}(\mathrm{I}, \mathrm{JSIP} 1510$

1) $-\operatorname{RHO} \div \operatorname{KEEP}(I, J)-\operatorname{SL}(I, J)-Q R E(I, J)-\operatorname{WELL}(I, J)+\operatorname{ETQD}-\operatorname{SUBS}-\mathrm{TL}(I, J) * \operatorname{STRT}(\operatorname{SIP} 1520$

2I, J)

$V(I, J)=(R E S-A L F A * V(I-1, J)-B E T A * V(I, J-1)) /$ GAMA

IF (NODE.NE.CHK(14)) GO TO 138

IF (IABS (NODEID $(I, J))$.NE.99) GO TO 138

IF (KOUNT.NE.KNTO) WRITE $(P, 6900)$ KOUNT

SIP 1530

SIP 1540

SIP 1550

$\operatorname{SIP} 1560$

SIP1570

SIP 1580

KNTO $=$ KOUNT

$\operatorname{WRITE}(P, 6910) I, J, U, P H E(I, J), P H I(I, J), \operatorname{SL}(I, J), T L(I, J)$, WELL $(I, J)$,

$\$$ QRE $(I, J), R H O, E T Q D, E, B, D, F, H$

138 CONTINUE

6900 FORMAT (1HO,' ITERATION NO.' ,I4/2X,17('-'))

6910 FORMAT (2I4,F5.0, IP7E17.5 / (13X, 1P7E 17.5))

140 CONTINUE

C

C ---BACK SUBSTITUTE FOR VECTOR XI---

DO $150 \quad I=1, I 2$

I3 $=$ DIML $-I$

SIP1590

SIP 1600

SIP1610

SIP1620

SIP 1630

SIP 1640

SIP 1650

SIP 1660

SIP1670

SIP1680

DO $150 \mathrm{~J}=1, \mathrm{~J} 2$

SIP1690

$\mathrm{J} 3=\mathrm{DIMW}-\mathrm{J}$

IF (T(I3,J3).EQ.0.) GO TO 150

SIP 1700

SIP1710

$\mathrm{XI}(\mathrm{I} 3, \mathrm{~J} 3)=\mathrm{V}(\mathrm{I} 3, \mathrm{~J} 3)-\operatorname{DEL}(\mathrm{I} 3, \mathrm{~J} 3) * \mathrm{XI}(\mathrm{I} 3, \mathrm{~J} 3+1)-\mathrm{ETA}(\mathrm{I} 3, \mathrm{~J} 3) \div \mathrm{XI}(\mathrm{I} 3+1, \mathrm{~J} 3)$

C

C ---COMPARE MAGNITUDE OF CHANGE WITH CLOSURE CRITERIA---

SIP 1720

SIP 1730

SIP 1740

TCHK $=\mathrm{ABS}(\mathrm{XI}(\mathrm{I} 3, \mathrm{~J} 3))$

SIP1750

IF (TCHK.LT.BIGI) GO TO 145

$\mathrm{BIGI}=\mathrm{TCHK}$

SIP 1760

ITST $3=I 3$

SIP 1770

JTST $3=\mathrm{J} 3$ 
Table 1.--Listing of computer program for Laramie County model--Continued

$145 \operatorname{PHI}(\mathrm{I} 3, \mathrm{~J} 3)=\operatorname{PHI}(\mathrm{I} 3, \mathrm{~J} 3)+\mathrm{XI}(\mathrm{I} 3, \mathrm{~J} 3)$

150 CONTINUE

IF (BIGI.GT.ERR) TEST $=1$.

TEST $3($ KOUNT +1$)=$ BIGI

$\operatorname{ITST}($ KOUNT+1) $=$ ITST 3

JTST $($ KOUNT +1$)=$ JTST 3

RETURN

SIP 1800

SIP1810

SIP 1820

SIP 1830

SIP 1840

SIP1 850

C

$\ldots \ldots \ldots \ldots \ldots \ldots \ldots \ldots \ldots \ldots \ldots \ldots \ldots \ldots \ldots \ldots \ldots \ldots \ldots \ldots \ldots$

--ORDER EQUATIONS WITH THE LAST ROW FIRST - 3X3 EXAMPLE:

SIP 1860

SIP 1870

C

C

789

456

SIP 1880

$\mathrm{C}$

C

160 DO 200 II $=1, \mathrm{I} 2$

123

SIP 1890

SIP 1900

SIP1910

SIP 1920

.SIP1930

$I=D I M L-I I$

SIP 1940

SIP1950

DO $200 \mathrm{~J}=2$, JNO1

SIP1960

C

C

C

---SKIP COMPUTATIONS IF NODE IS OUTSIDE AQUIFER BOUNDARY---

SIP1970

IF $(T(I, J)) 170,200,170$

SIP 1980

SIP 1990

SIP 2000

SIP 2010

SIP 2020

---COMPUTE COEFFICIENTS---

IF (LEAK.NE.CHK(9).AND.CONVRT.NE.CHK(7)) GO TO 179

$\mathrm{U}=1.0$

IF $(P H E(I, J) \cdot \operatorname{LT} \cdot \operatorname{TOP}(I, J)) \quad U=0.0$

SIP 2030

SIP 2040

179 CONTINUE

CALL COEF 1

SIP2050

GO TO 190

SIP2060

SIP2070

$180 \mathrm{RHO}=1 . \mathrm{D} 40$

SIP2080

C

C ---SIP 'REVERSE' ALGORITHM---

C ---FORWARD SUBSTITUTE, COMPUTING INTERMEDIATE VECTOR V---

$190 \mathrm{E}=-\mathrm{B}-\mathrm{D}-\mathrm{F}-\mathrm{H}-\mathrm{RHO}-\mathrm{TL}(\mathrm{I}, \mathrm{J}) * \mathrm{U}-\mathrm{ETQB}$

$\mathrm{CH}=\operatorname{DEL}(\mathrm{I}+1, \mathrm{~J}) \div \mathrm{H} /\left(1 .+\mathrm{W}^{*} \mathrm{DEL}(\mathrm{I}+1, \mathrm{~J})\right)$

$\mathrm{GH}=\mathrm{ETA}(\mathrm{I}, \mathrm{J}-1) * \mathrm{D} /(1 .+\mathrm{W} * \mathrm{ETA}(\mathrm{I}, \mathrm{J}-1))$

$\mathrm{BH}=\mathrm{H}-\mathrm{W}^{2} \mathrm{CH}$

$\mathrm{DH}=\mathrm{D}-\mathrm{W} * \mathrm{GH}$

$\mathrm{EH}=\mathrm{E}+W * \mathrm{CH}+W \% \mathrm{GH}$

$\mathrm{FH}=\mathrm{F}-\mathrm{W} \div \mathrm{CH}$

$\mathrm{HH}=\mathrm{B}-\mathrm{W} * \mathrm{GH}$

$\mathrm{ALFA}=\mathrm{BH}$

$\mathrm{BETA}=\mathrm{DH}$

GAMA $=\mathrm{EH}-\mathrm{ALFA} * \mathrm{ETA}(\mathrm{I}+1, \mathrm{~J})-\mathrm{BETA} * \mathrm{DEL}(\mathrm{I}, \mathrm{J}-1)$

$\operatorname{DEL}(\mathrm{I}, \mathrm{J})=\mathrm{FH} /$ GAMA

$\operatorname{ETA}(I, J)=$ HH $/$ GAMA

SIP2090

SIP2100

SIP2110

SIP2120

SIP2130

SIP2140

SIP2150

SIP2160

SIP2170

SIP2180

SIP2190

SIP2200

SIP2210

SIP2220

SIP 2230

SIP2240

SIP2250

RES $=-D * P H I(I, J-1)-F^{*} \div$ PHI $(I, J+1)-H * P H I(I+1, J)-B * P H I(I-1, J)-E * P H I(I, J S I P 2260$

1) $-\operatorname{RHO} * \operatorname{KEEP}(I, J)-\operatorname{SL}(I, J)-Q R E(I, J)-W E L L(I, J)+E T Q D-S U B S-T L(I, J) * S T R T(S I P 2270$ 2I, J)

$V(I, J)=(\operatorname{RES}-A \operatorname{ALA} * \mathrm{~V}(\mathrm{I}+1, \mathrm{~J})-\operatorname{BETA} * \mathrm{~V}(\mathrm{I}, \mathrm{J}-1)) /$ GAMA

SIP2280

SIP2290

IF (NODE.NE.CHK(14)) GO TO 198

SIP2300

IF (IABS (NODEID $(I, J)$ ).NE.99) GO TO 198

SIP2310

IF (KOUNT.NE. KNTO) WRITE $(P, 6900)$ KOUNT

SIP2320

KNTO=KOUNT

SIP2330

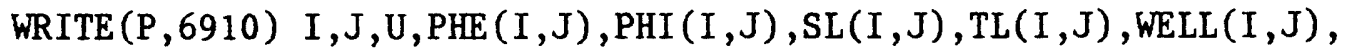
$\operatorname{SIP} 2340$ 
Table 1.--Listing of computer program for Laramie County model--Continued

\$ QRE (I , J) , RHO ,ETQD , E , B , D , F , H $\quad$ SIP2350

198 CONTINUE

SIP2360

200 CONTINUE

SIP2370

C

C ---BACK SUBSTITUTE FOR VECTOR XI---

SIP2380

DO 210 I3 $=2$, INO1

SIP2390

DO $210 \mathrm{~J}=1, \mathrm{~J} 2$

SIP2400

$\mathrm{J} 3=\mathrm{DIMW}-\mathrm{J}$

SIP2410

IF (T (I3,J3).EQ.0.) GO TO 210

SIP2420

$\mathrm{XI}(\mathrm{I} 3, \mathrm{~J} 3)=\mathrm{V}(\mathrm{I} 3, \mathrm{~J} 3)-\operatorname{DEL}(\mathrm{I} 3, \mathrm{~J} 3) * \mathrm{XI}(\mathrm{I} 3, \mathrm{~J} 3+1)-\operatorname{ETA}(\mathrm{I} 3, \mathrm{~J} 3) * \mathrm{XI}(\mathrm{I} 3-1, \mathrm{~J} 3)$

SIP2430

$\mathrm{C}$

C ---COMPARE MAGNITUDE OF CHANGE WITH CLOSURE CRITERIA---

SIP2440

SIP2450

TCHK=ABS $(X I(I 3, J 3))$

SIP2460

IF (TCHK.LT.BIGI) GO TO 205

SIP2470

$\mathrm{BIGI}=\mathrm{TCHK}$

SIP2480

SIP2490

ITST $3=\mathrm{I} 3$

SIP2500

JTST $3=\mathrm{J} 3$

SIP25 10

$205 \operatorname{PHI}(\mathrm{I} 3, \mathrm{~J} 3)=\operatorname{PHI}(\mathrm{I} 3, \mathrm{~J} 3)+\mathrm{XI}(\mathrm{I} 3, \mathrm{~J} 3)$

SIP2520

210 CONTINUE

SIP2530

IF (BIGI.GT.ERR) TEST $=1$.

SIP2540

TEST $3($ KOUNT +1$)=$ BIGI

SIP2550

ITST $($ KOUNT +1$)=$ ITST 3

SIP2560

$\operatorname{JTST}($ KOUNT +1$)=$ JTST 3

SIP2570

RETURN

SIP2580

SIP2590

c

C

$\ldots \ldots \ldots \ldots \ldots \ldots \ldots \ldots \ldots$

SIP2600

---FORMATS---

SIP2610

SIP2620

SIP2630

SIP2640

SIP2650

220 FORMAT ('OEXCEEDED PERMITTED NUMBER OF ITERATIONS' $/$ ' ',39(' $*$ ')) SIP2660

230 FORMAT (' - ', 44X, 'SOLUTION BY THE STRONGLY IMPLICIT PROCEDURE'/45X,SIP2670 $\left.143\left('{ }^{\prime}\right)\right)$

SIP2680

240 FORMATT $(/ / / 1 \mathrm{HO}$, I5,22H ITERATION PARAMETERS : 6D15.7/(/28X,6D15.7/))SIP2690 END

SIP2 700

SUBROUTINE COEF (PHI , KEEP , PHE, STRT , SURI , T, TR, TC , S , WELL , TL , SL , PERM , BCOF 10 10TTOM, SY , RATE, RIVER , M, TOP , GRND, DELX, DELY, NODEID , QBND)

$\mathrm{C}$

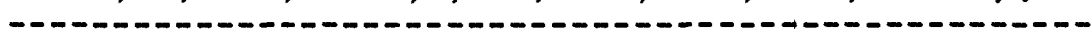

COF 30

COMPUTE COEFFICIENTS

COF 40

$\mathrm{C}$

$\mathrm{C}$

C

SPECIFICATIONS :

COMMON /DPARAM/ RHO,B,D,F,H

COF 50

COF 60

COMMON /SARRAY/ TEST3(102), VF4(11), CHK(15), ITST(102), JTST(102) COF 90

COMMON /SPARAM/ WATER, CONVRT, EVAP, CHCK, PNCH, NUM, HEAD, CONTR, EROR, LECOF 100

1 AK, RECH , SIP , U , SS , TT , TMIN , ETDIST , QET , ERR , TMAX, CDLT , HMAX, YDIM, WIDTH , COF 110

2NUMS , LSOR, ADI , DELT , SUM, SUMP , SUBS , STORE , TEST , ETQB , ETQD , FACTX, FACTY , COF 120

3IERR , KOUNT , IF INAL , NUMT , KT , KP , NPER , KTH , ITMAX, LENGTH , NWEL , NW , DIML , DICOF 130

4MW, JNO1, INO1, R , P , PU, I , J , NODE , STDY , KPH, IQPNC

COMMON /ARSIZE/ IZ, JZ, IP , JP, IR, JR, IC , JC, IL , JL, IS , JS , IH , IMAX

COF 140 $\$, I U, J$

COF 150

COF 160

$\mathrm{C}$

DIMENSION PHI (IZ,JZ), KEEP(IZ,JZ), PHE(IZ,JZ), STRT(IZ,JZ), SURI(ICOF 180 
Table 1.--Listing of computer program for Laramie County model--Continued

C

C

C

C

C

C

C

C

C

C

C

C

C

C
$12, \mathrm{JZ}), \mathrm{T}(\mathrm{IZ}, \mathrm{JZ}), \mathrm{TR}(\mathrm{IZ}, \mathrm{JZ}), \mathrm{TC}(\mathrm{IZ}, \mathrm{JZ}), \mathrm{S}(\mathrm{IZ}, \mathrm{JZ})$, WELL(IZ,JZ), TL(ICOF 190 $2 Z, J Z$ ), SL (IZ,JZ), PERM(IP,JP), BOTTOM(IP, JP), SY (IP, JP), RATE (IR, JCOF 200 3R), RIVER(IR, JR), M(IR, JR), TOP (IC,JC), GRND (IL ,JL), DELX(JZ), DELCOF 210 4Y (IZ) , NODEID (IU, JU) , QBND (IU, JU) $\quad$ COF 220 COMMON /STREAM/ NRIV, NRPR, NODRV (20), NDR(20), NMRV (20),QRIV $(3,20), \quad$ COF 230 $\$ \operatorname{IRUP}(20), \operatorname{JRUP}(20), \operatorname{IRDN}(20), \operatorname{JRDN}(20)$

COF 240

COF 250

COF 260

COF 270

REAL *8PHI , DBLE, RHO , B , D , F, H

INTEGER R,P , PU, DIML , DIMW , CHK, WATER, CONVRT , EVAP , CHCK, PNCH, NUM, HEAD , COF 280 1CONTR , LEAK, RECH , SIP , ADI , STDY

DATA PIE/3.141593/

RETURN

COF 290

COF 300

COF 310

COF 320

COF 330

---COMPUTE COEFFICIENTS FOR TRANSIENT PART OF LEAKAGE TERM---

COF 340

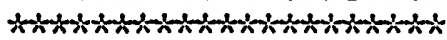

ENTRY CLAY

COF 350

COF 360

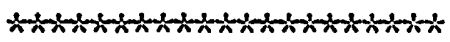

COF 370

TMIN $=1 . E 40$

$\mathrm{TT}=0.0$

PRATE $=0$.

DO $50 \mathrm{I}=1$, DIML

DO $50 \mathrm{~J}=1$, DIMW

COF 380

COF 390

COF 400

COF 410

COF 420

COF 430

COF 440

COF 450

COF 460

COF 470

COF 480

COF 490

COF 500

COF 510

COF 520

COF 530

COF 540

HEAD BOUNDARY---
COF 550

IF $(\operatorname{RATE}(I, J) \cdot L E .0 \ldots O R \cdot T(I, J) \cdot E Q \cdot 0 \ldots O R \cdot M(I, J) \cdot E Q \cdot 0 \ldots O R . S(I, J) \cdot L T .0 C O F 560$
1.) GO TO 50

COF 580

COF 590

COF 600

COF 610

COF 620

COF 630

COF 640

COF 650

COF 660

PPT $=P I E * P I E * D I M T$

COF 670

COF 680

COF 690

IF (DIMT.LT . 1.0E-03) PPT $=1.0 /$ DIMT

$\mathrm{CC}=(2.3-\mathrm{PPT}) /(2 . \approx \mathrm{PPT})$

COF 700

COF 710

COF 720

SUMN $=0.0$

DO $20 \mathrm{~K}=1,200$ 
Table 1.--Listing of computer program for Laramie County model--Continued

POWER $=K * K * P P T$

IF (POWER.LE.150.) GO TO 10

COF 740

POWER $=150$

10 PEX=EXP (-POWER)

SUMN=SUMN+PEX

IF (PEX.GT.0.00009) GO TO 20

COF 750

COF 760

COF 770

IF (K.GT.CC) GO TO 30

C

20 CONTINUE

C

30 DENOM $=1.0$

IF (DIMT.LT . 1.OE-03) DENOM=SQRT(PIE*DIMT)

COF 780

COF 790

COF 800

COF 810

COF 820

COF 830

COF 840

COF 850

COF 860

c

---HEAD VALUES ARE NOT INCLUDED IN COMPUTATION OF Q FACTOR SINCE

COF 870

LEAKAGE IS CONSIDERED IMPLICITLY---

$40 Q 1=\operatorname{RATE}(I, J) /(M(I, J) * D E N O M)$

$\mathrm{TL}(I, J)=Q 1+2 . * Q 1 *$ SUMN

COF 880

COF 890

PRATE $=\operatorname{RATE}(I, J) * M(I, J)$

COF 900

50 CONTINUE

COF 910

TMIN $=$ TMIN $* 3.0$

COF 920

$\mathrm{TT}=\mathrm{TT} * 3.0$

COF 930

RETURN

COF 940

COF 950

C

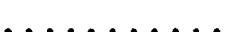

COF 960

C

C

C

C

---COMPUTE TRANSMISSIVITY IN WT OR WT-ARTESIAN CONVERSION PROBLEM-CC

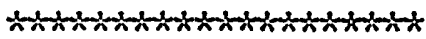

COF 970

ENTRY TRANS

COF 990

COF 1000

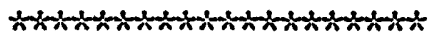

COF 1010

DO $60 \mathrm{I}=1$, DIML

COF 1020

DO $60 \mathrm{~J}=1$, DIMW

COF 1030

IF (PERM (I,J).EQ.0.) GO TO 60

COF 1040

$\mathrm{HED}=\operatorname{PHI}(\mathrm{I}, \mathrm{J})$

COF 1050

IF (CONVRT.EQ.CHK (7) . AND .TOP (I ,J) . GT . 0.0) HED=AMIN1 (SNGL (PHI (I,J)) COF1060

$\$, \operatorname{TOP}(I, J))$

$T(I, J)=\operatorname{PERM}(I, J) *($ HED $-B O T T O M(I, J))$

COF 1070

IF $(T(I, J) . G E .0$.$) GO TO 60$

C

IF (WELL $(I, J) . L T .0$.$) GO TO 70$

IF (WELL $(I, J) . G E .0 .0)$ GO TO 61

IF (NODE.EQ.CHK(14).AND.NODEID $(I, J) . I T .0)$ GO TO 61

GO TO 70

$\mathrm{C}$

61 CONTINUE

C

---THE FOLLOWING STATEMENTS APPLY WHEN NODES (EXCEPT WELL NODES)

GO DRY---

$\operatorname{PERM}(I, J)=0$.

COF 1080

COF 1090

COF 1100

COF 1110

COF 1120

COF 1130

COF 1140

COF 1150

$T(I, J)=0.0$

$\operatorname{TR}(\mathrm{I}, \mathrm{J}-1)=0$.

$\operatorname{TR}(I, J)=0$.

$\mathrm{TC}(I-1, J)=0$.

$\operatorname{TC}(I, J)=0$.

$\operatorname{PHI}(I, J)=\operatorname{SURI}(I, J)$

WRITE $(P, 240) I, J$

COF 1160

COF 1170

COF 1180

COF 1190

COF 1200

COF 1210

COF 1220

COF 1230

COF 1240

COF 1250

60 CONTINUE

COF 1260

IF (KT.EQ.0) RETURN

COF 1270

GO TO 90

COF 1280 
Table 1.--Listing of computer program for Laramie County model--Continued

C

C

70 WRITE $(P, 210)$ I , J, KOUNT

WRITE $(P, 220)$

IERR $=1$

CALL DRDN

DO $80 \mathrm{I}=2$, INO1

DO $80 \mathrm{~J}=2$, JNO1

$80 \operatorname{PHI}(I, J)=\operatorname{KEEP}(I, J)$

SUM=SUM-DELT

SUMP $=$ SUMP $-D E L T$

$\mathrm{KT}=\mathrm{KT}-1$

IF (KT.EQ.0) STOP

CALL PUNCH

IF (MOD (KT, KTH) .EQ.0) STOP

WRITE $(P, 230)$ KT, SUM

CALL DRDN

IF (CHCK.EQ.CHK(5)) CALL CWRITE

STOP

C

C

---COMPUTE T COEFFICIENTS---

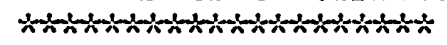

ENTRY TCOF

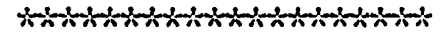

0 DO $110 \mathrm{I}=1$, INO1

D0 $110 \mathrm{~J}=1$, JNO1

IF $(T(I, J) . L E .0 .0)$ GO TO 110

IF $(T(I, J+1) . L E .0 .0)$ GO TO 100

$\mathrm{TR}(\mathrm{I}, \mathrm{J})=(2 . * \mathrm{~T}(\mathrm{I}, \mathrm{J}+1) * \mathrm{~T}(\mathrm{I}, \mathrm{J})) /(\mathrm{T}(\mathrm{I}, \mathrm{J}) * \operatorname{DELX}(\mathrm{J}+1)+\mathrm{T}(\mathrm{I}, \mathrm{J}+1) * \operatorname{DELX}(\mathrm{J})) * \mathrm{FCOF} 1570$ 1ACTX

100 IF (T $(\mathrm{I}+1, \mathrm{~J}) . \mathrm{LE} .0 .0)$ GO TO 110

IACTY

110 CONTINUE

RETURN

$\mathrm{C}$

C

$\mathrm{C}$

C

C

C

C

C .......

---COMPUTE COEFFICIENTS---

A

ENTRY COEF1

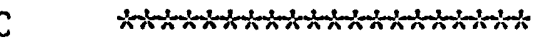

$\mathrm{D}=\mathrm{TR}(\mathrm{I}, \mathrm{J}-1) / \mathrm{DELX}(\mathrm{J})$

$F=\operatorname{TR}(I, J) / D E L X(J)$

$\mathrm{B}=\mathrm{TC}(\mathrm{I}-1, \mathrm{~J}) / \mathrm{DELY}(\mathrm{I})$

$\mathrm{H}=\mathrm{TC}(\mathrm{I}, \mathrm{J}) / \mathrm{DELY}(\mathrm{I})$

IF (EVAP.NE.CHK(6)) GO TO 120

---COMPUTE EXPLICIT AND IMPLICIT PARTS OF ET RATE---

$\mathrm{ETQB}=0$.

$\mathrm{ETQD}=0.0$

IF (PHE $(I, J)$.LE.GRND $(I, J)$-ETDIST) GO TO 120

ETQB $=Q E T / E T D I S T$

$E T Q D=E T Q B *(E T D I S T-G R N D(I, J))$
COF 1290

COF 1300

COF 1310

COF 1320

COF 1330

COF 1340

COF 1350

COF 1360

COF 1370

COF 1380

COF 1390

COF 1400

COF 1410

COF 1420

COF 1430

COF 1440

COF 1450

COF 1460

COF 1470

COF 1480

COF 1490

COF 1500

COF 1510

COF 1520

COF 1530

COF 1540

COF 1550

COF 1560

COF 1580

COF 1590

COF 1610

COF 1620

COF 1630

. COF 1640

COF 1650

COF 1660

COF 1670

COF 1680

COF 1690

COF 1700

COF 1710

COF 1720

COF 1730

COF 1740

COF 1750

COF 1760

COF 1770

COF 1780

COF 1790

COF 1800

COF 1810

COF 1820

COF 1830 
Table 1.--Listing of computer program for Laramie County model--Continued

120 IF (CONVRT.EQ.CHK(7)) GO TO 130

$\mathrm{RHO}=\mathrm{S}(\mathrm{I}, \mathrm{J}) / \mathrm{DELT}$

IF (WATER.EQ. CHK(2)) RHO=SY (I,J)/DELT

RETURN

C

C ---COMPUTE STORAGE COEFFICIENT FOR CONVERSION PROBLEM---

130 SUBS $=0.0$

IF $(\operatorname{KEEP}(I, J) . G E . T O P(I, J)$.AND.PHE $(I, J) . G E . T O P(I, J))$ GO TO 170

IF $(\operatorname{KEEP}(I, J) \cdot \operatorname{LT} \cdot \operatorname{TOP}(\mathrm{I}, \mathrm{J}) \cdot \operatorname{AND} \cdot \operatorname{PHE}(\mathrm{I}, \mathrm{J}) \cdot \mathrm{LT} . \mathrm{TOP}(\mathrm{I}, \mathrm{J}))$ GO TO 160

IF $(\operatorname{KEEP}(I, J)-P H E(I, J)) 140,150,150$

$140 \operatorname{SUBS}=(\operatorname{SY}(I, J)-S(I, J)) / \operatorname{DELT} *(\operatorname{KEEP}(I, J)-\operatorname{TOP}(I, J))$

GO TO 170

$150 \operatorname{SUBS}=(\mathrm{S}(\mathrm{I}, \mathrm{J})-\mathrm{SY}(\mathrm{I}, \mathrm{J})) / \operatorname{DELT} *(\operatorname{KEEP}(\mathrm{I}, \mathrm{J})-\mathrm{TOP}(\mathrm{I}, \mathrm{J}))$

COF 1840

COF 1850

COF 1860

COF 1870

COF 1880

COF 1890

COF 1900

COF 1910

COF 1920

COF 1930

COF 1940

COF 1950

COF 1960

COF 1970

COF 1980

GO TO 180

COF 1990

$170 \mathrm{RHO}=\mathrm{S}(\mathrm{I}, \mathrm{J}) / \mathrm{DELT}$

C

180 IF (LEAK.NE.CHK(9)) RETURN

COF 2000

COF 2010

COF 2020

COF 2030

IF (RATE $(I, J) . E Q .0$..OR.M(I, J).EQ.0.) GO TO 200

COF 2040

IF (NODE.NE.CHK(14).OR.NRPR.EQ.0) GO TO 182

DO $181 \mathrm{NR}=1$, NRPR

COF 2050

IF (IABS(NODEID $(I, J)$ ).EQ.NDR(NR)) GO TO 200

181 CONTINUE

182 CONTINUE

COF 2060

COF 2070

COF 2080

$\operatorname{HED} 1=\operatorname{AMAX} 1(\operatorname{STRT}(I, J), \operatorname{TOP}(I, J))$

COF 2090

$\mathrm{U}=1$.

HED2 $=0$.

IF (PHE $(I, J) . G E . T O P(I, J))$ GO TO 190

COF 2100

COF 2110

COF 2120

$\operatorname{HED} 2=\mathrm{TOP}(\mathrm{I}, \mathrm{J})$

COF 2130

$\mathrm{U}=0$.

COF 2140

$190 \operatorname{SL}(I, J)=\operatorname{RATE}(I, J) / M(I, J) *(\operatorname{RIVER}(I, J)-H E D 1)+T L(I, J) *(H E D 1-H E D 2-S T R T C O F 2150$

$1(\mathrm{I}, \mathrm{J}))$

C 200 RETURN

COF 2160

COF 2170

COF 2180

---STREAMFLOW ACCOUNTING PROCEDURE---

COF 2190

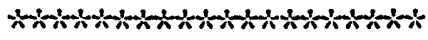

COF 2200

ENTRY ACCT

C

COF 2210

IF (NRPR.EQ.0) RETURN

COF 2220

COF 2230

DO $500 \mathrm{NV}=1$, NRPR

COF 2240

$\mathrm{NR}=\mathrm{NRPR}-\mathrm{NV}+1$

COF 2250

$I=I R U P(N R)$

COF 2260

$\mathrm{J}=\mathrm{JRUP}(\mathrm{NR})$

COF 2270

IF (IRDN(NR).NE.I.AND.JRDN(NR).NE.J) GO TO 301

COF 2280

$\mathrm{QRIV}(2, \mathrm{NR})=\mathrm{QRIV}(1, \mathrm{NR})$

COF 2290

GO TO 500

COF 2300

301 CONTINUE

COF 2310

$\mathrm{QRX}=\mathrm{QRIV}(1, \mathrm{NR})$

COF 2320

$\mathrm{ND}=\mathrm{NDR}(\mathrm{NR})$

COF 2330

300 IF (RATE (I,J).EQ.0.0.OR.M(I,J).EQ.0.0) GO TO 3900

COF 2340

C

---CALCULATE LEAKAGE TO/FROM STREAM AND RESULTAN'

STREAM DISCHARGECOF 2350

C IN CFS---

COF 2360

FACT $=\operatorname{RATE}(\mathrm{I}, \mathrm{J}) / \mathrm{M}(\mathrm{I}, \mathrm{J})$

COF 2370

$\operatorname{TL}(I, J)=F A C T$

COF 2380 
Table 1.--Listing of computer program for Laramie County model--Continued

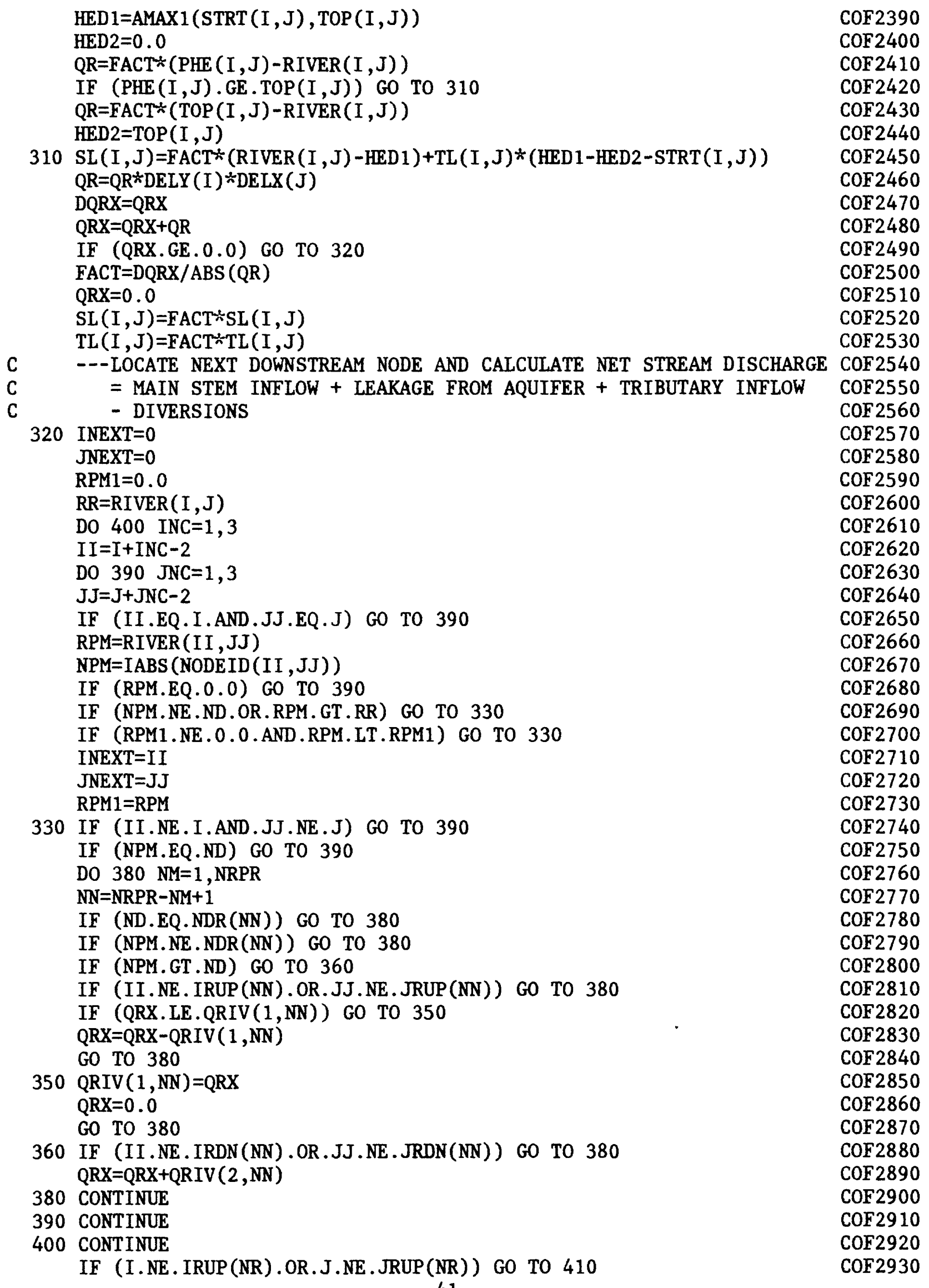


Table 1.--Listing of computer program for Laramie County model--Continued

$\mathrm{QRIV}(3, \mathrm{NR})=\mathrm{QRX}$

COF 2940

GO TO 430

COF 2950

COF 2960

410 IF (I.NE.IRDN(NR).OR.J.NE.JRDN(NR)) GO TO 420

COF 2970

$\mathrm{QRIV}(2, \mathrm{NR})=\mathrm{QRX}$

IF (NODEID $(I, J) . G T .0) \quad Q B N D(I, J)=Q R X$

COF 2980

GO TO 500

COF 2990

420 IF (NODEID $(I, J) . G T .0 .0) \quad Q B N D(I, J)=Q R X$

COF 3000

430 IF (INEXT. EQ.0.OR. JNEXT. EQ. 0) GO TO 440

COF 3010

$\mathrm{I}=\mathrm{INEXT}$

COF 3020

$\mathrm{J}=\mathrm{JNEXT}$

COF 3030

GO TO 300

COF 3040

$440 \operatorname{WRITE}(\mathrm{P}, 3920)$ NR, ND, I , J

COF 3050

$\mathrm{QRIV}(2, \mathrm{NR})=\mathrm{QRX}$

COF3060

$\operatorname{IRDN}(\mathrm{NR})=\mathrm{I}$

COF 3070

$\operatorname{JRDN}(\mathrm{NR})=\mathrm{J}$

COF 3080

500 CONTINUE

COF 3090

RETURN

COF 3100

3900 WRITE (P, 3910) NR,ND, I ,J

COF 3110

3910 FORMAT $\left(1 H 1,5 X,{ }^{\prime} K{ }^{\prime}\right.$ OR $M$ IS ZERO AT DESIGNATED STREAM NODE, STREAM COF 3120

\$NO. $={ }^{\prime}, \mathrm{I} 4,5 \mathrm{X},{ }^{\prime} \mathrm{NODEID}={ }^{\prime}, \mathrm{I} 4,5 \mathrm{X},{ }^{\prime} \mathrm{I}={ }^{\prime}, \mathrm{I} 4,5 \mathrm{X},{ }^{\prime} \mathrm{J}={ }^{\prime}, \mathrm{I} 4$ )

COF 3130

3920 FORMAT ( 1 HO, 5X,' INSUFFICIENT NUMBER OF STREAM NODES ENCOUNTERED FORCOF 3140

$\$$ STREAM NO.' $, \mathrm{I} 3,3 \mathrm{X},{ }^{\prime}$ NODEID $={ }^{\prime}, \mathrm{I} 3,3 \mathrm{X},,^{\prime} \mathrm{I}={ }^{\prime}, \mathrm{I} 3,3 \mathrm{X},{ }^{\prime} \mathrm{J}={ }^{\prime}, \mathrm{I} 3 / /$ ) COF3150 STOP

COF 3160

C

- -FORMATS---

COF 3170

COF3180

COF 3190

COF 3200

COF 3210

C

210 FORMAT (' -

12X,'AT ITERATION NO.' ', I3)

220 FORMAT (' 1 ', 50X, 'DRAWDOWN WHEN WELL WENT DRY')

COF 3230

COF 3240

230 FORMAT (' 1 ', 32X, 'DRAWDOWN FOR TIME STEP', I3,' ; SIMULATION TIME =',COF3250

$11 \mathrm{PE} 15.7, '$ SECONDS')

COF 3260

240 FORMAT ('-',20('*'),' NODE ',I4,',',I4,' GOES DRY ',20('*')) COF3270

END

COF 3280

SUBROUTINE CHECKI (PHI , KEEP , PHE , STRT , T , TR, TC , S , QRE , WELL , TL , PERM , BOTCHK 10 ITOM, SY, RATE , RIVER, M, TOP, GRND, DELX, DELY, NODE ID) $\quad$ CHK 20

C

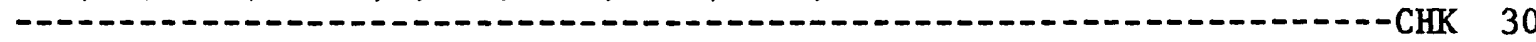

$\begin{array}{lr}\text { COMPUTE A MASS BALANCE } & \text { CHK } 40\end{array}$

СОИРИТ А МАSS ВАLАКСЕ

SPECIFICATIONS :

CHK 60

CHK 70

COMMON /SARRAY/ TEST3(102),VF4(11), CHK(15), ITST(102),JTST(102) CHK 80

COMMON /SPARAM/ WATER, CONVRT, EVAP, CHCK, PNCH, NUM, HEAD, CONTR, EROR, LECHK 90

1 AK , RECH , SIP , U , SS , TT , TMIN , ETDIST, QET , ERR, TMAX, CDLT , HMAX, YDIM, WIDTH , CHK 100

2NUMS , LSOR , ADI ,DELT , SUM , SUMP , SUBS , STORE , TEST , ETQB , ETQD , FACTX , FACTY , CHK 110

3IERR, KOUNT , IFINAL , NUMT , KT , KP , NPER , KTH, ITMAX , LENGTH , NWEL , NW , DIML , DICHK 120

4MW , JNO1, INO I , R , P , PU , I , J , NODE , STDY , KPH, IQPNC

CHK 130

COMMON /CK/ ETFLXT, STORT, QRET, CHST, CHDT, FLUXT, PUMPT, CFLUXT, FLXNTT CHK 140

COMMON /ARSIZE/ IZ , JZ, IP , JP , IR, JR, IC , JC, IL , JL, IS , JS , IH, IMAX

CHK 150

$\$, I U, \pi$

COMMON /NDID/ NOD(100), NMBR

CHK 160

CHK 170

C

DIMENSION PHI $(I Z, J Z), \operatorname{KEEP}(I Z, J Z), \operatorname{PHE}(I Z, J Z), \operatorname{STRT}(I Z, J Z), T(I Z, J C H K 190$ 
Table 1.--Listing of computer program for Laramie County model--Continued

1Z), TR(IZ,JZ), TC(IZ,JZ), S(IZ,JZ), QRE(IZ,JZ), WELL(IZ,JZ), TL (IZCHK 200 $2, \mathrm{JZ})$, PERM(IZ,JZ), BOTTOM(IP,JP), SY (IP, JP), RATE (IR, JR), RIVER (IRCHK 210 $3, J R), M(I R, J R), \operatorname{TOP}(I C, J C), \operatorname{GRND}(I L, J L), \operatorname{DELX}(J Z), \operatorname{DELY}(\mathrm{IZ}) \quad$ CHK 220 $4, \operatorname{NODEID}(\mathrm{IU}, \mathrm{JU})$

C DIMENSION XPT $(6)$, QFLX $(6,100)$

CHK 230

CHK 240

CHK 250

REAL *8PHI, DBLE

CHK 260

REAL *4KEEP,M

CHK 270

INTEGER R,P, PU, DIML , DIMW , CHK, WATER, CONVRT , EVAP , CHCK, PNCH, NUM, HEAD , CHK 280

1CONTR, LEAK, RECH , SIP , ADI , STDY

CHK 290

RETURN

CHK 300

C

C

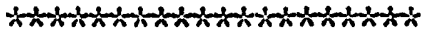

ENTRY CHECK

C

C ---INITIALIZE VARIABLES---

PUMP $=0$.

$\mathrm{STOR}=0$.

FLUXS $=0.0$

$\mathrm{CHD} 1=0.0$

$\mathrm{CHD} 2=0.0$

QREFLX $=0$.

CFLUX $=0$.

FLUX $=0$.

ETFLUX $=0$.

CHK 310

FLXN $=0.0$

IF (STDY.NE.CHK(15)) GO TO 400

CHK 320

CHK 330

CHK 340

IF (KT.NE.0) GO TO 400

CHK 350

CHK 360

CHK 370

CHK 380

CHK 390

CHK 400

CHK 410

CHK 420

CHK 430

CHK 440

CHK 450

DO 410 I=1, DIML

CHK 460

DO $410 \mathrm{~J}=1$, DIMW

CHK 470

CHK 480

$410 \operatorname{KEEP}(I, J)=\operatorname{STRT}(I, J)$

CHK 490

400 CONTINUE

CHK 500

CHK 510

C

CHK 580

CHK 590

CHK 600

IF (KPH.EQ.0) GO TO 311

IF (MOD(KP, KPH).NE.0) GO TO 320

CHK 610

CHK 620

311 IF (MOD(KT,KTH).EQ.0.OR. IFINAL.NE.0) WRITE(P,3600) KT CHK 630

3600 FORMAT(1H1,39X,'FLOW RATES (L $\left.L^{-4} 3 / T\right)$ AT SELECTED NODES AT TIME STEPCHK 640

$\$ ', \mathrm{I} 4 / 40 \mathrm{X}, 55\left({ }^{\prime}-'\right) / / 36 \mathrm{X}$, 'CONSTANT HEAD NODES',57X, 'LEAKAGE' / CHK 650

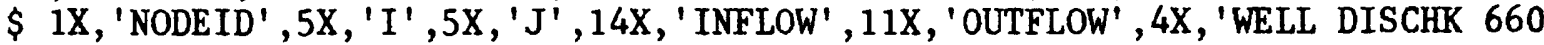
\$CHARGE' , 10X, 'RECHARGE' , 6X, 'STEADY-STATE' , 9X, 'TRANSIENT' )

320 CONTINUE

$\mathrm{ND}=0$

DO $230 \quad I=2$, DIML

DO $230 \mathrm{~J}=2$, DIMW

IF $(\mathrm{T}(\mathrm{I}, \mathrm{J}) . \mathrm{EQ} .0$.$) GO TO 230$

DO $330 \mathrm{~K}=1,6$

CHK 670

CHK 680

CHK 690

CHK 700

CHK 710

CHK 720

CHK 730

$330 \operatorname{XPT}(K)=0.0$ 
Table 1.--Listing of computer program for Laramie County model--Continued

IF (NODE.EQ.CHK(14)) ND=IABS (NODEID $(I, J))$

CHK 750

$\operatorname{AREA}=\operatorname{DELX}(\mathrm{J}) * \operatorname{DELY}(\mathrm{I})$

IF $(\mathrm{S}(\mathrm{I}, \mathrm{J}) . \mathrm{GE} .0$.$) GO TO 120$

C

C

---COMPUTE FLOW RATES TO AND FROM CONSTANT HEAD BOUNDARIES---

IF (S $(I, J-1) . L T .0 \ldots$. OR.T $(I, J-1) . E Q .0$.$) GO TO 30$

$\mathrm{X}=(\operatorname{STRT}(\mathrm{I}, \mathrm{J})-\mathrm{PHI}(\mathrm{I}, \mathrm{J}-1)) * \mathrm{TR}(\mathrm{I}, \mathrm{J}-1) * \operatorname{DELY}(\mathrm{I})$

IF (X) $10,30,20$

$10 \mathrm{CHD} 1=\mathrm{CHD} 1+\mathrm{X}$

IF (ND.NE.0) XPT (2) $=X P T(2)+X$

GO TO 30

$20 \mathrm{CHD} 2=\mathrm{CHD} 2+\mathrm{X}$

IF (ND.NE.0) XPT (1) $=X P T(1)+X$

30 IF $(\mathrm{S}(\mathrm{I}, \mathrm{J}+1)$.LT.0..OR.T $(\mathrm{I}, \mathrm{J}+1)$.EQ.0. $)$ GO TO 60

$\mathrm{X}=(\operatorname{STRT}(\mathrm{I}, \mathrm{J})-\operatorname{PHI}(\mathrm{I}, \mathrm{J}+1)) * \operatorname{TR}(\mathrm{I}, \mathrm{J}) * \operatorname{DELY}(\mathrm{I})$

IF (X) $40,60,50$

$40 \mathrm{CHD} 1=\mathrm{CHD} 1+\mathrm{X}$

IF (ND.NE.0) XPT (2) $=X P T(2)+X$

GO TO 60

$50 \mathrm{CHD} 2=\mathrm{CHD} 2+\mathrm{X}$

IF (ND.NE.0) XPT (1) $=\mathrm{XPT}(1)+\mathrm{X}$

60 IF (S(I-1,J).LT.0..OR.T (I-1,J).EQ.0.) GO TO 90

$X=(\operatorname{STRT}(I, J)-\operatorname{PHI}(I-1, J)) \div T C(I-1, J) * \operatorname{DELX}(J)$

IF (X) $70,90,80$

$70 \mathrm{CHD} 1=\mathrm{CHD} 1+\mathrm{X}$

IF (ND.NE.0) XPT (2) $=\mathrm{XPT}(2)+\mathrm{X}$

GO TO 90

$80 \mathrm{CHD} 2=\mathrm{CHD} 2+\mathrm{X}$

IF (ND.NE.0) XPT (1) $=X P T(1)+X$

90 IF $(S(I+1, J) . L T .0$. OR.T $(I+1, J) . E Q .0$.$) GO TO 340$

$\mathrm{X}=(\operatorname{STRT}(\mathrm{I}, \mathrm{J})-\operatorname{PHI}(\mathrm{I}+1, \mathrm{~J})) \div \mathrm{TC}(\mathrm{I}, \mathrm{J}) \div \operatorname{DELX}(\mathrm{J})$

IF (X) $100,340,110$

$100 \mathrm{CHD} 1=\mathrm{CHD} 1+\mathrm{X}$

IF (ND.NE.0) XPT (2) $=\mathrm{XPT}(2)+\mathrm{X}$

GO TO 340

$110 \mathrm{CHD} 2=\mathrm{CHD} 2+\mathrm{X}$

IF (ND.NE.0) XPT (1) $=\mathrm{XPT}(1)+\mathrm{X}$

C

GO TO 340

C ---RECHARGE AND WELLS---

120 QREFLX=QREFLX+QRE $(\mathrm{I}, \mathrm{J}) \div \mathrm{AREA}$

IF (ND.NE.0) XPT (3)=WELL $(I, J) \div A R E A$

IF (ND.NE .0) XPT (4)=QRE (I,J) $\approx A R E A$

IF (WELL $(I, J)) \quad 130,150,140$

130 PUMP=PUMP+WELL $(I, J) \neq A R E A$

GO TO 150

140 CFLUX $=$ CFLUX+WELL $(I, J) * A R E A$

150 IF (EVAP.NE.CHK(6)) GO TO 180

C ---COMPUTE ET RATE---

IF $(P H I(I, J)$.GE.GRND $(I, J)$-ETDIST) GO TO 160

ETQ $=0.0$

GO TO 170

$160 \mathrm{ETQ}=Q \mathrm{QT} / \mathrm{ETDIST} \div(\operatorname{PHI}(\mathrm{I}, \mathrm{J})+\mathrm{ETDIST}-\mathrm{GRND}(\mathrm{I}, \mathrm{J}))$

170 ETFLUX=ETFLUX-ETQ*AREA
CHK 760

CHK 770

CHK 780

CHK 790

CHK 800

CHK 810

CHK 820

CHK 830

CHK 840

CHK 850

CHK 860

CHK 870

CHK 880

CHK 890

CHK 900

CHK 910

CHK 920

CHK 930

CHK 940

CHK 950

CHK 960

CHK 970

CHK 980

CHK 990

CHK 1000

CHK 1010

CHK 1020

CHK 1030

CHK 1040

CHK 1050

CHK 1060

CHK 1070

CHK 1080

CHK 1090

CHK 1100

CHK 1110

CHK 1120

CHK 1130

CHK 1140

CHK 1150

CHK 1160

CHK1170

CHK 1180

CHK 1190

CHK 1200

CHK 1210

CHK 1220

CHK 1230

CHK 1240

CHK 1250

CHK 1260

CHK 1270

CHK 1280

CHK 1290 
Table 1.-- - Listing of computer program for Laramie County model--Continued

C

C ---COMPUTE VOLUME FROM STORAGE---

180 STORE $=S(I, J)$

IF (WATER.EQ.CHK(2)) STORE=SY $(I, J)$

IF (CONVRT.NE.CHK(7)) GO TO 220

$\mathrm{X}=\operatorname{KEEP}(\mathrm{I}, \mathrm{J})-\mathrm{PHI}(\mathrm{I}, \mathrm{J})$

IF (X) $190,200,200$

$190 \mathrm{HED} 1=\mathrm{PHI}(\mathrm{I}, \mathrm{J})$

HED2 $=\operatorname{KEEP}(I, J)$

$\mathrm{X}=\mathrm{ABS}(\mathrm{X})$

GO TO 210

200 HED $1=\operatorname{KEEP}(\mathrm{I}, \mathrm{J})$

HED2 $=\operatorname{PHI}(I, J)$

210 STORE $=S(I, J)$

IF (HED1-TOP $(I, J) . L E .0$.$) STORE =S Y(I, J)$

IF $(($ HED1-TOP $(I, J)) *($ HED2-TOP $(I, J)) \cdot \operatorname{LT} .0 .0)$ STORE $=(H E D 1-T O P(I, J)) / C H$ $1 X * S(I, J)+(T O P(I, J)-H E D 2) / X * S Y(I, J)$

$\mathrm{C}$

$220 \mathrm{STOR}=\mathrm{STOR}+\operatorname{STORE} *(\operatorname{KEEP}(I, J)-\operatorname{PHI}(I, J)) * A R E A$

C ---COMPUTE LEAKAGE RATE---

IF (LEAK.NE.CHK(9)) GO TO 340

IF $(M(I, J) . E Q .0$.$) GO TO 340$

HED $1=\operatorname{STRT}(I, J)$

IF (CONVRT.EQ.CHK(7)) HED $1=\operatorname{AMAX} 1(\operatorname{STRT}(I, J), \operatorname{TOP}(I, J))$

HED2 $=$ PHI $(I, J)$

IF (CONVRT.EQ.CHK( 7)) HED2=AMAX1(SNGL $(\operatorname{PHI}(I, J)), \operatorname{TOP}(I, J))$

$X X=\operatorname{RATE}(I, J) *(\operatorname{RIVER}(I, J)-\operatorname{HED} 1) * \operatorname{AREA} / \mathrm{M}(\mathrm{I}, \mathrm{J})$

IF (TL $(I, J) . E Q .0 .0) \quad X X=0.0$

$\mathrm{YY}=\mathrm{TL}(\mathrm{I}, \mathrm{J}) *($ HED $1-\mathrm{HED} 2) * \mathrm{AREA}$

FLUX $=F L U X+X X$

$\mathrm{XNET}=\mathrm{XX}+\mathrm{YY}$

IF (ND.NE.0) XPT(5) $=X X$

IF (ND.NE.0) XPT (6) $=Y Y$

FLUXS $=$ FLUXS + XNET

IF (XNET . LT . O.) FLXN=FLXN-XNET

$340 \mathrm{KTST}=0$

IF (KPH.EQ.0) GO TO 341

IF (MOD (KP, KPH).NE.0) GO TO 230

341 DO $350 \mathrm{~K}=1,6$

350 IF (ND.NE.0.AND. XPT (K).NE.0.0) KTST $=K T S T+1$

IF (KTST.EQ.0) GO TO 230

IF ((MOD (KT,KTH) .EQ. 0.OR.IFINAL.NE .0).AND. IO.NE.I) WRITE $(P, 3610)$

3610 FORMAT (1HO)

$\mathrm{IO}=\mathrm{I}$

IF (MOD(KT,KTH) .EQ. 0.OR.IFINAL.NE.0) WRITE (P, 3620) ND, I,J,

$\$(X P T(K), K=1,6)$

3620 FORMAT $(3 \mathrm{X}, 3(\mathrm{I} 4,2 \mathrm{X}), 1 \mathrm{P} 6 \mathrm{E} 18.3)$

DO $420 \mathrm{JN}=1$, NMBR

IF (ND.NE.NOD(JN)) GO TO 420

DO $470 \quad$ IN $=1,6$

470 QFLX (IN, JN) =QFLX (IN, JN) +XPT (IN)

420 CONTINUE

230 CONTINUE

IF (NODE.NE.CHK(14)) GO TO 441

IF (KPH.EQ.0) GO TO 429
CHK 1300

CHK1310

CHK1320

CHK1330

CHK 1340

CHK 1350

CHK1360

CHK1370

CHK1380

CHK 1390

CHK 1400

CHK 1410

CHK1420

CHK 1430

CHK 1440

CHK 1450

CHK1460

CHK 1470

CHK1480

CHK 1490

CHK 1500

CHK15 10

CHK1520

CHK 1530

CHK 1540

CHK1550

CHK 1560

CHK 1570

CHK1580

CHK 1590

CHK 1600

CHK 1610

CHK 1620

CHK 1630

CHK 1640

CHK 1650

CHK 1660

CHK 1670

CHK 1680

CHK1690

CHK 1700

CHK1 710

CHK1720

CHK1730

CHK1740

CHK 1750

CHK 1760

CHK 1770

CHK1780

CHK1790

CHK1 800

CHK1810

CHK 1820

CHK1830

CHK 1840 
Table 1.--Listing of computer program for Laramie County model--Continued

IF (MOD (KP,KPH).NE.0) GO TO 441

429 IF (MOD (KT,KTH).NE.0.AND.IFINAL.EQ.0) GO TO 441

WRITE $(P, 430)$

430 FORMAT $(4(/), 13 \mathrm{X}$, 'NODEID' $4 \mathrm{X}$, 'SUM OF FLOW RATES' //)

DO $440 \mathrm{JN}=1$, NMBR

$440 \operatorname{WRITE}(\mathrm{P}, 450)$ NOD (JN), (QFLX (IN, JN), IN=1,6)

450 FORMAT (15X, I4, 2X, 1P6E 18.3$)$

C

441 IF (STDY.EQ.CHK(15).AND.KT.EQ.0) RETURN

C ---COMPUTE CUMULATIVE VOLUMES, TOTALS, AND DIFFERENCES---

STORT $=$ STORT + STOR

STOR=STOR/DELT

ETFLXT=ETFLXT-ETFLUX*DELT

FLUXT $=$ FLUXT + FLUXS $*$ DELT

FLXNT $=$ FLXNT $+F L X N * D E L T$

FLXPT $=$ FLUXT + FLXNT

QRET $=Q R E T+Q R E F L X \div D E L T$

CHK 1850

CHK 1860

CHK 1870

CHK 1880

CHK 1890

CHK 1900

CHK 1910

CHK1920

CHK 1930

CHK 1940

CHK1950

CHK 1960

CHK 1970

CHK1980

CHK 1990

CHK2000

$\mathrm{CHDT}=\mathrm{CHDT}-\mathrm{CHD} 1 * \mathrm{DELT}$

CHK2010

CHST $=$ CHST + CHD $2 *$ DELT

CHK2020

PUMPT $=$ PUMPT - PUMP $* D E L T$

CHK2030

CFLUXT $=$ CFLUXT + CFLUX $*$ DELT

CHK2040

TOTL $1=$ STORT + QRET + CFLUXT + CHST + FLXPT

CHK2050

TOTL2 $=$ CHDT + PUMPT + ETFLXT + FLXNT

SUMR $=$ QREFLX + CFLUX +CHD2 +CHD 1 + PUMP +ETFLUX +FLUXS +STOR

CHK2060

DIFF $=$ TOTL2 - TOTL 1

CHK2070

PERCNT $=0.0$

IF (TOTL2.EQ.0.) GO TO 240

PERCNT $=$ DIFF $/$ TOTL $2 * 100$.

CHK2080

CHK2090

CHK2 100

CHK 2110

240 RETURN

CHK2120

CHK2130

C $\ldots \ldots \ldots$

CHK 2140

---PRINT RESULTS---

CHK2 150

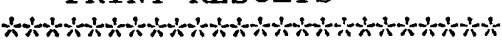

CHK2 160

ENTRY CWRITE

CHK2 170

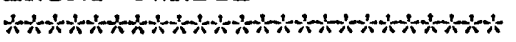

CHK2180

CHK2 190

CHK2200

WRITE (P, 250) STOR, QREFLX, STORT , CFLUX, QRET, PUMP , CFLUXT, ETFLUX, CHSTCHK2 210 1 , FLXPT , CHD2, TOTL1, CHD 1 , FLUX , FLUXS , ETFLXT , CHDT , SUMR , PUMPT , FLXNT , TOTCHK2220 2L2, DIFF, PERCNT

CHK2230

RETURN

CHK2240

CHK2250

---FORMATS---

CHK2260

CHK2270

CHK2280

CHK2290

250 FORMAT ('0', 10X, 'CUMULATIVE MASS BALANCE:', 16X, 'L $* * 3$ ', 23X, 'RATES FCHK2300 10R THIS TIME STEP: ', 16X, 'L*'*3/T'/11X,24('-') ,43X,25('-')//20X, 'SOUCHK2310

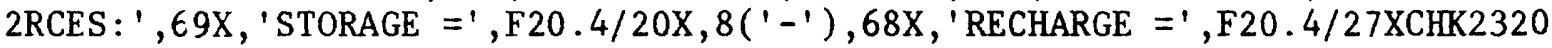
3, ' STORAGE $=$ ',F20.2,35X, 'CONSTANT FLUX $=$ ' ,F20.4/26X, 'RECHARGE $=$ ', F2CHK2330 40.2,41X, 'PUMPING =' ,F20.4/21X, 'CONSTANT FLUX =' ,F20.2, 30X, ' EVAPOTRCHK2340 5ANSPIRATION $=', \mathrm{~F} 20.4 / 21 \mathrm{X},{ }^{\prime}$ CONSTANT HEAD $=', \mathrm{~F} 20.2,34 \mathrm{X},{ }^{\prime}$ CONSTANT HEACHK2350 6D: ' $/ 27 \mathrm{X}$, 'LEAKAGE $=$ ' ,F20.2, 46X, ' IN =' ,F20.4/21X, ' TOTAL SOURCES =', FCHK2360 $720.2,45 \mathrm{X}$, 'OUT $=$ ' ,F20.4/96X, 'LEAKAGE: '//20X, 'DISCHARGES : ' , 45X, ' FROM CHK2370

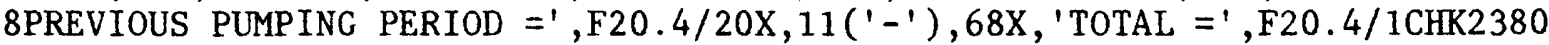
96X, 'EVAPOTRANSPIRATION $=$ ', F20.2/21X, 'CONSTANT HEAD $=$ ' ,F20.2, 36X, ' SCHK2390 
Table 1.--Listing of computer program for Laramie County model--Continued

\$UM OF RATES $=$ ', F20.4/19X' QUANTITY PUMPED =',F20.2/27X, 'LEAKAGE =' ,CHK2400 $\$ F 20.2 / 19 X,{ }^{\prime}$ TOTAL DISCHARGE $=', F 20.2 / / 17 X, '$ DISCHARGE-SOURCES $='$, F20CHK2410 $\$ .2 / 15 \mathrm{X},{ }^{\prime}$ PER CENT DIFFERENCE $=', F 20.2 / /$ )

CHK2420

END

CHK2430

SUBROUTINE PRNTAI(PHI, STRT, T, S, WELL, DELX, DELY) PRT 10



$\begin{array}{lr}\text { PRINT MAPS OF DRAWDOWN AND HYDRAULIC HEAD } & \text { PRT } 30\end{array}$

$\mathrm{C}$

PRINT MAPS OF DRAWDOWN AND HYDRAULIC HEAD

PRT 40

C

C SPECIFICATIONS:

PRT 50

REAL *8PHI , Z, XLABEL, YLABEL, TITLE , XN1, MESUR

PRT 60

REAL *4K

PRT 70

PRT 80

C

DIMENSION PHI (IO, JO,KO), STRT (IO, JO,KO), S(IO, JO, KO), WELL(IO, JO,KPRT 100

C

10), $\operatorname{DELX}(\mathrm{JO}), \operatorname{DELY}(\mathrm{IO}), \mathrm{T}(\mathrm{IO}, \mathrm{J} 0, \mathrm{KO})$

PRT 110

PRT 120

COMMON / INTEGR/ I0 , J0 , K0 , I 1 , J1 , K1 , I , J , K, NPER, KTH , ITMAX, LENGTH , KP , NPRT 130 1WEL , NUMT , IF INAL , IT , KT , IHEAD, IDRAW, IFLO, IERR , I2 , J2 , K2 , IMAX , ITMX1 , NCPRT 140 2H, IDK1 , IDK2 , IWATER, IQRE, IP , JP , IQ, JQ, IK , JK, K5, IPU1, IPU2, ITK, IEQN PRT 150 COMMON /PR/ XIAABEL (3), YLABEL (6), TITLE (6), XN1, MESUR, PRNT (122), BLANKPRT 160 1(60), DIGIT (122), VF 1(6), VF2 (6), VF3 (7), XSCAIE , DINCH, SYM (17), XN(100), PRT 170 $2 \mathrm{YN}(13), \mathrm{NA}(4), \mathrm{N} 1, \mathrm{~N} 2, \mathrm{~N} 3$, YSCALE , FACT1 , FACT2 RETURN

PRT 180

PRT 190

$\mathrm{C}$ ........

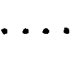

---INITIALIZE VARIABLES FOR PLOT---

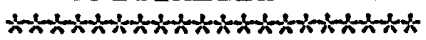

ENTRY MAP

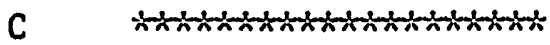

YDIM $=0$.

WIDTH $=0$.

DO $10 \mathrm{~J}=2, \mathrm{~J} 1$

10 WIDTH=WIDTH+DELX $(\mathrm{J})$

DO $20 \mathrm{I}=2, \mathrm{I} 1$

20 YDIM=YDIM+DELY (I)

$30 \mathrm{XSF}=\mathrm{DINCH} \div \mathrm{XSCALE}$

YSF $=$ DINCH* $*$ SCALE

NYD=YDIM/YSF

IF (NYD*YSF . IE. YDIM-DELY (I1)/2.) NYD $=\mathrm{NYD+1}$

PRT 200

PRT 210

PRT 220

PRT 230

PRT 240

PRT 250

PRT 260

PRT 270

PRT 280

PRT 290

PRT 300

PRT 310

PRT 320

PRT 330

PRT 340

IF (NYD.LE. 12) GO TO 40

PRT 350

$\mathrm{DINCH}=\mathrm{YDIM} /(12 . \approx \mathrm{YSCALE})$

PRT 360

WRITE $(6,330)$ DINCH

IF (YSCALE.LT.1.0) WRITE $(6,340)$

GO TO 30

$40 \mathrm{NXD}=$ WIDTH $/ \mathrm{XSF}$

IF (NXD*XSF. LE. WIDTH-DELX(J1)/2.) $\mathrm{NXD}=\mathrm{NXD}+1$

PRT 370

PRT 380

PRT 390

PRT 400

PRT 410

PRT 420

$\mathrm{N} 4=\mathrm{NXD} * \mathrm{~N} 1+1$

$\mathrm{N} 5=\mathrm{NXD}+1$

$\mathrm{N} 6=\mathrm{NYD}+1$

$\mathrm{N} 8=\mathrm{N} 2 * \mathrm{NYD}+1$

$N A(1)=N 4 / 2-1$

$N A(2)=N 4 / 2$

$\mathrm{NA}(3)=\mathrm{N} 4 / 2+3$

PRT 430

PRT 440

PRT 450

PRT 460

PRT 470

PRT 480

$\mathrm{NC}=(\mathrm{N} 3-\mathrm{N} 8-10) / 2$

PRT 490

PRT 500 
Table 1.--Iisting of computer program for Laramie County model--Continued

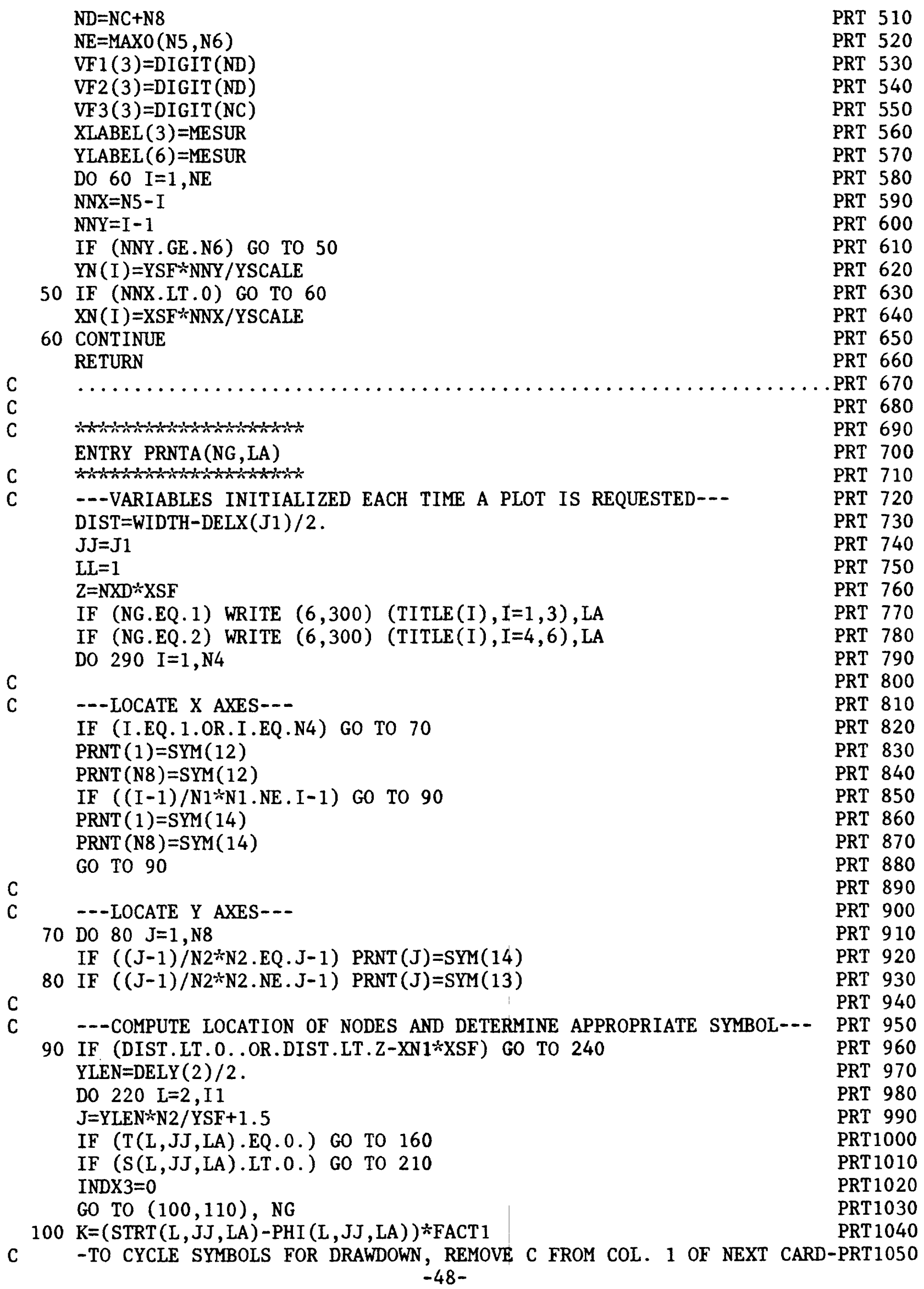


Table 1.--Listing of computer program for Laramie County model--Continued

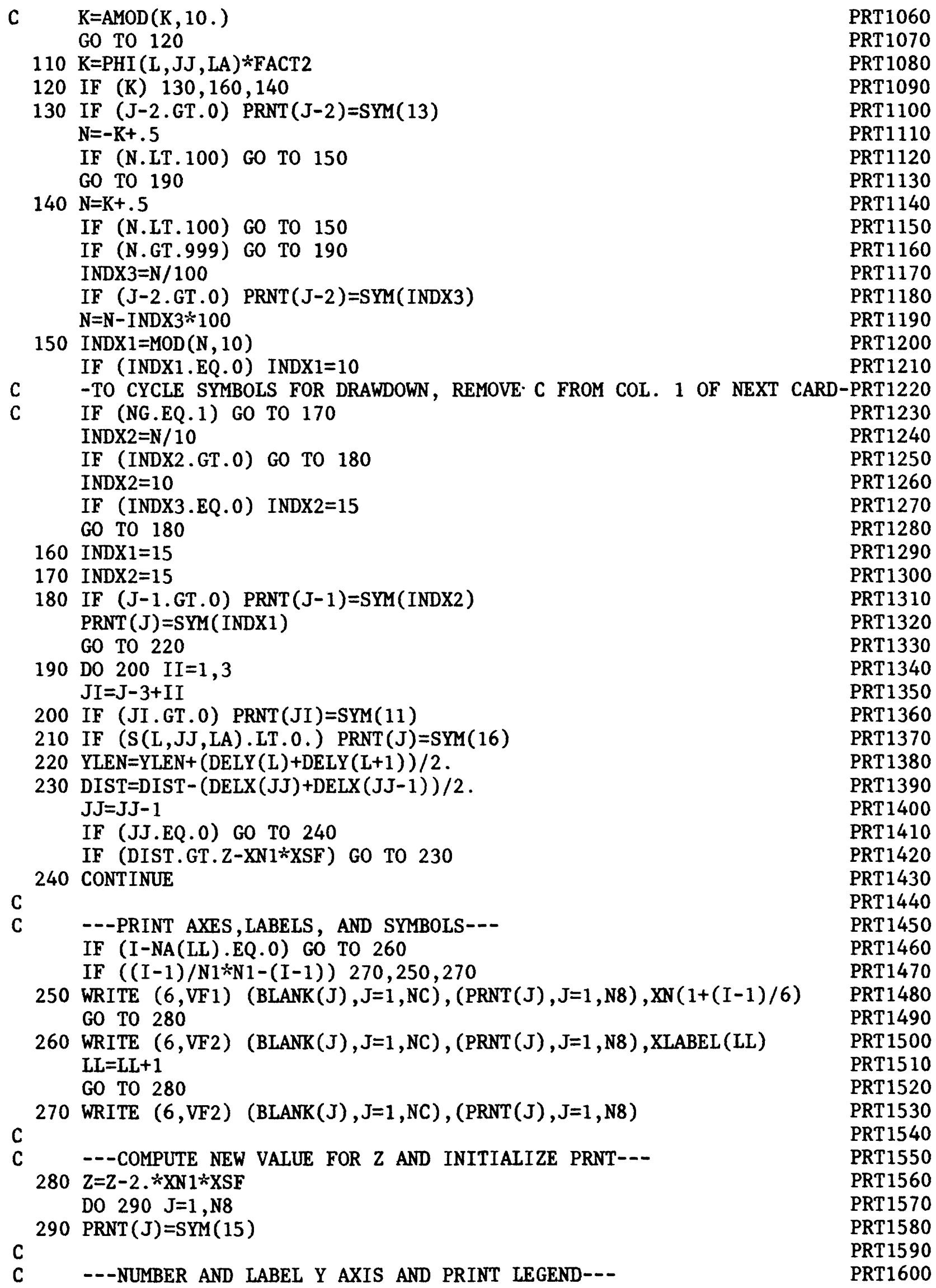


Table 1.--Listing of computer program for Laramie County model--Continued

WRITE $(6, \mathrm{VF} 3)(\operatorname{BLANK}(\mathrm{J}), \mathrm{J}=1, \mathrm{NC}),(\mathrm{YN}(\mathrm{I}), \mathrm{I}=1, \mathrm{~N} 6)$

PRT 1610

WRITE $(6,320)$ (YLABEL $(I), I=1,6)$

IF (NG.EQ.1) WRITE $(6,310)$ FACT1

PRT 1620

IF (NG.EQ.2) WRITE $(6,310)$ FACT2

PRT 1630

RETURN

PRT 1640

PRT 1650

C

C

---FORMATS---

PRT 1660

PRT 1670

PRT 1680

C

$\mathrm{C}$

C

300 FORMAT (' 1 ' , 49X, 3A8, 'LAYER' , I 4//)

PRT 1690

PRT 1700

PRT1710

PRT1720

310 FORMAT ('OEXPLANATION' $/$ ' ', $11\left({ }^{\prime}-'\right) / /$ ' $\mathrm{R}=$ CONSTANT HEAD BOUNDARY' /PRT 1730 $1^{\prime}{ }^{\prime}{ }^{\prime}+{ }^{\prime}=$ VALUE EXCEEDED 3 FIGURES' $/ '$ MULTIPLICATION FACTOR =',F8.3)PRT1740

320 FORMAT (' 0 ' , 39X, 6A8)

330 FORMAT (' 0 ', $25 X, 10\left(^{\prime} * '\right)$,' TO FIT MAP WITHIN 12 INCHES, DINCH REVISPRT 1760 1ED TO', G15.7,1X,10(' '*'))

340 FORMAT $\left(' 0\right.$ ', 45X,' NOTE : GENERALLY SCALE SHOULD BE I OR $\left.=1.0^{\prime}\right)$ END

PRT 1770

PRT 1780

PRT 1790

\section{BLOCK DATA}

C

COMMON /DPARAM/ RHO, B,D,F,H

BLK 10

COMMON /SARRAY/ TEST3(102),

BLK 20

1 AK, RECH , SIP , U, SS , TT , TMIN , ETDIST, QET, ERR, TMAX, CDLT , HMAX, YDIM, WIDTH, BLK 60

2NUMS , LSOR, ADI , DELT , SUM , SUMP , SUBS , STORE , TE ST, ETQB , ETQD, FACTX, FACTY , BLK 70

3IERR, KOUNT , IFINAL , NUMT , KT , KP , NPER , KTH, ITMAX, LENGTH , NWEL , NW, DIML , DIBLK 80

4MW, JNO1, INO1 , R , P , PU, I , J , NODE , STDY, KPH, IQPNC

BLK 90

COMMON /PR/ XLABEL(3), YLABEL (6), TITLE (5), XN1, MESUR, PRNT( 122), BLANKBLK 100 $1(60), \operatorname{DIGIT}(122), \operatorname{VF} 1(6), \operatorname{VF} 2(6), \operatorname{VF} 3(7), \operatorname{XSCALE}, \operatorname{DINCH}, \operatorname{SYM}(17), \operatorname{XN}(100), \operatorname{BLK} 110$ $2 \mathrm{YN}(13), \mathrm{NA}(4), \mathrm{N} 1, \mathrm{~N} 2, \mathrm{~N} 3, \mathrm{YSCALE}, \mathrm{FACT} 1, \mathrm{FACT} 2$

BLK 120

COMMON /ARSIZE/ IZ, JZ, IP , JP, IR, JR, IC , JC, IL , JL, IS , JS , IH, IMAX, IU, JU BLK 130

COMMON /NDID/ NOD $(100)$, NMBR

BLK 140

C

REAL $* 8 X L A B E L$, YLABEL, TITLE, XN 1 , MESUR, RHO , B , D , F , H

BLK 150

INTEGER R, P , PU, DIML , DIMW, CHK, WATER , CONVRT , EVAP , CHCK , PNCH , NUM, HEAD , BLK 170

1CONTR, LEAK, RECH , SIP , ADI , STDY

BLK 180

$\mathrm{C}$

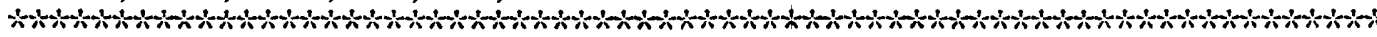

BLK 190

C

DATA IZ, JZ, IP , JP, IR , JR, IC, JC , IL , JL, IS , JS , IU, JU, IMAX/15*20/, IH/1/ BLK 210

DATA CHK/'PUNC', 'WATE', ' CONT', 'NUME', 'CHEC', 'EVAP' , 'CONV', 'HEAD' , 'BLK 220

1LEAK' , 'RECH' , 'SIP ', 'LSOR', 'ADI', 'NODE' , 'STDY'/, $\quad$ BLK 230

2R,P,PU/5,6,7/,B,D,F,H/4*0.DO/

BLK 240

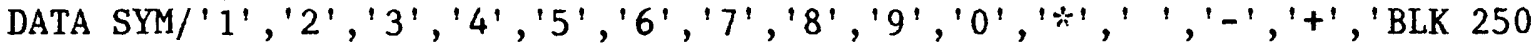

1 ', 'R', 'W' /

BLK 260

DATA PRNT/122*' $/ /, \mathrm{N} 1, \mathrm{~N} 2, \mathrm{~N} 3, \mathrm{XN} 1 / 6,10,133, .833333333 \mathrm{D}-1 /, \mathrm{BLANK} / 60 * '$ BLK 270

$1 \%$, NA(4)/1000/

BLK 280

DATA XLABEL/' X DIS- ', 'TANCE IN',' MILES '/,YLABEL/'DISTANCE',' BLK 290

1FROM OR', 'IGIN IN ', 'Y DIRECT', 'ION, IN ', 'MILES ' 2OF ', 'DRAWDOWN' , 'PLOT OF ', 'HYDRAULI', 'C HEAD' $/$

BLK 310

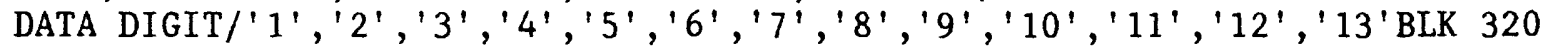

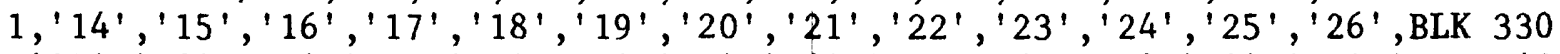

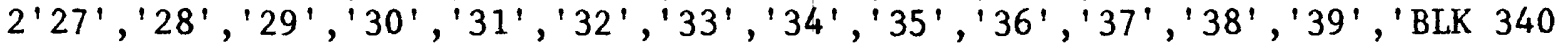

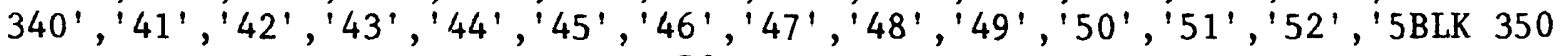
$-50-$ 
Table 1.--Listing of computer program for Laramie County model--Continued

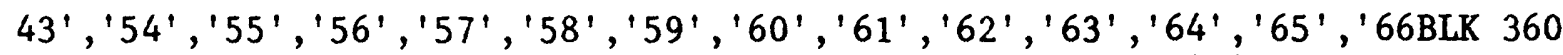

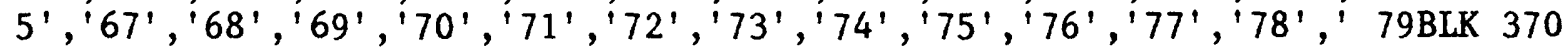

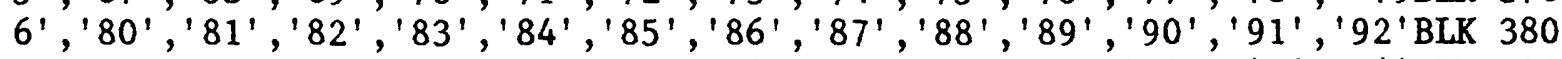
7, '93', '94', '95', '96', '97', '98', '99', '100',' '101', '102', '103', ' $104^{\prime}$ BLK 390

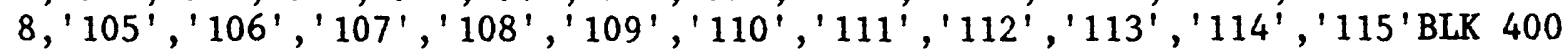
$9, ' 116^{\prime}, ' 117^{\prime},{ }^{\prime} 118^{\prime}, ' 119^{\prime}, ' 120^{\prime}, ' 121^{\prime}, ' 122^{\prime} /$

DATA VF1/'(1H ',',',' ', 'Al,F', '10.2',' ')'

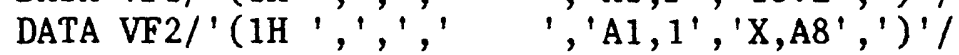

BLK 410

DATA VF3/'(1H0',',',' ','A1,F', $\left.3.1,{ }^{\prime}, ' 12 \mathrm{~F} 1^{\prime},{ }^{\prime} 0.2\right)^{\prime} /$

BLK 420

DATA VF 4/' (1H0',',I2,', '2X, ','16F8','.1/',' (5X,', '16F8','.1))', BLK 450 $\$ 3 \div+1 /$

DATA NOD $/ 100 * 0 /$

BLK 460

DATA PRINT/122*-

BLK 470

C

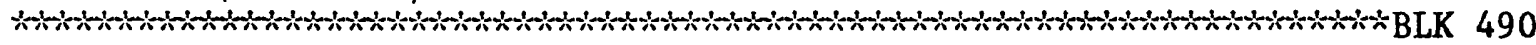

END

BLK 500 
Table 2.--Iisting of data for 1920-70

Card

number

Group I: Title, Simulation options, and problem dimensions

1.

2. TER SYSTEM $1920-1970$

LARAMIE COUNTY POST-CRETACEOUS GROUND-WA

3. WATE

4. IEAK

5. CONV

6. BLNK

7. RECH

8. SIP

9. CHEC

10. PUNC

11. NUME

12. HEAD

13. NODE

14. BLNK

15. $52 \quad 55$

Group II: Scalar parameters

16. BLNK

$17 . \quad 1$

18.

10

10

1.0

100

0.0

1

Group III: Array data

Grid spacing in $X$ direction, in feet

(First card is parameter card)

19.

20.

21.

22.

23.

24.

25 .

26 .

$\begin{array}{rr}1 & 1 \\ 10560 & 10560 \\ 5280 & 5280 \\ 5280 & 5280 \\ 5280 & 5280 \\ 5280 & 5280 \\ 5280 & 5280 \\ 5280 & 5280\end{array}$

0
10560
5280
5280
5280
5280
5280
7920

10560

5280

5280

5280

5280

5280

5280

5280

5280

5280

5280

5280

5280

5280

5280

5280

5280

5280

5280

5280

5280

5280

5280

5280

5280

5280

7920

16880

5280

5280

5280

Group III: Array data--Continued

Grid spacing in $Y$ direction, in feet

(First card is parameter card)

27 .

28.

29 .

30 .

31 .

32.

33.

1

7920

1
7920

5280

5280

5280

5280

5280

5280

5280

5280

5280

5280

5280

5280

5280

5280

5280

5280

5280

5280

5280

5280

5280

34. 
Table 2.--Listing of data for 1920-70--Continued

Card
number

35.

36.

37.

38.

39.

40.

41.

42.

43.

44.

45 .

46.

47.

48.

49.

50.

51.

52.

53.

54.

55.

56.

57.

58.

59.

60.

61.

62.

63.

64.

65.

66.

67.

68.

69.

70 .

71 .

72 .

73.

74.

75.

76 .

77.

78.

79.

80.

81 .

82.

83.
Group III: Array data--Continued

Node identification

(First card is parameter card)

10

10

$\begin{array}{lllll}10 & 10 & 10 & 10 & 10\end{array}$

$10 \quad 10$

10

10 $\begin{array}{lllllll}15 & 15 & 15 & 10 & 10 & 10 & 10\end{array}$

15

$15 \quad 15$

$\begin{array}{rrrr}10 & 10 & 10 & 10\end{array}$

15

$10 \quad 10 \quad 10$

0 15

$\begin{array}{llllllll}10 & 10 & 10 & 10 & 10 & 10 & 10 & 10\end{array}$

10

$\begin{array}{lll}10 & 10 & 10\end{array}$

$10 \quad 10$ 
Table 2.--Listing of data for 1920-70--Continued

Card

number

$\begin{array}{ll}84 . & 0 \\ 85 . & 0 \\ 86 . & 0 \\ 87 . & 0 \\ 88 . & 0 \\ 89 . & 0 \\ 90 . & 0 \\ 91 . & 0 \\ 92 . & 0 \\ 93 . & 0 \\ 94 . & 0 \\ 95 . & 0 \\ 96 . & 0 \\ 97 . & 0 \\ 98 . & 0 \\ 99 . & 0 \\ 100 . & 0 \\ 101 . & 0 \\ 102 . & 0 \\ 103 . & 0 \\ 104 . & 0 \\ 105 . & 0 \\ 106 . & 0 \\ 107 . & 0 \\ 108 . & 0 \\ 109 . & 0 \\ 110 . & 0 \\ 111 . & 0 \\ 112 . & 0 \\ 113 . & 0 \\ 114 . & 0 \\ 115 . & 0 \\ 116 . & 0 \\ 117 . & 0 \\ 118 . & 0 \\ 119 . & \\ 120 . & 0 \\ 121 . & 0 \\ 122 . & \\ 123 . & 0 \\ 124 . & 0 \\ 125 . & \end{array}$

125 .

126.

127.

128.

129.

130 .

131.

132.

133.
Group III: Array data--Continued

Node identification--Continued
0

0

0

0

0

0

0

0

0

0

0

0

0

0

0

0

0

0


Table 2.--Listing of data for 1920-70--Continued

Card

number

134.

135.

136.

137.

138.

139.

140.

141.

142.

143.

144.

145.

146.

147.

148.

149.

150.

151.

152.

153.

154.

155.

156.

157.

158.

159 .

160.

161.

162.

163.

164.

165.

166.

167.

168.

169.

170 .

171 .

172.

173.

174.

175 .

176.

177.

178 .

179 .

180 .

181.

182.

183.
Group III: Array data--Continued

Node identification--Continued

30 35

$\begin{array}{llllllllll}30 & 30 & 30 & 30 & 30 & 30 & 30 & 30 & 30 & 30\end{array}$

0

0

0

30

0

$\begin{array}{llllll}30 & 30 & 30 & 30 & 30 & 30\end{array}$

0

$\begin{array}{lll}0 & 30 \quad 30 \\ 0 & & \end{array}$

0

0

30

0

30

0

0

30

0

0

30

0

0

30

0

0

0

30

0

30

0

0

30

0

0

30

0

0

0

0

30 
Table 2.--Listing of data for 1920-70--Continued

Card

number

184.

185.

186.

187.

188.

189 .

190.

191.
Group III: Array data--Continued

Node identification--Continued

Explanation of node identification

192. 10 HORSE CREEK

193. 15 LITTLE HORSE CREEK

194. 20 LODGEPOLE CREEK

195. 30 CROW CREEK

196. 35 CHEYENNE MUNICIPAL DISCHARGE

197.0

Elapsed time, in seconds and cumulative volumes, in cubic feet for mass balance

198.

199.

200 . $\begin{array}{ll}0 & 0 \\ 0 & 0 \\ 0 & 0\end{array}$
0

0

\section{Group III: Array data--Continued \\ Starting head matrix, in feet \\ (First card is parameter card)}

\begin{tabular}{|c|c|c|c|c|c|c|c|c|}
\hline 201. & 1 & 1 & 2 & & & & & \\
\hline 202. & 0.0 & 0.0 & 0.0 & 0.0 & 0.0 & 0.0 & 0.0 & 0.0 \\
\hline 203. & 0.0 & 0.0 & 0.0 & 0.0 & 0.0 & 0.0 & 0.0 & 0.0 \\
\hline 204. & 0.0 & 0.0 & 0.0 & 0.0 & 0.0 & 0.0 & 0.0 & 0.0 \\
\hline 205 . & 0.0 & 0.0 & 0.0 & 0.0 & 0.0 & 0.0 & 0.0 & 0.0 \\
\hline 206. & 0.0 & 0.0 & 0.0 & 0.0 & 0.0 & 0.0 & 0.0 & 0.0 \\
\hline 207. & 0.0 & 0.0 & 0.0 & 0.0 & 0.0 & 0.0 & 0.0 & 0.0 \\
\hline 208 . & 0.0 & 0.0 & 0.0 & 0.0 & 0.0 & 0.0 & 0.0 & \\
\hline 209. & 0.0 & 0.0 & 0.0 & 0.0 & 0.0 & 0.0 & 0.0 & 0.0 \\
\hline 210. & 0.0 & 0.0 & 0.0 & 0.0 & 0.0 & 0.0 & 0.0 & 0.0 \\
\hline 211. & 5288.6540 & 5250.0000 & 5225.0000 & 5200.0000 & 5170.0000 & 5140.0000 & 5130.0000 & 5115.0000 \\
\hline 212 . & 5070.0000 & 5040.0000 & 5035.0000 & 5030.0000 & 5020.0000 & 5000.0000 & 5000.0000 & 5010.0000 \\
\hline 213. & 5010.0000 & 5000.0000 & 5025.6319 & 4975.0000 & 0.0 & 0.0 & 0.0 & 0.0 \\
\hline 214 . & 0.0 & 0.0 & 0.0 & 0.0 & 0.0 & 0.0 & 0.0 & 0.0 \\
\hline 215 . & 0.0 & 0.0 & 0.0 & 0.0 & 0.0 & 0.0 & 0.0 & \\
\hline 216 & 0.0 & 0.0 & 0.0 & 0.0 & 0.0 & 0.0 & 0.0 & 0.0 \\
\hline 21 & 0.0 & 0.0 & 0.0 & 0.0 & 0.0 & 0.0 & 0.0 & 0.0 \\
\hline
\end{tabular}


Table 2.--Listing of data for 1920-70--Continued

Card

number

218.

219 .

220 .

221 .

222.

223.

224.

225 .

226.

227 .

228 .

229 .

230 .

231 .

232.

233.

234.

235 .

236.

237.

238.

239 .

240 .

241.

242.

243.

244.

245 .

246 .

247.

248 .

249 .

250 .

251 .

252.

253.

254.

255.

256.

257.

258 .

259 .

260.

261.

262.

263.

264.

265.

266.

267.
Group III: Array data--Continued

Starting head matrix, in feet--Continued
5349.51475316 .9350

5152.85335133 .9709

5022.90165010 .9927

0.0

0.0

0.0

0.0

0.0

0.0

5424.50755399 .0829

5205.69055178 .4214

$5016.5245 \quad 4997.9448$

4788.25604764 .9373

4910.0000

$$
0.0
$$

0.0

5821.59845696 .3263

5464.24525437 .6359

$5223.0668 \quad 5192.8854$

$4970.0022 \quad 4954.8945$

4761.3495

4729.9960

4923.3998

0.0

4920.0902

6690.0000

5828.5980

5729.2945

5483.67975456 .2673

5224.05375192 .9768

4967.7351

4740.6214

4943.9196

0.0

5834.4002

5503.7667

5224.4389

4965.1388

4756.1156

4963.8031

0.0

5864.1630

5503.9772

5225.1510

5000.7347

4787.6616

5015.8067

0.0

5880.2155

5505.1261

5281.8605

5049.1783

4898.7812

5045.7457

0.0

5908.4562

5499.9935 4941.3048

4719.9910

4942.9287

6690.000066 5749.5627

5468.9840

5190.0337

4940.0685

4747.6103

4960.6965

6690.0000

\subsection{8}

5228.5713

4978.5443

4805.1019

5003.4856

6700.0000

5469.5669

5031.0505

5037.4329

6640.0000

5852.3791
5273.868752

4993.0609

5249.37685231 .0355

5215.7944

4968.9274

\begin{abstract}
0.0
\end{abstract}
0.0

0.0

0.0

0.0

0.0

0.0

0.0

0.0

0.0

0.0

$5540.0000 \quad 5450.0000 \quad 5450.0000 \quad 5450.0000 \quad 5450.0000$

$\begin{array}{llllll}5356.6987 & 5312.2055 & 5279.9076 & 5267.8652 & 5254.8059 & 5231.8783\end{array}$

$5150.19375118 .72695090 .6221 \quad 5073.3176 \quad 5058.3346 \quad 5035.0641$ $4961.5284 \quad 4914.6065 \quad 4890.0000 \quad 4860.0000 \quad 4836.8024 \quad 4809.0417$ $4713.6958 \quad 4670.0000 \quad 4680.0000 \quad 4740.0000 \quad 4850.0000 \quad 4900.0000$ $4850.0000 \quad 4780.0000 \quad 4750.00004730 .0000$

0.0

0.0

0.0

0.0

0.0

0.0

0.0

$\begin{array}{llllll}5626.6082 & 5582.5143 & 5540.8561 & 5526.8938 & 5519.2775 & 5495.9366\end{array}$ 5412.99935374 .01555330 .35405302 .83165280 .35645252 .4845 $\begin{array}{llllll}5159.5064 & 5124.0444 & 5093.3381 & 5069.9876 & 5035.0059 & 4995.0037\end{array}$ $\begin{array}{llllllll}4918.5499 & 4893.7494 & 4868.5819 & 4842.0868 & 4814.0918 & 4785.8827\end{array}$ $4694.68174685 .9557 \quad 4714.26624787 .3556$ $4898.56914849 .1208 \quad 4788.8874 \quad 4755.0000$ $\begin{array}{llll}6615.1210 & 6518.02096343 .6387 \quad 6231.8817\end{array}$

4872.95564

4914.3035

5664.56735617 .09065582 .6966556

5431.67425398 .63435359 .76985326 .2854 \begin{tabular}{l}
5163.66125130 .00715100 .01645079 .8077 \\
\hline
\end{tabular}

4914.8496

4887.9383

4860.64104831 .8735

4746.2799482

$4695.9498 \quad 4693.7985$

$4932.7018 \quad 4902.8220 \quad 4840.9798 \quad 4780.0000$
6611.1488

4824.6118

0.0

6092.55835953 .2590

5546.35045519 .9837

5294.21795258 .7324

$5031.3659 \quad 4991.6495$

4796.10534764 .5864

$4905.5175 \quad 4936.3338$

6611.14886521 .2438

6366.97946231 .3481 0.0

5690.7973

5640.1703

5607.30525582 .8501

$6106.5515 \quad 5966.5980$

5561.66755538 .2788

5438.1342

5404.3116

5368.67335333 .0314

$5296.7040 \quad 5260.3266$

4914.8221

5173.7795

5140.50465091 .0435

$5037.7861 \quad 4996.2884$

4746.3112

4752.1412

4855.06974825 .0776

$4795.1935 \quad 4772.0877$

4953.1912

4932.3212

4806.1281

4896.7898

6605.7713

6515.7118

6384.13736240 .8671

0.0

5720.4007

5665.5755

5629.75705603 .6027

6107.57025987 .8633

5436.8478
5219.8072

5400.0815

5365.47515328 .2566

$5576.2570 \quad 5542.2542$

4957.1027

5219.5172
4940.1520

5173.03385111 .7050

22195269.3754

4824.4318

4850.2525

4913.9752

4856.4312

4985.1330

4960.5870

4909.76014870 .0000

5753.9695

6496.2538

6376.3443

6249.1810

\section{5}

5267.0222

$\begin{array}{llll}5389.9961 & 5359.9887 & 5320.0597\end{array}$

575

508

5016.5365

5012.3477

5017.9334500
4943.7777496

5006.7491

414

4956.5994

6568.9941

4983.0590

634

4932.62624900 .0000

5049

5787.7022

5720.90185663 .04765622 .8635

6105.00695995 .7799

0.0

5583.10955541 .8344

$5357.2948 \quad 5346.1069$ 
Table 2.--Listing of data for 1920-70--Continued

Card number

268.

269.

270.

271.

272.

273.

274.

275 .

276.

277.

278 .

279 .

280 .

281 .

282.

283.

284.

285 .

286.

287 .

288 .

289.

290 .

291.

292.

293.

294.

295.

296.

297.

298.

299.

300 .

301.

302.

303.

304.

305.

306.

307.

308.

309.

310.

311.

312.

313.

314.

315 .

316.

317.
Group III: Array data--Continued Starting head matrix, in feet--Continued $\begin{array}{lllllllll}5338.9599 & 5326.4579 & 5304.1741 & 5267.8731 & 5200.1107 & 5192.9514 & 5163.5336 & 5136.1888\end{array}$ $\begin{array}{lllllllll}5108.9718 & 5095.5537 & 5078.8513 & 5086.9591 & 5069.2282 & 5087.2158 & 5089.6079 & 5070.4997\end{array}$

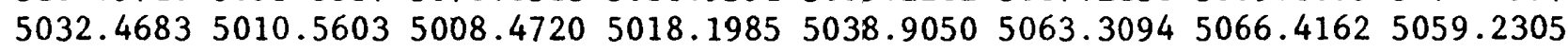
$\begin{array}{llllllll}5050.7817 & 5040.8630 & 5017.7930 & 4986.3916 & 4937.6404 & 4900.0000 & 0.0\end{array}$

$\begin{array}{llllllll}0.0 & 6590.0000 & 6528.1897 & 6403.8293 & 6317.3203 & 6209.0215 & 6106.0738 & 6005.6302\end{array}$ $\begin{array}{llllllll}5924.9488 & 5864.9899 & 5799.9379 & 5734.8890 & 5660.0057 & 5614.9908 & 5584.9646 & 5549.9725\end{array}$ $\begin{array}{lllllllll}5540.6927 & 5523.5491 & 5503.6397 & 5476.7224 & 5451.0059 & 5431.6500 & 5414.2031 & 5400.5537\end{array}$ $\begin{array}{llllllll}5391.6628 & 5377.7912 & 5351.0896 & 5309.8759 & 5286.5746 & 5268.5475 & 5244.5320 & 5218.5888\end{array}$

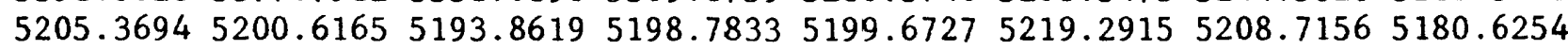
5141.25335114 .00535099 .56455093 .09635092 .45835090 .43785081 .33115069 .2548

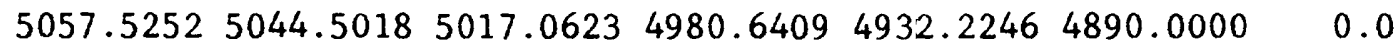

$\begin{array}{llllllll}0.0 & 6530.0000 & 6488.7494 & 6374.2463 & 6279.3614 & 6197.0046 & 6117.7119 & 6030.0149\end{array}$ $\begin{array}{lllllllll}5974.8833 & 5920.8186 & 5851.2907 & 5782.1367 & 5727.4580 & 5683.7032 & 5650.2723 & 5617.2694\end{array}$ $\begin{array}{lllllllll}5586.1230 & 5568.2222 & 5550.9110 & 5522.2048 & 5494.2848 & 5475.3822 & 5458.6150 & 5442.5664\end{array}$ $\begin{array}{llllllllll}5428.8864 & 5413.6799 & 5394.5649 & 5366.0307 & 5339.7939 & 5318.0628 & 5299.7918 & 5283.3669\end{array}$ $\begin{array}{llllllll}5281.6041 & 5277.0092 & 5274.9477 & 5270.1614 & 5265.8239 & 5254.6566 & 5237.5957 & 5214.1092\end{array}$ $\begin{array}{llllllll}5189.8517 & 5169.6152 & 5149.9430 & 5133.2791 & 5118.7390 & 5106.5307 & 5093.9617 & 5080.8676\end{array}$ $\begin{array}{lllllll}5066.0446 & 5052.6628 & 5015.3442 & 4966.5702 & 4924.0327 & 4870.0000 & 0.0\end{array}$

$\begin{array}{lllllllll}0.0 & 6470.0000 & 6442.1193 & 6353.1072 & 6259.9926 & 6190.0011 & 6125.0902 & 6097.2939\end{array}$ $\begin{array}{lllllllll}6043.2898 & 5980.0925 & 5898.9316 & 5837.8660 & 5787.5350 & 5745.7776 & 5709.2329 & 5678.8600\end{array}$ $\begin{array}{lllllllll}5642.4442 & 5615.1263 & 5591.8065 & 5563.4506 & 5531.8882 & 5510.3494 & 5490.0705 & 5471.5110\end{array}$ $\begin{array}{lllllllll}5452.0935 & 5434.4086 & 5414.4581 & 5391.9379 & 5370.0932 & 5350.7616 & 5334.8415 & 5319.7903\end{array}$ $\begin{array}{lllllllll}5307.9183 & 5298.5540 & 5290.8582 & 5282.3722 & 5272.6341 & 5259.4503 & 5242.5119 & 5221.3051\end{array}$ $\begin{array}{llllllll}5198.4996 & 5176.9376 & 5156.1825 & 5137.7252 & 5122.1783 & 5109.9473 & 5098.0142 & 5087.4963\end{array}$ $\begin{array}{llllllll}5073.1342 & 5050.9957 & 5008.1243 & 4957.0844 & 4918.3972 & 4860.0000 & 0.0\end{array}$

$\begin{array}{lllllllll}0.0 & 0.0 & 6400.0104 & 6345.0170 & 6299.0688 & 6245.6307 & 6189.1797 & 6162.7769\end{array}$ $6114.5208 \quad 6054.4718 \quad 5971.04295885 .1096 \quad 5842.0806 \quad 5804.46445766 .91345727 .5848$ $\begin{array}{lllllllll}5688.4544 & 5657.1599 & 5626.1761 & 5595.7559 & 5564.4393 & 5537.2839 & 5513.6595 & 5492.1882\end{array}$ $\begin{array}{llllllll}5471.6395 & 5451.8618 & 5431.6308 & 5410.7355 & 5390.3226 & 5371.4747 & 5354.5246 & 5339.3150\end{array}$ $\begin{array}{llllllllll}5326.0371 & 5314.6833 & 5303.5145 & 5292.2235 & 5279.6186 & 5265.0248 & 5248.2729 & 5228.6901\end{array}$ $\begin{array}{llllllll}5206.9028 & 5183.6697 & 5160.7478 & 5139.9352 & 5121.7589 & 5110.8513 & 5097.3414 & 5085.1952\end{array}$ $\begin{array}{lllllll}5071.8585 & 5046.5612 & 5002.0652 & 4951.3304 & 4912.8150 & 4855.0000 & 0.0\end{array}$

$\begin{array}{lllllllll}0.0 & 0.0 & 6430.0000 & 6410.2005 & 6341.0771 & 6302.6229 & 6251.2205 & 6216.1559\end{array}$ $\begin{array}{lllllllll}6172.5658 & 6116.7068 & 6037.1729 & 5942.3528 & 5876.3771 & 5844.7154 & 5808.9030 & 5769.6503\end{array}$ $\begin{array}{lllllllll}5726.7702 & 5687.7228 & 5651.3012 & 5618.6468 & 5588.1750 & 5558.1261 & 5533.1669 & 5510.9092\end{array}$ $\begin{array}{llllllllll}5489.5833 & 5468.7927 & 5448.1781 & 5427.7750 & 5407.8891 & 5388.9449 & 5371.0287 & 5354.0313\end{array}$ $\begin{array}{lllllllll}5338.5796 & 5324.9594 & 5312.1218 & 5298.7780 & 5284.8136 & 5269.5747 & 5253.2209 & 5234.0805\end{array}$ $\begin{array}{lllllllll}5213.0989 & 5191.4776 & 5168.5621 & 5146.1713 & 5123.7610 & 5104.7566 & 5089.1211 & 5079.3336\end{array}$ $\begin{array}{llllllll}5060.4798 & 5033.3206 & 4996.0962 & 4951.2120 & 4912.2194 & 4850.0000 & 0.0\end{array}$

$\begin{array}{lllllllll}0.0 & 6530.0000 & 6498.1077 & 6479.5884 & 6403.8388 & 6364.1933 & 6317.7641 & 6267.9056\end{array}$ $\begin{array}{llllllll}6217.1713 & 6156.9136 & 6067.5778 & 5975.0210 & 5909.0921 & 5864.7224 & 5828.9372 & 5791.0125\end{array}$ $\begin{array}{lllllllll}5749.8833 & 5709.3161 & 5673.0726 & 5637.9151 & 5605.2821 & 5576.2222 & 5550.1599 & 5527.4948\end{array}$ $\begin{array}{lllllllll}5505.6441 & 5484.3845 & 5463.5364 & 5443.1772 & 5423.3871 & 5404.2903 & 5385.4119 & 5366.0225\end{array}$ $\begin{array}{llllllll}5348.9352 & 5333.9801 & 5317.8615 & 5302.1411 & 5287.9431 & 5272.3612 & 5256.7364 & 5237.6342\end{array}$ $\begin{array}{lllllllll}5215.8696 & 5195.8760 & 5176.1294 & 5153.3358 & 5127.7197 & 5103.4061 & 5085.1864 & 5068.2771\end{array}$ $\begin{array}{rlllllll}5045.7550 & 5024.7890 & 4996.6926 & 4961.4562 & 4915.7931 & 4870.0000 & 0.0\end{array}$

$\begin{array}{lllllllll}0.0 & 6570.0000 & 6553.2384 & 6525.3716 & 6454.5784 & 6412.3859 & 6362.9228 & 6298.6635\end{array}$ $\begin{array}{llllllll}6246.1021 & 6180.6565 & 6093.9756 & 5994.3759 & 5921.0925 & 5880.6729 & 5840.7403 & 5801.5996\end{array}$ $\begin{array}{llllllllll}5766.9215 & 5730.3337 & 5692.8661 & 5655.8402 & 5621.6011 & 5592.8912 & 5566.6251 & 5542.4799\end{array}$

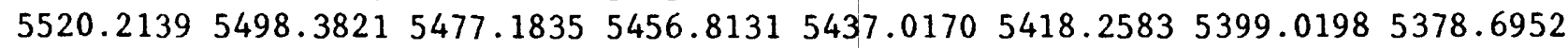


Table 2.--Listing of data for 1920-70--Continued

Card

number

318.

319 .

320 .

321 .

322.

323.

324.

325 .

326.

327.

328 .

329 .

330 .

331.

332.

333.

334.

335.

336.

337.

338 .

339 .

340 .

341 .

342.

343.

344.

345 .

346 .

347 .

348 .

349 .

350 .

351 .

352 .

353.

354 .

355 .

356.

357.

358 .

359 .

360 .

361 .

362.

363.

364 .

365 .

366.

367.
Group III: Array data--Continued Starting head matrix, in feet--Continued
$5358.24755341 .2672 \quad 5325.49285308 .15415291 .3303 \quad 5273.99285255 .83545235 .8444$ $5215.02005197 .61965182 .26895160 .2450 \quad 5133.9047 \quad 5108.2300 \quad 5083.66115062 .3378$ 5043.6123

0.0

5023.73584996 .7883

6595.00006630 .7148

6245.7993 6182.3976

5780.3379 5744.1521

5536.5012

5370.1435

5215.9512

5042.6243

0.0

5513.5164

6088.0101

4963.1793

4918.0396

4875.0000

6545.8555

6478.3200

6430.1308

0.0

6356.77376285 .1111

6220.5850

5350.3865

5491.5025

5995.7814

5934.7162

5884.8874

5469.87785449 .4009

5610.2411

5331.9790

5313.5000

5294.9802

5429.8792

5201.60405183 .7290
5023.8437

5160.368351

4964.1129

6625.00006649 .1311

6561.6094

59

4918.7179

5276.096

4870.0000

601

5790.7748

5158.6976

6092.2365

5015.4394

6484.0101

6411.1042

5556.2923

5531.2867

5721.8574

5483.5370

5656.9295

5890.5366

5460.9317

5627.5445

5378.4333

5219.0434

5043.5029

0.0

5203.3330

5338.3094

5318.7811

5298.8394

5441.3156

5849.81045815 .5204

5584.49535559 .8874

6206.4811

5025.4787

5184.1369

5160.2773

5135.1309

5278.6006

$5409.8090 \quad 5389.3464$

5256.42345235 .0614

6675.45996660 .0425

6565.0636

4918.5374

4865.0000

5569.7226

5757.7495

6093.8520

6018.8750

5944.9466

6407.7091

5082.

0.0

6334.64356275 .8307

5857.28095823 .5196

5603.25945579 .7579

5422.58735399 .7599

5258.22805237 .9353

5078.77575059 .6029 0.0

5389.3268

5219.4706

5545.9769

$5727.7530 \quad 5700.8902$

5672.2331

5898.1481

6329.54846261 .2496

5861.96705827 .1415

5045.0953

0.0

5367.7016

5347

19.10565495 .1437

5474.3084

5641.3906

5614.54915592 .2052

5200.70745180 .38465158 .37395134 .72525107 .1308

5027.972550

5003.0037

4968.1029

6194.7486

6141.1147

6083.0068

6575.1966

$6475.7336 \quad 6407.2815$

6009.2398

58

5574.7336

5757.7366

5731.882

5400.8762

5378.7747

5528.5906

5504.4570

1343

350

900.0907

5217.2644

5196.62555175 .8849

5050.7214

5030.2190

0.0

6723.8094667

5004.2081

5336.5019

5482.6639

5651.0802

6192.4042

5790.0583

5155.2130

5

684

6579.1258

5584.4738

5734.0512

5989.2325

35

950.5823

5708.7983

5683.2447

5492.4537

5514.2546

5320.7468

5212.0611

5065.0861

5387.93175366 .5996

5344.635

5134.3625

5043.05735011 .9523

5150.7183

$\begin{array}{ll}4967.5477 & 4917.5022 \\ 6577.9631 & 6485.9067\end{array}$

5941.6556

6069.60175998 .3387

5687.7215
5503.1137

5712.3685

5547.3347

5524.7387

5325.6547

5462.4118

5435.37045413 .1884

5260.11595239 .2876

5080.09605061 .1059 0.0

6332.02286256 .3386

$5861.7140 \quad 5827.1522$

5623.18845597 .3712

5444.64915424 .4801

5790.1916

5761.0626
5570.2517

5373.79605350 .6855

5297.7881

$\begin{array}{lllll}5161.0733 & 5143.9120 & 5127.4210 & 5112.5738 \\ 5018.1537 & 4967.5099 & 4916.4193 & 4865.0000\end{array}$

$\begin{array}{lllll}5161.0733 & 5143.9120 & 5127.4210 & 5112.5738 \\ 5018.1537 & 4967.5099 & 4916.4193 & 4865.0000\end{array}$

$\begin{array}{lllll}5161.0733 & 5143.9120 & 5127.4210 & 5112.5738 \\ 5018.1537 & 4967.5099 & 4916.4193 & 4865.0000\end{array}$

5072.9212

5180.7353

6575.7169

6501.92446431 .9927

$6735.0098 \quad 6666.6147$

6004.9611

5937.07325888 .8365

6084.6424

5715.4383

5691.4612

5666.9801

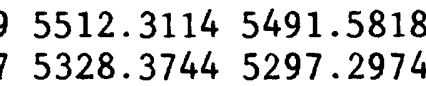

5262.50455238 .5637

5089.61995069 .8808 0.0

$6327.6280 \quad 6250.3642$

5864.52305827 .2298

5628.42595603 .6246

5451.82065431 .5830

5264.94135235 .7099

45099.02735082 .1656

6341.26316249 .2243

5859.71765824 .4249

5637.16985613 .0292 
Table 2.--Listing of data for 1920-70--Continued

Card number

368.

369.

370 .

371.

372.

373.

374.

375 .

376.

377 .

378 .

379 .

380 .

381 .

382 .

383.

384.

385 .

386 .

387.

388 .

389.

390 .

391.

392.

393.

394.

395 .

396.

397.

398.

399.

400 .

401 .

402 .

403.

404.

405.

406.

407.

408.

409 .

410 .

411.

412.

413.

414.

415.

416.

417.
Group III: Array data--Continued Starting head matrix, in feet--Continued
5191.55065168 .9027 5070.8361

0.0 5051.8152
6700.0000

6199.2719

5780.9953

5606.0506

5427.2885

5176.7927

5061.6583

0.0

6201.8745

5782.4999

5613.5434

5430.1737

5156.3502

5042.4201

0.0

6206.3807

6146.3037

5015.7513

5131.5006511

4964.9281

$\begin{array}{ll}5116.6819 & 5104.9217 \\ 4914.0444 & 4860.0000\end{array}$

6659.0754

6576.6003

6509.91916447 .8623

6090.0587

6010.9223

5761.6938
5583.7841

5404.2257

5561.1332

5718.0105

5936.127158

5694.610956

5153.3156

5381.6052

5538.9714

5518.0713

5888.7266

669.2137

5356.4702

5328.2693

5499.3649

5039.2681

5132.6408

4115.4145

5101.9205

5091.7642

4860.0000

6660.0000

6651.0550

6581.3888

6511.3245

6459.2680

5762.84496

6080.6468

5996.0949

5923.76565875 .8000

5591.1433

5741.5690

5720.2951

5698.3590

5675.6675

5521.23105501 .3986

5383.7172

5355.2522

5323.5365

5285.9946

5134.3031

5111.8767

5094.6990

5082.1770

5073.1811
4850.0000

6630.0000

6628.9907

4949.3902

4904.6254
65350.0000

6069.9527

6582.6568

6534.1143

5867.3637

$5783.2740 \quad 5761.8385$

5619.66845597 .0025

5742.1093

5721.9273

5701.5241

5570.5086

5426.9231

5142.2253

5404.9848

5380.1286

5545.6435

5021.6190

5002.5566

0.0

0.0

6211.01076133 .7290

5783.03215762 .2254

5622.21825598 .6428

5421.13225397 .1105

5146.19355119 .1621

4998.7055

4979.3418

0.0

0.0

6207.97176129 .3753

5782.28815761 .8392

5620.65465596 .6788

5416.21645391 .8187

5154.44465129 .6677

$4983.6140 \quad 4969.3450$

$$
0.0
$$

0.0

5095.2401

4972.7776

6615.0000

6048.0282

5741.8056

5570.9141

5372.3770

5093.1146

4953.3518

6630.0000

6030.1578

5740.1252

5571.0790

5367.9086

5101.4281

4949.7528

0.0

5349.7791

5079.7208

4934.5111

6569.3801

$5722.4858 \quad 5704.2460$
5684.3793

$\begin{array}{llll}5544.8878 & 5520.6765 & 5496.332\end{array}$

5344.56245309 .30145266 .8484

5076.10505058 .61735041 .6238

$4922.6780 \quad 4897.1571 \quad 4860.0000$

$6539.1421 \quad 6479.5632 \quad 6432.6319$

5963.40645904 .20985865 .1451

5721.12165706 .56675688 .2325

5544.50725518 .05765492 .2981

5341.21015306 .98965267 .1833

5073.7847

4926.3516

5049.2501

5027.8311

4870.0000

$6485.0000 \quad 6414.3213 \quad 6346.8027$

6195.03996125 .4794

$5782.9806 \quad 5762.4077$

6040.0520

5959.6370

6346.8027
5866.6453

5705.40985687 .1441

5617.94465595 .5321

5740.6624

5721.5059

5516.0153

5488.3897

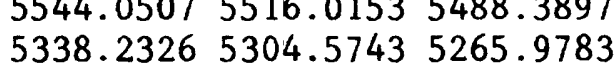

5362.9880

5165.80395140 .9591

5112.2651

5080.4350

5051.5166

5026.4361

4930.0000

6475.00006400 .00876325 .0210

0.0

6180.04006118 .95046047 .1518

5786.12265763 .5644

5742.8620

5960.6842

5866.0440

5706.2372

5686.5714

5621.01135596 .6615

5570.6163

5724.8088

5512.65465483 .2489

5333.10795296 .50755262 .3382

$5176.0285 \quad 5147.5312$

5360.6232

5087.2622

5055.83055031 .7986

0.0

6354.43546254 .0028

5849.30355812 .3304

5645.94515626 .6426

5477.04465451 .4145

5252.89105211 .1574

5083.67455076 .1862

0.0

6378.04736269 .8471

5839.53995808 .8060

$5653.1970 \quad 5632.8755$

5478.83645453 .3251

$5240.6958 \quad 5192.7658$

5065.23625058 .7369

$$
0.0
$$

6384.82036258 .1163

5836.96225809 .3720

$5659.3508 \quad 5638.9122$

5476.81045450 .8099

5227.60445177 .9434

5043.01225034 .5365 0.0

$6393.7734 \quad 6284.0569$

5835.57665807 .6846

5663.47275642 .4739

5470.77135445 .3743

5221.84815179 .3740

5023.82165011 .5368

0.0

6355.97176272 .6431

5833.35685805 .1126

$5666.7919 \quad 5644.0889$

5466.66165441 .2045

5224.95015184 .8366

5006.50434995 .9925

$$
0.0
$$

6299.01936253 .0788

5833.51165804 .5874

5668.29835643 .7989

5462.65945436 .9956

5228.97995194 .0596 
Table 2.--Listing of data for 1920-70--Continued

Card

number
Group III: Array data--Continued

Starting head matrix, in feet--Continued

418.

419 .

420 .

421 .

422 .

423.

424.

425 .

426 .

427.

428 .

429 .

430 .

431 .

432.

433.

434.

435.

436.

437.

438 .

439 .

440 .

441 .

442.

443.

444.

445 .

446 .

447.

448 .

449 .

450 .

451 .

452.

453.

454 .

455 .

456 .

457 .

458 .

459 .

460 .

461 .

462.

463.

464.

465.

466.

467.

$\begin{array}{ccc}4994.3642 & 4991.0568 & 4988.9975 \\ 0.0 & 0.0 & 0.0\end{array}$

4979.73445007 .05134950 .0000

6480.00006417 .34346362 .0945

$6155.00416100 .48736047 .1728 \quad 5964.0180 \quad 5901.5773 \quad 5866.8062$

$5789.89375766 .9778 \quad 5747.67615728 .0423 \quad 5707.72265686 .6629$

5624.96025599 .88175569 .2626

5404.49155379 .86395358 .4429

5181.88345152 .80275122 .2767

5056.07975051 .76195048 .9575

$$
\begin{array}{lll}
0.0 & 0.0 & 0.0
\end{array}
$$

5536.5718

5329.9365

5091.3838

5033.1303

6515.0000

6144.99526087 .87046035 .7384

5798.44835775 .35475754 .0363

59

5732.8588

5625.34395599 .8300

5567.7869

5533.1383

5356.8760

5327.2628

$5185.76885156 .6565 \quad 5124.4085$

5122.40475097 .51085084 .2871

5088.2934

0.0

0.0

6655.0000

5063.0479

$6153.3766 \quad 6069.9490$

6016.6529

6589.8128

5963.7989

$5805.2708 \quad 5783.1762$

$5622.4108 \quad 5596.4315$

5403.27295378 .8073

5760.8769

5739.0323

5566.68765533 .2628

5356.4950

5327.7485

5127.8663

5091.7661

5194.28455163 .8509

5104.1847

5082.911

6740.0000

6637.7440

$6164.2805 \quad 6057.8891$

5810.17535790 .1220

6010.0285

5969.9852

5765.0671

5744.9641

5620.87945595 .8146

5567.8488

5538.9646

5405.10625381 .1128

5355.15305324 .4303

5202.18495176 .901851

$5149.1528 \quad 5143.5496$

0.0

0.0

5142.826

5101.8160

587.30385103 .1228

6850.0000

6190.00796085 .9659602

$5810.4698 \quad 5788.331157$

5620.02745599 .892355

5405.47245384 .3521535

5206.5187
5160.4431

0.0

0.0

6020.9365

6671.3816

5764.69275741 .4674

5569.9191

554

155.3621

5142.246

6890.0000

6215.89646109 .96996034 .2885

5808.93245784 .71755760 .6347

5616.33115591 .05915562 .6741

5403.37575382 .7073

5354.2721

5209.15375190 .7083

5163.5607

5166.0363

5161.3012

5149.8477

7140.00006884 .8206

6223.4856

6117.8257

6042.6312

6708.3498

5989.9090

5805.48515780 .94685756 .82845733 .6504

$5609.84935582 .00325548 .5773 \quad 5512.230$

5393.96135375 .41215349 .10695323 .1776

5211.22395194 .04855167 .35665136 .9995

5170.8249
5166.3973
5156.7291
5025.0000
5505.51395481 .7959

5293.52665262 .6936

.2669

6457.3896

4975.0000

6393.1763

5870.9954

5710.68965686 .5616

5503.05135480 .1055

5293.44515266 .7837

$5064.2720 \quad 5057.0283$

5034.75835000 .0000

6490.9521

6431.0510

5913.43095875 .0835

5715.24715691 .3005

5502.70265478 .8294

5295.22995268 .7247

5069.8997

5052.7079

5065.1021

5920.06175890 .1174

$5719.9770 \quad 5694.9883$

5505.74345477 .2085

5295.26375272 .1239

5078.9225

5071.4613

5070.64415040 .0000

$6548.0310 \quad 6477.4853$
6

6477.4853
5884.9392

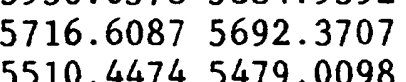

0.0

6291.43836224 .9024

5837.93365814 .1325

5665.48485644 .9026

5457.45995431 .8284

5237.57335210 .2593

5028.57645051 .1937

0.0

6328.78586240 .6588

5845.13095821 .6029

5663.87875644 .7743

5455.88345430 .4039

5241.79965213 .7378

5051.65485095 .5589 0.0

$6360.6566 \quad 6269.6055$

5852.42645828 .0232

5667.54745645 .0381

5454.65515429 .6789

5245.30465220 .2773

5073.10805108 .2865 0.0

$6384.4961 \quad 6289.5449$

5865.05665835 .1543

5670.16715645 .8012

5453.61245429 .4274

5249.10575226 .3917

$5094.7498 \quad 5129.9341$ 0.0

6399.05016301 .1095 $5852.4723 \quad 5832.6644$ 5669.98235645 .0011 $5452.5110 \quad 5428.1535$ 5251.97725228 .2589 5124.85815153 .1861 0.0

6402.80946319 .1265

5848.00165830 .1174

$5665.9550 \quad 5641.5165$

$5443.3898 \quad 5420.3325$

5249.63445227 .1465

5145.35865165 .7530 0.0

6415.03656325 .6318

5845.24315826 .8022

5661.83085637 .1506

5423.98925406 .3627

5248.78135227 .8034

5158.24665173 .5683 0.0 
Table 2.--Listing of data for 1920-70--Continued

Card

number

468.

469.

470.

471 .

472.

473.

474.

475 .

476 .

477.

478 .

479.

480 .

481 .

482.

483.

484.

485.

486 .

487.

488 .

489.

490.

491.

492.

493.

494.

495.

496.

497.

498.

499.

500 .

501 .

502.

503.

504.

505.

506.

507.

508.

509.

510 .

511.

512.

513.

514.

515 .

516.

517 .
Group III: Array data--Continued Starting head matrix, in feet--Continued
0.0 6206.6285

5801.6682

5599.2408

5380.2103

5212.6350519

5175.9244

0.0

6176.4986

5798.4330

5583.5707

5363.9658

5215.7466

5182.7814

0.0

6145.2509

5795.9287

5563.8983

5347.5907

5218.49395

5191.0972518

0.0

6095.2219605

5792.9543576

5539.7625

5335.0012

5219.3719

5205.0091
0.0

6030.8011

5789.8938

5507.4483

5321.5430

5218.6700

5214.3990

0.0

5972.02175956 .9697

5789.50825749 .0895

5477.80765448 .00745425 .66845685 .2822

$5305.22625290 .1355 \quad 5279.6505$

5217.02125200 .84045187 .0125

$5220.8430 \quad 521$

0.0

5210.36475194 .2817

6870.00006670 .1010

5921.92525912 .70455913 .9489

5781.00575738 .00955698 .2448

$5446.5588 \quad 5423.67645401 .7561$

$5286.9407 \quad 5275.2446 \quad 5266.8271$

5214.76185203 .09545190 .4549

5226.0472

0.0 $\begin{array}{ll}5214.5041 & 5196.2949 \\ 6800.0000 & 6617.5166\end{array}$
6710.50236582 .4056

5994.88625922 .6716

5729.05435704 .3873

5476.56865438 .2948

5317.5575

5294.0993

5134.6802

5146.7415
5148.1458
5123.6027

6697.2159

6581.1487

5911.1803

5723.65975697 .8002

5443.2937

5405.7568

5309.38475288 .0747

5155.9300

5148.3064

5155.54065133 .3116
6680.9184
6566.2253

5979.3820

5902.2762

5717.1918

5689.8911

5426.19275392 .2277

5302.5579

5285.2660

5165.2381

5161.2828

(2)

$\begin{array}{llll}6643.8568 & 6535.2881 & 6427.3694\end{array}$

5969.7660

5903.739051

6505.0148

5867.5445

5679.0258

5411.9517

5269.8627

5142.6627

5080.0000

6511.0981

5865.2748

5386.9885

5268.2298

5155.0325

5090.0000

6492.6317

5863.3201

5661.3450

5376.4846

5269.5842

164.3642

327.3694
565.2246

865.2246
650.8272

8709.3386

5680.9963

538

5294.8279

66575368.7143

5280.21945268 .6630

172.16505170 .0124

5171.5262

120.0000

$594.3986 \quad 6470.7503$

948.8856

5901.9556

6348.9914

$5868: 3664$

5632.2286

5408.4859

5378.2000

5359.7166

5282.2126

5272.1148

5262.5266

5176.8327

5177.0482

5176.2680

5157.4629

5125.0000

6377.46786266 .9525

5894.10885871 .8962

25643.50185593 .6321

5397.1689

5371.3296

5349.6198

5268.8232

5261.2339

5251.5228

5181.6663

5180.7906

5181.4208

5183.55035165 .49565140 .0000

6475.9019

6323.3127

6232.4865

5910.5195

5892.1422

5873.6369

5657.16125611 .32635564 .9729

5377.44405355 .04465333 .0364

5254.45415243 .91125233 .0339

5184.12535183 .84385185 .9066

5187.07085171 .52215150 .0000

6416.8610

6276.02106176 .1351

6417.33216315 .2998

5840.97975823 .2755

5653.72625627 .3498

5400.63855388 .1177

5248.54195229 .8951

5165.16775178 .8904 0.0

6401.85856267 .5749

5839.37015820 .4632

$5644.1588 \quad 5614.6974$

5379.24985372 .5742

5251.34225232 .6917

5172.58285185 .4172 0.0

$6353.9108 \quad 6219.4804$

5838.64185819 .0407

5631.72385597 .8468

5366.99275358 .4279

5254.88635237 .3762

5178.53265216 .2236 0.0

6265.41016165 .7838

5841.78435819 .5297

$5615.8440 \quad 5576.0537$

$5357.7440 \quad 5346.7660$

5254.14735236 .9186

5185.30555233 .5771

$$
0.0
$$

6205.05906106 .1397

5847.62445821 .0393

$5583.5030 \quad 5542.3381$

5346.91395335 .1480

5248.57325234 .4715

5190.98795241 .3965

$$
0.0
$$

6147.20966038 .8893

$5851.9928 \quad 5827.5363$

$5544.9671 \quad 5512.5528$

5334.64905320 .5868

5241.11815230 .6463

5196.08935246 .5644 0.0

6110.97365984 .9915

5850.68205820 .6423

5521.17975485 .3889

5315.78495298 .5754

5228.63715222 .4514

5201.48565251 .8408 0.0

6050.87595928 .9516 
Table 2.--Listing of data for 1920-70--Continued

Card

number
Group III: Array data--Continued

Starting head matrix, in feet--Continued
518.

519.

520 .

521 .

522 .

523.

524 .

525 .

526 .

527 .

528 .

529.

530 .

531.

532.

533.

534.

535.

536.

537.

538.

539.

540.

541 .

542.

543.

544.

545.

546.

547.

548.

549.

550 .

551 .

552.

553.

554.

555.

556.

557.

558.

559.

560 .

561 .

562.

563.

564.

565.

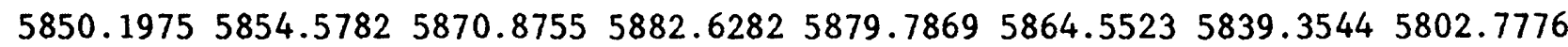
$\begin{array}{llllllll}5755.3476 & 5709.6705 & 5668.7255 & 5623.9681 & 5578.8106 & 5533.9653 & 5490.5295 & 5454.2014\end{array}$ $\begin{array}{llllllll}5418.6747 & 5400.0240 & 5376.0004 & 5354.6793 & 5334.1382 & 5310.1609 & 5288.9571 & 5272.6929\end{array}$ $\begin{array}{llllllll}5262.6643 & 5255.6445 & 5247.3273 & 5234.9764 & 5221.7690 & 5212.6412 & 5213.0125 & 5212.3590\end{array}$

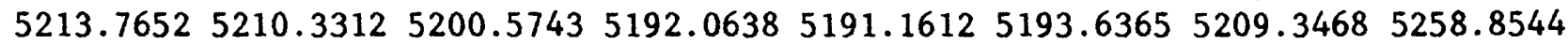
$\begin{array}{lllllll}5230.5953 & 5217.4722 & 5197.6649 & 5188.7290 & 5174.3417 & 5160.0000 & 0.0\end{array}$

$\begin{array}{lllllllll}0.0 & 6700.0000 & 6543.0524 & 6363.7265 & 6213.6823 & 6102.5855 & 5984.0861 & 5866.0045\end{array}$ $5794.25165794 .77235820 .40065853 .0193 \quad 5860.2275 \quad 5847.2522 \quad 5818.94295772 .0504$ $\begin{array}{lllllllll}5697.9205 & 5644.3191 & 5594.1382 & 5557.0543 & 5532.2543 & 5496.0316 & 5462.8040 & 5429.1329\end{array}$ $5398.13755377 .7622 \quad 5350.51145329 .1918 \quad 5305.98745278 .3142 \quad 5254.5225 \quad 5241.7376$ $\begin{array}{lllllll}5233.6521 & 5231.23125223 .6974 & 5213.6297 & 5200.1133 & 5194.9646 & 5199.3392 & 5203.1840\end{array}$ 5211.03735215 .44145214 .49655208 .20585207 .14465209 .53905253 .09525272 .4995 $5235.20485220 .66865199 .11155189 .60395175 .05265150 .0000 \quad 0.0$

$\begin{array}{lllllll}0.0 & 6630.0000 & 6458.40936296 .9711 & 6139.4947 & 6027.0745 & 5912.4797 & 5805.0214\end{array}$ $\begin{array}{lllllll}5760.85445750 .9081 & 5760.5325 & 5802.6781 & 5821.6303 & 5809.8028 & 5778.6391 & 5708.6796\end{array}$ $\begin{array}{lllllllll}5611.1399 & 5561.9038 & 5509.9307 & 5485.4446 & 5470.7477 & 5445.6131 & 5421.8384 & 5392.1137\end{array}$ $5369.31625345 .40575319 .16175302 .6080 \quad 5276.0378 \quad 5246.4002 \quad 5223.3996 \quad 5213.7343$ $5208.13435201 .95585194 .37675188 .24205177 .4577 \quad 5170.05405175 .11295188 .7491$ 5202.01805214 .40885219 .93035219 .54245221 .64455226 .55685251 .66605258 .4568 $\begin{array}{llllllll}5244.8562 & 5225.2003 & 5200.7298 & 5189.7813 & 5175.1148 & 5150.0000 & 0.0\end{array}$

$\begin{array}{llllllll}0.0 & 6550.0000 & 6380.9578 & 6224.5227 & 6074.1922 & 5972.1047 & 5862.8394 & 5765.8495\end{array}$ $\begin{array}{llllllll}5731.3363 & 5723.7205 & 5711.4652 & 5726.9911 & 5732.3626 & 5715.4672 & 5681.5552 & 5615.6552\end{array}$ $\begin{array}{llllllll}5538.5490 & 5496.0509 & 5456.3728 & 5432.5393 & 5405.5674 & 5384.9642 & 5371.3483 & 5349.7924\end{array}$ $5329.02235305 .98885291 .40525275 .2336 \quad 5246.6568 \quad 5220.3184 \quad 5200.64115190 .2483$ $\begin{array}{llllllll}5183.4325 & 5174.0287 & 5166.0437 & 5157.8601 & 5150.6385 & 5140.2322 & 5133.4637 & 5144.8508\end{array}$ 5171.99145196 .88925211 .29745218 .73885226 .55595234 .44575255 .17845260 .4656 $\begin{array}{lllllll}5252.1428 & 5237.7962 & 5209.8306 & 5188.6127 & 5174.5321 & 5150.0000 & 0.0\end{array}$

$\begin{array}{lllllllll}0.0 & 6470.0000 & 6296.0120 & 6139.3125 & 5994.8699 & 5904.6824 & 5803.3893 & 5722.3647\end{array}$ $\begin{array}{lllllllll}5692.9923 & 5682.8328 & 5665.4815 & 5653.8672 & 5634.4014 & 5605.9477 & 5563.7138 & 5512.0535\end{array}$ 5470.05035436 .14035406 .87395384 .36875360 .64205341 .34875326 .41215306 .5525

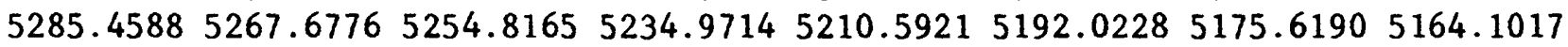
5153.55385143 .43355134 .39055124 .90725118 .16775110 .01745101 .04615097 .4648 $\begin{array}{lllllllll}5120.6647 & 5150.9321 & 5175.8330 & 5199.2580 & 5222.1659 & 5243.6468 & 5259.7666 & 5262.0931\end{array}$ $\begin{array}{lllllll}5252.8685 & 5244.7022 & 5216.5609 & 5188.4114 & 5173.2109 & 5150.0000 & 0.0\end{array}$

$0.0 \quad 6350.00006200 .00006030 .00005900 .00005820 .0000 \quad 5750.0000 \quad 5690.0000$ 5650.00005630 .00005610 .00005590 .00005570 .00005550 .00005510 .00005460 .0000 5430.00005400 .00005375 .00005350 .00005325 .00005305 .00005290 .00005275 .0000 5260.00005235 .00005220 .00005205 .00005185 .00005170 .00005145 .00005130 .0000 $5115.00005105 .00005095 .00005085 .00005080 .0000 \quad 5075.0000 \quad 5070.0000 \quad 5070.0000$ 5070.00005065 .00005090 .00005140 .00005190 .00005245 .00005250 .00005250 .0000

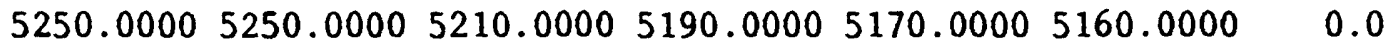

$\begin{array}{llllllll}0.0 & 0.0 & 0.0 & 0.0 & 0.0 & 0.0 & 0.0 & 0.0 \\ 0.0 & 0.0 & 0.0 & 0.0 & 0.0 & 0.0 & 0.0 & 0.0 \\ 0.0 & 0.0 & 0.0 & 0.0 & 0.0 & 0.0 & 0.0 & 0.0 \\ 0.0 & 0.0 & 0.0 & 0.0 & 0.0 & 0.0 & 0.0 & 0.0 \\ 0.0 & 0.0 & 0.0 & 0.0 & 0.0 & 0.0 & 0.0 & 0.0 \\ 0.0 & 0.0 & 0.0 & 0.0 & 0.0 & 0.0 & 0.0 & 0.0 \\ 0.0 & 0.0 & 0.0 & 0.0 & 0.0 & 0.0 & 0.0 & \end{array}$


Card

number

566.

567.

568 .

569.

570.

571.

572.

573.

574.

575.

576.

577.

578.

579 .

580 .

581 .

582.

583.

584 .

585.

586 .

587.

588 .

589.

590.

591.

592.

593.

594.

595.

596.

597.

598.

599.

600.

601 .

602.

603.

604.

605.

606.

607.

608.

609 .

610.

611.

612 .

613.

614.

615 .
Group III: Array data--Continued

Storage coefficient, dimensionless

(First card is parameter card)

1

2

$\begin{array}{rrrrrrrrrrrrrrrrrrrrrr}-1 & -1 & -1 & -1 & -1 & -1 & -1 & -1 & -1 & -1 & -1 & -1 & -1 & -1 & 1 & -1 & & & & & & \\ 0 & & & & & & & & & & \end{array}$

$\begin{array}{llllllllllllllll}1 & 1 & 1 & 1 & 1 & 1 & 1 & 1 & 1 & 1 & 1 & 1 & 1 & 1 & 1 & 1\end{array}$

$\begin{array}{llll}1 & 1 & 1 & 1\end{array}$

$\begin{array}{rlllllllllllllllllll}1 & 1 & 1 & 1 & 1 & 1 & 1 & 1 & -1 & -1 & -1 & -1 & -1 & -1 & -1 & -1 & 1 & 1 & 1 & 1 \\ 1 & 1 & 1 & -1 & -1 & -1 & -1 & -1 & -1 & -1 & -1 & 1 & 1 & 1 & 1 & 1 & -1 & -1 & 1 & 1\end{array}$ $\begin{array}{llllllllllllll}1 & 1 & 1 & -1 & -1 & -1 & -1 & -1 & -1 & -1 & -1 & -1 & -1 & -1\end{array}$

$\begin{array}{llllllllllllllllllll}1 & 1 & 1 & 1 & 1 & 1 & 1 & 1 & 1 & 1 & 1 & 1 & 1 & 1 & 1 & 1 & 1 & 1 & 1 & 1 \\ 1 & 1 & 1 & 1 & 1 & 1 & 1 & 1 & 1 & 1 & 1 & 1 & 1 & -1 & & 1 & 1 & 1 & 1 & 1\end{array}$ $\begin{array}{llllllllllllll}1 & 1 & 1 & 1 & 1 & 1 & 1 & 1 & 1 & 1 & 1 & 1 & 1 & -1\end{array}$

$\begin{array}{lllllllllllllllllll}-1 & 1 & 1 & 1 & 1 & 1 & 1 & 1 & 1 & 1 & 1 & 1 & 1 & 1 & 1 & 1 & 1 & 1 & 1\end{array}$ $\begin{array}{llllllllllllllllllll}1 & 1 & 1 & 1 & 1 & 1 & 1 & 1 & 1 & 1 & 1 & 1 & 1 & 1 & 1 & 1 & 1 & 1 & 1 & 1\end{array}$

$\begin{array}{llllllllllllll}1 & 1 & 1 & 1 & 1 & 1 & 1 & 1 & 1 & 1 & 1 & 1 & 1 & -1\end{array}$

$\begin{array}{lllllllllllllllllll}-1 & 1 & 1 & 1 & 1 & 1 & 1 & 1 & 1 & 1 & 1 & 1 & 1 & 1 & 1 & 1 & 1 & 1 & 1\end{array}$ $\begin{array}{llllllllllllllllllll}1 & 1 & 1 & 1 & 1 & 1 & 1 & 1 & 1 & 1 & 1 & 1 & 1 & 1 & 1 & 1 & 1 & 1 & 1 & 1\end{array}$ $\begin{array}{llllllllllllll}1 & 1 & 1 & 1 & 1 & 1 & 1 & 1 & 1 & 1 & 1 & 1 & 1 & -1\end{array}$ $\begin{array}{lllllllllllllllllll}-1 & 1 & 1 & 1 & 1 & 1 & 1 & 1 & 1 & 1 & 1 & 1 & 1 & 1 & 1 & 1 & 1 & 1 & 1\end{array}$ $\begin{array}{llllllllllllllllllll}1 & 1 & 1 & 1 & 1 & 1 & 1 & 1 & 1 & 1 & 1 & 1 & 1 & 1 & 1 & 1 & 1 & 1 & 1 & 1\end{array}$ $\begin{array}{llllllllllllll}1 & 1 & 1 & 1 & 1 & 1 & 1 & 1 & 1 & 1 & 1 & 1 & 1 & -1\end{array}$ $\begin{array}{lllllllllllll}-1 & 1 & 1 & 1 & 1 & 1 & 1 & 1 & 1 & 1 & 1 & 1 & 1\end{array}$ $\begin{array}{rrrrrrrrrrr:rrr}1 & 1 & 1 & 1 & 1 & 1 & 1 & 1 & 1 & 1 & 1 & 1 & 1 & 1 \\ 1 & 1 & 1 & 1 & 1 & 1 & 1 & 1 & 1 & 1 & 1 & 1 & 1 & -1\end{array}$ $\begin{array}{lllllllllllll}-1 & 1 & 1 & 1 & 1 & 1 & 1 & 1 & 1 & 1 & 1 & 1 & 1\end{array}$ $\begin{array}{rrrrrrrrrrrrrr}1 & 1 & 1 & 1 & 1 & 1 & 1 & 1 & 1 & 1 & 1 & 1 & 1 & 1 \\ 1 & 1 & 1 & 1 & 1 & 1 & 1 & 1 & 1 & 1 & 1 & 1 & 1 & -1\end{array}$ $\begin{array}{rrrrrrrrrrrrrr}1 & 1 & 1 & 1 & 1 & 1 & 1 & 1 & 1 & 1 & 1 & 1 & 1 & -1 \\ & -1 & 1 & 1 & 1 & 1 & 1 & 1 & 1 & 1 & 1 & 1 & 1 & 1\end{array}$ $\begin{array}{rrrrrrrrrrrrrr}1 & 1 & 1 & 1 & 1 & 1 & 1 & 1 & 1 & 1 & 1 & 1 & 1 & 1 \\ 1 & 1 & 1 & 1 & 1 & 1 & 1 & 1 & 1 & 1 & 1 & 1 & 1 & -1\end{array}$ $\begin{array}{rrrrrrrrrrrrrr}1 & 1 & 1 & 1 & 1 & 1 & 1 & 1 & 1 & 1 & 1 & 1 & 1 & 1\end{array}$ $\begin{array}{rrrrrrrrrrrrrr}1 & 1 & 1 & 1 & 1 & 1 & 1 & 1 & 1 & 1 & 1 & 1 & 1 & -1\end{array}$

\begin{tabular}{rrrrrrrrrrr|rrrrrrrrr} 
& -1 & 1 & 1 & 1 & 1 & 1 & 1 & 1 & 1 & 1 & 1 & 1 & 1 & 1 & 1 & 1 & 1 & 1 & 1 \\
1 & 1 & 1 & 1 & 1 & 1 & 1 & 1 & 1 & 1 & 1 & 1 & 1 & 1 & 1 & 1 & 1 & 1 & 1 & 1
\end{tabular} $\begin{array}{llllllllllllll}1 & 1 & 1 & 1 & 1 & 1 & 1 & 1 & 1 & 1 & 1 & 1 & 1 & -1\end{array}$ $\begin{array}{llllll}1 & 1 & 1 & 1 & 1 & 1\end{array}$ $\begin{array}{llllll}1 & 1 & 1 & 1 & 1 & 1\end{array}$ $\begin{array}{llllll}1 & 1 & 1 & 1 & 1 & 1\end{array}$ $\begin{array}{lllll}1 & 1 & 1 & 1 & 1\end{array}$ $\begin{array}{llllll}1 & 1 & 1 & 1 & 1 & 1\end{array}$ $\begin{array}{llllll}1 & 1 & 1 & 1 & 1 & 1\end{array}$ $\begin{array}{llllll}1 & 1 & 1 & 1 & 1 & 1\end{array}$ $\begin{array}{llllllllllllllllllll}1 & 1 & 1 & 1 & 1 & 1 & 1 & 1 & 1 & 1 & 1 & 1 & 1 & 1 & 1 & 1 & 1 & 1 & 1 & 1 \\ 1 & 1 & 1 & 1 & 1 & 1 & 1 & 1 & 1 & 1 & 1 & 1 & 1 & 1 & 1 & 1\end{array}$ $\begin{array}{llllllllllllll}1 & 1 & 1 & 1 & 1 & 1 & 1 & 1 & 1 & 1 & 1 & 1 & 1 & -1\end{array}$

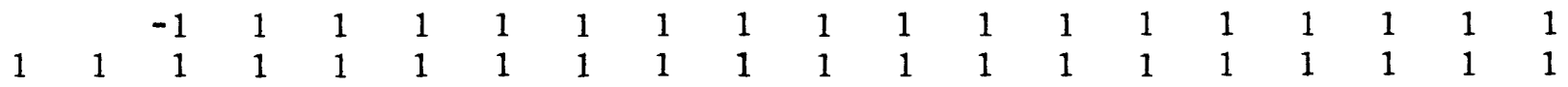
$\begin{array}{llllllllllllll}1 & 1 & 1 & 1 & 1 & 1 & 1 & 1 & 1 & 1 & 1 & 1 & 1 & -1\end{array}$ $\begin{array}{lllllllllllllllllll}-1 & 1 & 1 & 1 & 1 & 1 & 1 & 1 & 1 & 1 & 1 & 1 & 1 & 1 & 1 & 1 & 1 & 1 & 1\end{array}$ $\begin{array}{llllllllllllllllllll}1 & 1 & 1 & 1 & 1 & 1 & 1 & 1 & 1 & 1 & 1 & 1 & 1 & 1 & 1 & 1 & 1 & 1 & 1 & 1\end{array}$

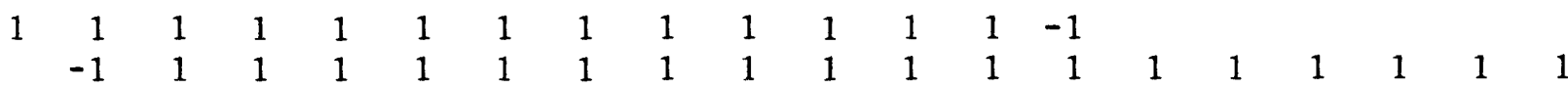


Table 2.--Listing of data for 1920-70--Continued

Card

number

616.

617.

618.

619.

620.

621.

622.

623.

624 .

625 .

626 .

627 .

628.

629.

630.

631.

632.

633.

634.

635.

636.

637.

638.

639.

640.

641.

642.

643.

644.

645.

646.

647.

648.

649.

650.

651 .

652.

653.

654.

655.

656.

657.

658.

659.

660.

661.

662.

663.

664.

665.
Group III: Array data--Continued

Storage coefficient, dimensionless--Continued 
Card number
Group III: Array data--Continued

Storage coefficient, dimensionless--Continued
666.

667.

668.

669.

670.

671.

672.

673.

674.

675.

676.

677.

678 .

679.

680.

681.

682.

683.

684.

685.

686 .

687.

688 .

689.

690.

691.

692.

693.

694.

695.

696.

697.

698.

699.

700.

701 .

702.

703.

704.

705.

706.

707.

708.

709.

710 .

711 .

712 .

713.

714.

715 .

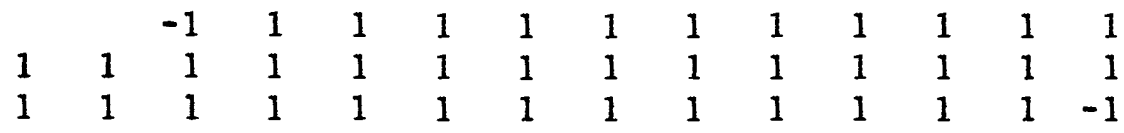

$\begin{array}{llllllllllll}-1 & 1 & 1 & 1 & 1 & 1 & 1 & 1 & 1 & 1 & 1 & 1\end{array}$

$\begin{array}{rrrrrrrrrrrrrr}1 & 1 & 1 & 1 & 1 & 1 & 1 & 1 & 1 & 1 & 1 & 1 & 1 & 1 \\ 1 & 1 & 1 & 1 & 1 & 1 & 1 & 1 & 1 & 1 & 1 & 1 & 1 & -1\end{array}$

$\begin{array}{rlllllllllll}-1 & 1 & 1 & 1 & 1 & 1 & 1 & 1 & 1 & 1 & 1 & 1\end{array}$

$\begin{array}{llllllllllllll}1 & 1 & 1 & 1 & 1 & 1 & 1 & 1 & 1 & 1 & 1 & 1 & 1 & 1\end{array}$

$\begin{array}{llllllllllllll}1 & 1 & 1 & 1 & 1 & 1 & 1 & 1 & 1 & 1 & 1 & 1 & 1 & -1\end{array}$

$\begin{array}{rrrrrrrrrrrrrrrrrrrr}1 & 1 & -1 & 1 & 1 & 1 & 1 & 1 & 1 & 1 & 1 & 1 & 1 & 1 & 1 & 1 & 1 & 1 & 1 & 1 \\ 1 & 1 & 1 & 1 & 1 & 1 & 1 & 1 & 1 & 1 & 1 & 1 & 1 & 1 & 1 & 1 & 1 & 1 & 1 & 1\end{array}$

$\begin{array}{llllllllllllll}1 & 1 & 1 & 1 & 1 & 1 & 1 & 1 & 1 & 1 & 1 & 1 & 1 & -1\end{array}$

$\begin{array}{lllllllllllll}-1 & 1 & 1 & 1 & 1 & 1 & 1 & 1 & 1 & 1 & 1 & 1 & 1\end{array}$

$\begin{array}{llllllllllllll}1 & 1 & 1 & 1 & 1 & 1 & 1 & 1 & 1 & 1 & 1 & 1 & 1 & 1\end{array}$

$\begin{array}{llllllllllllll}1 & 1 & 1 & 1 & 1 & 1 & 1 & 1 & 1 & 1 & 1 & 1 & 1 & -1\end{array}$

$\begin{array}{rllllllllllll}-1 & 1 & 1 & 1 & 1 & 1 & 1 & 1 & 1 & 1 & 1 & 1 & 1 \\ 1 & 1 & 1 & 1 & 1 & 1 & 1 & 1 & 1 & 1 & 1 & 1 & 1\end{array}$

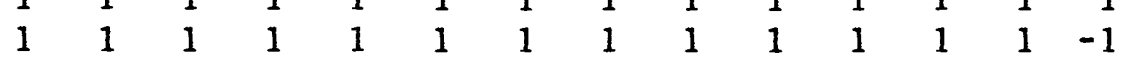

$\begin{array}{lllllllllllll}-1 & 1 & 1 & 1 & 1 & 1 & 1 & 1 & 1 & 1 & 1 & 1 & 1\end{array}$

$\begin{array}{rrrrrrrrrrrrrr}1 & 1 & 1 & 1 & 1 & 1 & 1 & 1 & 1 & 1 & 1 & 1 & 1 & 1 \\ 1 & 1 & 1 & 1 & 1 & 1 & 1 & 1 & 1 & 1 & 1 & 1 & 1 & -1\end{array}$

$\begin{array}{rrrrrrrrrrrrr}-1 & 1 & 1 & 1 & 1 & 1 & 1 & 1 & 1 & 1 & 1 & 1 & 1\end{array}$

$\begin{array}{llllllllllllll}1 & 1 & 1 & 1 & 1 & 1 & 1 & 1 & 1 & 1 & 1 & 1 & 1 & 1\end{array}$

$\begin{array}{llllllllllllll}1 & 1 & 1 & 1 & 1 & 1 & 1 & 1 & 1 & 1 & 1 & 1 & 1 & -1\end{array}$

$\begin{array}{lllllllllllll}-1 & 1 & 1 & 1 & 1 & 1 & 1 & 1 & 1 & 1 & 1 & 1 & 1\end{array}$

$\begin{array}{llllllllllllll}1 & 1 & 1 & 1 & 1 & 1 & 1 & 1 & 1 & 1 & 1 & 1 & 1 & 1\end{array}$

$\begin{array}{llllllllllllll}1 & 1 & 1 & 1 & 1 & 1 & 1 & 1 & 1 & 1 & 1 & 1 & 1 & -1\end{array}$

$\begin{array}{rrrrrrrrrrrrr}-1 & 1 & 1 & 1 & 1 & 1 & 1 & 1 & 1 & 1 & 1 & 1 & 1\end{array}$

$\begin{array}{llllllllllllll}1 & 1 & 1 & 1 & 1 & 1 & 1 & 1 & 1 & 1 & 1 & 1 & 1 & 1\end{array}$

$\begin{array}{llllllllllllll}1 & 1 & 1 & 1 & 1 & 1 & 1 & 1 & 1 & 1 & 1 & 1 & 1 & -1\end{array}$

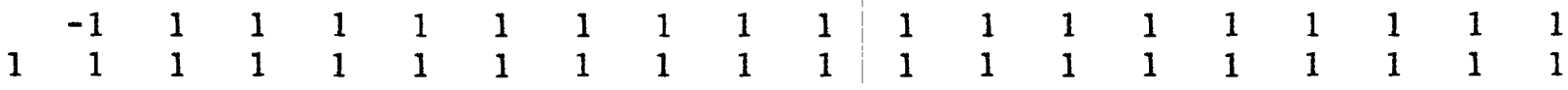

$\begin{array}{lllllllllllllll}1 & 1 & 1 & 1 & 1 & 1 & 1 & 1 & 1 & 1 & 1 & 1 & 1 & -1\end{array}$

$\begin{array}{lllllllllllllllllll}-1 & 1 & 1 & 1 & 1 & 1 & 1 & 1 & 1 & 1 & 1 & 1 & 1 & 1 & 1 & 1 & 1 & 1 & 1\end{array}$

$\begin{array}{llllllllllllllllllll}1 & 1 & 1 & 1 & 1 & 1 & 1 & 1 & 1 & 1 & 1 & 1 & 1 & 1 & 1 & 1 & 1 & 1 & 1 & 1\end{array}$

$\begin{array}{llllllllllllll}1 & 1 & 1 & 1 & 1 & 1 & 1 & 1 & 1 & 1 & 1 & 1 & 1 & -1\end{array}$

$\begin{array}{lllllllllllllllllll}-1 & 1 & 1 & 1 & 1 & 1 & 1 & 1 & 1 & 1 & 1 & 1 & 1 & 1 & 1 & 1 & 1 & 1 & 1\end{array}$

$\begin{array}{llllllllllllllllllll}1 & 1 & 1 & 1 & 1 & 1 & 1 & 1 & 1 & 1 & 1 & 1 & 1 & 1 & 1 & 1 & 1 & 1 & 1 & 1\end{array}$

$\begin{array}{llllllllllllll}1 & 1 & 1 & 1 & 1 & 1 & 1 & 1 & 1 & 1 & 1 & 1 & 1 & -1\end{array}$

$\begin{array}{lllllllllllllllllll}-1 & 1 & 1 & 1 & 1 & 1 & 1 & 1 & 1 & 1 & 1 & 1 & 1 & 1 & 1 & 1 & 1 & 1 & 1\end{array}$

$\begin{array}{llllllllllllllllllll}1 & 1 & 1 & 1 & 1 & 1 & 1 & 1 & 1 & 1 & 1 & 1 & 1 & 1 & 1 & 1 & 1 & 1 & 1 & 1\end{array}$

$\begin{array}{llllllllllllll}1 & 1 & 1 & 1 & 1 & 1 & 1 & 1 & 1 & 1 & 1 & 1 & 1 & -1\end{array}$

$\begin{array}{lllllllllllllllllll}-1 & 1 & 1 & 1 & 1 & 1 & 1 & 1 & 1 & 1 & 1 & 1 & 1 & 1 & 1 & 1 & 1 & 1 & 1\end{array}$

$\begin{array}{llllllllllllllllllll}1 & 1 & 1 & 1 & 1 & 1 & 1 & 1 & 1 & 1 & 1 & 1 & 1 & 1 & 1 & 1 & 1 & 1 & 1 & 1\end{array}$

$\begin{array}{llllllllllllll}1 & 1 & 1 & 1 & 1 & 1 & 1 & 1 & 1 & 1 & 1 & 1 & 1 & -1\end{array}$

$\begin{array}{lllllllllllllllllll}-1 & 1 & 1 & 1 & 1 & 1 & 1 & 1 & 1 & 1 & 1 & 1 & 1 & 1 & 1 & 1 & 1 & 1 & 1\end{array}$

$\begin{array}{llllllllllllllllllll}1 & 1 & 1 & 1 & 1 & 1 & 1 & 1 & 1 & 1 & 1 & 1 & 1 & 1 & 1 & 1 & 1 & 1 & 1 & 1\end{array}$

$\begin{array}{llllllllllllll}1 & 1 & 1 & 1 & 1 & 1 & 1 & 1 & 1 & 1 & 1 & 1 & 1 & -1\end{array}$

$\begin{array}{rllllllllllllllllll}-1 & 1 & 1 & 1 & 1 & 1 & 1 & 1 & 1 & 1 & 1 & 1 & 1 & 1 & 1 & 1 & 1 & 1 & 1 \\ 1 & 1 & 1 & 1 & 1 & 1 & 1 & 1 & 1 & 1 & 1 & 1 & 1 & 1 & 1 & 1 & 1 & 1 & 1\end{array}$ 
Table 2.--Listing of data for 1920-70--Continued

Card

number

716.

717.

718.

719.

720 .

721.

722 .
Group III: Array data--Continued

Storage coefficient, dimensionless--Continued

$$
\begin{array}{rrrrrrrrrrrrrrrrrrrrr}
1 & 1 & 1 & 1 & 1 & 1 & 1 & 1 & 1 & 1 & 1 & 1 & 1 & -1 & & & & & & \\
& -1 & -1 & -1 & -1 & -1 & -1 & -1 & -1 & -1 & -1 & -1 & -1 & -1 & -1 & -1 & -1 & -1 & -1 & -1 \\
-1 & -1 & -1 & -1 & -1 & -1 & -1 & -1 & -1 & -1 & -1 & -1 & -1 & -1 & -1 & -1 & -1 & -1 & -1 & -1 \\
-1 & -1 & -1 & -1 & -1 & -1 & -1 & -1 & -1 & -1 & -1 & -1 & -1 & -1 & & & & & &
\end{array}
$$

Group III: Array data--Continued

Hydraulic conductivity, in feet per second

(First card is parameter card)

723.

724.

$$
\text { 1.E-06 }
$$

0

1

2

0

0

725.

726.

727.

728.

729.

730.

731.

732.

733.

734.

735.

736.

737.

738.

739.

740 .

741 .

742 .

743.

744.

745 .

746.

747.

748 .

749 .

750.

$$
0
$$

0

0

$\begin{array}{llllllllllllllll}20 & 30 & 100 & 100 & 100 & 100 & 100 & 80 & 80 & 20 & 20 & 10 & 10 & 30 & 80 & 100\end{array}$ $\begin{array}{llll}200 & 200 & 1 & 60\end{array}$

751.

0

$\begin{array}{llllllllllllllll}20 & 20 & 50 & 50 & 50 & 20 & 10 & 10 & 10 & 10 & 10 & 10 & 10 & 30 & 30 & 30 \\ 10 & 10 & 10 & 20 & & & & & & & & & & & & \end{array}$

0

$\begin{array}{lllllllll}20 & 20 & 10 & 10 & 20 & 20 & 10 & 10\end{array}$

$10 \quad 40$

$\begin{array}{llllll}2 & 2 & 2 & 20 & 20 & 20\end{array}$

$\begin{array}{llllll}20 & 20 & 10 & 10 & 30 & 20\end{array}$

0

$\begin{array}{llllll}30 & 30 & 30 & 10 & 10 & 10\end{array}$

$\begin{array}{llllll}60 & 60 & 100 & 100 & 100 & 80\end{array}$

$\begin{array}{llllll}10 & 10 & 10 & 10 & 20 & 20\end{array}$

$\begin{array}{lllll}10 & 40 & 5 & 10 & 10\end{array}$

$\begin{array}{llllll}30 & 40 & 20 & 10 & 10 & 10\end{array}$

$\begin{array}{llllll}60 & 60 & 400 & 400 & 400 & 300\end{array}$

$\begin{array}{llllll}10 & 20 & 20 & 10 & 10 & 20\end{array}$

$\begin{array}{lllll}10 & 30 & 10 & 5 & 10\end{array}$

$\begin{array}{llllll}10 & 10 & 10 & 10 & 10 & 10\end{array}$

$\begin{array}{rrrrrr}200 & 200 & 400 & 600 & 600 & 600\end{array}$

752 .

10

753.

754.

755.

756.

757.

758.

759 .

20

100

100

$\begin{array}{rrrrr}50 & 50 & 10 & 5 & 10\end{array}$

$\begin{array}{llllll}2 & 5 & 10 & 10 & 10 & 10\end{array}$

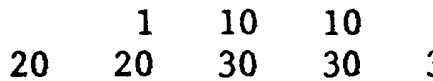

$\begin{array}{lll}50 & 40 & 40\end{array}$

$\begin{array}{lll}60 & 60 & 20\end{array}$
55

$\begin{array}{rrr}30 & 30 & 30 \\ 10 & 10 & 5\end{array}$ $\begin{array}{rrrrrrrrrr}5 & 5 & 5 & 2 & 2 & 900 & 20 & 10 & 10 & 10\end{array}$

$\begin{array}{rrrrrrrrr} & 1 & 5 & 70 & 70 & 60 & 50 & 30 & 30\end{array}$

$\begin{array}{rrrrrrrrrr}60 & 20 & 10 & 5 & 800 & 800 & 10 & 5 & 5 & 10\end{array}$

$\begin{array}{llllllllll}10 & 20 & 20 & 60 & 70 & 60 & 80 & 80 & 70 & 30\end{array}$ $\begin{array}{llllllllll}10 & 10 & 20 & 20 & 30 & 50 & 10 & 5 & 10 & 60\end{array}$

$\begin{array}{llllllllll}10 & 10 & 20 & 50 & 40 & 60 & 70 & 70 & 50 & 30\end{array}$ $\begin{array}{llllllllll}10 & 20 & 30 & 30 & 60 & 2 & 2 & 5 & 10 & 60\end{array}$

$\begin{array}{llllllllll}10 & 10 & 10 & 30 & 30 & 30 & 50 & 30 & 20 & 10\end{array}$ $\begin{array}{rrrrrrrrrr}30 & 40 & 50 & 30 & 10 & 2 & 2 & 5 & 10 & 100\end{array}$ $\begin{array}{llllllllll}30 & 30 & 30 & 20 & 20 & 10 & 10 & 5 & 2 & 2\end{array}$

$\begin{array}{llllllllll}5 & 5 & 20 & 30 & 20 & 20 & 50 & 20 & 10 & 20\end{array}$ $\begin{array}{llllllllll}40 & 40 & 20 & 20 & 10 & 10 & 20 & 40 & 40 & 50\end{array}$ $\begin{array}{rrrrrrrrrr}4 & 2 & 5 & 10 & 10 & 10 & 10 & 2 & 5 & 20\end{array}$ 
Table 2.--Listing of data for 1920-70--Continued

Card

number

760 .

761.

762.

763.

764.

765 .

766.

767.

768.

769.

770 .

771.

772.

773.

774.

775.

776.

777.

778.

779.

780 .

781 .

782.

783.

784.

785 .

786 .

787.

788.

789.

790.

791.

792.

793.

794.

795.

796.

797.

798.

799.

800 .

801 .

802 .

803.

804.

805.

806.

807 .

808 .
Group III: Array data--Continued

Hydraulic conductivity, in feet per second--Continued $\begin{array}{rrrrrrrrrrrrrrrr} & 1 & 5 & 5 & 5 & 5 & 10 & 10 & 30 & 20 & 10 & 10 & 10 & 10 & 10 & 20 \\ 20 & 20 & 10 & 10 & 20 & 20 & 20 & 20 & 20 & 20 & 20 & 20 & 20 & 20 & 20 & 40\end{array}$

$\begin{array}{llllllllllllllll}40 & 20 & 20 & 10 & 10 & 1 & 1 & 1 & 5 & 10 & 10 & 10 & 10 & 10 & 20 & 50\end{array}$

$\begin{array}{llllll}80 & 80 & 40 & 40 & 20 & 20\end{array}$

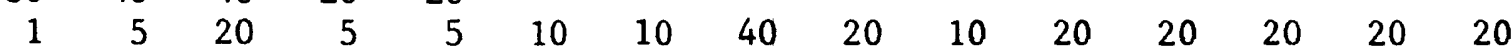

$\begin{array}{llllllllllllllll}20 & 20 & 10 & 10 & 20 & 20 & 20 & 20 & 20 & 20 & 10 & 20 & 30 & 40 & 30 & 30\end{array}$

$\begin{array}{llllllllllllllll}10 & 10 & 5 & 5 & 1 & 1 & 1 & 2 & 5 & 5 & 5 & 5 & 10 & 10 & 20 & 40\end{array}$

$\begin{array}{llllll}70 & 50 & 50 & 60 & 30 & 30\end{array}$

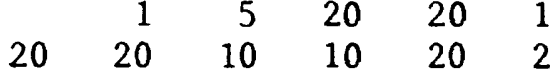

$\begin{array}{llllll}40 & 40 & 40 & 40 & 30 & 30\end{array}$

$\begin{array}{llllll}60 & 50 & 10 & 60 & 80 & 80\end{array}$

$\begin{array}{llllllllll}30 & 10 & 10 & 5 & 5 & 10 & 10 & 10 & 10 & 5\end{array}$

$\begin{array}{llllllllll}20 & 20 & 40 & 40 & 40 & 40 & 40 & 40 & 30 & 30\end{array}$

$\begin{array}{lllllllllll}30 & 30 & 30 & 50 & 50 & 50 & 70 & 70 & 80 & 80 & 80\end{array}$

$\begin{array}{llllllllllllll}10 & 20 & 30 & 30 & 30 & 5 & 5 & 5 & 10 & 10 & 10 & 10 & 10 & 10\end{array}$

$\begin{array}{llllllllllllllll}10 & 10 & 10 & 10 & 20 & 20 & 30 & 40 & 60 & 60 & 60 & 60 & 60 & 60 & 60 & 50\end{array}$

$\begin{array}{llllllllllllllll}50 & 50 & 50 & 40 & 40 & 40 & 40 & 40 & 50 & 50 & 60 & 70 & 80 & 80 & 80 & 60\end{array}$

$\begin{array}{llllll}10 & 10 & 10 & 50 & 90 & 90\end{array}$

$\begin{array}{rrrrrrrrrrrrrr}20 & 20 & 10 & 5 & 10 & 5 & 5 & 5 & 2 & 5 & 5 & 5 & 5 & 5\end{array}$

$\begin{array}{llllllllllllllll}10 & 10 & 10 & 20 & 20 & 30 & 40 & 60 & 60 & 60 & 60 & 60 & 60 & 60 & 60 & 60\end{array}$

$\begin{array}{llllllllllllllll}60 & 50 & 50 & 50 & 50 & 50 & 50 & 50 & 50 & 50 & 80 & 100 & 200 & 400 & 60 & 60\end{array}$

$\begin{array}{llllll}20 & 10 & 10 & 40 & 100 & 500\end{array}$

$\begin{array}{llllllllllllll}20 & 5 & 10 & 10 & 10 & 5 & 5 & 2 & 2 & 2 & 10 & 10 & 10 & 10\end{array}$

$\begin{array}{llllllllllllllll}10 & 10 & 20 & 30 & 30 & 40 & 60 & 60 & 60 & 60 & 60 & 60 & 60 & 60 & 60 & 60\end{array}$

$\begin{array}{llllllllllllllll}60 & 100 & 100 & 100 & 100 & 100 & 100 & 90 & 90 & 80 & 80 & 100 & 100 & 200 & 400 & 400\end{array}$

$\begin{array}{llllll}20 & 20 & 20 & 100 & 500 & 500\end{array}$

$\begin{array}{llllllllllllll}20 & 5 & 5 & 5 & 2 & 2 & 2 & 2 & 2 & 5 & 5 & 10 & 10 & 10\end{array}$

$\begin{array}{llllllllllllllll}10 & 20 & 30 & 30 & 40 & 50 & 60 & 60 & 60 & 60 & 60 & 60 & 60 & 60 & 60 & 60\end{array}$

$\begin{array}{rrrrrrrrrrrrrrrr}100 & 100 & 100 & 200 & 200 & 200 & 200 & 100 & 90 & 90 & 90 & 100 & 100 & 200 & 400 & 400\end{array}$

$\begin{array}{llllll}500 & 500 & 500 & 500 & 500 & 500\end{array}$

$\begin{array}{lllllllllllllll}40 & 10 & 5 & 5 & 5 & 2 & 2 & 2 & 2 & 2 & 2 & 10 & 10 & 10 & 20\end{array}$

$\begin{array}{llllllllllllllll}30 & 30 & 30 & 30 & 40 & 50 & 50 & 60 & 60 & 60 & 60 & 60 & 60 & 60 & 60 & 60\end{array}$

$\begin{array}{llllllllllllllll}100 & 200 & 200 & 200 & 200 & 200 & 200 & 200 & 200 & 400 & 400 & 200 & 200 & 200 & 200 & 400\end{array}$

$500 \quad 500 \quad 500 \quad 500 \quad 500 \quad 500$

$\begin{array}{llrrrrrrrrrrrrr}40 & 2 & 10 & 10 & 5 & 2 & 10 & 5 & 2 & 2 & 10 & 10 & 20 & 30 & 30\end{array}$

$\begin{array}{llllllllllllllll}30 & 30 & 30 & 30 & 40 & 40 & 40 & 40 & 40 & 40 & 40 & 50 & 50 & 80 & 100 & 200\end{array}$

$\begin{array}{llllllllllllllll}200 & 200 & 200 & 200 & 200 & 200 & 200 & 200 & 400 & 400 & 200 & 200 & 200 & 200 & 200 & 400\end{array}$

$500 \quad 500 \quad 500 \quad 500 \quad 500 \quad 500$

$\begin{array}{lllllllllllllll}40 & 10 & 10 & 5 & 5 & 10 & 10 & 10 & 10 & 10 & 10 & 10 & 30 & 30 & 30\end{array}$

$\begin{array}{llllllllllllllll}30 & 20 & 20 & 20 & 20 & 30 & 30 & 30 & 30 & 30 & 50 & 50 & 100 & 200 & 200 & 200\end{array}$

$\begin{array}{llllllllllllllll}200 & 200 & 200 & 200 & 200 & 200 & 200 & 200 & 200 & 200 & 100 & 100 & 100 & 100 & 200 & 400\end{array}$

$400 \quad 400 \quad 400 \quad 300 \quad 400 \quad 400$

$\begin{array}{lllll}20 & 10 & 20 & 10 & 10\end{array}$

$\begin{array}{rrrrrrrrrrrrrrrr}30 & 30 & 50 & 50 & 50 & 50 & 100 & 100 & 100 & 100 & 100 & 200 & 200 & 200 & 200 & 100\end{array}$

$\begin{array}{llllllllllllllll}100 & 100 & 100 & 100 & 80 & 80 & 100 & 100 & 100 & 100 & 100 & 100 & 100 & 100 & 200 & 400\end{array}$

$\begin{array}{llllll}400 & 400 & 300 & 200 & 200 & 400\end{array}$

$\begin{array}{lllllllllllllll}30 & 30 & 10 & 10 & 10 & 10 & 10 & 20 & 20 & 20 & 10 & 20 & 30 & 50 & 50\end{array}$

$\begin{array}{rrrrrrrrrrrrrrrr}50 & 90 & 90 & 90 & 100 & 100 & 100 & 100 & 200 & 200 & 200 & 200 & 200 & 200 & 200 & 100\end{array}$

$\begin{array}{rrrrrrrrrrrrrrrr}100 & 100 & 100 & 80 & 80 & 80 & 80 & 100 & 100 & 100 & 100 & 100 & 100 & 100 & 100 & 100\end{array}$

$\begin{array}{llllll}100 & 90 & 100 & 100 & 100 & 100\end{array}$

$\begin{array}{llrrr}40 & 20 & 10 & 10 & 10\end{array}$

$\begin{array}{lllllllllll}10 & 10 & 20 & 20 & 20 & 20 & 80 & 80 & 50 & 50 & 50\end{array}$ 
Table 2.--Listing of data for 1920-70--Continued

Card

number

809.

810 .

811.

812 .

813.

814.

815 .

816.

817.

818.

819 .

820 .

821 .

822.

823.

824 .

825 .

826 .

827.

828 .

829 .

830 .

831.

832.

833.

834.

835.

836.

837.

838 .

839 .

840 .

841 .

842.

843.

844.

845 .

846 .

847.

848 .

849 .

850 .

851 .

852.

853.

854.

855 .

856.

857.

858.
Group III: Array data--Continued

Hydraulic conductivity, in feet per second--Continued

$\begin{array}{rrrrrrrrrrrrrrrr}50 & 90 & 90 & 100 & 100 & 100 & 100 & 200 & 200 & 100 & 100 & 100 & 100 & 100 & 100 & 100 \\ 100 & 100 & 100 & 90 & 80 & 70 & 50 & 80 & 90 & 90 & 90 & 200 & 100 & 100 & 50 & 40 \\ 20 & 20 & 20 & 20 & 80 & 80 & & & & & & & & & & \\ & 30 & 10 & 10 & 10 & 10 & 5 & 10 & 20 & 20 & 20 & 20 & 30 & 60 & 60 & 60 \\ 70 & 80 & 100 & 100 & 100 & 100 & 100 & 100 & 100 & 100 & 100 & 100 & 100 & 100 & 100 & 100 \\ 100 & 100 & 90 & 80 & 70 & 60 & 50 & 50 & 70 & 90 & 90 & 100 & 100 & 100 & 100 & 80 \\ 60 & 30 & 20 & 20 & 60 & 60 & & & & & & & & & & \\ & 10 & 20 & 30 & 10 & 10 & 10 & 10 & 20 & 20 & 10 & 10 & 20 & 30 & 80 & 70 \\ 80 & 100 & 100 & 100 & 100 & 100 & 100 & 100 & 100 & 100 & 100 & 100 & 100 & 100 & 100 & 100 \\ 90 & 90 & 90 & 70 & 70 & 50 & 50 & 50 & 70 & 80 & 80 & 80 & 100 & 100 & 60 & 60 \\ 50 & 30 & 20 & 20 & 60 & 60 & & & & & & & & & & \\ & 10 & 20 & 30 & 10 & 10 & 5 & 20 & 20 & 20 & 20 & 10 & 20 & 20 & 20 & 20 \\ 100 & 100 & 100 & 100 & 100 & 100 & 200 & 200 & 200 & 200 & 200 & 200 & 200 & 200 & 100 & 90 \\ 90 & 90 & 90 & 70 & 70 & 50 & 50 & 60 & 80 & 90 & 90 & 90 & 90 & 100 & 60 & 60 \\ 20 & 20 & 20 & 20 & 50 & 50 & & & & & & & & & & \\ & 20 & 10 & 10 & 10 & 10 & 5 & 5 & 10 & 10 & 10 & 10 & 20 & 30 & 50 & 60 \\ 100 & 100 & 100 & 100 & 100 & 100 & 100 & 100 & 100 & 60 & 60 & 60 & 60 & 60 & 40 & 40 \\ 40 & 30 & 20 & 20 & 20 & 20 & 20 & 30 & 80 & 80 & 90 & 90 & 90 & 90 & 40 & 20 \\ 20 & 20 & 20 & 20 & 50 & 50 & & & & & & & & & & \end{array}$

$\begin{array}{llllll}20 & 20 & 20 & 20 & 50 & 50\end{array}$ $\begin{array}{llll}400 & 50 & 30 & 5\end{array}$

$\begin{array}{llrrrr}70 & 100 & 100 & 100 & 100 & 100\end{array}$

$\begin{array}{llllll}40 & 40 & 20 & 20 & 20 & 20\end{array}$

$\begin{array}{llllll}20 & 20 & 20 & 20 & 80 & 80\end{array}$

$\begin{array}{llll}10 & 10 & 10\end{array}$

$\begin{array}{llllll}90 & 90 & 100 & 100 & 100 & 10\end{array}$

$\begin{array}{llllll}90 & 90 & 90 & 70 & 60 & 60\end{array}$

$\begin{array}{llllll}20 & 20 & 40 & 90 & 2500 & 3500\end{array}$

$\begin{array}{llll}10 & 10 & 10 & 1\end{array}$

$90 \quad 100 \quad 100 \quad 200 \quad 200 \quad 100$

$\begin{array}{llllll}90 & 90 & 90 & 70 & 60 & 60\end{array}$

$4000 \quad 40003800 \quad 40004000 \quad 20$

$\begin{array}{lll}10 & 10 & 20\end{array}$

$\begin{array}{llllll}90 & 100 & 100 & 200 & 200 & 200\end{array}$

$\begin{array}{llllll}200 & 200 & 200 & 200 & 100 & 100\end{array}$

$5000 \quad 400030003000$

520

$\begin{array}{rrrrrr}100 & 100 & 200 & 200 & 200 & 200\end{array}$

$\begin{array}{llllll}200 & 300 & 300 & 200 & 200 & 300\end{array}$

$\begin{array}{llllll}2300 & 20 & 10 & 10 & 50 & 200\end{array}$

$\begin{array}{rrrrrr}100 & 200 & 200 & 200 & 200 & 200\end{array}$

$200 \quad 300 \quad 300 \quad 200 \quad 200$

400

$\begin{array}{rrrrrr}2 & 5 & 10 & 10 & 100 & 200\end{array}$

$\begin{array}{rrrrrr}100 & 100 & 100 & 100 & 100 & 100\end{array}$

$\begin{array}{llllll}200 & 300 & 300 & 200 & 300 & 400\end{array}$

$\begin{array}{llllll}1 & 10 & 20 & 20 & 80 & 80\end{array}$

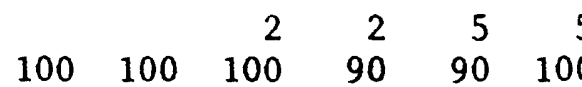

$\begin{array}{llllll}200 & 300 & 300 & 200 & 200 & 300\end{array}$ $\begin{array}{llllllllll}1 & 10 & 10 & 10 & 5 & 10 & 20 & 40 & 60 & 60\end{array}$

$\begin{array}{llllllllll}100 & 100 & 100 & 60 & 60 & 60 & 60 & 60 & 40 & 40\end{array}$ $\begin{array}{llllllllll}20 & 30 & 200 & 200 & 400 & 800 & 600 & 200 & 90 & 20\end{array}$

$\begin{array}{llllllllll}1 & 2 & 2 & 5 & 5 & 10 & 20 & 50 & 60 & 60\end{array}$ $\begin{array}{rrrrrrrrrr}100 & 100 & 100 & 60 & 60 & 80 & 80 & 80 & 80 & 90\end{array}$ $\begin{array}{llllllllll}80 & 100 & 200 & 200 & 600 & 800 & 800 & 800 & 300 & 50\end{array}$

$\begin{array}{llllllllll}1 & 2 & 5 & 2 & 10 & 10 & 20 & 30 & 40 & 60\end{array}$ $\begin{array}{llllllllll}90 & 90 & 80 & 80 & 80 & 80 & 90 & 90 & 90 & 90\end{array}$ $\begin{array}{llllllllll}60 & 90 & 200 & 200 & 200 & 200 & 800 & 800 & 4000 & 4000\end{array}$

$\begin{array}{llllllllll}20 & 5 & 5 & 5 & 5 & 10 & 20 & 30 & 30 & 60\end{array}$ $\begin{array}{rrrrrrrrrr}200 & 100 & 200 & 200 & 200 & 200 & 200 & 200 & 200 & 200\end{array}$ $\begin{array}{llllllllll}100 & 200 & 300 & 300 & 200 & 200 & 300 & 300 & 4000 & 4000\end{array}$

$\begin{array}{llllllllll}30 & 30 & 10 & 10 & 5 & 10 & 20 & 30 & 40 & 90\end{array}$ $\begin{array}{llllllllll}200 & 200 & 200 & 200 & 200 & 200 & 100 & 200 & 200 & 200\end{array}$ $\begin{array}{llllllllll}400 & 300 & 300 & 300 & 300 & 300 & 300 & 700 & 5000 & 5000\end{array}$

$\begin{array}{rrrrrrrrrr}5 & 10 & 10 & 30 & 10 & 10 & 30 & 40 & 80 & 100\end{array}$ $\begin{array}{llllllllll}200 & 200 & 200 & 100 & 100 & 100 & 200 & 200 & 200 & 200\end{array}$ $\begin{array}{llllllllll}400 & 400 & 400 & 400 & 400 & 400 & 300 & 5000 & 5000 & 2\end{array}$ $\begin{array}{rrrrrrrrrr}5 & 5 & 10 & 30 & 10 & 10 & 50 & 70 & 90 & 100\end{array}$ $\begin{array}{llllllllll}200 & 200 & 200 & 100 & 100 & 100 & 200 & 200 & 200 & 200\end{array}$ $\begin{array}{llllllllrr}300 & 300 & 400 & 400 & 400 & 400 & 5000 & 5000 & 30 & 2\end{array}$

$\begin{array}{llllllllll}5 & 5 & 5 & 30 & 30 & 40 & 50 & 70 & 80 & 100\end{array}$ $\begin{array}{rrrrrrrrrr}90 & 100 & 100 & 100 & 100 & 100 & 200 & 200 & 200 & 200\end{array}$ $\begin{array}{llllllllll}300 & 300 & 300 & 200 & 300 & 300 & 2800 & 5000 & 30 & 10\end{array}$ 
Card number

859.

860 .

861 .

862.

863.

864.

865.

866.

867.

868.

869.

870.

871.

872.

873.

874.

875.

876.

877.

878.

879 .

880 .

881 .

882.

883.

884.

885 .

886 .

887.

888 .

889.

890 .

891.

892 .

893.

894.

895.

896.

897.

898.

899.

900 .

901.

902.

903.

904.

905.

906.

907.

908.
Group III: Array data--Continued

Hydraulic conductivity, in feet per second--Continued $\begin{array}{llllll}5 & 10 & 20 & 20 & 70 & 70\end{array}$

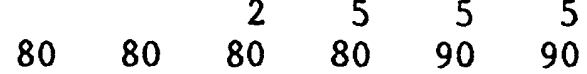

$\begin{array}{llllll}200 & 200 & 200 & 200 & 300 & 300\end{array}$

$\begin{array}{llllll}5 & 10 & 10 & 20 & 70 & 70\end{array}$

$\begin{array}{rrrrrr}90 & 90 & 80 & 80 & 80 & 100\end{array}$

$\begin{array}{llllll}200 & 200 & 100 & 100 & 300 & 300\end{array}$

$\begin{array}{llllll}30 & 30 & 30 & 50 & 70 & 70\end{array}$

$\begin{array}{rrrrrr}80 & 80 & 100 & 100 & 100 & 100\end{array}$

$\begin{array}{rrrrrr}300 & 100 & 100 & 100 & 100 & 100\end{array}$

$\begin{array}{rrrrrr}50 & 30 & 40 & 60 & 70 & 70\end{array}$

$\begin{array}{rrrrr}5 & 10 & 10 & 10 & 10\end{array}$

$\begin{array}{rrrrrr}80 & 80 & 100 & 100 & 100 & 100\end{array}$

$\begin{array}{rrrrrr}300 & 100 & 100 & 100 & 100 & 100\end{array}$

$\begin{array}{llllll}50 & 50 & 60 & 60 & 70 & 70\end{array}$

$$
\begin{array}{rrrrr}
10 & 5 & 5 & 5 & 5
\end{array}
$$

$\begin{array}{rrrrrr}80 & 90 & 100 & 100 & 100 & 100\end{array}$

$\begin{array}{rrrrrr}300 & 100 & 100 & 100 & 100 & 100 \\ 50 & 50 & 60 & 60 & 70 & 70\end{array}$

$\begin{array}{rrrrrr}50 & 50 & 60 & 60 & 70 & 70\end{array}$

$\begin{array}{rrrrr}5 & 2 & 5 & 5 & 5 \\ 90 & 100 & 100 & 100 & 100\end{array}$

$\begin{array}{rrrrrr}90 & 90 & 100 & 100 & 100 & 100 \\ 300 & 100 & 100 & 100 & 100 & 200\end{array}$

$\begin{array}{rrrrrr}30 & 50 & 50 & 60 & 70 & 70\end{array}$

$\begin{array}{rrrrr}5 & 5 & 5 & 5 & \\ 90 & 100 & 100 & 100 & 100\end{array}$

$\begin{array}{rrrrrr}90 & 90 & 100 & 100 & 100 & 100 \\ 500 & 300 & 300 & 200 & 200 & 200\end{array}$

$\begin{array}{llllll}30 & 30 & 40 & 50 & 70 & 70\end{array}$

$\begin{array}{lll}5 & 5 & 5\end{array}$

$\begin{array}{llllll}40 & 60 & 70 & 90 & 90 & 100\end{array}$

$\begin{array}{llllll}500 & 500 & 300 & 200 & 200 & 200\end{array}$

$\begin{array}{llllll}10 & 20 & 40 & 50 & 80 & 80\end{array}$

$\begin{array}{lllll}5 & 10 & 10 & 5 & 2\end{array}$

$\begin{array}{llllll}40 & 50 & 60 & 60 & 60 & 40\end{array}$

$\begin{array}{llllll}600 & 500 & 300 & 200 & 300 & 100\end{array}$

$\begin{array}{llllll}30 & 20 & 40 & 50 & 80 & 80\end{array}$

$\begin{array}{llll}5 & 10 & 10 & 20\end{array}$

$\begin{array}{lllll}10 & 30 & 40 & 30 & 30\end{array}$

600700

$30 \quad 10$

$500 \quad 500$

505

1010

$\begin{array}{llll}10 & 10 & 20 & 30\end{array}$

$\begin{array}{rrrrr}500 & 700 & 700 & 500 & 300\end{array}$

$\begin{array}{lllll}30 & 10 & 70 & 80 & 80\end{array}$

$10 \quad 10 \quad 20 \quad 20$

$\begin{array}{llllll}10 & 10 & 10 & 10 & 30 & 30\end{array}$

$\begin{array}{llllll}300 & 600 & 700 & 700 & 500 & 50\end{array}$

$\begin{array}{rrrrrr}30 & 10 & 80 & 100 & 100 & 90\end{array}$ $\begin{array}{llll}5 & 5 & 5 & 30\end{array}$

$\begin{array}{rrr}50 & 40 & 50\end{array}$

70

70

70

$\begin{array}{llllllllll}90 & 80 & 80 & 90 & 100 & 100 & 100 & 200 & 200 & 200\end{array}$ $\begin{array}{lllllllllll}300 & 300 & 200 & 200 & 100 & 100 & 2900 & 3000 & 0 & 10 & 10\end{array}$

$\begin{array}{rrrrrrrrrr}5 & 5 & 10 & 10 & 30 & 20 & 20 & 20 & 100 & 90\end{array}$ $\begin{array}{rrrrrrrrrr}100 & 100 & 100 & 100 & 100 & 300 & 300 & 300 & 300 & 200\end{array}$ $\begin{array}{llllllllll}200 & 200 & 200 & 200 & 100 & 100 & 3000 & 40 & 5 & 10\end{array}$

$\begin{array}{llllllllll}10 & 10 & 10 & 10 & 20 & 20 & 20 & 30 & 100 & 100\end{array}$ $\begin{array}{llllllllll}100 & 100 & 100 & 100 & 100 & 100 & 300 & 300 & 300 & 300\end{array}$

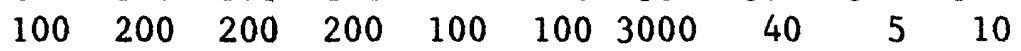

$\begin{array}{llllllllll}10 & 10 & 10 & 10 & 20 & 20 & 20 & 40 & 90 & 90\end{array}$ $\begin{array}{llllllllll}100 & 70 & 60 & 60 & 40 & 40 & 40 & 60 & 300 & 300\end{array}$ $\begin{array}{llllllllll}100 & 200 & 200 & 200 & 100 & 200 & 2000 & 40 & 5 & 10\end{array}$

$\begin{array}{llllllllll}5 & 5 & 5 & 10 & 20 & 10 & 10 & 30 & 90 & 90\end{array}$ $\begin{array}{rrrrrrrrrr}100 & 90 & 80 & 50 & 50 & 60 & 60 & 80 & 100 & 400\end{array}$ $\begin{array}{llllllllll}200 & 200 & 300 & 300 & 200 & 400 & 2000 & 40 & 10 & 10\end{array}$

$\begin{array}{llllllllll}2 & 5 & 10 & 10 & 20 & 10 & 20 & 40 & 90 & 90\end{array}$ $\begin{array}{rrrrrrrrrr}100 & 80 & 80 & 50 & 50 & 90 & 200 & 700 & 200 & 400\end{array}$ $\begin{array}{llllllllll}200 & 200 & 300 & 200 & 100 & 400 & 1000 & 70 & 10 & 10\end{array}$

$\begin{array}{rrrrrrrrrr}2 & 5 & 10 & 5 & 20 & 5 & 20 & 40 & 90 & 90\end{array}$ $\begin{array}{rrrrrrrrrr}100 & 80 & 90 & 60 & 50 & 100 & 200 & 800 & 800 & 400\end{array}$ $\begin{array}{llllllllll}200 & 100 & 100 & 100 & 100 & 600 & 900 & 200 & 10 & 1\end{array}$

$\begin{array}{rrrrrrrrrr}5 & 10 & 20 & 20 & 10 & 5 & 10 & 30 & 40 & 50\end{array}$ $\begin{array}{rrrrrrrrrr}80 & 80 & 90 & 60 & 60 & 70 & 400 & 700 & 800 & 700\end{array}$ $\begin{array}{llllllllll}100 & 100 & 100 & 100 & 100 & 800 & 900 & 200 & 10 & 1\end{array}$ $\begin{array}{rrrrrrrrrr}5 & 5 & 20 & 20 & 5 & 10 & 10 & 40 & 30 & 30 \\ 40 & 60 & 100 & 100 & 80 & 80 & 100 & 400 & 700 & 700\end{array}$ $\begin{array}{llllllllll}100 & 100 & 100 & 100 & 200 & 800 & 800 & 400 & 10 & 1\end{array}$

$\begin{array}{llllllllll}20 & 20 & 100 & 100 & 5 & 5 & 20 & 30 & 30 & 20\end{array}$ $\begin{array}{lllllllllll}30 & 90 & 90 & 100 & 200 & 80 & 80 & 80 & 90 & 500 & 500\end{array}$ $\begin{array}{lllll}10 & 10 & 20 & 20 & 20\end{array}$

$\begin{array}{llllllllll}100 & 60 & 60 & 70 & 200 & 800 & 800 & 200 & 10 & 1\end{array}$

$\begin{array}{llllllllll}10 & 30 & 100 & 100 & 10 & 5 & 10 & 10 & 10 & 10\end{array}$ $\begin{array}{rrrrrrrrrr}90 & 90 & 100 & 300 & 100 & 80 & 70 & 80 & 100 & 300\end{array}$ $\begin{array}{llllllllll}100 & 60 & 60 & 70 & 100 & 800 & 800 & 200 & 10 & 1\end{array}$

$\begin{array}{llllllllll}20 & 30 & 200 & 200 & 20 & 10 & 10 & 10 & 10 & 10\end{array}$ $\begin{array}{rrrrrrrrrr}80 & 90 & 300 & 300 & 300 & 200 & 100 & 100 & 100 & 300\end{array}$ $\begin{array}{llllllllll}100 & 100 & 60 & 20 & 20 & 60 & 100 & 100 & 10 & 1\end{array}$ $\begin{array}{llllllllll}30 & 30 & 500 & 200 & 20 & 10 & 10 & 10 & 10 & 10\end{array}$ 
Table 2.--Listing of data for 1920-70--Continued

Card

number

909.

910.

911.

912.

913.

914.

915.

916.

917.

918.

919.

920.

921.

922.

923.

924.

925.

926.

927.

928.

929.

930.

931.
Group III: Array data--Continued

Hydraulic conductivity, in feet per second--Continued

$\begin{array}{rrrrrrrrrrrrrrrr}10 & 10 & 10 & 10 & 20 & 30 & 80 & 60 & 200 & 300 & 300 & 300 & 100 & 100 & 100 & 300 \\ 500 & 400 & 400 & 700 & 700 & 500 & 200 & 200 & 20 & 20 & 10 & 20 & 30 & 30 & 1 & 1 \\ 30 & 10 & 80 & 100 & 100 & 100 & & & & & & & & & & \\ & 10 & 20 & 20 & 20 & 20 & 30 & 80 & 700 & 700 & 40 & 10 & 5 & 5 & 5 & 5 \\ 30 & 30 & 70 & 70 & 20 & 20 & 50 & 50 & 100 & 100 & 500 & 500 & 300 & 300 & 500 & 500 \\ 800 & 400 & 400 & 400 & 700 & 700 & 50 & 50 & 10 & 10 & 10 & 20 & 30 & 30 & 5 & 10 \\ 10 & 10 & 80 & 100 & 100 & 100 & & & & & & & & & & \\ & 10 & 20 & 30 & 30 & 30 & 30 & 80 & 700 & 700 & 50 & 10 & 7 & 7 & 5 & 5 \\ 30 & 30 & 70 & 70 & 60 & 50 & 60 & 100 & 100 & 100 & 300 & 300 & 300 & 300 & 500 & 700 \\ 800 & 800 & 800 & 400 & 700 & 700 & 100 & 20 & 10 & 10 & 10 & 30 & 30 & 30 & 5 & 10 \\ 10 & 10 & 10 & 80 & 100 & 100 & & & & & & & & & & \\ & 20 & 30 & 30 & 30 & 30 & 30 & 100 & 700 & 700 & 400 & 60 & 50 & 50 & 50 & 50 \\ 100 & 100 & 200 & 200 & 200 & 200 & 100 & 100 & 200 & 300 & 300 & 300 & 600 & 600 & 800 & 800 \\ 800 & 800 & 800 & 800 & 800 & 800 & 800 & 400 & 20 & 10 & 10 & 20 & 10 & 10 & 5 & 5 \\ 20 & 20 & 10 & 80 & 100 & 100 & & & & & & & & & & \\ & 10 & 50 & 50 & 40 & 40 & 70 & 200 & 700 & 700 & 600 & 60 & 50 & 50 & 50 & 50 \\ 90 & 100 & 300 & 300 & 400 & 400 & 300 & 300 & 300 & 300 & 300 & 300 & 800 & 800 & 800 & 800 \\ 800 & 900 & 800 & 800 & 900 & 800 & 800 & 500 & 20 & 20 & 30 & 20 & 10 & 5 & 10 & 10 \\ 20 & 20 & 90 & 90 & 100 & 100 & & & & & & & & & & \end{array}$

Group III: Array data--Continued

Bottom of aquifer, in feet

(First card is parameter card)

932.

933.

934.

935.

936.

937.

938.

939.

940 .

941.

942.

943.

944.

945.

946.

947.

1

1

2

948.

949.

950.

0

1

951.

952.

$5500 \quad 5450538052905210519051305100$

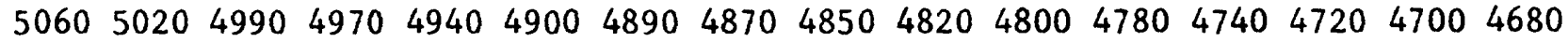

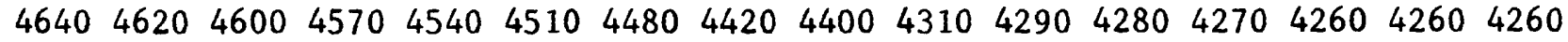
427042704280428042904300

55005440535052805210518051205090

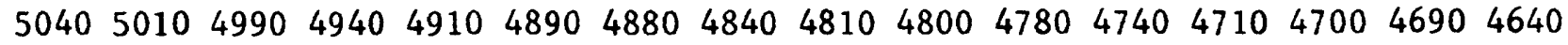
$\begin{array}{llllllllllllllllll}4610 & 4600 & 4550 & 4520 & 4500 & 4480 & 4420 & 4390 & 4320 & 4290 & 4280 & 4280 & 4270 & 4270 & 4270 & 4280\end{array}$ $4280428042904300 \quad 43104310$ 
Table 2.--Listing of data for 1920-70--Continued

Card number

953. 954. 955. 956. 957. 958. 959. 960. 961. 962. 963. 964. 965. 966. 967. 968. 969. 970. 971. 972. 973. 974. 975. 976. 977. 978. 979. 980 . 981 . 982. 983. 984. 985. 986. 987. 988 . 989. 990. 991. 992. 993. 994. 995. 996. 997. 998. 999. 1000. 1001 . 1002 .
Group III: Array data--Continued Bottom of aquifer, in feet--Continued

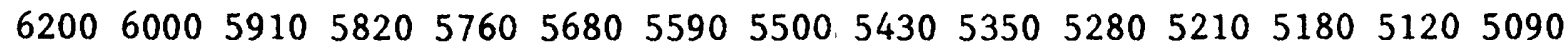
$\begin{array}{llllllllllllllllllll}5040 & 5000 & 4980 & 4940 & 4900 & 4880 & 4850 & 4820 & 4800 & 4780 & 4750 & 4720 & 4700 & 4770 & 4740 & 4710\end{array}$ $4700 \quad 4670 \quad 4730 \quad 4700 \quad 4680 \quad 4640 \quad 4600 \quad 4370 \quad 4320 \quad 4290 \quad 4290 \quad 4280 \quad 4280 \quad 4280 \quad 4280 \quad 4290$ $429043004300 \quad 431043304340$ $\begin{array}{lllllllllllllll}6210 & 6010 & 5920 & 5830 & 5770 & 5680 & 5590 & 5500 & 5430 & 5350 & 5280 & 5200 & 5170 & 5120 & 5090\end{array}$ $\begin{array}{lllllllllllllllllll}5040 & 5000 & 4970 & 4940 & 4900 & 4870 & 4840 & 4810 & 4790 & 4750 & 4730 & 4710 & 4690 & 4660 & 4630 & 4600\end{array}$ $4570 \quad 4540 \quad 4510 \quad 4490 \quad 4460 \quad 4430 \quad 4400 \quad 4360 \quad 4330 \quad 4300 \quad 4290 \quad 4280 \quad 4280 \quad 4290 \quad 4290 \quad 4300$ $\begin{array}{llllll}4310 & 4310 & 4330 & 4350 \quad 4350 \quad 4350\end{array}$

$\begin{array}{lllllllllllllll}6280 & 6080 & 5960 & 5880 & 5780 & 5690 & 5590 & 5500 & 5430 & 5350 & 5280 & 5200 & 5170 & 5120 & 5090\end{array}$ $\begin{array}{llllllllllllllllllll}5040 & 5000 & 4960 & 4930 & 4900 & 4870 & 4830 & 4800 & 4790 & 4750 & 4730 & 4700 & 4670 & 4640 & 4610 & 4590\end{array}$ $\begin{array}{lllllllllllllllllll}4560 & 4540 & 4510 & 4500 & 4460 & 4430 & 4410 & 4390 & 4360 & 4330 & 4300 & 4290 & 4290 & 4300 & 4310 & 4320\end{array}$ $\begin{array}{llllll}4350 & 4370 & 4390 & 4390 \quad 4370 \quad 4350\end{array}$

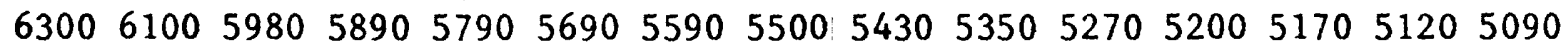

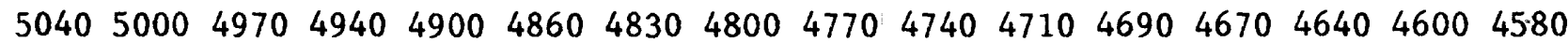
$4550 \quad 4540 \quad 4520 \quad 4500 \quad 4470 \quad 4440 \quad 4420 \quad 4400 \quad 4390 \quad 4360 \quad 4330 \quad 4310 \quad 4310 \quad 4320 \quad 4340 \quad 4360$ 439044004410441043904360

$\begin{array}{lllllllllllllll}6380 & 6180 & 6000 & 5900 & 5790 & 5690 & 5590 & 5500 & 5430 & 5350 & 5270 & 5210 & 5170 & 5120 & 5090\end{array}$ $\begin{array}{lllllllllllllllllll}5040 & 5010 & 4980 & 4940 & 4900 & 4870 & 4840 & 4800 & 4790 & 4760 & 4730 & 4700 & 4670 & 4640 & 4610 & 4590\end{array}$ $4580 \quad 4540 \quad 4520 \quad 4500 \quad 4480 \quad 4460 \quad 4440 \quad 4420 \quad 4400 \quad 4390 \quad 4370 \quad 4350 \quad 4340 \quad 4350 \quad 4380 \quad 4390$ $441044204430 \quad 4420 \quad 4400 \quad 4360$

$\begin{array}{lllllllllllllll}6400 & 620 & 6010 & 5910 & 5790 & 5700 & 5590 & 5510 & 5420 & 5350 & 5280 & 5200 & 5170 & 5120 & 5090\end{array}$ $\begin{array}{llllllllllllllllll}5050 & 5010 & 4990 & 4950 & 4900 & 4880 & 4850 & 4810 & 4790 & 4770 & 4730 & 4700 & 4690 & 4670 & 4630 & 4600\end{array}$ $4590 \quad 4570 \quad 4540 \quad 4510 \quad 4490 \quad 4480 \quad 4460 \quad 4440 \quad 4420 \quad 4410 \quad 4390 \quad 4390 \quad 4380 \quad 4390 \quad 4400 \quad 4410$ $44204440 \quad 4460 \quad 4440 \quad 44104360$ $\begin{array}{lllllllllllllll}6420 & 6250 & 6070 & 5910 & 5800 & 5700 & 5600 & 5510 & 5430 & 5350 & 5280 & 5210 & 5190 & 5140 & 5100\end{array}$ $\begin{array}{lllllllllllllllll}5170 & 5130 & 5000 & 4970 & 4920 & 4900 & 4860 & 4830 & 4800 & 4770 & 4740 & 4720 & 4700 & 4690 & 4660 & 4630\end{array}$ $\begin{array}{lllllllllllllllll}4600 & 4590 & 4570 & 4540 & 4510 & 4490 & 4480 & 4450 & 4440 & 4430 & 4410 & 4410 & 4400 & 4400 & 4410 & 4430\end{array}$ 446044904490446044104370

$\begin{array}{llllllllllllllll}64400 & 6100 & 5920 & 5810 & 5700 & 5600 & 5520 & 5440 & 5360 & 5290 & 5210 & 5200 & 5150 & 5100\end{array}$ $\begin{array}{lllllllllllllllll}5080 & 5040 & 5010 & 4980 & 4950 & 4910 & 4890 & 4850 & 4810 & 4790 & 4770 & 4750 & 4720 & 4700 & 4690 & 4660\end{array}$ $\begin{array}{llllllllllllllllll}4640 & 4620 & 4600 & 4570 & 4540 & 4510 & 4490 & 4480 & 4460 & 4440 & 4430 & 4420 & 4420 & 4440 & 4460 & 4490\end{array}$ $45004510 \quad 45104490 \quad 44204480$

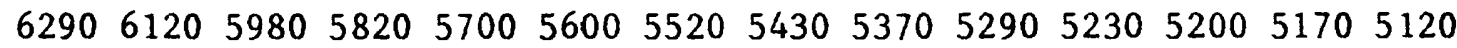
$\begin{array}{llllllllllllllllll}5100 & 5070 & 5040 & 5000 & 4970 & 4940 & 4910 & 4880 & 4850 & 4820 & 4800 & 4780 & 4750 & 4730 & 4720 & 4700\end{array}$ $\begin{array}{llllllllllllllllll}4680 & 4650 & 4620 & 4600 & 4580 & 4550 & 4510 & 4500 & 4490 & 4480 & 4480 & 4480 & 4480 & 4490 & 4500 & 4510\end{array}$ 453045504530450044304480

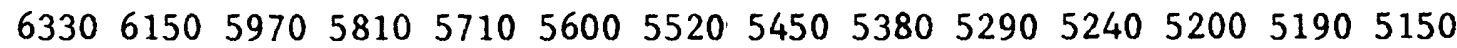
$\begin{array}{lllllllllllllllllll}5110 & 5090 & 5060 & 5030 & 5000 & 4970 & 4930 & 4900 & 4880 & 4860 & 4830 & 4800 & 4780 & 4760 & 4740 & 4720\end{array}$ $\begin{array}{lllllllllllllllllll}4700 & 4680 & 4660 & 4640 & 4610 & 4590 & 4550 & 4530 & 4520 & 4500 & 4500 & 4500 & 4500 & 4510 & 4520 & 4540\end{array}$ $4560 \quad 4580 \quad 4580 \quad 4530 \quad 4450 \quad 4390$

$\begin{array}{lllllllllllllll}6430 & 6350 & 6150 & 5980 & 5840 & 5720 & 5600 & 5520 & 5460 & 5380 & 5300 & 5260 & 5230 & 5200 & 5170\end{array}$ $\begin{array}{llllllllllllllllll}5140 & 5110 & 5080 & 5050 & 5020 & 5000 & 4970 & 4940 & 4910 & 4880 & 4860 & 4840 & 4820 & 4800 & 4780 & 4760\end{array}$ $\begin{array}{lllllllllllllllllll}4740 & 4720 & 4690 & 4660 & 4630 & 4610 & 4590 & 4570 & 4550 & 4530 & 4520 & 4520 & 4540 & 4560 & 4580 & 4600\end{array}$ $461046104610 \quad 4570 \quad 4480 \quad 4390$

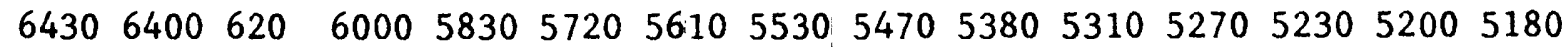
$\begin{array}{llllllllllllllllll}5160 & 5130 & 5100 & 5080 & 5050 & 5020 & 5000 & 4980 & 4950 & 4920 & 4900 & 4870 & 4850 & 4830 & 4800 & 4780\end{array}$ $\begin{array}{llllllllllllllllll}4760 & 4740 & 4720 & 4700 & 4680 & 4660 & 4640 & 4610 & 4590 & 4580 & 4580 & 4580 & 4590 & 4600 & 4630 & 4660\end{array}$ $468046804640 \quad 4590 \quad 4490 \quad 4400$

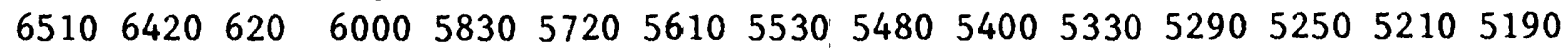
$\begin{array}{llllllllllllllll}5170 & 5140 & 5120 & 5100 & 5080 & 5050 & 5020 & 5000 & 4970 & 4940 & 4910 & 4900 & 4880 & 4860 & 4840 & 4810\end{array}$ 
Table 2.--Listing of data for 1920-70--Continued

Card

number

1003.

1004.

1005 .

1006 .

1007 .

1008 .

1009 .

1010 .

1011.

1012.

1013.

1014.

1015 .

1016.

1017.

1018 .

1019 .

1020 .

1021.

1022.

1023.

1024.

1025 .

1026 .

1027.

1028 .

1029 .

1030.

1031.

1032.

1033.

1034.

1035.

1036.

1037.

1038 .

1039.

1040 .

1041.

1042.

1043.

1044.

1045.

1046 .

1047.

1048.

1049.

1050 .

1051.

1052.
Group III: Array data--Continued

Bottom of aquifer, in feet--Continued

$4790 \quad 4770 \quad 4750 \quad 47304700 \quad 4680 \quad 4660 \quad 4640 \quad 4620 \quad 4610 \quad 4610 \quad 4610 \quad 4620 \quad 4640 \quad 4680 \quad 4700$ $47104710468046304500 \quad 4400$

$\begin{array}{lllllllllllllll}6535 & 6450 & 6220 & 6000 & 5850 & 5730 & 5620 & 5550 & 5470 & 5400 & 5340 & 5300 & 5270 & 5240 & 5210\end{array}$ $\begin{array}{lllllllllllllllll}5180 & 5160 & 5140 & 5120 & 5100 & 5070 & 5040 & 5020 & 5000 & 4980 & 4960 & 4940 & 4920 & 4900 & 4870 & 4840\end{array}$ $4820 \quad 4800 \quad 4780 \quad 4760 \quad 4740 \quad 4720 \quad 4700 \quad 4680 \quad 4670 \quad 4660 \quad 4670 \quad 4680 \quad 4690 \quad 4700 \quad 4700 \quad 4710$ $471047104700 \quad 4630 \quad 4520 \quad 4410$

$\begin{array}{lllllllllllllll}6570 & 6450 & 6220 & 6000 & 5850 & 5730 & 5620 & 5560 & 5480 & 5410 & 5360 & 5310 & 5290 & 5260 & 5230\end{array}$ $\begin{array}{llllllllllllllllll}5200 & 5180 & 5160 & 5140 & 5120 & 5100 & 5080 & 5060 & 5030 & 5010 & 4980 & 4950 & 4930 & 4920 & 4900 & 4880\end{array}$ $4860 \quad 4830 \quad 4810 \quad 4790 \quad 4770 \quad 4750 \quad 4730 \quad 4720 \quad 4710 \quad 4710 \quad 4710 \quad 4710 \quad 4720 \quad 4720 \quad 4720 \quad 4720$ $4720471047004640 \quad 45204410$

$6600 \quad 6500 \quad 6230 \quad 6010 \quad 5850 \quad 5750 \quad 5650 \quad 5570 \quad 5490 \quad 5420 \quad 5350 \quad 5320 \quad 5300 \quad 5280 \quad 5250$ $\begin{array}{lllllllllllllllll}5220 & 5200 & 5180 & 5160 & 5140 & 5120 & 5100 & 5080 & 5060 & 5040 & 5010 & 4990 & 4970 & 4950 & 4930 & 4900\end{array}$ $4880 \quad 4860 \quad 4840 \quad 4820 \quad 4800 \quad 4780 \quad 4750 \quad 4730 \quad 4730 \quad 4720 \quad 4720 \quad 4720 \quad 4720 \quad 4720 \quad 4720 \quad 4720$ $4820 \quad 471047004640 \quad 4530 \quad 4420$

$\begin{array}{lllllllllllllll}6630 & 6520 & 6250 & 6010 & 5870 & 5750 & 5650 & 5580 & 5500 & 5420 & 5380 & 5330 & 5310 & 5290 & 5260\end{array}$ $\begin{array}{llllllllllllllllll}5240 & 5210 & 5200 & 5180 & 5160 & 5140 & 5120 & 5100 & 5080 & 5060 & 5030 & 5000 & 4990 & 4970 & 4940 & 4920\end{array}$ $49004880 \quad 4860 \quad 4840 \quad 4820 \quad 4800 \quad 4780 \quad 4770 \quad 4750 \quad 4740 \quad 4730 \quad 4730 \quad 4730 \quad 4730 \quad 4730 \quad 4720$ $472047104700 \quad 4640 \quad 4540 \quad 4430$

$\begin{array}{lllllllllllllll}6650 & 6540 & 6280 & 6010 & 5870 & 5750 & 5660 & 5580 & 5500 & 5430 & 5380 & 5340 & 5320 & 5300 & 5280\end{array}$ $\begin{array}{lllllllllllllllll}5260 & 5240 & 5220 & 5200 & 5180 & 5160 & 5140 & 5120 & 5100 & 5080 & 5060 & 5030 & 5000 & 4980 & 4960 & 4940\end{array}$ $4920 \quad 4900 \quad 4880 \quad 4860 \quad 4830 \quad 4800 \quad 4790 \quad 4780 \quad 4760 \quad 4740 \quad 4730 \quad 4730 \quad 4730 \quad 4730 \quad 4730 \quad 4720$ 472047104700462045304420

$6630 \quad 6530 \quad 6280 \quad 60005870 \quad 5740 \quad 5670 \quad 5580 \quad 5510 \quad 5440 \quad 5390 \quad 5360 \quad 5330 \quad 5310 \quad 5300$ $\begin{array}{lllllllllllllllll}5280 & 5260 & 5240 & 5220 & 5190 & 5170 & 5150 & 5130 & 5110 & 5090 & 5070 & 5050 & 5030 & 5000 & 4970 & 4950\end{array}$ $4920 \quad 4900 \quad 4880 \quad 4860 \quad 4840 \quad 4820 \quad 4790 \quad 4760 \quad 4730 \quad 4730 \quad 4730 \quad 4720 \quad 4720 \quad 4720 \quad 4720 \quad 4710$ $47104710468046104530 \quad 4410$

$6600 \quad 6530 \quad 6290 \quad 6000 \quad 5860 \quad 5740 \quad 5670 \quad 5580 \quad 5510 \quad 5440 \quad 5400 \quad 5370 \quad 5340 \quad 5320 \quad 5310$ $\begin{array}{llllllllllllllllll}5290 & 5270 & 5250 & 5230 & 5210 & 5190 & 5170 & 5150 & 5130 & 5110 & 5090 & 5070 & 5040 & 5010 & 4990 & 4970\end{array}$ $494049104880486048404810 \quad 4780 \quad 4750 \quad 4720 \quad 47104700 \quad 4700 \quad 4700 \quad 4700 \quad 4700 \quad 4700$ $470046804640 \quad 4590 \quad 45204410$

$\begin{array}{llllllllllllllll}6560 & 6520 & 6300 & 6000 & 5850 & 5740 & 5670 & 5590 & 5520 & 5450 & 5410 & 5380 & 5360 & 5340 & 5320\end{array}$ $\begin{array}{llllllllllllllllll}5300 & 5280 & 5260 & 5240 & 5220 & 5200 & 5180 & 5160 & 5140 & 5120 & 5100 & 5070 & 5040 & 5010 & 4990 & 4960\end{array}$ $\begin{array}{llllllllllllllllll}4930 & 4910 & 4880 & 4860 & 4840 & 4800 & 4770 & 4730 & 4690 & 4680 & 4680 & 4670 & 4660 & 4650 & 4630 & 4610\end{array}$ $4600460045804540 \quad 4500 \quad 4430$

$\begin{array}{lllllllllllllll}6530 & 6510 & 6290 & 5990 & 5840 & 5740 & 5680 & 5600 & 5520 & 5470 & 5420 & 5390 & 5370 & 5340 & 5320\end{array}$ $\begin{array}{llllllllllllllllll}5310 & 5290 & 5270 & 5250 & 5230 & 5200 & 5180 & 5160 & 5140 & 5120 & 5100 & 5070 & 5040 & 5020 & 5000 & 4970\end{array}$ $4940 \quad 4910 \quad 4880 \quad 4860 \quad 4830 \quad 4800 \quad 4770 \quad 4730 \quad 4700 \quad 4690 \quad 4680 \quad 4660 \quad 4640 \quad 4620 \quad 4600 \quad 4590$ $45804580 \quad 4570 \quad 4560 \quad 4480 \quad 4450$

$\begin{array}{lllllllllllllll}6490 & 6290 & 5980 & 5820 & 5740 & 5680 & 5600 & 5520 & 5470 & 5430 & 5390 & 5380 & 5360 & 5340\end{array}$ $\begin{array}{lllllllllllllllll}5320 & 5300 & 5280 & 5260 & 5240 & 5220 & 5190 & 5170 & 5150 & 5130 & 5100 & 5080 & 5050 & 5020 & 5000 & 4970\end{array}$ $\begin{array}{lllllllllllllllllll}49 & 4910 & 4880 & 4850 & 4830 & 4810 & 4790 & 4760 & 4730 & 4710 & 4700 & 4680 & 4660 & 4630 & 4600 & 4600\end{array}$ $4590456045304500 \quad 44704450$

$\begin{array}{lllllllllllllll}6490 & 6290 & 5970 & 5810 & 5750 & 5690 & 5600 & 5520 & 5470 & 5430 & 5400 & 5380 & 5360 & 5340\end{array}$ $\begin{array}{lllllllllllllllll}5330 & 5310 & 5290 & 5270 & 5250 & 5220 & 5200 & 5180 & 5150 & 5120 & 5100 & 5080 & 5050 & 5020 & 5000 & 4970\end{array}$ $4940 \quad 4910 \quad 4890 \quad 4870 \quad 4850 \quad 4830 \quad 4810 \quad 4790 \quad 4770 \quad 4740 \quad 4720 \quad 4700 \quad 4690 \quad 4670 \quad 4640 \quad 4620$ 460045904560450044804450

$\begin{array}{lllllllllllll}6280 & 5920 & 5800 & 5740 & 5700 & 5610 & 5520 & 5480 & 5440 & 5410 & 5390 & 5370 & 5350\end{array}$ $\begin{array}{llllllllllllllllll}5330 & 5320 & 5300 & 5280 & 5250 & 5220 & 5200 & 5170 & 5140 & 5120 & 5100 & 5080 & 5050 & 5020 & 5000 & 4970\end{array}$ $4940 \quad 49204900 \quad 4880 \quad 4860 \quad 4840 \quad 4820 \quad 4800 \quad 4790 \quad 4770 \quad 4750 \quad 4730 \quad 4710 \quad 4690 \quad 4680 \quad 4660$ 464046204600455044904470 
Table 2.--Listing of data for 1920-70--Continued

Card

number

1053.

1054.

1055.

1056 .

1057 .

1058.

1059 .

1060 .

1061.

1062 .

1063.

1064.

1065.

1066.

1067.

1068 .

1069.

1070.

1071 .

1072 .

1073.

1074.

1075 .

1076.

1077.

1078 .

1079 .

1080 .

1081 .

1082.

1083.

1084 .

1085 .

1086.

1087.

1088 .

1089.

1090.

1091.

1092.

1093.

1094.

1095.

1096.

1097.

1098.

1099.

1100 .

1101.

1102 .
Group III: Array data--Continued

Bottom of aquifer, in feet--Continued

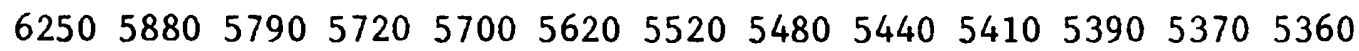

$\begin{array}{llllllllllllllll}5340 & 5320 & 5310 & 5290 & 5270 & 5240 & 5200 & 5180 & 5150 & 5120 & 5100 & 5080 & 5060 & 5030 & 5000 & 4970\end{array}$ $\begin{array}{lllllll}4940 & 4920 & 4900 & 4880 & 4860 \quad 4840 \quad 4830 & 4820 \quad 4810 \quad 4800 \quad 4780 \quad 4760 \quad 4740 \quad 4720 \quad 4700 \quad 4690\end{array}$ 468046604630459045104480

$\begin{array}{lllllllllllll}6180 & 5880 & 5770 & 5700 & 5700 & 5620 & 5520 & 5480 & 5440 & 5420 & 5400 & 5380 & 5370\end{array}$ $\begin{array}{lllllllllllllllll}5350 & 5330 & 5310 & 5300 & 5270 & 5240 & 5210 & 5180 & 5150 & 5120 & 5100 & 5070 & 5040 & 5020 & 5000 & 4980\end{array}$ $\begin{array}{llllllllllllllllllll}4960 & 4940 & 4920 & 4900 & 4880 & 4860 & 4840 & 4830 & 4820 & 4810 & 4800 & 4780 & 4760 & 4740 & 4720 & 4710\end{array}$ 470046804650461045604490

$\begin{array}{lllllllllllll}6150 & 5850 & 5740 & 5700 & 5700 & 5620 & 5530 & 5480 & 5440 & 5430 & 5410 & 5390 & 5380\end{array}$

$\begin{array}{llllllllllllllllll}5360 & 5340 & 5320 & 5300 & 5280 & 5260 & 5220 & 5190 & 5150 & 5110 & 5090 & 5070 & 5040 & 5020 & 5000 & 4980\end{array}$

$\begin{array}{lllllllllllllllllll}4960 & 4940 & 4920 & 4900 & 4890 & 4880 & 4860 & 4840 & 4830 & 4820 & 4810 & 4800 & 4790 & 4770 & 4750 & 4730\end{array}$

$4710469046704640 \quad 45904510$

$\begin{array}{llllllllllllll}6500 & 6100 & 5830 & 5730 & 5700 & 5700 & 5630 & 5520 & 5480 & 5450 & 5430 & 5410 & 5390 & 5370\end{array}$

$\begin{array}{llllllllllllllllll}5350 & 5340 & 5320 & 5300 & 5280 & 5250 & 5220 & 5190 & 5160 & 5120 & 5090 & 5060 & 5040 & 5020 & 4990 & 4970\end{array}$

$\begin{array}{lllllllllllllllllll}4950 & 4940 & 4930 & 4920 & 4900 & 4880 & 4870 & 4860 & 4850 & 4840 & 4830 & 4820 & 4800 & 4780 & 4760 & 4740\end{array}$

472047004680465046004540

$\begin{array}{llllllllllllllll}6480 & 6100 & 5820 & 5700 & 5690 & 5700 & 5660 & 5550 & 5480 & 5450 & 5430 & 5420 & 5400 & 5380\end{array}$

$\begin{array}{lllllllllllllllll}5360 & 5340 & 5330 & 5310 & 5290 & 5270 & 5240 & 5200 & 5160 & 5120 & 5090 & 5070 & 5040 & 5020 & 4990 & 4980\end{array}$

$\begin{array}{lllllllllllllllllll}4960 & 4950 & 4940 & 4930 & 4920 & 4900 & 4890 & 4880 & 4870 & 4850 & 4840 & 4830 & 4810 & 4800 & 4790 & 4770\end{array}$

$475047304700 \quad 46704630 \quad 4580$

$\begin{array}{llllllllllllllll}6450 & 6080 & 5810 & 5700 & 5680 & 5700 & 5680 & 5580 & 5490 & 5460 & 5440 & 5420 & 5400 & 5390\end{array}$

$\begin{array}{lllllllllllllllll}5370 & 5350 & 5330 & 5320 & 5300 & 5270 & 5240 & 5200 & 5170 & 5140 & 5100 & 5070 & 5040 & 5010 & 4990 & 4980\end{array}$

$\begin{array}{llllllllllllllllll}4970 & 4960 & 4950 & 4940 & 4930 & 4920 & 4910 & 4900 & 4880 & 4870 & 4850 & 4840 & 4830 & 4820 & 4800 & 4790\end{array}$

477047504720468046404600

$\begin{array}{llllllllllllll}6450 & 6080 & 5810 & 5700 & 5680 & 5700 & 5690 & 5590 & 5490 & 5470 & 5450 & 5430 & 5410 & 5390\end{array}$

$\begin{array}{llllllllllllllllll}5380 & 5370 & 5350 & 5330 & 5300 & 5270 & 5240 & 5200 & 5170 & 5140 & 5100 & 5080 & 5060 & 5030 & 5000 & 4990\end{array}$

$\begin{array}{llllllllllllllllll}4980 & 4970 & 4960 & 4950 & 4940 & 4930 & 4920 & 4910 & 4890 & 4880 & 4870 & 4860 & 4840 & 4830 & 4820 & 4800\end{array}$

$47904770 \quad 4740 \quad 4700 \quad 46604610$

$\begin{array}{llllllllllllllll}6900 & 6400 & 6080 & 5820 & 5700 & 5680 & 5690 & 5700 & 5600 & 5500 & 5470 & 5450 & 5440 & 5420 & 5400\end{array}$

$\begin{array}{lllllllllllllllll}5380 & 5370 & 5350 & 5330 & 5300 & 5280 & 5240 & 5200 & 5180 & 5140 & 5100 & 5090 & 5070 & 5040 & 5020 & 5000\end{array}$

$4990 \quad 4980 \quad 4970 \quad 4960 \quad 4950 \quad 4940 \quad 4930 \quad 4920 \quad 4900 \quad 4890 \quad 4880 \quad 4870 \quad 4850 \quad 4830 \quad 4820 \quad 4810$

$48004780 \quad 4740 \quad 471046804620$

$\begin{array}{llllllllllllllll}6900 & 6420 & 6090 & 5880 & 5750 & 5690 & 5690 & 5700 & 5630 & 5510 & 5480 & 5450 & 5440 & 5420 & 5400\end{array}$

$\begin{array}{lllllllllllllllll}5390 & 5380 & 5360 & 5330 & 5310 & 5290 & 5250 & 5210 & 5180 & 5150 & 5120 & 5100 & 5080 & 5050 & 5030 & 5010\end{array}$

$\begin{array}{llllllllllllllllll}4990 & 4980 & 4970 & 4960 & 4950 & 4940 & 4930 & 4920 & 4910 & 4900 & 4890 & 4880 & 4860 & 4840 & 4830 & 4820\end{array}$

480047804750472046804630

$\begin{array}{lllllllllllllll}6900 & 6420 & 6090 & 5880 & 5720 & 5690 & 5690 & 5700 & 5630 & 5520 & 5480 & 5460 & 5440 & 5430 & 5410\end{array}$

$\begin{array}{llllllllllllllll}5390 & 5380 & 5350 & 5330 & 5310 & 5290 & 5270 & 5240 & 5190 & 5170 & 5140 & 5100 & 5090 & 5070 & 5050 & 5030\end{array}$

$\begin{array}{lllllllllllllllll}5010 & 5000 & 4990 & 4980 & 4970 & 4960 & 4940 & 4930 & 4920 & 4910 & 4890 & 4880 & 4870 & 4850 & 4840 & 4820\end{array}$

$4800 \quad 4780 \quad 4760 \quad 4720 \quad 4680 \quad 4630$

$\begin{array}{llllllllllllllll}6850 & 6420 & 6110 & 5900 & 5780 & 5700 & 5690 & 5700 & 5660 & 5560 & 5490 & 5470 & 5450 & 5430 & 5410\end{array}$

$\begin{array}{lllllllllllllllll}5390 & 5380 & 5360 & 5340 & 5310 & 5280 & 5250 & 5220 & 5190 & 5160 & 5130 & 5110 & 5100 & 5080 & 5060 & 5040\end{array}$

$\begin{array}{lllllllllllllllll}5030 & 5020 & 5000 & 4990 & 4980 & 4970 & 4960 & 4940 & 4930 & 4920 & 4900 & 4880 & 4870 & 4860 & 4840 & 4820\end{array}$

$4800 \quad 4780 \quad 4750 \quad 47204680 \quad 4640$

$\begin{array}{llllllllllllllll}6850 & 6400 & 6120 & 6040 & 5820 & 5720 & 5690 & 5700 & 5690 & 5590 & 5500 & 5470 & 5460 & 5440 & 5420\end{array}$

$\begin{array}{lllllllllllllllll}5400 & 5380 & 5360 & 5340 & 5320 & 5290 & 5260 & 5230 & 5200 & 5180 & 5160 & 5130 & 5110 & 5090 & 5070 & 5050\end{array}$

$\begin{array}{llllllllllllllllll}5040 & 5030 & 5020 & 5000 & 4990 & 4980 & 4970 & 4960 & 4940 & 4920 & 4900 & 4890 & 4870 & 4850 & 4830 & 4820\end{array}$

$4800478047604730 \quad 4680 \quad 4640$

$\begin{array}{lllllllllllllllll}6850 & 6400 & 6150 & 5980 & 5880 & 5780 & 5700 & 5700 & 5700 & 5610 & 5520 & 5480 & 5460 & 5440 & 5420\end{array}$

$\begin{array}{llllllllllllllll}5400 & 5390 & 5370 & 5350 & 5320 & 5290 & 5260 & 5230 & 5200 & 5180 & 5160 & 5140 & 5120 & 5100 & 5090 & 5070\end{array}$ 
Table 2.--Listing of data for 1920-70--Continued

Card

number

1103.

1104.

1105 .

1106.

1107.

1108 .

1109 .

1110 .

1111 .

1112 .

1113.

1114.

1115 .

1116.

1117.

1118 .

1119 .

1120 .

1121 .

1122 .

1123.

1124.

1125 .

1126 .

1127.

1128 .

1129 .

1130 .

1131.

1132 .

1133.

1134.

1135 .

1136.

1137.

1138 .

1139 .

1140 .
Group III: Array data--Continued

Bottom of aquifer, in feet--Continued

$5050 \quad 5040502050105000 \quad 4980 \quad 4960 \quad 4940 \quad 4930 \quad 4920 \quad 4910 \quad 4890 \quad 4880 \quad 4860 \quad 4840 \quad 4820$

$4800 \quad 4780 \quad 4760 \quad 4720 \quad 4680 \quad 4640$

$\begin{array}{lllllllllllllll}6800 & 6420 & 6170 & 6010 & 5910 & 5810 & 5750 & 5730 & 5720 & 5640 & 5550 & 5500 & 5490 & 5460 & 5430\end{array}$

$\begin{array}{llllllllllllllllll}5410 & 5390 & 5370 & 5350 & 5320 & 5300 & 5270 & 5240 & 5210 & 5190 & 5170 & 5150 & 5130 & 5110 & 5090 & 5080\end{array}$

$5060 \quad 5050 \quad 5030 \quad 5020 \quad 5000 \quad 4990 \quad 4970 \quad 4950 \quad 4940 \quad 4930 \quad 4910 \quad 4890 \quad 4880 \quad 4860 \quad 4830 \quad 4810$

$4790 \quad 4770 \quad 4740 \quad 471046804640$

$\begin{array}{llllllllllllllll}6770 & 6440 & 6180 & 6050 & 5930 & 5860 & 5790 & 5770 & 5730 & 5630 & 5550 & 5500 & 5490 & 5460 & 5440\end{array}$

$\begin{array}{llllllllllllllllll}5410 & 5390 & 5370 & 5350 & 5330 & 5300 & 5280 & 5260 & 5230 & 5200 & 5180 & 5160 & 5140 & 5120 & 5100 & 5080\end{array}$

$\begin{array}{lllllllllllllllll}5070 & 5060 & 5040 & 5030 & 5010 & 4990 & 4970 & 4960 & 4940 & 4930 & 4910 & 4890 & 4870 & 4850 & 4830 & 4810\end{array}$

$4790 \quad 4780 \quad 4740 \quad 4710 \quad 46804640$

$\begin{array}{lllllllllllllll}6680 & 6450 & 6200 & 6050 & 5930 & 5860 & 5780 & 5720 & 5750 & 5700 & 5600 & 5540 & 5500 & 5490 & 5470\end{array}$

$\begin{array}{llllllllllllllllll}5440 & 5400 & 5380 & 5360 & 5340 & 5310 & 5280 & 5250 & 5230 & 5200 & 5180 & 5170 & 5150 & 5130 & 5110 & 5090\end{array}$

$5070 \quad 5060 \quad 5040 \quad 5030 \quad 5010 \quad 4990 \quad 4980 \quad 4970 \quad 4950 \quad 4930 \quad 4910 \quad 4890 \quad 4880 \quad 4860 \quad 4840 \quad 4810$

479047704730470046804640

$\begin{array}{lllllllllllllll}6600 & 6420 & 6220 & 6050 & 5930 & 5830 & 5750 & 5650 & 5700 & 5710 & 5610 & 5570 & 5530 & 5500 & 5480\end{array}$

$\begin{array}{llllllllllllllll}5450 & 5410 & 5390 & 5370 & 5340 & 5310 & 5290 & 5270 & 5240 & 5210 & 5190 & 5170 & 5150 & 5130 & 5120 & 5100\end{array}$

$5080 \quad 50605040 \quad 50305010 \quad 4990 \quad 4980 \quad 4960 \quad 4940 \quad 4930 \quad 4910 \quad 4890 \quad 4870 \quad 4850 \quad 4830 \quad 4800$

$47804760 \quad 4730 \quad 4700 \quad 46704630$

$\begin{array}{lllllllllllllll}6530 & 6350 & 6180 & 6000 & 5850 & 5750 & 5700 & 5630 & 5640 & 5660 & 5680 & 5590 & 5550 & 5510 & 5490\end{array}$

$\begin{array}{llllllllllllllllll}5440 & 5410 & 5390 & 5370 & 5340 & 5300 & 5290 & 5250 & 5230 & 5200 & 5190 & 5140 & 5150 & 5130 & 5120 & 5100\end{array}$

$5080506050405030 \quad 5010 \quad 4990 \quad 4980 \quad 4970 \quad 4950 \quad 4930 \quad 4910 \quad 4890 \quad 4870 \quad 4850 \quad 4830 \quad 4800$

478047604730470046704630

$\begin{array}{lllllllllllllll}6450 & 6280 & 6120 & 5970 & 5840 & 5740 & 5650 & 5610 & 5590 & 5590 & 5620 & 5610 & 5580 & 5530 & 5500\end{array}$

$\begin{array}{lllllllllllllllll}5430 & 5390 & 5340 & 5320 & 5300 & 5280 & 5270 & 5250 & 5220 & 5190 & 5180 & 5140 & 5140 & 5120 & 5100 & 5080\end{array}$

$50805070 \quad 5050 \quad 5030 \quad 5010 \quad 4990 \quad 4980 \quad 4970 \quad 4950 \quad 4930 \quad 4910 \quad 4890 \quad 4870 \quad 4840 \quad 4810 \quad 4790$

$47704740 \quad 4720469046604630$

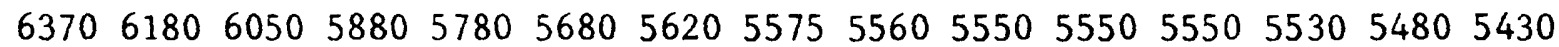

$\begin{array}{lllllllllllllllll}5380 & 5330 & 5310 & 5280 & 5270 & 5240 & 5220 & 5200 & 5190 & 5160 & 5150 & 5130 & 5110 & 5090 & 5070 & 5060\end{array}$

$5040 \quad 5040 \quad 5020 \quad 5010 \quad 5010 \quad 4990 \quad 4980 \quad 4960 \quad 4940 \quad 4920 \quad 4900 \quad 4890 \quad 4860 \quad 4830 \quad 4800 \quad 4780$

$47604740 \quad 4720468046504620$

$\begin{array}{lllllllllllllll}6250 & 6100 & 5930 & 5800 & 5720 & 5650 & 5590 & 5550 & 5530 & 5510 & 5490 & 5470 & 5450 & 5410 & 5360\end{array}$

$\begin{array}{llllllllllllllll}5330 & 5300 & 5270 & 5250 & 5220 & 5200 & 5190 & 5170 & 5160 & 5130 & 5120 & 5100 & 5080 & 5070 & 5040 & 5030\end{array}$

$5010 \quad 5000 \quad 4990 \quad 4980 \quad 4980 \quad 4970 \quad 4970 \quad 4960 \quad 4940 \quad 4920 \quad 4900 \quad 4870 \quad 4840 \quad 4810 \quad 4790 \quad 4770$

$47504730 \quad 4700 \quad 4670 \quad 4640 \quad 4610$

0

0

0

0

Group III: Array data--Continued

Specific yield, dimensionless

(Parameter card)

1141.

.15 
Table 2.--Listing of data for 1920-70--Continued

Card

1142.

1143.

1144.

1145 .

1146 .

1147 .

1148 .

1149 .

1150 .

1151.

1152 .

1153.

1154 .

1155 .

1156 .

1157.

1158 .

1159 .

1160 .

1161.

1162.

1163.

1164.

1165.

1166 .

1167.

1168 .

1169 .

1170 .

1171 .

1172 .

1173.

1174.

1175 .

1176.

1177.

1178 .

1179 .

1180 .

1181 .

1182 .

1183.

1184.

1185 .

1186 .

1187.

1188 .

1189 .

1190 .

1191.
Group III: Array data--Continued

Confining bed thickness at stream nodes, in feet

(First card is parameter card)
4

5

5

5

5

5

5

6

6

6

6

6

6

6

7

7

7

7

7

7

7

7

7

8

8

9

9

9

9

9

9

9

10

10

10

10

11

11

11

11

11

11

11

11

11

12

13

13

13 $\begin{array}{rl}2 & 100 \\ 1.0 & \\ 1.0 & \\ 1.0 & \end{array}$

1.0

1.0

1.0

1.0

1.0

1.0

1.0

1.0

1.0

1.0

1.0

1.0

1.0

1.0

1.0

1.0

1.0

1.0

1.0

1.0

1.0

1.0

1.0

1.0

1.0

1.0

1.0

1.0

1.0

1.0

1.0

1.0

1.0

1.0

1.0

1.0

1.0

1.0

1.0

1.0

1.0

1.0

1.0

1.0

1.0

1.0

1.0 
Table 2.--Listing of data for 1920-70--Continued

Card number

1192. 1193. 1194. 1195. 1196. 1197. 1198. 1199. 1200 .

1201 .

1202.

1203.

1204.

1205 .

1206.

1207.

1208 .

1209 .

1210 .

1211.

1212 .

1213.

1214.

1215 .

1216 .

1217.

1218 .

1219 .

1220 .

1221 .

1222 .

1223.

1224.

1225 .

1226 .

1227.

1228.

1229 .

1230 .

1231.

1232 .

1233.

1234 .

1235 .

1236.

1237.

1238.

1239.

1241 .

1242.
Group III: Array data--Continued

Confining bed thickness at stream nodes, in feet--Continued

14

14

28

29

29

30

30

30

31

31

31

31

32

32

33

34

34

35

35

35

35

35

35

35

35

35

35

35

35

36

36

36

36

36

36

37

37

38

39

40

41

42

43

44

45

46

47

48

50

51 $\begin{array}{rr}3 & 1.0 \\ 4 & 1.0 \\ 54 & 1.0 \\ 52 & 1.0 \\ 53 & 1.0 \\ 49 & 1.0\end{array}$

$50 \quad 1.0$

$51 \quad 1.0$

1.0

1.0

1.0

1.0

1.0

1.0

1.0

1.0

1.0

1.0

1.0

1.0

1.0

1.0

1.0

1.0

1.0

1.0

1.0

1.0

1.0

1.0

1.0

1.0

1.0

1.0

1.0

1.0

1.0

1.0

1.0

1.0

1.0

1.0

1.0

1.0

1.0

1.0

1.0

1.0

1.0

1.0 
Table 2.--Iisting of data for 1920-70--Continued

Card

number

1243.

1244 .

1245 .

1246 .

1247.

1248 .

1249 .

1250 .

1251 .

1252 .

1253.

1254.

1255.

1256.

1257 .

1258 .

1259 .

1260 .

1261 .

1262 .

1263.

1264 .

1265 .

1266 .

1267.

1268 .

1269 .

1270 .

1271 .

1272 .

1273.

1274 .

1275 .

1276 .

1277.

1278 .

1279 .

1280 .

1281 .

1282 .

1283 .

1284.

1285 .

1286 .

1287.

1288 .

1289.

1290 .

1291.

1292.
Group III: Array data--Continued

Initial rate of gain in stream cells, in cubic feet per second (First card is parameter card)

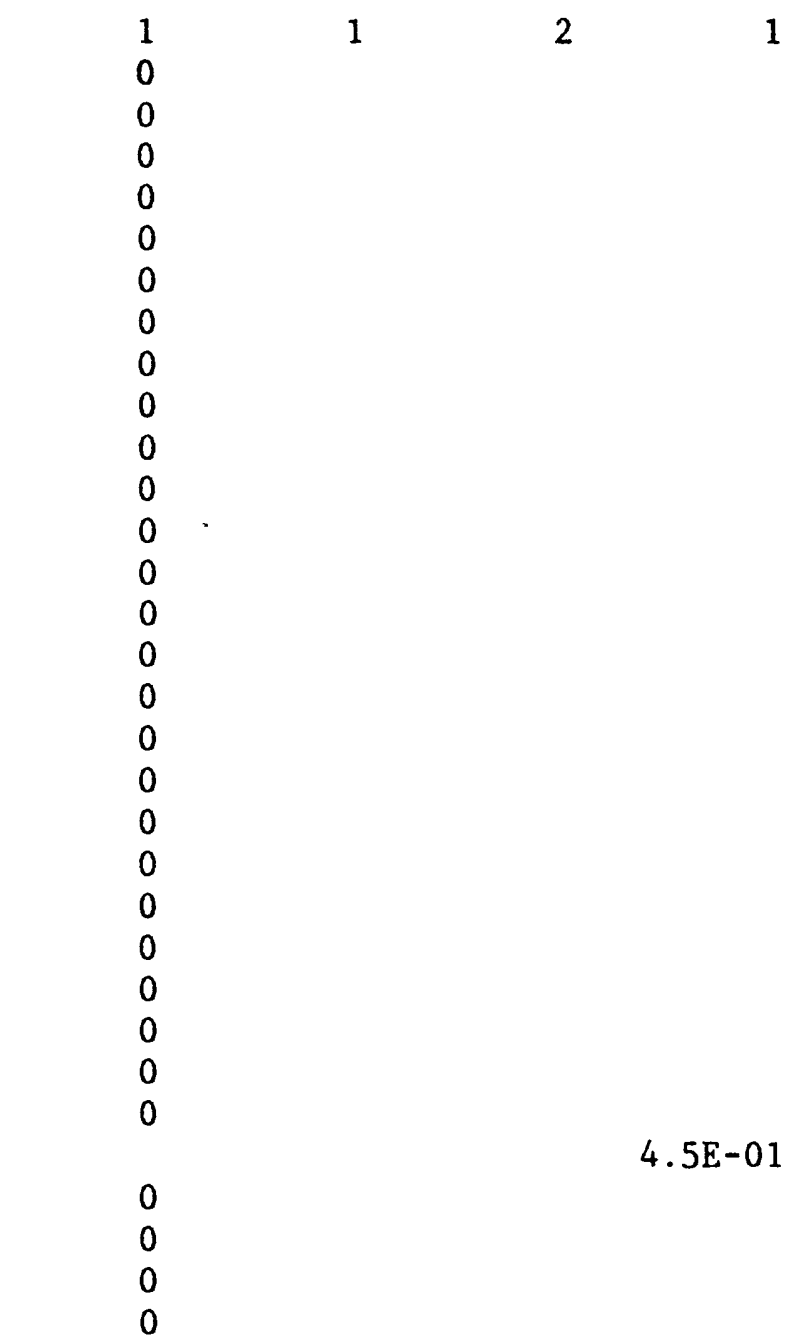

$9.0 \mathrm{E}-02 \quad 9.0 \mathrm{E}-02$

$1.0 \mathrm{E}-01 \quad 1.0 \mathrm{E}-01 \quad 1.0 \mathrm{E}-01$
$0.5 \quad 3.0-07$

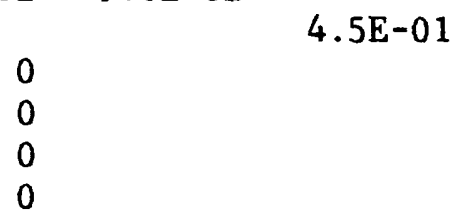

$4.5 \mathrm{E}-01$

1. $8 \mathrm{E}-01$

3. $0 \mathrm{E}-01 \quad 1.0 \mathrm{E}-01$

$3 . \mathrm{OE}-01$
0
0
0
0

3. $0 \mathrm{E}-01$

3. $0 \mathrm{E}-01$

$\begin{array}{rll}1.1 \mathrm{E}-01 & 5.0 \mathrm{E}-01 & 3.0 \mathrm{E}-01 \\ 1.2 \mathrm{E}-01 & 1.5 \mathrm{E}-01\end{array}$

$1.7 \mathrm{E}-01 \quad 3.0 \mathrm{E}-01 \quad 3.0 \mathrm{E}-01 \quad 3.0 \mathrm{E}-01$ 
Card Group III: Array data--Continued

number Initial rate of gain in stream cells, in cubic feet per second--Continued

1293.

1294.

1295.

1296.

1297.

1298.

1299.

1300 .

1301.

1302 .

1303.

1304.

1305.

1306.

1307.

1308 .

1309 .

1310 .

1311.

1312 .

1313.

1314.

1315.

1316.

1317.

1318.

1319.

1320 .

1321.

1322 .

1323.

1324.

1325 .

1326 .

1327.

1328.

1329 .

1330 .

1331.

1332.

1333.

1334.

1335.

1336.

1337.

1338.

1339.

1340 .

1341.

1342.

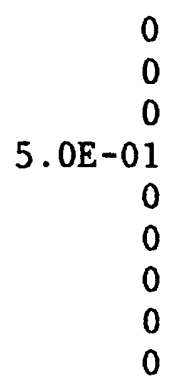

$6.0 \mathrm{E}-01$

5.5E-01

5. $5 \mathrm{E}-01$

5. $0 \mathrm{E}-01$

$6.9 \mathrm{E}-01 \quad 4.8 \mathrm{E}-01$

7. $0 \mathrm{E}-01$

$6.5 \mathrm{E}-01$

$6.5 \mathrm{E}-01$

$6.0 \mathrm{E}-01$

0

0

0

4. $5 \mathrm{E}-01$

$5.5 E-01$

$6.7 E-01$

9. $0 \mathrm{E}-01$

7. $5 \mathrm{E}-01$

7. $5 E-01$

7. $0 \mathrm{E}-01$

$7.0 \mathrm{E}-01$

$1.53 \mathrm{E} 00$

0

0

0

0

0

0

0

0

0

0

0

0

0

0

0

0

0

0

0

0

0

0
$4.9 E-01$

$5.0 \mathrm{E}-01$

$3.5 E-01$

$$
\text { 9. } 0 \mathrm{E}-02 \quad 9.0 \mathrm{E}-02
$$


Table 2.--Listing of data for 1920-70--Continued

Card

Group III: Array data--Continued

number Initial rate of gain in stream cells, in cubic feet per second--Continued

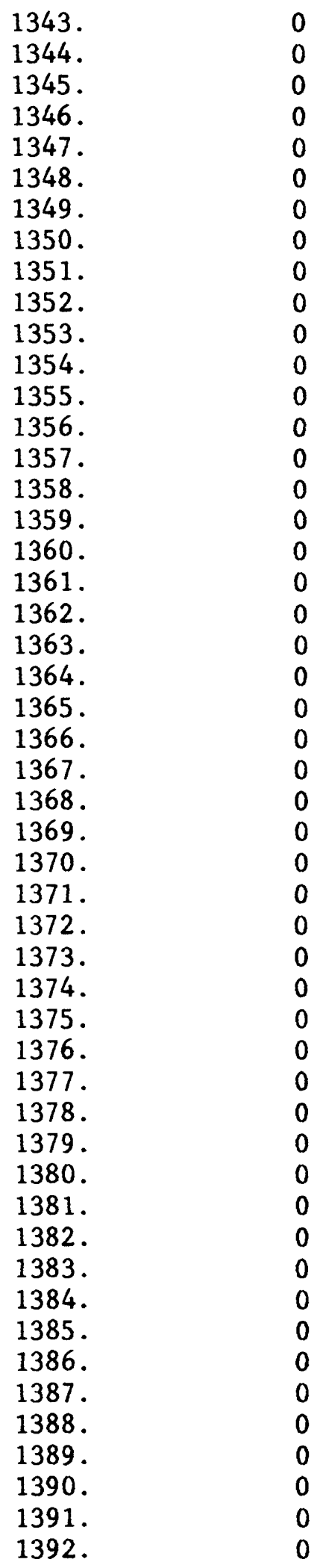


Table 2.--Listing of data for 1920-70--Continued

Card

Group III: Array data--Continued

number Initial rate of gain in stream cells, in cubic feet per second--Continued 1393.

1394. 0

1395. 0

1396.

1397.

1398.

1399.

1400 . 0

1401 . 0

1402 . 0

$1403 . \quad 0$

1404 . 0

1405 . 0

$1406 . \quad 0$

1407 . 0

1408 . 0

1409 . 0

1410 . 0

1411 . 0

1412 . 0

$1413 . \quad 0$

1414 . 0

1415 . 0

1416 . 0

$1417 . \quad 0$

1418 . 0

1419 . 0

1420 . 0

1421 . 0

1422 . 0

1423 . 0

1424 . 0

1425 . 0

1426 . 0

1427.0

1428 . 0

$1429 . \quad 0$

1430 . 0

$1431 . \quad 0$

1432. 0

$1433 . \quad 0$

$1434 . \quad 0$

1435 . 0

1436.0

$1437 . \quad 0$

1438 . 0

1439 .

1441 . 0

$3.0 \mathrm{E} 00$

1442 . 0 
Table 2.--Listing of data for 1920-70--Continued

Card Group III: Array data--Continued

number

1443.

1444.

1445 .

1446 .

1447 .

1448 .

1449 .

1450 .

1451 .

1452 .

1453.

1454 .

1455 .

1456.

1457 .

1458 .

1459 .

1460 .

1461.

1462.

1463.

1464 .

1465 .

1466 .

1467.

1468 .

1469 .

1470 .

1471 .

1472 .

1473.

1474 .

1475 .

1476 .

1477.

1478 .

1479 .

1480 .

1481 .

1482 .

1483 .

1484 .

1485 .

1486 .

1487 .

1488 .

1489 .

1490 .

1491 .

1492.

2. $0 \mathrm{E} \quad 00 \quad 3.0 \mathrm{E} \quad 00$

$3.9 E-01$

2. $0 \mathrm{E}-02$

0

0

0

0

0
$6.0 \mathrm{E}-02$

$6.0 \mathrm{E}-02$

0

0

0

0

0

1. $0 \mathrm{E}-01$

0

0

0

0

0

0

$\begin{array}{rrrrrrrrrrrr}1.5 \mathrm{E}-01 & & & -2.0 \mathrm{E}-01 & -1.5 \mathrm{E} & 00 & -1.5 \mathrm{E} & 00 & -1.5 \mathrm{E} & 00 \\ -1.5 \mathrm{E} & 00 & -1.5 \mathrm{E} & 00 & -5.0 \mathrm{E}-01 & -2.0 \mathrm{E}-01 & -1.0 \mathrm{E}-01 & -1.0 \mathrm{E}-01 & & & \end{array}$ 0

0

0

0

0

0

$-1.0 \mathrm{E}-01-1.0 \mathrm{E}-01-1.0 \mathrm{E}-01-1.0 \mathrm{E}-01$ 
Table 2.--Listing of data for 1920-70--Continued

Card Group III: Array data--Continued

number Initial rate of gain in stream cells, in cubic feet per second--Continued

1493

1494.

1495.

1496.

1497.

1498.

1499 .

1500 .

1501 .

1502 .

1503.

1504 .

1505 .

1506.

1507 .

1508 .

1509 .

1510.

1511.

1512 .

1513.

1514 .

1515 .

1516 .

1517.

1518.

1519 .

1520 .

1521.

1522 .

1523.

1524 .

1525 .

1526 .

1527.

1528 .

1529 .

1530 .

1531.

1532.

1533.

1534.

1535.

1536.

1537.

1538 .

1539.

1540 .

1541 .

1542 .
0

0

0

0

0

0

0

0

0

0

0

0

0

0

0

0

0

0

0

0

0

0

0

0

0

0

0

0

0

0

0

0

0

0

0

0

0

0

0

0

0

0

0

0

0

0

5. OE-02
$-5.0 \mathrm{E}-01 \quad-5.0 \mathrm{E}-01$

5. $\mathrm{OE}-01$ 
Table 2.--Listing of data for 1920-70--Continued

Card number

1543.

1544 .

1545 .

1546 .

1547.

1548 .

1549 .

1550 .

1551 .

1552 .

1553.

1554 .

1555 .

1556.

1557.

1558.

1559 .

1560 .

1561.

1562.

1563.

1564.

1565.

1566 .

1567.

1568 .

1569 .

1570 .

1571 .

1572 .

1573.

1574 .

1575 .

1576 .

1577.

1578 .

1579 .

1580 .

1581 .

1582 .

1583.

1584 .

1585 .

1586 .

1587.

1588 .

1589 .

1590 .

1591.

1592.
Group III: Array data--Continued

Initial rate of gain in stream cells, in cubic feet per second--Continued
0

0

0

0

0

0

1. $0 \mathrm{E}-01$

\section{0}

0

0

0

0

0

$-1.0 \mathrm{E}-01$

0

0

0

0

0

0

0

0

0

0

0

0

0

0

0

0

0

0

0

0

0

0

0

0

0

0

0

0

0 
Table 2.--Listing of data for 1920-70--Continued

Card Group III: Array data--Continued

number Initial rate of gain in stream cells, in cubic feet per second--Continued 1593.

1594. 0

1595.

1596.

1597.

1598 . 0

1599 . 0

1600 . 0

1601 . 0

1602. 0

1603.

1604 . 0

1605 . 0

1606 . 0

1607.

Group III: Array data--Continued

Head at stream nodes, in feet

(Parameter card)

1608 .

0

$0 \quad 2$

Group III: Array data--Continued Top of aquifer, altitude of stream, in feet

(Parameter card)

1609 .

0

2

Group III: Array data--Continued

Recharge rate, in feet per second (Parameter card)

1610.

2. 19E-09

Number of streams and node identification

1611. 5

1612. $10 \quad 15 \quad 20 \quad 30 \quad 35$

Test card to calculate initial recharge/discharge at constant-flux boundary nodes

1613. 
Table 2.--Listing of data for 1920-70--Continued

Card number

Group IV: Data that change with pumping period

1614.

1

$0 \quad 145$

$18615 \quad 1000$

1.5

24

0

Parameters for streamflow accounting procedure

Number of streams

1615 . 5

\begin{tabular}{|c|c|c|c|c|c|}
\hline \multirow{2}{*}{$\begin{array}{c}\text { Stream } \\
\text { identification }\end{array}$} & \multirow{2}{*}{ Upstream } & \multirow{2}{*}{$\frac{\text { node }}{\mathrm{J}}$} & \multirow{2}{*}{$\begin{array}{l}\text { Inflow } \\
\text { rate, in } \\
\text { cubic feet } \\
\text { per second }\end{array}$} & \multicolumn{2}{|c|}{$\begin{array}{c}\text { Last } \\
\text { downstream } \\
\text { node } \\
\end{array}$} \\
\hline & & & & $\mathrm{I}$ & $\mathrm{J}$ \\
\hline 10 & 14 & 3 & .09 & 4 & 44 \\
\hline 15 & 11 & 28 & & 7 & 35 \\
\hline 20 & 30 & 49 & & 28 & 54 \\
\hline 30 & 32 & 4 & .39 & 46 & 36 \\
\hline 35 & 34 & 14 & 11.0 & 34 & 14 \\
\hline
\end{tabular}

Group IV: Data that change with pumping period--Continued

$I$, $J$, and pumping rate, in cubic feet per second

$\begin{array}{lrr}1621 . & 8 & 37-0.1210325 \\ 1622 . & 9 & 31-0.0654752 \\ 1623 . & 15 & 37-0.0042901 \\ 1624 . & 15 & 38-0.0034841 \\ 1625 . & 15 & 45-0.0157596 \\ 1626 . & 15 & 46-0.0407 \\ 1627 . & 16 & 46-0.0044071 \\ 1628 . & 16 & 47-0.0186424 \\ 1629 . & 17 & 42-0.0125017 \\ 1630 . & 17 & 43-0.0056616 \\ 1631 . & 17 & 45-0.0043941 \\ 1632 . & 19 & 42-0.0044851 \\ 1633 . & 20 & 10-0.0231405 \\ 1634 . & 20 & 37-0.0351007 \\ 1635 . & 20 & 42-0.0009685 \\ 1636 . & 22 & 2-0.0260306 \\ 1637 . & 22 & 10-0.0248630 \\ 1638 . & 22 & 16-0.0118562 \\ 1639 . & 23 & 2-0.0242744 \\ 1640 . & 23 & 9-0.0637013 \\ 1641 . & 23 & 12-0.0234005 \\ 1642 . & 23 & 41-0.0478410 \\ 1643 . & 24 & 2-0.0088516 \\ 1644 . & 24 & 12-0.0052001 \\ 1645 . & 25 & 2-0.0252462 \\ & & \end{array}$


Table 2.--Listing of data for 1920-70--Continued

Card

number

1646.

1647.

1648 .

1649 .

1650.

1651.

1652.

1653.

1654.

1655.

1656.

1657.

1658.

1659 .

1660 .

1661.

1662.

1663.

1664.

1665.

1666.

1667.

1668.

1669.

1670.

1671.

1672 .

1673.

1674.

1675.

1676.

1677.

1678.

1679.

1680.

1681 .

1682.

1683.

1684 .

1685.

1686.

1687.

1688 .

1689 .

1690 .

1691.

1692.

1693.

1694.

1695.
Group IV: Data that change with pumping period--Continued $\mathrm{I}, \mathrm{J}$, and pumping rate, in cubic feet per second--Continued

$27-0.0273006$

44-0.1285467

45-0.3720286

2-0.0015507

44-0.0052651

45-0.0993805

$2-0.0522078$

3-0.0384077

$28-0.0107252$

$32-0.0765374$

$45-0.1273733$

$46-0.3938854$

$27-0.0797403$

$30-0.0052001$

33-0.0057440

40-0.1031571

45-0. 1344032

46-0.5633699

45-0.5061004

46-0.2783358

47-0.1412868

7-0.0919198

33-0.0639

36-0.1387876

37-0. 1854098

45-0.1495681

46-0.1187574

47-0.0308724

48-0.4108735

6-0.1977085

7-0.2929763

33-0.0665743

37-0.0265953

42-0.1142204

44-0.1468543

45-0.2072113

46-0.3550341

47-0.4568814

48-0.2446613

7-0.0909294

33-0.0773516

37-0. 1522787

38-0.0738415

39-0.0326567

40-0.2398289

42-0. 1300027

43-0.1924040

44-0.1248026

45-0.3132870

46-0.5862243 
Card number

1696. 1697. 1698. 1699. 1700 . 1701 . 1702 . 1703. 1704 . 1705 . 1706 . 1707. 1708 . 1709 . 1710 . 1711 . 1712 . 1713 . 1714 . 1715 . 1716 . 1717 . 1718 . 1719 . 1720 . 1721. 1722 . 1723. 1724. 1725. 1726 . 1727. 1728 . 1729 . 1730 . 1731. 1732 . 1733. 1734 . 1735 . 1736 . 1737. 1738 . 1739 . 1740 . 1741 . 1742 . 1743. 1744. 1745 .
Group IV: Data that change with pumping period--Continued $I, J$, and pumping rate, in cubic feet per second--Continued

$$
\begin{array}{r}
6-0.1981947 \\
7-0.0585242 \\
14-0.0110502 \\
23-0.0142190 \\
40-0.0815604 \\
41-0.2191942 \\
42-0.1248026 \\
43-0.0648388 \\
44-0.1018896 \\
45-0.0951684 \\
46-0.5811574 \\
47-0.0293806 \\
5-0.0458727 \\
6-0.2041129 \\
7-0.1030269 \\
34-0.1082857 \\
41-0.0675201 \\
42-0.3142230 \\
43-0.2897337 \\
45-0.0163998 \\
46-0.2551432 \\
5-0.1144502 \\
6-0.0160080 \\
39-0.0380607 \\
40-0.0395858 \\
41-0.0299624 \\
45-0.0162243 \\
46-0.0383971 \\
6-0.0753651 \\
42-0.1773951 \\
43-0.0968975 \\
44-0.0091217 \\
45-0.1969801 \\
5-0.0566076 \\
6-0.0656408 \\
30-0.0732565 \\
42-0.1218775 \\
44-0.0249930 \\
45-0.4127000 \\
7-0.0778543 \\
45-0.1664977 \\
6-0.0559226 \\
7-0.0279613 \\
27-0.0030746 \\
31-0.0190454 \\
37-0.1600333 \\
43-0.0068414 \\
44-0.1292226 \\
29-0.0641563 \\
36-0.0800297 \\
\end{array}
$$


Card number

1746 . 1747. 1748 . 1749 . 1750 . 1751 . 1752 . 1753. 1754 . 1755 . 1756. 1757 . 1758 . 1759 . 1760 . 1761. 1762. 1763. 1764 . 1765 .
Group IV: Data that change with pumping period--Continued $I, J$, and pumping rate, in cubic feet per second--Continued

$\begin{array}{ll}40 & 45-0.4554574 \\ 41 & 35-0.1206587 \\ 41 & 36-0.5227896 \\ 41 & 42-0.0338007 \\ 41 & 45-0.5074817 \\ 42 & 30-0.0021255 \\ 42 & 31-0.0021255 \\ 42 & 32-0.0082879 \\ 42 & 34-0.1717823 \\ 42 & 35-0.0598012 \\ 42 & 36-0.2966361 \\ 42 & 37-0.0626166 \\ 42 & 45-0.0043161 \\ 43 & 31-0.0226634 \\ 43 & 35-0.0959809 \\ 43 & 33-0.0173944 \\ 43 & 38-0.2036492 \\ 43 & 39-0.1040509 \\ 43 & 45-0.0041113 \\ 43 & 46-0.0041276\end{array}$


Table 3.--Listing of data for 1971-77

Card number Group I: Title, Simulation options, and problem dimensions

1.

2. TER SYSTEM 1971-1977

3. WATE

4. LEAK

5. CONV

6. BLNK

7. RECH

8. SIP

9. $\quad$ CHEC

10. PUNC

11. NUME

12. $\mathrm{HEAD}$

13. NODE

14. BLNK

15. $52 \quad 55$

LARAMIE COUNTY POST-CRETACEOUS GROUND-WA

Group II: Scalar parameters

16. BLNK

17.

18.

1
10

10

$1.0 \quad 100$

0.0

Group III: Array data

Grid spacing in $X$ direction, in feet

(First card is parameter card)

19.

20.

21 .

22.

23.

24.

25.

26.

1
10560

5280

5280

5280

5280

5280

5280

1
10560
5280
5280
5280
5280
5280
5280

0
10560
5280
5280
5280
5280
5280
7920

10560

5280

5280

5280

5280

5280

7920

5280
5280
5280
5280
5280
5280
16880

5280

5280

5280

5280

5280

5280

5280

5280

5280

5280

5280

5280

5280

5280

5280

$5280 \quad 5280$

5280

Group III: Array data--Continued Grid spacing in $Y$ direction, in feet

(First card is parameter card)

0
7920

5280

5280

5280

5280

5280

5280
5280

5280

5280

5280

5280

7920

$\begin{array}{ll}7920 & 5280 \\ 5280 & 5280 \\ 5280 & 5280 \\ 5280 & 5280 \\ 5280 & 5280 \\ 5280 & 5280 \\ 7920 & 7920\end{array}$

5280
5280
5280
5280
5280
5280

5280

5280

5280

5280

5280

5280

5280

5280

5280

5280

5280

5280

5280

5280

5280

5280

5280 
Table 3.--Listing of data for 1971-77--Continued

Card

number

35.

36.

37.

38.

39.

40.

41.

42.

43.

44.

45.

46.

47.

48.

49.

50 .

51 .

52.

53.

54.

55.

56.

57.

58.

59.

60.

61 .

62.

63.

64.

65.

66.

67.

68.

69.

70.

71.

72.

73.

74.

75 .

76.

77.

78.

79.

80.

81 .

82.

\section{Group III: Array data--Continued \\ Node identification \\ (First card is parameter card)}

10

$$
\begin{array}{lllll}
10 & 10 & 10 & 10 & 10
\end{array}
$$

$10 \quad 10$

10

10

$10 \quad 10$

$\begin{array}{lllllll}15 & 15 & 15 & 10 & 10 & 10 & 10\end{array}$

15

$15 \quad 15$
15 15

$\begin{array}{llllllll}10 & 10 & 10 & 10 & 10 & 10 & 10 & 10\end{array}$

10

$10 \quad 10 \quad 10$

$10 \quad 10$ 
Table 3.--Listing of data for 1971-77--Continued

Card number
Group III: Array data--Continued Node identification--Continued

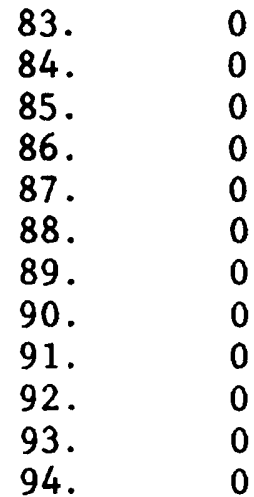

$95 . \quad 0$

96.0

$97 . \quad 0$

98. 0

$99 . \quad 0$

100. 0

$101 . \quad 0$

102. 0

$103 . \quad 0$

104. 0

105. 0

$106 . \quad 0$

$107 . \quad 0$

$108 . \quad 0$

109. 0

$110 . \quad 0$

$111 . \quad 0$

112. 0

$113 . \quad 0$

114.0

115.0

116.0

117.0

$118 . \quad 0$

119.

120.0

121.0

122.

123.

124.

125.

126.

127.

128.

129 .

130.

131.

0

0

$30 \quad 30 \quad 30 \quad 30 \begin{array}{llll}20 & 20 & 20\end{array}$

0

0

30

30

0

0 
Table 3.--Listing of data for 1971-77--Continued

Card number

132.

133.

134.

135.

136.

137.

138.

139.

140.

141.

142.

143.

144.

145.

146.

147.

148.

149.

150.

151.

152.

153.

154.

155.

156.

157.

158.

159.

160.

161.

162.

163.

164.

165.

166.

167.

168.

169.

170.

171.

172.

173.

174.

175.

176.

177.

178.

179 .

180 .
Group III: Array data--Continued

Node identification--Continued

30

30

35

$\begin{array}{llllllllll}30 & 30 & 30 & 30 & 30 & 30 & 30 & 30 & 30 & 30\end{array}$ 
Table 3.--Listing of data for 1971-77--Continued

Card

number

181.

182.

183.

184.

185 .

186.

187 .

188

189 .

190.

191.
Group III: Array data--Continued

Node identification--Continued

Explanation of node identification

192. 10 HORSE CREEK

193. 15 LITTLE HORSE CREEK

194. 20 LODGEPOLE CREEK

195. 30 CROW CREEK

196. 35 CHEYENNE MUNICIPAL DISCHARGE

197. 0

Elapsed time, in seconds and cumulative volumes, in cubic feet for mass balance

198.

199.

200 .
1608331780 . $\quad 1608331780$. $.259529507 \mathrm{E}+12 \quad 8023408640$. $.141176545 \mathrm{E}+11$
.0
201

202.

203.

204.

205.

206.

207.

208.

209.

210 .

211 .

212 .

213.
Group III: Array data--Continued

Head values for continuation of previous run, in feet

0.0

0.0

0.0

0.0

0.0

0.0

0.0

0.0

0.0

0.0

0.0

0.0

0.0

0.0

0.0

0.0

0.0

0.0

$$
0.0
$$

0.0

0.0

0.0

0.0

0.0

0.0

0.0

0.0

0.0

0.0

0.0

0.0

0.0

0.0

0.0

0.0

0.0

0.0

0.0

0.0

0.0

0.0

0.0

0.0

0.0

0.0

0.0

$118485221 \mathrm{E}+12$

0.0

5140.000

0.0

0.0

0.0

0.0

0.0

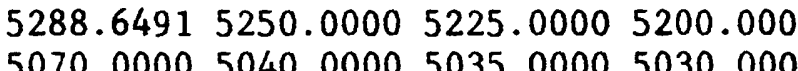

0.0

0.0

5010.00005000 .0000

035.0000

5030.00005020 .0000

0.0

0.0

0.0

0.0

0.0

0.0

0.0

0.0

0.0

0.0

0.0

0.0

0.0

0.0

5010

0.0

0.0

0.0

0.0

0.0

0.0

0.0

0.0

0.0

0.0

0.0

0.0

0.0 
Table 3.--Listing of data for 1971-77--Continued

Card number

214.

215.

216.

217.

218.

219.

220.

221.

222.

223.

224.

225 .

226.

227.

228.

229.

230 .

231.

232.

233.

234.

235.

236.

237.

238.

239.

240 .

241 .

242.

243.

244.

245.

246.

247.

248.

249.

250 .

251.

252.

253.

254 .

255.

256.

257.

258.

259.

260.

261.

262.

Group III: Array data--Continued

Head values for continuation of previous run, in feet--Continued
0.0
0.0
0.0
0.0
0.0
0.0
0.0
0.0
0.0
0.0
0.0
0.0
0.0
0.0
0.0
0.0

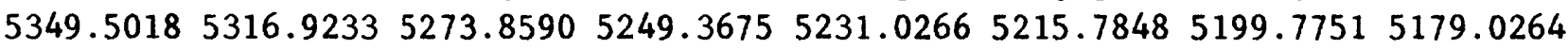
$5152.8453 \quad 5133.96265115 .86165098 .62295074 .36525048 .6437 \quad 5032.2484 \quad 5026.5993$

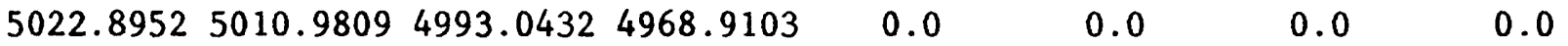
0.0
0.0
0.0
0.0
0.0
0.0
0.0
0.0
0.0
0.0
0.0
0.0
0.0

0.0

0.0

0.0

0.0

0.0

0.0

0.0

$5820.0000 \quad 5680.0000 \quad 5620.0000 \quad 5540.0000 \quad 5450.0000 \quad 5450.0000 \quad 5450.00005450 .0000$ $5424.49145399 .0635 \quad 5356.6800 \quad 5312.1895 \quad 5279.89345267 .85125254 .79295231 .8681$ $5205.68265178 .41455150 .1853 \quad 5118.7188 \quad 5090.6135 \quad 5073.3075 \quad 5058.3295 \quad 5035.0594$ $5016.51254997 .89164961 .4698 \quad 4914.5676 \quad 4890.0000 \quad 4860.0000 \quad 4836.76274808 .9714$ $4788.29154764 .90034713 .59464670 .0000 \quad 4680.0000 \quad 4740.0000 \quad 4850.00004900 .0000$ $4910.00004900 .00004850 .00004780 .0000 \quad 4750.0000 \quad 4730.0000 \quad 0.0$
0.0
0.0
0.0
0.0
0.0
0.0
0.0
0.0

$5821.5826 \quad 5696.31095626 .5955 \quad 5582.5018 \quad 5540.8425 \quad 5526.8786 \quad 5519.2600 \quad 5495.9178$ $5464.22385437 .6133 \quad 5412.97675373 .9948 \quad 5330.3363 \quad 5302.8175 \quad 5280.34695252 .4833$ $5223.07365192 .88995159 .50825124 .0438 \quad 5093.3335 \quad 5069.9760 \quad 5035.01364995 .0076$ $4970.00594954 .7894 \quad 4918.43694893 .6792 \quad 4868.55794842 .0720 \quad 4814.03474785 .7315$ $4761.5413 \quad 4729.9605 \quad 4694.4212 \quad 4685.9991 \quad 4714.3405 \quad 4787.3933 \quad 4872.96914914 .3125$ $4923.4063 \quad 4920.0973 \quad 4898.5755 \quad 4849.1216 \quad 4788.8841 \quad 4755.0000 \quad 0.0$

$\begin{array}{llllllll}0.0 & 6690.0000 & 6615.1070 & 6517.9976 & 6343.6110 & 6231.8526 & 6092.5285 & 5953.2288\end{array}$ $5828.5690 \quad 5729.26835664 .54515617 .0696 \quad 5582.6757 \quad 5562.4755 \quad 5546.32825519 .9610$ $5483.65625456 .24345431 .65125398 .6135 \quad 5359.7545 \quad 5326.2786 \quad 5294.22075258 .7518$ 5224.08865193 .00015163 .67545130 .01485100 .03195079 .80855031 .37894991 .6772 $4967.7816 \quad 4941.2679 \quad 4914.6986 \quad 4887.8603 \quad 4860.6418 \quad 4831.8904 \quad 4796.0432 \quad 4764.2238$ $4741.1765 \quad 4720.0367 \quad 4696.8090 \quad 4694.2453 \quad 4746.5368 \quad 4824.6994 \quad 4905.5353 \quad 4936.3422$ $4943.92844942 .9393 \quad 4932.7124 \quad 4902.82864840 .97504780 .0000 \quad 0.0$

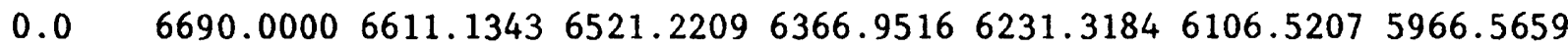
$5834.36915749 .53345690 .7695 \quad 5640.1440 \quad 5607.2800 \quad 5582.8255 \quad 5561.64315538 .2542$ $5503.74235468 .9608 \quad 5438.1132 \quad 5404.2948 \quad 5368.6652 \quad 5333.0393 \quad 5296.7295 \quad 5260.3719$ 5224.51285190 .06875175 .04045173 .78615140 .50845091 .04815037 .80244996 .3220 $\begin{array}{llllllll}4965.2770 & 4940.1360 & 4914.6475 & 4884.8460 & 4855.1295 & 4825.1560 & 4795.3787 & 4772.1124\end{array}$ $4756.4085 \quad 4747.8204 \quad 4746.7828 \quad 4752.4983 \quad 4806.33194896 .8180 \quad 4950.5193 \quad 4960.9143$ $4963.8105 \quad 4960.7073 \quad 4953.2028 \quad 4932.33104881 .63324825 .0000 \quad 0.0$

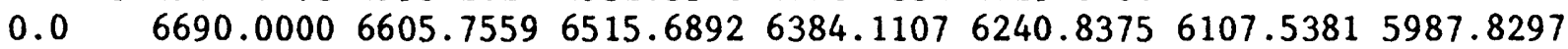
$\begin{array}{llllllll}5864.1283 & 5776.9977 & 5720.3677 & 5665.5440 & 5629.7284 & 5603.5752 & 5576.2306 & 5542.2298\end{array}$

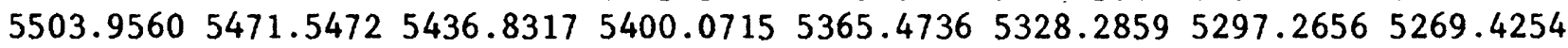
$5225.30245228 .63965219 .83905219 .5308 \quad 5173.04665111 .71515061 .02195024 .8432$ $5000.7534 \quad 4978.51124956 .8682 \quad 4938.82894904 .4217 \quad 4854.9242 \quad 4810.0391 \quad 4791.5284$ $4787.84734805 .2834 \quad 4824.6500 \quad 4850.40734895 .21884949 .7178 \quad 4998.27745015 .8513$ $5015.8048 \quad 5003.4823 \quad 4985.1365 \quad 4960.5936 \quad 4909.7564 \quad 4870.0000 \quad 0.0$

$\begin{array}{lllllllll}0.0 & 6700.0000 & 6591.9469 & 6496.2314 & 6376.3184 & 6249.1535 & 6119.9276 & 5989.2727\end{array}$ $5880.17825815 .66775753 .9316 \quad 5690.0896 \quad 5646.8142 \quad 5620.54875582 .69375541 .0685$ $5505.10935469 .55395432 .38745389 .9922 \quad 5359.97725320 .11775300 .11465294 .9236$

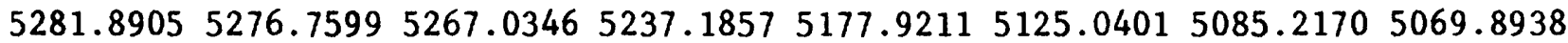
$5049.1657 \quad 5030.9738 \quad 5016.30795017 .15645004 .7538 \quad 4955.5669 \quad 4925.0648 \quad 4903.4892$ $4898.82734906 .59704918 .13454943 .77834969 .6644 \quad 5016.2354 \quad 5049.36445051 .2879$ 
Table 3.--Listing of data for 1971-77--Continued

Card number

263.

264 .

265 .

266.

267.

268.

269.

270 .

271.

272 .

273.

274.

275 .

276.

277.

278 .

279 .

280 .

281 .

282 .

283.

284 .

285 .

286 .

287.

288 .

289 .

290 .

291.

292.

293.

294.

295.

296.

297.

298.

299.

300 .

301 .

302.

303.

304.

305.

306.

307 .

308.

309 .

310 .

311 .
Group III: Array data--Continued

Head values for continuation of previous run, in feet--Continued
5045.6954
0.0

5908.4161

5499.9856

5338.9450

5108.9482

5032.3953

5050.7122

0.0

5924.8985

5540.6727

5391.6197

5205.3114

5057.4239

0.0

5974.8585

5586.1034

5428.8258

5281.495350

5189.5636

5065.8982

0.0

6043.2750

5642.43045615 .1108

5452.0332

5307.7836

5198.1776

5072.9345

0.0

6114.5068

5688.4508

5471.5883

5325.8696

5206.5276

5071.5815

0.0

5726.7775

5489.5469

5338.3700

5212.6613

5060.1613
0.0

6217.1289

5749.8966

5505.6266

5348.6865

5169.3083

$$
0.0
$$

5324.708
$4983.0590 \quad 4932.6232 \quad 4900.0000$

6453.1740

6341.63436224 .3665

0.0

6640.00006568 .9745

5720.8426

5663.02135622 .8415

6104.98515995 .7492

5470.00125439 .95315424 .5924

5401.67835377 .4796

5583.08305541 .8136

5326.44535304 .1563

5267.8623

5010.49095008 .3988

5018.1115

500.2196

5192.9833

5357.28675346 .0763

$5040.81245017 .7698 \quad 4986.3857$

5038.7930
5063.1875
4937.6384
6900.0000

5163.56365136 .1838

6590.0000

6403.8117

6317.30336209 .0096

5089.52415070 .4368

5864.9781

5799.8

5734.7798

5660.00945614 .9830

0.0

5377.7394

5503.6086

5476.6858

5450.96565431 .611554

6106.07436005 .6094

5584.92985549 .9453

5200.5225

5193.7257

5198.5903

$5199.4778 \quad 5219.1821$

5244.50895218 .5522

5044.4346

5017.0347

5092.8930

5092.2243

4932.2221

5090.2113

5081.13615069 .1082

6530.00006488 .7388

6374.2338

6279.35226197 .0107

0.0

5920.79095851 .2435

5782.0791

5727.43435683 .6843

6117.75136030 .0304

5568.20035550 .8823

5522.1679

5494.24265475 .3363

5650.24725617 .2454

5413.61045394 .4851

5365.9463

5339.73115318 .0056

5458.56495442 .5103

5276.87655274 .78955269 .9807

5149.6128

5132.9272

5265.6227

5254.4338

5299.72845283 .2868

5052.56705015 .31334966 .5562

4924.0266

5106.1854

5093.66625080 .6393

$$
0.0
$$

$6470.0000 \quad 6442.11796353 .1044 \quad 6259.98496190 .0012$

6125.17996097 .3217

5898.89635837 .8293

5787.5089

5745.756457

$5709.2135 \quad 5678.8437$

5615.11085591 .78695563 .4247

5531.85425510 .3097

5490.02545471 .4597

$\begin{array}{lll}5434.3381 & 5414.3788 & 5391.8557 \\ 5298.3961 & 5290.6759 & 5282.1633\end{array}$

5370.01365350 .6779

$5334.7458 \quad 5319.6771$

$\begin{array}{lll}5176.5950 & 5155.8129 & 5137.3298\end{array}$

5272.3959

5259.1863

5242.22685221 .0008

5121.76065109 .53345097 .65425087 .1913

$\begin{array}{lll}4918.3858 \quad 4860.0000 & 0.0\end{array}$

6400.02216345 .0325

6299.0610

6245.6280

$6189.2075 \quad 6162.7817$

6054.44985971 .01355885 .0792

5842.05625804 .4461

5766.90035727 .5772

$5564.42435537 .26225513 .6305 \quad 5492.1495$

$\begin{array}{llll}5451.7976 & 5431.5558 & 5410.6538\end{array}$

5390.23485371 .3760

5354.40865339 .1753

5314.48385303 .28265291 .9544

5279.31165264 .6913

5247.92365228 .3283

5183.2759

5160.3299

5139.4868

4912.79414855 .0000

0.0

$0.0 \quad 6430.00006410 .1959$

6341.06046302 .6083

$6251.2158 \quad 6216.1447$

$\begin{array}{lll}6116.6801 & 6037.1399 & 5942.3202 \\ 5687.7348 & 5651.3154 & 5618.6585\end{array}$

$5876.3530 \quad 5844.6980 \quad 5808.8948 \quad 5769.6511$

$\begin{array}{llll}5588.1827 & 5558.1263 & 5533.1578 & 5510.8876\end{array}$

5468.74085448 .11265427 .6978

$5407.79875388 .83645370 .8948 \quad 5353.8640$

5311.83315298 .4419

5033.1019

4995.97144951 .1366

.

6530.0000

6498.09786479 .5711

$4912.1888 \quad 4850.0000$

5088.64085078 .9064

$\begin{array}{lllll}6403.8171 & 6364.1714 & 6317.7420 & 6267.8726\end{array}$ 6156.86616067 .52845974 .9785

5909.05855864 .69985828 .92765791 .0150

5605.30995576 .24465550 .17135527 .4935

5423.29835404 .17455385 .26015365 .8242

$\begin{array}{lllll}5287.5280 & 5271.9185 & 5256.2748 & 5237.1540\end{array}$

5127.18585102 .87635084 .69835067 .8720 
Table 3.--Listing of data for 1971-77--Continued

Card number

312.

313.

314.

315 .

316 .

317.

318.

319.

320 .

321 .

322.

323.

324.

325 .

326.

327 .

328 .

329.

330 .

331 .

332.

333.

334.

335.

336.

337.

338.

339.

340 .

341 .

342 .

343.

344 .

345 .

346.

347 .

348.

349.

350 .

351 .

352 .

353.

354.

355 .

356 .

357.

358 .

359.

360 .
Group III: Array data--Continued

Head values for continuation of previous run, in feet--Continued
$5045.42675024 .52624996 .5104 \quad 4961.34964915 .75714870 .0000 \quad 0.0$

$\begin{array}{lllllllll}0.0 & 6570.0000 & 6553.2310 & 6525.3546 & 6454.5492 & 6412.3486 & 6362.8707 & 6298.5737\end{array}$ $\begin{array}{lllllllll}6245.9963 & 6180.5593 & 6093.8921 & 5994.3031 & 5921.0304 & 5880.6260 & 5840.7119 & 5801.5886\end{array}$

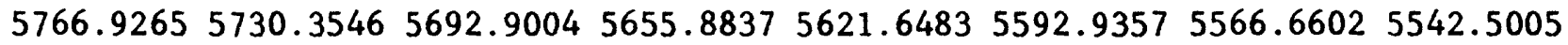
$5520.21715498 .36595477 .1473 \quad 5456.7548 \quad 5436.9302 \quad 5418.1342 \quad 5398.84945378 .4691$ $\begin{array}{lllllllll}5357.9664 & 5340.9388 & 5325.1229 & 5307.7438 & 5290.8822 & 5273.5146 & 5255.3310 & 5235.3099\end{array}$

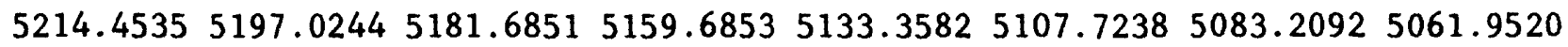
$5043.28725023 .4756 \quad 4996.6074 \quad 4963.0720 \quad 4918.00224875 .0000 \quad 0.0$

$\begin{array}{llllllll}0.0 & 6595.0000 & 6630.7101 & 6545.8329 & 6478.2758 & 6430.0634 & 6356.6516 & 6284.8806\end{array}$ $\begin{array}{lllllllll}6245.5161 & 6182.1065 & 6087.7817 & 5995.5923 & 5934.5918 & 5884.7920 & 5849.7403 & 5815.4792\end{array}$ $5780.32575744 .1676 \quad 5708.12625671 .9829 \quad 5638.1771 \quad 5610.30795584 .5566 \quad 5559.9343$ $\begin{array}{llllllll}5536.5292 & 5513.5218 & 5491.4836 & 5469.8289 & 5449.3126 & 5429.7435 & 5409.6211 & 5389.1039\end{array}$ $\begin{array}{llllllll}5369.8510 & 5350.0413 & 5331.5811 & 5313.0503 & 5294.4844 & 5275.5719 & 5255.8743 & 5234.4851\end{array}$ $5215.35185200 .99195183 .12395159 .7775 \quad 5134.7731 \quad 5108.77165081 .6435 \quad 5059.5328$ $5042.29555023 .5830 \quad 4997.5424 \quad 4964.0073 \quad 4918.6814 \quad 4870.0000 \quad 0.0$

$0.0 \quad 6625.00006649 .1006 \quad 6561.57126483 .93826410 .9744633$ $6219.98746157 .87646091 .59716015 .0676 \quad 5942.7737 \quad 5890.3749 \quad 5857.16095823 .4388$ $\begin{array}{lllllllll}5790.7340 & 5756.2805 & 5721.8936 & 5689.7548 & 5657.0064 & 5627.6290 & 5603.3433 & 5579.8311\end{array}$ $5556.34665531 .31335506 .73095483 .49215460 .83795441 .1765 \quad 5422.40245399 .5194$ $\begin{array}{lllllllll}5378.1328 & 5357.5966 & 5337.8815 & 5318.2839 & 5298.2731 & 5278.0205 & 5257.6285 & 5237.3097\end{array}$ $5218.39175202 .64855183 .43325159 .55995134 .43325105 .84465078 .2464 \quad 5059.1679$ $\begin{array}{lllllllll}5043.1532 & 5025.2100 & 4999.9370 & 4965.3508 & 4918.5041 & 4865.0000 & 0.0\end{array}$

$\begin{array}{lllllllll}0.0 & 6675.1126 & 6659.9319 & 6564.9986 & 6481.1871 & 6407.5238 & 6329.2080 & 6260.6474\end{array}$ $\begin{array}{llllllllll}6205.5351 & 6148.6496 & 6092.8797 & 6018.2989 & 5944.5827 & 5897.8931 & 5861.7726 & 5826.9991\end{array}$ $\begin{array}{lllllllll}5792.2101 & 5757.7256 & 5727.7699 & 5700.9368 & 5672.3014 & 5641.4718 & 5614.6318 & 5592.2804\end{array}$ $\begin{array}{lllllllll}5569.7791 & 5546.0062 & 5519.0972 & 5495.0935 & 5474.2184 & 5453.3350 & 5435.1890 & 5412.9477\end{array}$ $\begin{array}{llllllll}5389.0131 & 5367.3157 & 5346.7591 & 5326.3087 & 5304.7423 & 5281.8131 & 5259.4334 & 5238.5542\end{array}$ $\begin{array}{llllllll}5218.6680 & 5199.8215 & 5179.4060 & 5157.3279 & 5133.7030 & 5106.2383 & 5079.4205 & 5060.5976\end{array}$

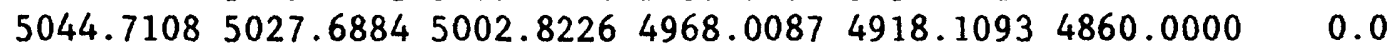

$\begin{array}{lllllll}0.0 & 6706.1779 & 6669.7211 & 6575.1007 & 6475.5956 & 6407.0590 & 639\end{array}$ $6193.55326139 .72946082 .29096008 .4895 \quad 5945.6171 \quad 5899.7275$ $5789.91715757 .6806 \quad 5731.87295705 .4142 \quad 5678.5393 \quad 5651.1459$ $5574.77495552 .79275528 .5746 \quad 5504.4013 \quad 5482.5652 \quad 5462.2659$

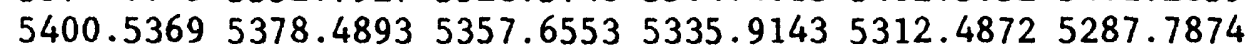
$5216.1426 \quad 5195.3410 \quad 5174.4348 \quad 5153.6019 \quad 5133.3546 \quad 5111.4220508$ $5050.23565029 .9079 \quad 5004.0348 \quad 4968.6088 \quad 4918.6660 \quad 4860.0000$ $\begin{array}{llllll}0.0 & 6720.9137 & 6677.0876 & 6578.9995 & 6477.5816 & 6410.2415\end{array}$ 6331.60576255 .5524 5861.43745826 .9284 5623.25955597 .4330 5444.45395424 .2185 5261.69615237 .6159 5088.58725069 .1469 0.0 $6190.64686130 .9346 \quad 6058.4436 \quad 5988.3013 \quad 5949.8923 \quad 5906.1871 \quad 5864.1717 \quad 5826.8800$ $5789.88735758 .76865734 .01985708 .80235683 .27345656 .69745628 .4713 \quad 5603.6605$ $\begin{array}{llllllllll}5584.4956 & 5562.5072 & 5537.5270 & 5514.1610 & 5492.3151 & 5471.4732 & 5451.5768 & 5431.2715\end{array}$ $5409.28995387 .47865366 .07175344 .0211 \quad 5320.0322 \quad 5294.2350 \quad 5263.9386 \quad 5234.4155$ $\begin{array}{llllllll}5210.3971 & 5188.2338 & 5166.3097 & 5148.3883 & 5131.9940 & 5114.5391 & 5097.2368 & 5080.8358\end{array}$ 5064.2193 5042.5786 0.0 6732.3270668 6185.1078 6133.1081 5011.77094967 .50064917 .50274865 .0000 0.0 $6068.25865996 .9735 \quad 5940.94875896 .3248 \quad 5859.3888 \quad 5824.1660$ $\begin{array}{lllllllll}5790.0258 & 5760.9640 & 5736.1849 & 5712.3434 & 5687.7170 & 5662.4761 & 5637.1734 & 5613.0216\end{array}$ $\begin{array}{llllllll}5591.6401 & 5570.1660 & 5547.1930 & 5524.5534 & 5502.8868 & 5481.8296 & 5460.5741 & 5438.9133\end{array}$ 5416.75005394 .94755373 .21575350 .01665324 .86345296 .81815264 .20145229 .8940 5200.26915177 .93905157 .97585140 .52925123 .76825109 .23865096 .43715084 .0912 
Table 3.--Listing of data for 1971-77--Continued

Card

number

361.

362.

363.

364.

365 .

366 .

367.

368.

369 .

370 .

371 .

372.

373.

374.

375 .

376.

377.

378 .

379 .

380 .

381 .

382 .

383 .

384 .

385 .

386.

387.

388 .

389 .

390.

391.

392.

393.

394.

395.

396.

397.

398.

399.

400.

401 .

402 .

403.

404.

405 .

406.

407.

408.

409 .
Group III: Array data--Continued

Head values for continuation of previous run, in feet--Continued
$5071.45565053 .0693 \quad 5017.8938 \quad 4967.4713 \quad 4916.4351 \quad 4865.0000 \quad 0.0$

$\begin{array}{lllllllll}0.0 & 6733.3435 & 6666.3711 & 6575.5806 & 6501.7829 & 6431.7866 & 6348.2896 & 6257.2975\end{array}$

$6191.6863 \quad 6144.5148 \quad 6083.6674 \quad 6003.9772 \quad 5936.5258 \quad 5888.4696 \quad 5851.3992 \quad 5820.5637$

$\begin{array}{lllllllll}5787.3930 & 5761.8374 & 5738.7422 & 5715.3790 & 5691.4150 & 5666.9356 & 5643.3759 & 5621.4566\end{array}$

$\begin{array}{lllllllll}5600.0393 & 5578.0741 & 5555.5678 & 5533.3719 & 5511.9575 & 5491.1892 & 5468.7762 & 5445.8309\end{array}$

5422.87265400 .19025378 .22065354 .03975327 .45145296 .10375258 .75225220 .4598

$\begin{array}{lllllllll}5188.3170 & 5165.1341 & 5144.5842 & 5126.1523 & 5110.7998 & 5099.8747 & 5090.5001 & 5080.2793\end{array}$

$5069.08945050 .8335 \quad 5015.4596 \quad 4964.9036 \quad 4914.07914860 .0000 \quad 0.0$

$\begin{array}{lllllllll}0.0 & 6700.0000 & 6658.8628 & 6576.4464 & 6509.7841 & 6447.7079 & 6354.1481 & 6253.4163\end{array}$

$6198.47716145 .54996089 .4426 \quad 6010.42345935 .7767 \quad 5888.4655 \quad 5849.0962 \quad 5812.1641$

$5780.85715761 .57245740 .24365717 .91315694 .5157 \quad 5669.10865645 .8140 \quad 5626.4720$

$\begin{array}{llllllllll}5605.8149 & 5583.4503 & 5560.6393 & 5538.5008 & 5517.5890 & 5498.8480 & 5476.4640 & 5450.7282\end{array}$

$\begin{array}{llllllllll}5426.5574 & 5403.4732 & 5380.8150 & 5355.5654 & 5327.1650 & 5292.7196 & 5250.7597 & 5208.0657\end{array}$

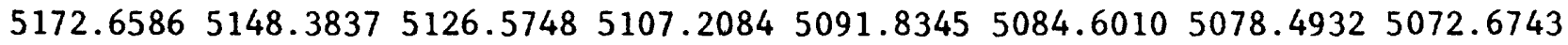

$5059.6276 \quad 5038.2908 \quad 5005.4152 \quad 4959.2645 \quad 4910.5428 \quad 4860.0000 \quad 0.0$

$\begin{array}{llllllll}0.0 & 6660.0000 & 6650.3645 & 6581.1230 & 6511.1766 & 6459.1429 & 6377.8906 & 6269.5624\end{array}$ $\begin{array}{llllllll}6201.4640 & 6146.4388 & 6080.2902 & 5995.8296 & 5923.5544 & 5875.6287 & 5839.3907 & 5808.6679\end{array}$ $\begin{array}{llllllll}5782.3653 & 5762.0902 & 5741.4361 & 5720.1574 & 5698.2106 & 5675.4989 & 5652.9988 & 5632.6238\end{array}$ $5613.21165590 .68645565 .64115542 .2642 \quad 5520.54045500 .63945477 .89395452 .0628$ $\begin{array}{lllllllll}5428.9658 & 5406.9842 & 5382.5452 & 5353.9382 & 5321.9620 & 5283.9587 & 5237.8407 & 5188.6255\end{array}$ $\begin{array}{lllllllll}5150.9371 & 5128.1665 & 5104.7648 & 5086.4373 & 5072.7749 & 5064.8852 & 5058.8529 & 5054.7082\end{array}$ $\begin{array}{llllllll}5040.2321 & 5021.0955 & 4991.2933 & 4949.5122 & 4904.7330 & 4850.0000 & 0.0\end{array}$

$\begin{array}{llllllllll}0.0 & 6630.0000 & 6626.7590 & 6582.1380 & 6533.8936 & 6486.2991 & 6384.7350 & 6257.9578\end{array}$ $\begin{array}{lllllllll}6206.1766 & 6149.6594 & 6069.7891 & 5976.2467 & 5910.8123 & 5867.2389 & 5836.8385 & 5809.2459\end{array}$ $5783.14055761 .69455741 .95065721 .7485 \quad 5701.3206 \quad 5680.2576 \quad 5659.0607 \quad 5638.5426$ $\begin{array}{lllllllll}5619.1797 & 5596.2922 & 5569.4601 & 5544.5161 & 5521.4493 & 5499.6578 & 5475.1980 & 5448.0942\end{array}$ $\begin{array}{llllllllll}5424.8179 & 5402.9268 & 5377.9810 & 5347.3897 & 5312.6185 & 5271.6503 & 5223.3257 & 5172.2442\end{array}$ $\begin{array}{llllllll}5135.6035 & 5112.3696 & 5087.3002 & 5071.2478 & 5059.1958 & 5046.1838 & 5035.8594 & 5029.9091\end{array}$ $5019.22995001 .5170 \quad 4972.6899 \quad 4934.8970 \quad 4898.8579 \quad 4840.0000 \quad 0.0$

$\begin{array}{llllllll}0.0 & 0.0 & 6615.0000 & 6569.1400 & 6517.4086 & 6486.9629 & 6393.6230 & 6283.9295\end{array}$ $6210.9030 \quad 6133.62946047 .94825964 .6470 \quad 5903.4827 \quad 5863.6752 \quad 5835.47115807 .5692$ $\begin{array}{llllllll}5782.9007 & 5762.0727 & 5741.6233 & 5722.2668 & 5703.9901 & 5684.0773 & 5663.1010 & 5641.9970\end{array}$ $5621.57885597 .64625569 .07015543 .4602 \quad 5519.2631 \quad 5494.71275468 .81985442 .9599$ $\begin{array}{llllllllll}5418.4999 & 5394.2800 & 5369.1848 & 5340.9584 & 5305.2142 & 5262.1314 & 5216.1080 & 5172.1280\end{array}$ $5138.91245111 .46745084 .95415067 .6152 \quad 5049.8348 \quad 5033.15375017 .53205007 .1289$ $4996.33194978 .5245 \quad 4953.5088 \quad 4923.4471 \quad 4897.3266 \quad 4860.0000 \quad 0.0$

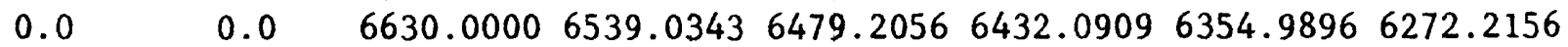
$6207.78426129 .2952 \quad 6030.0990 \quad 5963.3479 \quad 5904.1427 \quad 5865.0675 \quad 5833.26795805 .0088$ $\begin{array}{lllllllll}5782.1622 & 5761.6832 & 5739.9255 & 5720.8740 & 5706.2761 & 5687.8804 & 5666.3529 & 5643.5277\end{array}$ $5619.90685595 .6660 \quad 5569.72825543 .04125516 .4187 \quad 5490.3917 \quad 5464.4076 \quad 5438.5256$ 5413.11835388 .39735364 .02275336 .63055301 .76155261 .56745218 .61455177 .5156 $5146.63915121 .48995092 .98825065 .09605040 .39055020 .66065001 .7594 \quad 4992.5476$ $4981.69874968 .7233 \quad 4949.9816 \quad 4927.5293 \quad 4900.3321 \quad 4870.0000 \quad 0.0$
0.0
0.0
0.0
6485.00006413 .5152634
6344.77156294 .97066251 .7457 $6194.70116125 .36976039 .99615959 .5891 \quad 5903.93615866 .5840 \quad 5833.4407 \quad 5804.4992$

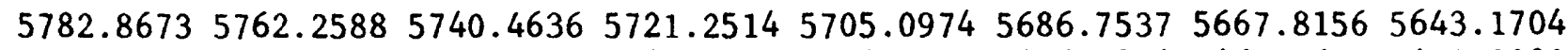
$5617.11705594 .49015569 .19445542 .49165514 .1673 \quad 5486.18245460 .06115433 .9080$ $\begin{array}{lllllllll}5407.6599 & 5382.6048 & 5358.4008 & 5332.4891 & 5297.6168 & 5259.2750 & 5221.7775 & 5186.1381\end{array}$ 5157.38625132 .08115103 .12255071 .15495042 .46655019 .30285002 .54914994 .1166 
Table 3.--Listing of data for 1971-77--Continued

Card number

410.

411.

412.

413.

414.

415 .

416.

417.

418 .

419.

420 .

421.

422.

423.

424.

425 .

426.

427.

428.

429 .

430.

431.

432.

433.

434.

435.

436.

437.

438.

439.

440 .

441 .

442.

443.

444.

445 .

446.

447.

448 .

449 .

450 .

451 .

452 .

453.

454.

455 .

456.

457.

458 .
Group III: Array data--Continued

Head values for continuation of previous run, in feet--Continued
4983.61894970 .34614950 .5694

$$
0.0
$$

0.0

0.0

4933.8049
6475.0000

4955.9988

6179.99776118 .90696047 .1133

5786.03015763 .43315742 .6803

5620.18715595 .60625569 .2715

$5402.9070 \quad 5377.9455 \quad 5355.9380$

5166.9525

4991.3286

0.0

5137.6114
4989.9394

5107.9422

0.0

0.0

5960.6446

6400.0148
5903.3533

4930.0000

0.0

6155.00586100 .46166047 .1442

$5789.8307 \quad 5766.8758 \quad 5747.5250$

5624.16665598 .82855567 .8065

5400.24355375 .45335353 .7020

5172.10525142 .12805111 .1908

5055.13625051 .37395048 .9227

0.0

0.0

0.0

5724.5692
5542.9404

5705.9256

6325.0121

$6265.0152 \quad 6225.1057$

5833.90185807 .5284

6144.98786087 .84006035 .7158

$5798.4380 \quad 5775.3080 \quad 5753.9404$

5624.57695598 .75945566 .1800

$5398.95675374 .0680 \quad 5352.0982$

5175.37845145 .50115113 .1207

5122.01585097 .3342

0.0

0.0

5084.2276

6655.0000

6151.85496069 .8975

6016.6434

5327.6253

5076.8757

4980.2033

6480.0000

5963.9842

5727.8285

5534.5927

5324.4928

5080.5394

5033.2498

6515.0000

5960.8580

5732.6991

5530.7837

5321.9376

5077.5958

5063.0685

6587.9474

5963.7975

5760.8559

5738.9466

5805.33265783 .2045

5564.9930

5530.4542

5621.73245595 .3977

5351.6640

5322.5628

5184.2420

5151.9780

5115.8042

5081.1102

5130.71615121 .1445

0.0

0.0

6740.00006634 .6997

6162.97126057 .6899

5810.34945790 .2424

5620.47155595 .0622

5400.38715376 .3712

5192.86925166 .4571

5148.43755143 .3042

0.0

0.0

6188.66886085 .5405

5810.55115788 .3879

5620.04915599 .7752

5400.44375379 .5152

5197.78755174 .8028

5159.92695154 .7995

$$
0.0 \quad 0.0
$$

6214.20336109 .3619

5808.95625784 .7234

5615.89415590 .0532

5398.06605377 .6633

6010.0546

6634.6997
5969.9700

5765.1323

5744.9294

5566.3715

5535.5027

5350.3419

5319.2817

5131.9835

5091.4504

6850.0000

5103.0841

6020.8262

6669.2862

5764.7094

5569.7808

5979.8559

5741.4366

5540.1060

5352.12115317 .3103

5144.41395105 .0123

5142.10635119 .8658

6890.0000

6702.6510

6034.0820

5737.5439

5560.27595527 .2138

5349.2003

5319.8937

5201.07455181 .2621

\subsection{9}

5046.1033

5007.0960

6413.3493

5901.5337

5707.4295

5502.9716

5286.7694

5051.0438

5019.7760

6446.4818

5901.7548

5710.4517

5500.0488

5287.2443

5054.3588
5034.7719

6469.6753

5913.4590

5499.0496

5289.3509

5059.8726

5052.7067

6505.2942

5719.9524

5500.7406

5289.5554

5068.6836

5070.6255

6537.4339

5930.0722

5716.5610
5503.3316

5288.1248

5082.9837

5087.3629

6543.2612

5713.4919

5497.8622

5292.3416

5095.8358
5715.1088

5686.1695

5480.7391

5255.2416

5023.9268

4950.0000

6351.4007

5866.7473

5686.2686

5478.9257

5255.3865

5033.7363

4975.0000

6359.5452

5870.8988

5686.2059

5476.7385

5259.8603

5047.4378

5000.0000

6396.2264

5875.1642

5691.0879

5474.7261

5262.0930

5055.2306

5025.0000

6440.9566

665.68705643 .4950

5456.53365430 .5493

5227.44645197 .7033

5008.99174999 .2065

$$
0.0
$$

6279.17656221 .8095

$5837.8930 \quad 5814.0909$

5664.96775644 .2627

5454.25295428 .2034

5229.59435201 .0786

5021.47765048 .6045 0.0

$6307.8246 \quad 6234.5488$

5845.10925821 .6013

5663.35035644 .1610

5452.24185426 .4926

5234.02995204 .6163

5043.26625093 .1279

0.0

6337.06186262 .0096

5852.46965828 .0849

5667.19155644 .5511

5450.40345425 .3535

5237.83215211 .6749

5065.49625104 .8895 0.0

6373.24256284 .8737 $5890.23395865 .1115 \quad 5835.3092$ 5694.97495670 .01345645 .5468 $5471.92815448 .5213 \quad 5424.5914$ $5265.7760 \quad 5241.8830 \quad 5218.2213$ 5061.47285088 .78465127 .6564 $5040.0000 \quad 0.0$ 
Table 3.--Listing of data for 1971-77--Continued

Card number

459.

460.

461 .

462.

463.

464.

465 .

466.

467.

468 .

469.

470 .

471 .

472 .

473 .

474.

475 .

476 .

477 .

478.

479 .

480 .

481 .

482 .

483.

484.

485 .

486 .

487 .

488 .

489 .

490 .

491.

492.

493.

494.

495.

496.

497.

498.

499.

500 .

501 .

502.

503.

504.

505.

506.

507.
Group III: Array data--Continued

Head values for continuation of previous run, in feet--Continued
$5165.58195161 .01035149 .67845130 .09615101 .02185065 .0000 \quad 0.0$

$\begin{array}{lllllllll}0.0 & 7140.0000 & 6884.6628 & 6706.9986 & 6566.1022 & 6489.3011 & 6406.0579 & 6321.3193\end{array}$

$\begin{array}{llllllllll}6221.6743 & 6117.1940 & 6042.3993 & 5989.7988 & 5928.1680 & 5874.3191 & 5845.2289 & 5826.7903\end{array}$ $5805.46795780 .91495756 .7758 \quad 5733.5721 \quad 5709.5300 \quad 5685.2247 \quad 5661.6200 \quad 5636.8168$ $5609.23685580 .81065546 .12945507 .3657 \quad 5472.9162 \quad 5440.3232 \quad 5417.9990 \quad 5400.7895$ $\begin{array}{lllllllll}5388.5980 & 5370.1096 & 5343.5600 & 5317.1766 & 5291.4948 & 5267.2124 & 5242.3397 & 5220.9236\end{array}$ 5203.80615185 .89925158 .40795126 .82685110 .21365118 .48645154 .71845172 .6106 $5170.39085166 .09685156 .52805139 .67005112 .84515070 .0000 \quad 0.0$

$\begin{array}{lllllllll}0.0 & 7110.0000 & 6897.8404 & 6709.6978 & 6578.7528 & 6496.9683 & 6409.9579 & 6312.2099\end{array}$ $6205.54596108 .76826048 .2166 \quad 5994.7850 \quad 5922.6127 \quad 5867.5005 \quad 5840.9414 \quad 5823.2371$ $\begin{array}{lllllllll}5801.6242 & 5777.1098 & 5752.8862 & 5728.9607 & 5704.2630 & 5678.8550 & 5653.4802 & 5626.9662\end{array}$ $\begin{array}{llllllll}5598.6026 & 5564.6672 & 5519.5102 & 5472.9385 & 5433.3794 & 5406.8219 & 5395.2873 & 5382.8433\end{array}$ $\begin{array}{llllllll}5374.8993 & 5358.3473 & 5334.2893 & 5310.2958 & 5285.7961 & 5262.8361 & 5241.8708 & 5223.1579\end{array}$ $\begin{array}{llllllll}5205.5591 & 5188.2381 & 5162.4060 & 5136.5590 & 5124.0889 & 5133.8495 & 5160.9923 & 5177.6682\end{array}$ $5175.47335171 .35315162 .56095147 .98465123 .52135080 .0000 \quad 0.0$

$\begin{array}{llllllll}0.0 & 7080.0000 & 6889.7159 & 6696.8183 & 6579.5822 & 6508.3286 & 6399.6086 & 6266.4773\end{array}$ $\begin{array}{lllllllll}6176.0288 & 6103.8286 & 6045.5686 & 5986.2507 & 5911.1135 & 5865.2161 & 5839.3160 & 5820.4085\end{array}$ $5798.37375773 .5963 \quad 5748.4345 \quad 5723.5599 \quad 5697.6718 \quad 5670.9489 \quad 5643.9114 \quad 5614.3133$ $\begin{array}{lllllllll}5582.9530 & 5544.8532 & 5492.7274 & 5440.0012 & 5401.4128 & 5382.5910 & 5374.6171 & 5367.6046\end{array}$ $\begin{array}{llllllll}5358.7318 & 5342.2739 & 5321.1900 & 5300.2058 & 5279.7236 & 5260.8139 & 5244.4633 & 5226.0319\end{array}$

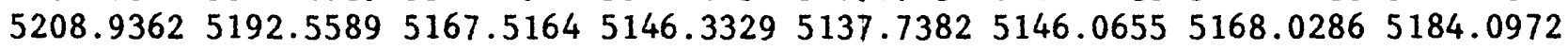
$\begin{array}{lllllll}5182.3466 & 5177.3685 & 5169.1150 & 5155.3749 & 5133.2279 & 5090.0000 & 0.0\end{array}$

$\begin{array}{lllllllll}0.0 & 7100.0000 & 6854.1022 & 6680.7233 & 6565.6170 & 6491.6712 & 6353.3925 & 6219.1741\end{array}$ $\begin{array}{llllllll}6145.0629 & 6087.5636 & 6039.0903 & 5979.3098 & 5902.2089 & 5863.2556 & 5838.5802 & 5818.9782\end{array}$ $\begin{array}{lllllllll}5795.8628 & 5769.7279 & 5743.4191 & 5717.0923 & 5689.7649 & 5661.1758 & 5631.4827 & 5597.4757\end{array}$ $\begin{array}{llllllll}5563.3158 & 5524.9483 & 5473.4099 & 5423.2680 & 5388.4253 & 5372.3166 & 5362.6600 & 5353.7516\end{array}$

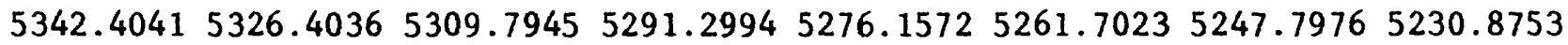
$\begin{array}{llllllll}5212.0976 & 5193.3096 & 5171.4783 & 5156.5224 & 5151.6569 & 5155.7978 & 5173.5055 & 5215.5244\end{array}$ $\begin{array}{lllllll}5190.8016 & 5185.1780 & 5176.4477 & 5162.7462 & 5142.3126 & 5100.0000 & 0.0\end{array}$

$\begin{array}{lllllllll}0.0 & 7050.0000 & 6807.2431 & 6643.7398 & 6535.0881 & 6427.1475 & 6265.3044 & 6165.6983\end{array}$ $6095.14176055 .5160 \quad 6028.6715 \quad 5969.6968 \quad 5903.6693 \quad 5865.1580 \quad 5841.7199 \quad 5819.4650$ $\begin{array}{llllllllll}5792.8874 & 5763.6502 & 5736.3738 & 5709.2435 & 5680.8769 & 5650.6668 & 5615.6128 & 5575.7017\end{array}$ $\begin{array}{llllllllll}5539.2378 & 5500.9556 & 5457.2666 & 5411.4831 & 5378.1181 & 5364.7828 & 5353.5973 & 5342.3688\end{array}$ $\begin{array}{lllllllll}5330.1189 & 5315.8284 & 5301.9622 & 5284.9271 & 5271.3688 & 5260.4912 & 5246.9042 & 5230.6694\end{array}$ $\begin{array}{llllllll}5213.5199 & 5195.3136 & 5176.2800 & 5164.5491 & 5162.0700 & 5164.0536 & 5180.7366 & 5233.0110\end{array}$ $5204.80325194 .46875183 .73575170 .07025150 .5545 \quad 5120.0000 \quad 0.0$

$0.0 \quad 7000.00006750 .03526594 .30726470 .69256348 .97016205 .05626106 .1167$ $6030.75116007 .4562 \quad 5994.77695948 .8145 \quad 5901.8844 \quad 5868.2995 \quad 5847.5598 \quad 5820.9755$ $5789.82975758 .47145729 .88365701 .3326 \quad 5670.22045632 .08355583 .28635542 .0131$ 5506.98195472 .94965442 .20135406 .58475375 .23215356 .04105343 .00245331 .1113 5317.34715303 .64775289 .59725275 .02065264 .61175253 .61795240 .81815228 .4805 $5213.31945195 .37145179 .09055171 .06765169 .43285170 .20375186 .9515 \quad 5240.9074$ $5214.25545203 .60435190 .20475176 .94445157 .40245125 .0000 \quad 0.0$

$\begin{array}{llllllll}0.0 & 6900.0000 & 6707.9097 & 6536.4584 & 6377.4710 & 6266.9876 & 6147.2408 & 6038.8869\end{array}$ $5971.98095956 .91815956 .28055931 .02965894 .04105871 .8307 \quad 5851.9295 \quad 5827.4760$ $5789.4507 \quad 5749.02835719 .60195685 .2055 \quad 5643.40365593 .4942 \quad 5544.76835512 .2811$ $5477.42285447 .4928 \quad 5425.01245395 .8193 \quad 5369.1785 \quad 5346.59065331 .07005316 .9540$ $\begin{array}{lllllllll}5301.7770 & 5287.8882 & 5274.4344 & 5262.9716 & 5255.2159 & 5245.4338 & 5235.5297 & 5225.8632\end{array}$ $5212.62195196 .08785181 .44205175 .65665174 .72835175 .55235192 .5675 \quad 5246.1323$ 
Table 3.--Listing of data for 1971-77--Continued

Card number

508.

509.

510 .

511.

512.

513.

514.

515 .

516.

517.

518.

519.

520 .

521 .

522 .

523.

524.

525 .

526.

527.

528 .

529 .

530 .

531.

532.

533.

534.

535.

536.

537.

538.

539.

540 .

541.

542.

543.

544.

545.

546.

547.

548 .

549.

550 .

551 .

552 .

553.

554.

555.

556 .
Group III: Array data--Continued

Head values for continuation of previous run, in feet--Continued
5220.7219
0.0

5921.8688

5780.9546

5446.2486

5283.2986

5211.4528

5225.9454

0.0

5850.1135

5755.3002

5418.4110

5259.3520

5211.5210

5230.5115

0.0

5794.1510

5697.8840

5397.9077

5231.0647
5209.7172

5235.1372

0.0

5760.7558

5611.1254

5369.1460

5206.2256

5201.4430

5244.796

0.0

5731.2519

5538.5446

5328.9071

5182.0787

5171.8182

5252.0991

0.0

5692.940

5470.0456

$5285.3917 \quad 5267.5769 \quad 5254.6715$

5152.83035142 .67825133 .6902

5120.63535150 .88735175 .7552

5252.8483

0.0

$5244.6840 \quad 5216.5472$
6350.0000
6200.0000

5630.00005610 .0000

5260.00005235 .00005220 .0000

5115.00005105 .00005095 .0000

5070.00005065 .00005090 .0000

5217.39955197 .6022
5183.46855165 .44625140 .0000

6323.3373

6232.5462

5892.07705873 .5766

5910.4453

5611.2356

5564.8534

5376.57575353 .60815330 .8564

5248.8153

5239.2334

5229.0551

5178.5525

5178.2823

5180.7065
5150.0000

5187.0069
6416.80496276 .06526176 .2144

$5882.55365879 .7249 \quad 5864.4986$

$5623.9125 \quad 5578.74205533 .8769$

5354.11035333 .20835308 .6550

5230.58775219 .0335521

210.4762

5187.7275

5186.6730

5189.4594

5188.67675174 .30935160 .0000

0.0

6111.01375984 .9854

$5850.6245 \quad 5820.5886$

5521.01765485 .1700

$5312.8400 \quad 5295.2259$

$5224.8498 \quad 5219.1174$

$5198.4355 \quad 5251.4717$

0.0

6050.92775928 .9248

5839.30435802 .7299

5490.40405454 .0170

$5286.7210 \quad 5269.8449$

5210.78715210 .2429

5206.92155258 .5696 0.0

6363.69176213 .76426102 .67015984 .10945865 .9393 $\begin{array}{lllll}5852.94915860 .1685 & 5847.2030 & 5818.8990 & 5772.0104\end{array}$ $\begin{array}{lllll}5557.0263 & 5532.2172 & 5495.9783 & 5462.7200 & 5428.9936\end{array}$ $\begin{array}{llllll}5328.7527 & 5305.3611 & 5277.3142 & 5252.9629 & 5239.5260\end{array}$ $5211.22045200 .1325 \quad 5194.19185198 .12395201 .7768$ $\begin{array}{lllll}5205.9051 & 5204.5269 & 5206.9999 & 5252.5475 & 5272.4235\end{array}$ $5189.56085175 .02615150 .0000 \quad 0.0$

6296.98076139 .59736027 .12715912 .44925804 .9251 5802.61705821 .58225809 .76465778 .60575708 .6534 $\begin{array}{llllll}5485.4385 & 5470.7355 & 5445.5879 & 5421.7869 & 5392.0204\end{array}$ 5302.22825275 .55275245 .65875222 .29725212 .1689 $5199.83475192 .27845186 .74495177 .1203 \quad 5170.0925 \quad 5174.6398 \quad 5187.9203$

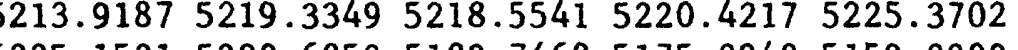

5189.74685175 .09405150 .0000

5251.29345258 .3545 0.0 $\begin{array}{lllllll}6224.5714 & 6074.2849 & 5972.1159 & 5862.7680 & 5765.7487 \\ 5726.9492 & 5732.3356 & 5715.4464 & 5681.5398 & 5615.6486\end{array}$

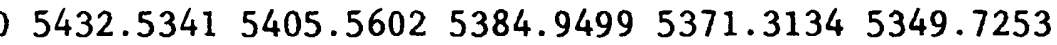
15274.92005246 .27645219 .76085199 .81175189 .1320 825156.93465150 .36445140 .45175133 .44585144 .6390 85218.25235225 .93275233 .83115254 .96675260 .3924 $95188.58885174 .51715150 .0000 \quad 0.0$

$6139.33895994 .90025904 .65645803 .3243 \quad 5722.3019$ $\begin{array}{lllll}5653.8435 & 5634.3874 & 5605.9392 & 5563.7082 & 5512.0490\end{array}$

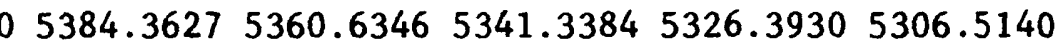
$\begin{array}{llllll}5234.7847 & 5210.3621 & 5191.7065 & 5175.1650 & 5163.4967\end{array}$ 5124.41715117 .94725110 .03135101 .04775097 .4540 5199.10985222 .00175243 .48435259 .70585262 .0645 $5188.39965173 .20295150 .0000 \quad 0.0$

6030.00005900 .00005820 .00005750 .00005690 .0000 5590.00005570 .00005550 .00005510 .00005460 .0000 5350.00005325 .00005305 .00005290 .00005275 .0000 $5205.00005185 .00005170 .00005145 .0000 \quad 5130.0000$ 5085.00005080 .00005075 .00005070 .00005070 .0000 5140.00005190 .00005245 .00005250 .00005250 .0000 
Table 3.--Listing of data for 1971-77--Continued

Card number

557.

558 .

559 .

560 .

561.

562 .

563.

564.
Group III: Array data--Continued

Head values for continuation of previous run, in feet--Continued

$\begin{array}{ccccc}5250.0000 & 5250.0000 & 5210.0000 & 5190.0000 & 5170.0000 \\ 0.0 & 0.0 & 0.0 & 0.0 & 0.0 \\ 0.0 & 0.0 & 0.0 & 0.0 & 0.0 \\ 0.0 & 0.0 & 0.0 & 0.0 & 0.0 \\ 0.0 & 0.0 & 0.0 & 0.0 & 0.0 \\ 0.0 & 0.0 & 0.0 & 0.0 & 0.0 \\ 0.0 & 0.0 & 0.0 & 0.0 & 0.0 \\ 0.0 & 0.0 & 0.0 & 0.0 & 0.0 \\ & & & & \\ & \text { Group III: Array data--Continued } \\ \text { Starting head matrix, in feet }\end{array}$

565.

566.

567.

568 .

569.

570 .

571.

572 .

573.

574 .

575.

576.

577.

578.

579.

580 .

$\begin{array}{lll}1 & & 1 \\ & 0.0 & \\ & 0.0 & \\ & 0.0 \\ & 0.0 \\ & & \\ & 0.0 \\ & 0.0 \\ & 0.0 \\ & 0.0\end{array}$

1

$\begin{array}{ll} & 2 \\ 0.0 & \\ 0.0 \\ 0.0 \\ 0.0 \\ 0.0 \\ 0.0 \\ 0.0 \\ 0.0\end{array}$

0.0

0.0

0.0

0.0

0.0

0.0

0.0

0.0

0.0

0.0

0.0

0.0

0.0

0.0

0.0

0.0

0.0

0.0

0.0

0.0

0.0

0.0

0.0

0.0

0.0

0.0

0.0

0.0

0.0

0.0

0.0

0.0

0.0

0.0

0.0

0.0

0.0

0.0

0.0

0.0

0.0

$0.0 \quad 0.0$

0.0

0.0

0.0

0.0

$5288.65405250 .00005225 .00005200 .00005170 .0000 \quad 5140.0000 \quad 5130.00005115 .0000$ 5070.00005040 .00005035 .00005030 .00005020 .00005000 .00005000 .00005010 .0000

$$
5010
$$

$$
0.0
$$

0.0

0.0

0.0

0.0

0.0
0.0

0.0

0.0

0.0

0.0

0.0

0.0

0.0

0.0

0.0

0.0

0.0

0.0

0.0

0.0

0.0

0.0

0.0

0.0

0.0

0.0

0.0

0.0

582.

$5349.51475316 .9350 \quad 5273.86875249 .37685231 .0355 \quad 5215.79445199 .78415179 .0356$

583.

584.

585 .

586.

5152.85335133 .97095115 .87075098 .63225074 .37405048$.

5022.90165010 .99274993 .06094968 .9274

$$
0.0
$$

0.0

0.0

0.0

0.0

0.0

0.0

0.0

0.0

0.0

0.0

0.0

$5820.00005680 .00005620 .00005540 .0000 \quad 5450.0000 \quad 5450.00005450 .0000 \quad 5450.0000$

0.0

0.0

588.

589.

590.

591.

592.

593.

594.

595.

596.

597.

598.

599.

$5424.50755399 .08295356 .69875312 .2055 \quad 5279.9076 \quad 5267.86525254 .80595231 .8783$

5205.69055178 .42145150 .19375118 .72695090 .62215073 .31765058 .33465035 .0641

$5016.5245 \quad 4997.9448 \quad 4961.5284 \quad 4914.6065 \quad 4890.0000 \quad 4860.0000 \quad 4836.8024 \quad 4809.0417$

$4788.2560 \quad 4764.9373 \quad 4713.6958 \quad 4670.0000 \quad 4680.0000 \quad 4740.0000 \quad 4850.0000 \quad 4900.0000$ 4910.0000490

$$
0.0
$$

0.0

0.0

0.0

0.0

0.0

0.0

$\begin{array}{llllllll}5821.5984 & 5696.3263 & 5626.6082 & 5582.5143 & 5540.8561 & 5526.8938 & 5519.2775 & 5495.9366\end{array}$ $5464.24525437 .63595412 .9993 \quad 5374.0155 \quad 5330.3540 \quad 5302.8316 \quad 5280.35645252 .4845$ 5223.06685192 .88545159 .50645124 .04445093 .33815069 .98765035 .00594995 .0037 $\begin{array}{llllllll}4970.0022 & 4954.8945 & 4918.5499 & 4893.7494 & 4868.5819 & 4842.0868 & 4814.0918 & 4785.8827\end{array}$ $4761.3495 \quad 4729.9960 \quad 4694.6817 \quad 4685.9557 \quad 4714.2662 \quad 4787.3556 \quad 4872.9556 \quad 4914.3035$ 
Table 3.--Listing of data for 1971-77--Continued

Card

number

600.

601 .

602.

603.

604.

605.

606.

607.

608.

609.

610.

611.

612.

613.

614.

615 .

616.

617.

618.

619.

620 .

621 .

622 .

623.

624 .

625 .

626.

627.

628.

629.

630.

631.

632.

633.

634.

635.

636.

637.

638.

639.

640.

641.

642.

643.

644.

645 .

646.

647.

648.
Group III: Array data--Continued Starting head matrix, in feet--Continued
$4923.3998 \quad 4920.0902 \quad 4898.56914849 .12084788 .8874 \quad 4755.0000$ $\begin{array}{llllllll}0.0 & 6690.0000 & 6615.1210 & 6518.0209 & 6343.6387 & 6231.8817\end{array}$ $5828.5980 \quad 5729.2945 \quad 5664.5673 \quad 5617.0906 \quad 5582.6966 \quad 5562.4968$ $\begin{array}{lllllll}5483.6797 & 5456.2673 & 5431.6742 & 5398.6343 & 5359.7698 & 5326.2854\end{array}$ 5224.05375192 .97685163 .66125130 .00715100 .01645079 .8077 $\begin{array}{lllllll}4967.7351 & 4941.3048 & 4914.8496 & 4887.9383 & 4860.6410 & 4831.8735\end{array}$ $4740.6214 \quad 4719.9910 \quad 4695.9498 \quad 4693.7985 \quad 4746.2799 \quad 4824.6118$ $\begin{array}{llllll}4943.9196 & 4942.9287 & 4932.7018 & 4902.8220 & 4840.9798 & 4780.0000\end{array}$

$\begin{array}{lllllll}0.0 & 6690.0000 & 6611.1488 & 6521.2438 & 6366.9794 & 6231.3481\end{array}$ $5834.4002 \quad 5749.56275690 .7973 \quad 5640.17035607 .30525582 .8501$ $5503.76675468 .98405438 .1342 \quad 5404.31165368 .67335333 .0314$ 5224.43895190 .03375175 .02025173 .77955140 .50465091 .0435 $\begin{array}{llllllll}4965.1388 & 4940.0685 & 4914.8221 & 4884.9237 & 4855.0697 & 4825.0776\end{array}$ 4756.1156476 4963.8031 4747.6103 0.0
5864.1630 4960.6965

4746.3112 475

4752.1412 6690.0000660 4953.1912
6605.7713 16305777.03085720 .40075665 .5755 $5503.97725471 .56615436 .8478 \quad 5400.0815$ 5225.15105 5000.73474 4787.6616
5015.8067

0.0 5880.2155

5505.1261 5281.8605 5049.1783 4898.7812 5045.7457

0.0 5908.4562 5499.9935 5338.9599 5108.971850 5032.4683 5050.7817 0.0 5924.9488 5540.6927 5391.6628 5205.3694 5141.2533 5057.5252 0.0 5228.571 35 4805.1019 5003.4856
6700.0000 957.1027 219.51725 4806.1281 4881.6366 4896.7898 4825.0000 $6384.1373 \quad 6240.8671$ $5629.7570 \quad 5603.6027$ 5365.47515328 .2566 5173.03385111 .7050 4824.4318485 4940.1520491 913.9752 4985.1330 4960.5870 4895.1603 4909.7601 4856.4312 4949.7239 5815.7035 6591.9645
5753.9695 6496.2538 6376.3443 4870.0000 5469.5669 5276.7358 5031.0505 5432.4010 5690.1276 5646.8447 6249.1810 5620.5765 267.02225237 .16825177 .87475125 .0205 4906.5511 5016.5365 5017.9334 5006.7491 5037.4329 4918.0913 4943.7777 4969.6963 6640.0000 5012.3477 4983.0590 4932.6262 4956.5994 5016.2796 5852.3791578 6568.9941 6453.1959 6341.65776224 .3902 5470.001354 5787.70225720 .9018 5663.04765622 .8635 5326.4579 5439.97645424 .6164 5401.70465377 .4888 5095.55375078 .85135086 .9591 5200.11075192 .9514 5069.22825087 .2158 5010.56035008 .47205018 .1985 5038.90505063 .3094 5040.86305017 .7930 4986.3916 $4937.6404 \quad 4900.0000$ 6590.00006528 .18976403 .82936317 .32036209 .0215 $\begin{array}{lllllll}6584.9899 & 5799.9379 & 5734.8890 & 5660.0057 & 5614.9908\end{array}$ 5523.54915503 .63975476 .72245451 .00595431 .6500 5377.79125351 .08965309 .87595286 .57465268 .5475 5200.6165 5193.8619 5198.7833 5199.6727 5219.2915 5114.00535099 .56455093 .09635 5092.4583 5090.4378

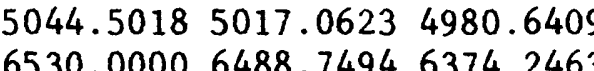
$4932.2246 \quad 4890.0000$
$6279.3614 \quad 6197.0046$ $\begin{array}{lllllll}5974.8833 & 5920.8186 & 5851.2907 & 5782.1367 & 5727.4580 & 5683.7032\end{array}$ $\begin{array}{lllllll}5586.1230 & 5568.2222 & 5550.9110 & 5522.2048 & 5494.2848 & 5475.3822\end{array}$ $\begin{array}{lllllll}5428.8864 & 5413.6799 & 5394.5649 & 5366.0307 & 5339.7939 & 5318.0628\end{array}$

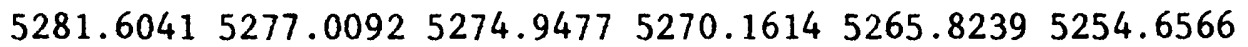
$5189.85175169 .61525149 .9430 \quad 5133.27915118 .7390 \quad 5106.5307$

\section{0}

6092.55835953 .2590 5546.35045519 .9837 5294.21795258 .7324 $5031.3659 \quad 4991.6495$ $4796.1053 \quad 4764.5864$ $4905.5175 \quad 4936.3338$ 0.0

6106.55155966 .5980 5561.66755538 .2788 $5296.7040 \quad 5260.3266$ $5037.7861 \quad 4996.2884$ $4795: 1935 \quad 4772.0877$ 4950.52184960 .9115 0.0

6107.57025987 .8633 $5576.2570 \quad 5542.2542$ $5297.2219 \quad 5269.3754$ 5061.01195024 .9203 4810.17274791 .5034 4998.28905015 .8560 0.0

$6119.9570 \quad 5989.3074$ 5582.72005541 .0904 5300.05685294 .9619 5085.11225069 .8947 $4925.2795 \quad 4903.5126$ 5049.41475051 .3437 0.0

6105.00695995 .7799 5583.10955541 .8344 $5357.2948 \quad 5346.1069$ 5163.53365136 .1888 5089.60795070 .4997 5066.41625059 .2305 0.0

6106.07386005 .6302 5584.96465549 .9725 5414.20315400 .5537 $5244.5320 \quad 5218.5888$ 5208.71565180 .6254 5081.33115069 .2548 0.0

6117.71196030 .0149 5650.27235617 .2694 $5458.6150 \quad 5442.5664$ 5299.79185283 .3669 5237.59575214 .1092 5093.96175080 .8676 
Table 3.--Listing of data for 1971-77--Continued

Card number

649.

650.

651 .

652.

653.

654.

655 .

656.

657 .

658 .

659.

660.

661 .

662.

663.

664.

665.

666 .

667.

668.

669.

670.

671 .

672.

673.

674.

675.

676.

677.

678.

679 .

680 .

681 .

682 .

683.

684.

685.

686.

687.

688 .

689.

690.

691.

692.

693.

694.

695.

696.

697.
Group III: Array data--Continued

Starting head matrix, in feet--Continued

$$
\begin{aligned}
& 5066.0446 \\
& 0.0
\end{aligned}
$$

6043.2898

5642.4442

5452.0935

5307.9183

5198.4996

5073.1342

0.0

6114.5208605

5688.4544

5471.6395

15

5326.0371

5314.6833

5071.8585

5183.6697

0.0

0.0

0.0

5015.3442
6442.1193

4966.5702

4924.0327

6259.9926

5898.9316

5353.10726

5787.5350

5591.8065

5563.4506

5531.8882

5414.4581
5290.8582

5391.9379

5370.0932

5156.1825

5282.3722
5137.7252

5272.6341

5008.1243

4957.0844

6400.01046345 .0170

5122.1783
4918.3972

5971.0429

5885.1096

6299.0688

5842.0806

4870.0000

6190.0011

5745.7776

5510.3494
5350.7616

5259.4503

5109.9473

4860.0000

6245.6307

5804.4644

5626.1761

5595.7559

5564.4393

5303.5145

5292

5390.3226

5537.2839

5371.4747

5160.7478

5139.

5279.6186

5265.0248

5002.0652

4951.3304

5121.7589

6430.0000

6410.2005

6341.07

5110.8513

6172.56586116 .7068

6037.1729

5942.3528

5876.3771

5726.77025687 .7228

5651.3012

5618.6468

5588.1750

5468.7927

5448.1781

5427.7750

5407.8891

5338.57965324 .9594

5312.1218

5298.7780

5284.8136

5123.7610

5213.0989
5060.4798

5191.4776

5168.5621

5146.1713

4912.2194

4996.0962
6498.1077

4951.2120

6403.8388

6479.588

6217.1713

6530.0000

6067.5778

65637.9151

5909.09215864 .7224

5749.8833

5709.3161

5673.0726

5443.1772

5605.282

5576.2222

5505.64415484 .3845

5463.5364

5302.1411

5423.387

5404.2903

5348.9352

5333.9801

5176.12945153 .3358

5287.9431

5272.3612

5045.7550

5024.7890

4996.6926

4961.4562

5127.7197

5103.4061

0.0

6570.0000

6553.2384

6525.3716

4915.7931
6454.5784

4870.0000

6246.1021

5766.9215

6180.6565

6093.9756

5994.3759

5921.0925

6412.3859

5692.8661

5655.8402

5621.6011

5880.6729

5520.2139

5730.3337

5477.1835

5456.8131

5437.0170

5592.8912

5358.2475

5341.2672

5325.4928

5308.1541

5291.3303

5418.2583

5215.0200

5043.6123

0.0

5197.6196

5182.2689

5160.2450

5133.9047

5273.9928

4918.03964875 .0000

6595.0000

6630.7148

4963.1793

$6478.3200 \quad 6430.1308$

6245.7993

5780.3379

6182.3976

6088.0101

6545.8555

5934.71625884 .8874

5536.5012

5370.14355350 .38655

5042.6243

5023.8437

749

5183.7290

4997.7225

6625.00006649 .1311

6220.5850

5790.7748

6158.6976

6092.2365

5

5671.9278

5638.1128

5610.2411

5556.2923

5378.4333535

5219.04345203 .3330

0.0

6125.09026097 .2939

5709.23295678 .8600

5490.07055471 .5110

5334.84155319 .7903

5242.51195221 .3051

5098.01425087 .4963 0.0

6189.17976162 .7769

5766.91345727 .5848

5513.65955492 .1882

5354.52465339 .3150

5248.27295228 .6901

5097.34145085 .1952

0.0

6251.22056216 .1559

5808.90305769 .6503

5533.16695510 .9092

5371.02875354 .0313

5253.22095234 .0805

5089.12115079 .3336 0.0

6317.76416267 .9056

5828.93725791 .0125

5550.15995527 .4948

5385.41195366 .0225

5256.73645237 .6342

5085.18645068 .2771 0.0

6362.92286298 .6635

5840.74035801 .5996

5566.62515542 .4799

5399.01985378 .6952

5255.83545235 .8444

5083.66115062 .3378 0.0

6356.77376285 .1111

5849.81045815 .5204

5584.49535559 .8874 
Table 3.--Listing of data for 1971-77--Continued

Card

number

698.

699.

700 .

701 .

702 .

703.

704.

705.

706.

707.

708.

709 .

710 .

711.

712 .

713.

714.

715 .

716.

717.

718 .

719.

720 .

721.

722 .

723.

724.

725 .

726.

727.

728.

729 .

730 .

731.

732.

733.

734.

735.

736.

737.

738.

739 .

740 .

741 .

742.

743.

744.

745 .

746 .
Group III: Array data--Continued Starting head matrix, in feet--Continued

\begin{abstract}
$5043.502 .95025 .47875000 .1176 \quad 4965.4520 \quad 4918.5374 \quad 4865.0000$
$0.0 \quad 6675.45996660 .04256565 .0636 \quad 6481.29746407 .7091$

$\begin{array}{lllllll}6206.4811 & 6150.1235 & 6093.8520 & 6018.8750 & 5944.9466 & 5898.1481\end{array}$

$\begin{array}{lllllll}5792.2898 & 5757.7495 & 5727.7530 & 5700.8902 & 5672.2331 & 5641.3906\end{array}$

$\begin{array}{lllllll}5569.7226 & 5545.9769 & 5519.1056 & 5495.1437 & 5474.3084 & 5453.4706\end{array}$

$\begin{array}{llllll}5389.3268 & 5367.7016 & 5347.2243 & 5326.8800 & 5305.5420 & 5282.4946\end{array}$

5219.4706

5200.7074

5180.3846

5158.37395134 .7252

5107.1308

5045.0953

0.0

6707.3424

5003.0037

4968.1029

4918.1361

4860.0000

6194.7486

6141.1147

6670.0068

6575.19666475 .7336

6407.2815

5790.0434

5757.7366

5400.8762

5552.7747
5378.9041

5731.8820

6009.23985946 .1343

5900.0907

5217.2644

5050.7214

5196.6255

5528.5906

5504.45705482 .6639

5651.0802

5030.2190

5175.8849

5336.5019531

313.1899528

5462.4118
5288.5257

5004.2081

4968.6876

918.6810 4860.0000

$\begin{array}{ll}0.0 & \\ 6329.5484 & 6261.2496\end{array}$

5861.96705827 .1415

5614.54915592 .2052

5435.37045413 .1884

5260.11595239 .2876

5080.09605061 .1059

0.0

$6332.0228 \quad 6256.3386$

$\begin{array}{lll}5861.7140 & 5827.1522\end{array}$

5623.18845597 .3712

5444.64915424 .4801

5262.50455238 .5637

5089.61995069 .8808

0.0
\end{abstract}

0.0

6192.4042

6723.8094

6132.9348

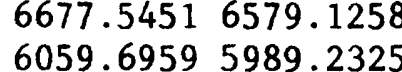

5790.0583

5758.8486

5584.4738

5562.5145

5734.0512

5708.7983

6477.7367

6410.4980

5409.6712

5387.9317

5537.5771

5514.2546

590.5823

5906.6640

5212.0611

5190.1388

5366.59965344 .6351

5492.4537

5656.6547

5065.0861

5043.0573

5011.9523

5150.7183

5320.7468

5

0.0

6735.5169

6681.6042

6577.9631

4917.5022

5116.7384

4865.0000

6425.4881

6188.1040

6134.9546

6069.60175998 .338

6485.9067

5896.7766

5790.1916

5761.0626

5736.24035712 .3685

5687.7215

5662.4706

$5591.6760 \quad 5570.2517$

5547.33475524 .738

5503.11375482 .1025

5417.1953

5395.4568

5373.7960

5350.6855

5325.6547

5297.7881

5202.9754
5072.9212

5180.7353

5161.0733

5143.9120

$\begin{array}{lll}4967.5099 & 4916.4193 & 4865.0000 \\ 6575.7169 & 6501.9244 & 6431.9927\end{array}$

6735.0098

6666.6147

6193.1529

6145.7481

6084.6424

6004.9611

5937.0732

5888.8365

5787.5477

5761.9492

5738.8235

5715.4383

551

5691.46125666 .9801

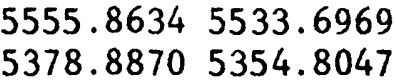

5512.31145491 .5818

5600.17715578 .2839

5328.3744529

5297.2974

5131.5006

5116.6819

5104.9217

5070.8361

5168.9027

5149.0399

4964.9281

4914.04444860 .0000

6509.91916447 .8623

6659.07546576 .6003
6090.05876010 .9223

5936.1271

5888.7266

6199.2719

6700.0000

5694.6109

5669.2137

5606.0506

5761.6938

5740.35045718 .0105

5518.0713

5499.3649

5328.26935294 .1964

5176.7927

5404.2257

5381.60525356 .4702

5101.9205

5091.7642

4860.0000

6511.32456459 .2680

0.0

5039.2681

5132.64085115 .4145

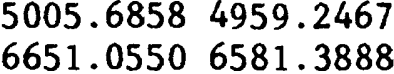

6201.8745

6660.0000

6080.64685996 .0949

5923.7656

5875.8000

5782.4999

5762.2229

5741.56905720 .2951

5698.3590

5675.6675

5501.3986

5430.17375408 .1693

556.26995542 .94095521 .2310

5156.3502

5408.1693
5134.3031

5111.8767

5355.25225323 .5365

5285.9946

$6327.6280 \quad 6250.3642$

$5864.5230 \quad 5827.2298$

5628.42595603 .6246

5451.82065431 .5830

5264.94135235 .7099

5099.02735082 .1656

0.0

6341.26316249 .2243

5859.71765824 .4249

5637.16985613 .0292

5460.89805439 .2973

5265.50415231 .7440

5099.16145086 .1685

0.0

6348.70756258 .1888

5851.67695820 .7825

5643.43735621 .5463

5469.21155446 .3267

5260.44155222 .8851

5094.31135082 .9789

0.0

6354.43546254 .0028

5849.30355812 .3304

5645.94515626 .6426

5477.04465451 .4145

$5252.8910 \quad 5211.1574$

5083.67455076 .1862 0.0

6378.04736269 .8471

5839.53995808 .8060

$5653.1970 \quad 5632.8755$

5478.83645453 .3251

$5240.6958 \quad 5192.7658$

5065.23625058 .7369 
Table 3.--Listing of data for 1971-77--Continued

Card

number

747.

748 .

749 .

750 .

751 .

752 .

753.

754 .

755 .

756 .

757.

758 .

759 .

760 .

761 .

762 .

763.

764.

765 .

766.

767.

768 .

769.

770 .

771 .

772.

773.

774 .

775 .

776 .

777 .

778 .

779 .

780 .

781 .

782 .

783 .

784 .

785 .

786 .

787 .

788 .

789 .

790 .

791 .

792.

793.

794.

795.
Group III: Array data--Continued

Starting head matrix, in feet--Continued
5042.4201

$0.0 \quad 6630.0000$

6206.3807

$5783.2740 \quad 5761.8385$

5619.66845597 .0025

5426.92315404 .9848

5142.22535119 .564750

5021.61905002 .5566

0.0

0.0

6211.01076133 .7290

5783.03215762 .2254

$5622.21825598 .6428 \quad 55$

5421.1322539

5146.1935

4998.7055

5397.1105

5119.1621

0.0

$$
0.0
$$

8495

4991.5241

6628.9907

6069.9527

5742.10935721 .9

5570.50865545 .6435

5380.1286

5349.7791

5095.24015079 .7208

$4972.7776 \quad 4934.5111$

6615.00006569 .3801

4904.62544850 .0000

6486.4020

5910.9437

5867.3637

5701.5241

5680.4956

5522.5263

5500.8622

6207.97176129 .3753

5782.28815761 .8392

6048.0282

5964.7292

5068.1712

4898.7058

$6517: 6260$

5055.6317

4840.0000

6487.0882

5741.8056

5722.4858

5903.5732

5863.7737

5684.3793

5372.3770

5544.88785520 .6765

5496.3321

5093.1146507

5344.5624

5309.3014

5266.8484

5041.6238

40.35184922 .67804897 .15714860 .0000

$4922.6780 \quad 4897.1571$
6539.1421
6479.5632

4860.0000

6030.15785963 .40645904 .20985865 .1451

5740.1252572

5721.12165706 .5667

5688.2325

5620.65465596 .6788

5416.21645391 .8187

5571.0790

5544.5072

5518.0576

5492.2981

5154.44465129 .6677

5367.9086

5341.2101

5306.9896

5267.1833

4983.6140

0.0

0.0

4949.7528

5073.7847

5049.2501

5027.8311

0.0

6485.00006414 .3213

6346.8027

05

5782.9806

$5617.94465595 .5321 \quad 5570.4985 \quad 554$

5721.50595705 .4098

5866.6453

5687.1441

5411.40135386 .61905362 .988053

5165.80395140 .95915112 .265150

$4985.2536 \quad 4970.3324 \quad 4950.2780$

$$
0.0
$$

0.0

0.0

4932.8682

$6180.0400 \quad 6118.9504 \quad 6047.151859$

5786.12265763 .56445742 .862057

$\begin{array}{llll}5621.0113 & 5596.6615 & 5570.6163 & 554 \\ 5406.9461 & 5382.2136 & 5360.6232 & 533\end{array}$

5176.02855147 .53125118 .08195

$4994.3642 \quad 4991.0568 \quad 4988.9975$

0.0

0.0

0.0

6480.0000

$\begin{array}{lllll}6155.0041 & 6100.4873 & 6047.1728 & 5964.0180 \\ 5789.8937 & 5766.9778 & 5747.6761 & 5728.0423\end{array}$

5624.96025599 .88175569 .26265536 .5718

5404.49155379 .86395358 .44295329 .9365

5181.88345152 .80275122 .27675091 .3838

5056.07975051 .76195048 .9575

$$
0.0
$$

0.0

0.0

5033.13035019 .7496

6515.00006457 .3896

5488.3897

5265.9783

504.5743
5051.5166
495.5 .8649

5026.4361

4930.0000

6400.00876325 .0210

5866.0440

0.0

6384.82036258 .1163

5836.96225809 .3720

$5659.3508 \quad 5638.9122$

5476.81045450 .8099

5227.60445177 .9434

5043.01225034 .5365

0.0

$6393.7734 \quad 6284.0569$

5835.57665807 .6846

5663.47275642 .4739

5470.77135445 .3743

5221.84815179 .3740

5023.82165011 .5368 0.0

6355.97176272 .6431

5833.35685805 .1126

5666.79195644 .0889

5466.66165441 .2045

5224.95015184 .8366

$5006.5043 \quad 4995.9925$

$$
0.0
$$

6299.01936253 .0788

$6144.99526087 .87046035 .73845960 .8808 \quad 5901.7912587$

$5798.44835775 .35475754 .03635732 .8588 \quad 5710.68965686 .5616 \quad 5663.87875644 .7743$

$5625.34395599 .8300 \quad 5567.78695533 .13835503 .0513 \quad 5480.1055 \quad 5455.88345430 .4039$

$\begin{array}{llllllllll}5403.2086 & 5378.6042 & 5356.8760 & 5327.2628 & 5293.4451 & 5266.7837 & 5241.7996 & 5213.7378\end{array}$

$5185.76885156 .6565 \quad 5124.4085 \quad 5088.2934 \quad 5064.2720 \quad 5057.02835051 .65485095 .5589$ 
Table 3.--Listing of data for 1971-77--Continued

Card

number

796.

797.

798.

799.

800 .

801 .

802 .

803.

804.

805 .

806.

807 .

808 .

809 .

810 .

811.

812.

813.

814.

815 .

816 .

817.

818.

819 .

820 .

821 .

822 .

823.

824.

825 .

826.

827.

828.

829.

830 .

831.

832.

833.

834.

835.

836.

837.

838.

839.

840 .

841.

842 .

843.

844.
Group III: Array data--Continued

Starting head matrix, in feet--Continued $\begin{array}{ccc}5122.4047 & 5097.5108 \\ 0.0 & 0.0 & 601\end{array}$

$6153.3766 \quad 6069.9490$

$5805.2708 \quad 5783.1762$

5622.41085596 .4315

5403.27295378 .8073

5194.28455163 .8509

5131.68765121 .3996

0.0

0.0

6164.28056057 .8891

5810.17535790 .1220

5620.87945595 .8146

5405.10625381 .1128

5202.18495176 .9018

5149.15285143 .5496

0.0

0.0

6190.00796085 .9659

5810.46985788 .3311

5620.02745599 .8923

5405.47245384 .3521

5206.51875185 .2430

5160.44315155 .0812

$$
0.0
$$

0.0

6215.89646109 .9699

5808.93245784 .7175

5616.33115591 .0591

5403.37575382 .7073

5209.15375190 .7083

5166.0363

0.0

5161.3012
7140.0000

6223.4856

5805.4851

5609.8493

5393.9613

5211.2239

5170.8249

0.0

6206.6285

5801.6682

5599.2408

5380.2103

5212.6350

5175.9244

0.0

6176.4986

5798.4330

5583.5707

5363.9658

5215.7466 6117.8257

5582.0032

5194.0485

7110.0000

6109.1536

(

195.8821

7080.0000
5084.28715063 .04795034 .75835000 .0000

$6655.00006589 .8128 \quad 6490.9521$

6016.65295963 .79895913 .4309

5760.8769573

5739.0323

5715.2471

5875.0835

$5566.6876 \quad 5533.2628 \quad 5502.7026 \quad 5478.8294$

$\begin{array}{lllll}5356.4950 & 5327.7485 & 5295.2299 & 5268.7247\end{array}$

5127.86635091 .76615069 .8997

5104.1847

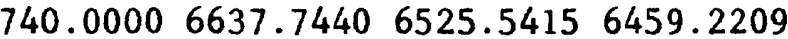

6010.02855969 .98525920 .0617

5765.067

5567.84885538 .9646

5355.15305324 .4303

5142.82615101 .8160

5127.30385103 .1228

6850.00006671 .3816

6020.9365

5979.904459

5764.692757

5569.91915544 .519255

5356.95695322 .4680

5155.36215115 .562050

5142.24615119 .9481

6890.00006704 .0515

5719.9770

5890.1174

6034.28855985 .9942593

5295.2637

5477.2085

5760.6347

5737.5988

5078.9225

5272.1239

5071.4613

5562.67415533 .5658

5070.6441

5040.0000

6548.03106477 .4853

5884.9392

5716.60875692 .3707

5354.27215325 .2400

5510.4474

5479.0098

293.7877

5274.5187

5091.4510

5087.4009

5060.0000

6486.4488

6553.4731

5878.9559

5713.57355689 .6178

5163.5607

5127.5924

5509.6229

5474.7140

5298.03845274 .5972

5149.84775130 .20785101 .07825065 .0000

$6884.8206 \quad 6708.34986571 .8303$

6496.9481

6042.6312

$5989.9090 \quad 5928.2174$

5874.3432

5548.57735

5733.65045709 .6387

5685.3737

5349.1069

5512.2300

5480.5041

5447.0429

5156.7291513

5136.9995

5297.9147

5273.5086

5139.8110511

0503

5127.5258

5070.0000

678710.50236582 .4056

6505.0148

5867.5445

048.40135994 .886

5922.6716

73567

679.0258

5521.76805476 .56865438 .2948

5294.0993

5

5171.12365146 .74155134 .6802

5

6889.7666 .6697 .2159

658

581.1487

\section{8}

6104.05416045 .70015986 .33665911 .1803

5865

5748.51535723 .65975697 .8002
5671.1225

$\begin{array}{llllll}5494.7929 & 5443.29375405 .7568 & 5386.9885\end{array}$

5328.59335309 .38475288 .07475268 .2298

$5175.74515155 .9300 \quad 5148.30645155 .0325$
0.0

6360.65666269 .6055

5852.42645828 .0232

5667.54745645 .0381

5454.65515429 .6789

5245.30465220 .2773

5073.10805108 .2865 0.0

6384.49616289 .5449

5865.05665835 .1543

5670.16715645 .8012

5453.61245429 .4274

5249.10575226 .3917

5094.74985129 .9341 0.0

6399.05016301 .1095

5852.47235832 .6644

5669.98235645 .0011

$5452.5110 \quad 5428.1535$

5251.97725228 .2589

0.0

6402.80946319 .1265

5848.00165830 .1174

5665.95505641 .5165

$5443.3898 \quad 5420.3325$

5249.63445227 .1465

5145.35865165 .7530 0.0

6415.03656325 .6318

5845.24315826 .8022

5661.83085637 .1506

5423.98925406 .3627

5248.78135227 .8034

5158.24665173 .5683 0.0

6417.33216315 .2998

5840.97975823 .2755

5653.72625627 .3498

5400.63855388 .1177

5248.54195229 .8951

5165.16775178 .8904 0.0

6401.85856267 .5749

5839.37015820 .4632

$5644.1588 \quad 5614.6974$

$5379.2498 \quad 5372.5742$

5251.34225232 .6917

5172.58285185 .4172 
Table 3.--Iisting of data for 1971-77--Continued

Card

number

845 .

846.

847.

848 .

849.

850 .

851.

852 .

853.

854.

855 .

856.

857.

858 .

859 .

860 .

861 .

862 .

863.

864.

865 .

866 .

867.

868 .

869 .

870 .

871 .

872.

873.

874.

875 .

876 .

877.

878 .

879 .

880 .

881 .

882 .

883 .

884 .

885 .

886 .

887 .

888 .

889 .

890 .

891 .

892.

893.
Group III: Array data--Continued

Starting head matrix, in feet--Continued

\begin{abstract}
$5182.78145177 .6655 \quad 5169.33875155 .54065133 .31165090 .0000$
$\begin{array}{lllllll}0.0 & 7100.0000 & 6854.1519 & 6680.9184 & 6566.2253 & 6492.6317\end{array}$

0.0

$\begin{array}{llllllllll}5795.9287 & 5769.8003 & 5743.5020 & 5717.1918 & 5689.8911 & 5661.3450 & 5631.7238 & 5597.8468\end{array}$

$5563.89835525 .92265475 .2081 \quad 5426.19275392 .2277 \quad 5376.4846 \quad 5366.9927 \quad 5358.4279$

$\begin{array}{llllllllll}5347.5907 & 5332.6795 & 5318.1521 & 5302.5579 & 5285.2660 & 5269.5842 & 5254.8863 & 5237.3762\end{array}$

$\begin{array}{llllllll}5218.4939 & 5200.2706 & 5179.1181 & 5165.2381 & 5161.2828 & 5164.3642 & 5178.5326 & 5216.2236\end{array}$

$5191.09725185 .42715176 .64995162 .89895142 .3912 \quad 5100.0000 \quad 0.0$
\end{abstract}

$\begin{array}{lllllllll}0.0 & 7050.0000 & 6807.2992 & 6643.8568 & 6535.2881 & 6427.3694 & 6265.4101 & 6165.7838\end{array}$

$\begin{array}{llllllllll}6095.2219 & 6055.5826 & 6028.7419 & 5969.7660 & 5903.7390 & 5865.2246 & 5841.7843 & 5819.5297\end{array}$

$\begin{array}{llllllllll}5792.9543 & 5763.7215 & 5736.4538 & 5709.3386 & 5680.9963 & 5650.8272 & 5615.8440 & 5576.0537\end{array}$

$5539.76255501 .78115458 .72635414 .01525381 .6657 \quad 5368.7143 \quad 5357.7440 \quad 5346.7660$

$\begin{array}{llllllll}5335.0012 & 5321.8013 & 5309.5194 & 5294.8279 & 5280.2194 & 5268.6630 & 5254.1473 & 5236.9186\end{array}$

$5219.37195201 .39095183 .04395172 .1650 \quad 5170,01245171.52625185 .3055 \quad 5233.5771$

$5205.00915194 .65525183 .90235170 .19905150 .62415120 .0000 \quad 0.0$

$0.0 \quad 7000.00006750 .10096594 .3986 \quad 6470.7503 \quad 6348.99146205 .05906106 .1397$

$6030.80116007 .50985994 .8390 \quad 5948.8856 \quad 5901.9556 \quad 5868.36645847 .62445821 .0393$

$\begin{array}{llllllllll}5789.8938 & 5758.5381 & 5729.9569 & 5701.4190 & 5670.3277 & 5632.2286 & 5583.5030 & 5542.3381\end{array}$

$\begin{array}{llllllllll}5507.4483 & 5473.6154 & 5443.3121 & 5408.4859 & 5378.2000 & 5359.7166 & 5346.9139 & 5335.1480\end{array}$

$\begin{array}{llllllll}5321.5430 & 5308.1400 & 5295.8073 & 5282.2126 & 5272.1148 & 5262.5266 & 5248.5732 & 5234.4715\end{array}$

$\begin{array}{llllllll}5218.6700 & 5200.8556 & 5185.1999 & 5177.7587 & 5176.2680 & 5176.8327 & 5190.9879 & 5241.3965\end{array}$

$\begin{array}{llllllll}5214.3990 & 5203.7470 & 5190.3355 & 5177.0482 & 5157.4629 & 5125.0000 & 0.0\end{array}$

$\begin{array}{llllllll}0.0 & 6900.0000 & 6707.9730 & 6536.5317 & 6377.4678 & 6266.9525 & 6147.2096 & 6038.8893\end{array}$

$\begin{array}{lllllllll}5972.0217 & 5956.9697 & 5956.3445 & 5931.0993 & 5894.1088 & 5871.8962 & 5851.9928 & 5827.5363\end{array}$

$5789.50825749 .0895 \quad 5719.66845685 .28225643 .5018 \quad 5593.6321 \quad 5544.96715512 .5528$

$\begin{array}{lllllllll}5477.8076 & 5448.0074 & 5425.8062 & 5397.1689 & 5371.3296 & 5349.6198 & 5334.6490 & 5320.5868\end{array}$

$\begin{array}{lllllllll}5305.2262 & 5290.1355 & 5279.6505 & 5268.8232 & 5261.2339 & 5251.5228 & 5241.1181 & 5230.6463\end{array}$

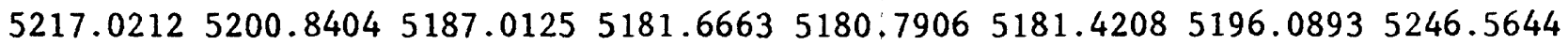
$\begin{array}{lllllll}5220.8430 & 5210.3647 & 5194.2817 & 5183.5503 & 5165.4956 & 5140.0000 & 0.0\end{array}$

$\begin{array}{llllllll}0.0 & 6870.0000 & 6670.1010 & 6475.9019 & 6323.3127 & 6232.4865 & 6110.9736 & 5984.9915\end{array}$ $5921.92525912 .7045 \quad 5913.94895910 .5195 \quad 5892.1422 \quad 5873.63695850 .68205820 .6423$ $\begin{array}{llllllll}5781.0057 & 5738.0095 & 5698.2448 & 5657.1612 & 5611.3263 & 5564.9729 & 5521.1797 & 5485.3889\end{array}$ $5446.5588 \quad 5423.6764 \quad 5401.7561 \quad 5377.4440 \quad 5355.0446 \quad 5333.03645315 .7849 \quad 5298.5754$ $5286.94075275 .24465266 .82715254 .4541 \quad 5243.9112 \quad 5233.03395228 .6371 \quad 5222.4514$ $5214.76185203 .09545190 .45495184 .1253 \quad 5183.8438 \quad 5185.90665201 .4856 \quad 5251.8408$ $\begin{array}{lllllll}5226.0472 & 5214.5041 & 5196.2949 & 5187.0708 & 5171.5221 & 5150.0000 & 0.0\end{array}$

$\begin{array}{llllllll}0.0 & 6800.0000 & 6617.5166 & 6416.8610 & 6276.0210 & 6176.1351 & 6050.8759 & 5928.9516\end{array}$ $\begin{array}{llllllll}5850.1975 & 5854.5782 & 5870.8755 & 5882.6282 & 5879.7869 & 5864.5523 & 5839.3544 & 5802.7776\end{array}$ $5755.34765709 .6705 \quad 5668.72555623 .96815578 .81065533 .9653 \quad 5490.52955454 .2014$ $\begin{array}{lllllllllll}5418.6747 & 5400.0240 & 5376.0004 & 5354.6793 & 5334.1382 & 5310.1609 & 5288.9571 & 5272.6929\end{array}$

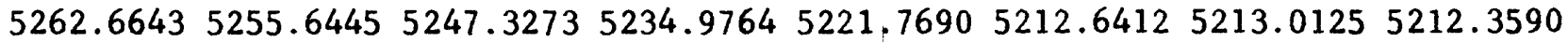
$5213.76525210 .33125200 .57435192 .06385191 .16125193 .6365 \quad 5209.34685258 .8544$ $5230.59535217 .47225197 .66495188 .7290 \quad 5174.3417 \quad 5160.0000 \quad 0.0$

$0.0 \quad 6700.00006543 .05246363 .7265 \quad 6213.68236102 .5855 \quad 5984.08615866 .0045$ $5794.25165794 .7723 \quad 5820.40065853 .0193 \quad 5860.2275 \quad 5847.25225818 .94295772 .0504$

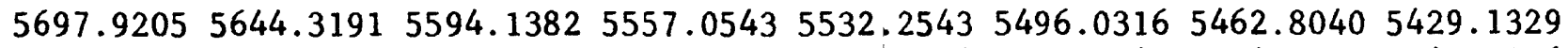

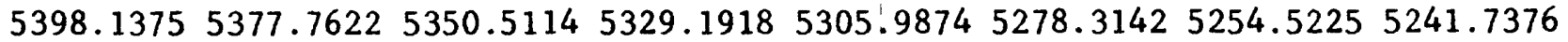

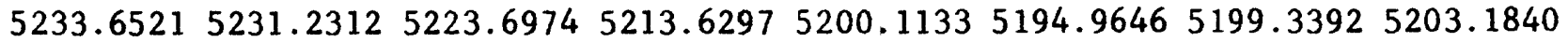
5211.03735215 .44145214 .49655208 .20585207 .14465209 .53905253 .09525272 .4995 
Table 3.--Listing of data for 1971-77--Continued

Card

number
Group III: Array data--Continued

Starting head matrix, in feet--Continued

894.

895.

896.

897.

898.

899 .

900 .

901.

902.

903.

904.

905.

906.

907.

908.

909.

910.

911.

912.

913.

914.

915.

916.

917.

918.

919.

920.

921.

922.

923.

924.

925.

926.

927.

928.

929.

$$
\begin{gathered}
5235.2048 \\
0.0
\end{gathered}
$$

5220.668
6630.0000

5199.1115518

5189.6039517

$\begin{array}{ll}5175.0526 & 5150.0000 \\ 6139.4947 & 6027.0745\end{array}$

0.0

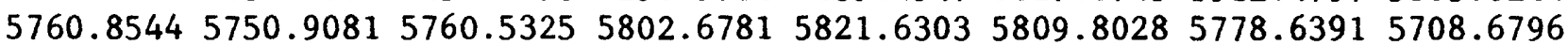

$5611.13995561 .90385509 .93075485 .4446 \quad 5470.7477 \quad 5445.61315421 .83845392 .1137$

$5369.31625345 .40575319 .16175302 .6080 \quad 5276.0378 \quad 5246.40025223 .3996 \quad 5213.7343$

$\begin{array}{llllllllll}5208.1343 & 5201.9558 & 5194.3767 & 5188.2420 & 5177.4577 & 5170.0540 & 5175.1129 & 5188.7491\end{array}$

5202.01805214 .40885219 .93035219 .54245221 .64455226 .55685251 .66605258 .4568

5244.856252

0.0

5225.2003

5200.7298

5189.78135175 .1148

5150.0000

0.0

5731.336357

6550.0000

$6380.9578 \quad 6224.5227$

6074.1922

5972.10475

5862.83945765 .8495

5329.02235305 .98885291 .40525275 .23365246 .65685220 .31845200 .64115190 .2483

$5183.43255174 .02875166 .04375157 .86015150 .6385 \quad 5140.23225133 .46375144 .8508$

$5171.99145196 .88925211 .29745218 .7388 \quad 5226.55595234 .44575255 .17845260 .4656$

$5252.14285237 .79625209 .83065188 .61275174 .53215150 .0000 \quad 0.0$

$\begin{array}{lllllllll}0.0 & 6470.0000 & 6296.0120 & 6139.3125 & 5994.8699 & 5904.6824 & 5803.3893 & 5722.3647\end{array}$

$\begin{array}{llllllll}5692.9923 & 5682.8328 & 5665.4815 & 5653.8672 & 5634.4014 & 5605.9477 & 5563.7138 & 5512.0535\end{array}$

$\begin{array}{llllllllll}5470.0503 & 5436.1403 & 5406.8739 & 5384.3687 & 5360.6420 & 5341.3487 & 5326.4121 & 5306.5525\end{array}$

$5285.45885267 .6776 \quad 5254.8165 \quad 5234.9714 \quad 5210.5921 \quad 5192.02285175 .6190 \quad 5164.1017$

5153.55385143 .43355134 .39055124 .90725118 .16775110 .01745101 .04615097 .4648

$\begin{array}{llllllll}5120.6647 & 5150.9321 & 5175.8330 & 5199.2580 & 5222.1659 & 5243.6468 & 5259.7666 & 5262.0931\end{array}$

5252.8685524

5244.70225216 .56095188 .41145173 .21095150 .0000

0.0

0.0

6350.0000

6200.0000

6030.00005900 .0000

5820.0000

5750.00005690 .0000

$5650.0000 \quad 5630.0000 \quad 5610.0000 \quad 5590.0000 \quad 5570.0000 \quad 5550.0000 \quad 5510.0000 \quad 5460.0000$

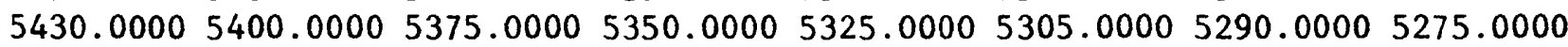

5260.00005235 .00005220 .00005205 .00005185 .00005170 .00005145 .00005130 .0000

5115.00005105 .00005095 .00005085 .00005080 .00005075 .00005070 .00005070 .0000

$5070.00005065 .00005090 .00005140 .0000 \quad 5190.0000 \quad 5245.0000 \quad 5250.0000 \quad 5250.0000$

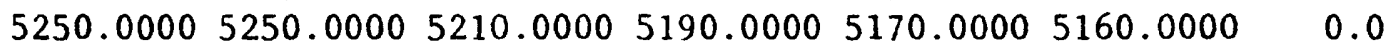

$\begin{array}{llllllll}0.0 & 0.0 & 0.0 & 0.0 & 0.0 & 0.0 & 0.0 & 0.0 \\ 0.0 & 0.0 & 0.0 & 0.0 & 0.0 & 0.0 & 0.0 & 0.0 \\ 0.0 & 0.0 & 0.0 & 0.0 & 0.0 & 0.0 & 0.0 & 0.0 \\ 0.0 & 0.0 & 0.0 & 0.0 & 0.0 & 0.0 & 0.0 & 0.0 \\ 0.0 & 0.0 & 0.0 & 0.0 & 0.0 & 0.0 & 0.0 & 0.0 \\ 0.0 & 0.0 & 0.0 & 0.0 & 0.0 & 0.0 & 0.0 & 0.0 \\ 0.0 & 0.0 & 0.0 & 0.0 & 0.0 & 0.0 & 0.0 & \end{array}$

Group III: Array data--Continued

Storage coefficient, dimensionless

(First card is parameter card)

930 .

931.

932.

933.

934.

935.

936.

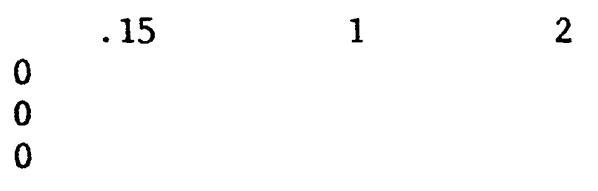

2 
Table 3.--Listing of data for 1971-77--Continued

Card

number
Group III: Array data--Continued

Storage coefficient, dimensionless--Continued
937.

938.

939 .

940.

941.

942.

943.

944.

945.

946.

947.

948.

949.

950.

951.

952.

953.

954.

955.

956.

957.

958.

959.

960.

961.

962.

963.

964.

965.

966.

967.

968.

969.

970.

971.

972.

973.

974.

975.

976.

977.

978.

979.

980.

981.

982.

983.

984.

985 . $\begin{array}{llllllllllllllllll}1 & 1 & 1 & 1 & 1 & 1 & 1 & 1 & 1 & 1 & 1 & 1 & 1 & 1 & 1 & 1\end{array}$

$\begin{array}{llll}1 & 1 & 1 & 1\end{array}$

$\begin{array}{rlllllllrrrrrrrrrrrr}1 & 1 & 1 & 1 & 1 & 1 & 1 & 1 & 1 & 1 & 1 & 1 & 1 & 1 & 1 & 1 & -1 & -1 & 1 & 1\end{array}$ $\begin{array}{llllllllllllllllllll}1 & 1 & 1 & 1 & 1 & 1 & 1 & 1 & 1 & 1 & 1 & 1 & 1 & 1 & 1 & 1 & 1 & 1 & 1 & 1\end{array}$ $\begin{array}{llllllllllllll}1 & 1 & 1 & 1 & 1 & 1 & 1 & 1 & 1 & 1 & 1 & 1 & 1 & -1\end{array}$ $\begin{array}{rllllllllllllllllll}-1 & 1 & 1 & 1 & 1 & 1 & 1 & 1 & 1 & 1 & 1 & 1 & 1 & 1 & 1 & 1 & 1 & 1 & 1\end{array}$ $\begin{array}{llllllllllllllllllll}1 & 1 & 1 & 1 & 1 & 1 & 1 & 1 & 1 & 1 & 1 & 1 & 1 & 1 & 1 & 1 & 1 & 1 & 1 & 1\end{array}$ $\begin{array}{llllllllllllll}1 & 1 & 1 & 1 & 1 & 1 & 1 & 1 & 1 & 1 & 1 & 1 & 1 & -1\end{array}$ $\begin{array}{llllllllllllllllllll}-1 & 1 & 1 & 1 & 1 & 1 & 1 & 1 & 1 & 1 & 1 & 1 & 1 & 1 & 1 & 1 & 1 & 1 & 1\end{array}$ $\begin{array}{llllllllllllllllllll}1 & 1 & 1 & 1 & 1 & 1 & 1 & 1 & 1 & 1 & 1 & 1 & 1 & 1 & 1 & 1 & 1 & 1 & 1 & 1\end{array}$ $\begin{array}{llllllllllllll}1 & 1 & 1 & 1 & 1 & 1 & 1 & 1 & 1 & 1 & 1 & 1 & 1 & -1\end{array}$ $\begin{array}{lllllllllllllllllll}-1 & 1 & 1 & 1 & 1 & 1 & 1 & 1 & 1 & 1 & 1 & 1 & 1 & 1 & 1 & 1 & 1 & 1 & 1\end{array}$ $\begin{array}{rrrrrrrrrrrrrr}1 & 1 & 1 & 1 & 1 & 1 & 1 & 1 & 1 & 1 & 1 & 1 & 1 & 1 \\ 1 & 1 & 1 & 1 & 1 & 1 & 1 & 1 & 1 & 1 & 1 & 1 & 1 & -1\end{array}$ $\begin{array}{lllllllllllll}-1 & 1 & 1 & 1 & 1 & 1 & 1 & 1 & 1 & 1 & 1 & 1 & 1\end{array}$ $\begin{array}{rrrrrrrrrrrrrr}1 & 1 & 1 & 1 & 1 & 1 & 1 & 1 & 1 & 1 & 1 & 1 & 1 & 1 \\ 1 & 1 & 1 & 1 & 1 & 1 & 1 & 1 & 1 & 1 & 1 & 1 & 1 & -1\end{array}$ $\begin{array}{rrrrrrrrrrrrrr}1 & 1 & 1 & 1 & 1 & 1 & 1 & 1 & 1 & 1 & 1 & 1 & 1 & -1 \\ -1 & 1 & 1 & 1 & 1 & 1 & 1 & 1 & 1 & 1 & 1 & 1 & 1\end{array}$ $\begin{array}{rrrrrrrrrrrrrr}1 & 1 & 1 & 1 & 1 & 1 & 1 & 1 & 1 & 1 & 1 & 1 & 1 & 1 \\ 1 & 1 & 1 & 1 & 1 & 1 & 1 & 1 & 1 & 1 & 1 & 1 & 1 & -1\end{array}$

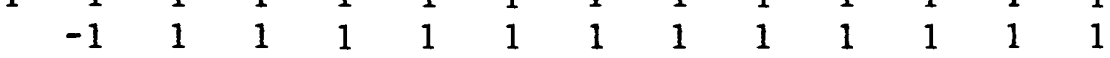
$\begin{array}{rrrrrrrrrrrrrr}1 & 1 & 1 & 1 & 1 & 1 & 1 & 1 & 1 & 1 & 1 & 1 & 1 & 1 \\ 1 & 1 & 1 & 1 & 1 & 1 & 1 & 1 & 1 & 1 & 1 & 1 & 1 & -1\end{array}$ $\begin{array}{rrrrrrrrrrrrrr}1 & 1 & 1 & 1 & 1 & 1 & 1 & 1 & 1 & 1 & 1 & 1 & 1 & -1\end{array}$ $\begin{array}{rrrrrrrrrrrrrr} & -1 & 1 & 1 & 1 & 1 & 1 & 1 & 1 & 1 & 1 & 1 & 1 & 1 \\ 1 & 1 & 1 & 1 & 1 & 1 & 1 & 1 & 1 & 1 & 1 & 1 & 1 & 1\end{array}$ $\begin{array}{rrrrrrrrrrrrrr}1 & 1 & 1 & 1 & 1 & 1 & 1 & 1 & 1 & 1 & 1 & 1 & 1 & -1\end{array}$ $\begin{array}{lllllllllllll}-1 & 1 & 1 & 1 & 1 & 1 & 1 & 1 & 1 & 1 & 1 & 1 & 1\end{array}$ $\begin{array}{rrrrrrrrrrrrrr}1 & 1 & 1 & 1 & 1 & 1 & 1 & 1 & 1 & 1 & 1 & 1 & 1 & 1 \\ 1 & 1 & 1 & 1 & 1 & 1 & 1 & 1 & 1 & 1 & 1 & 1 & 1 & -1\end{array}$ $\begin{array}{rllllllllllllr}1 & 1 & 1 & 1 & 1 & 1 & 1 & 1 & 1 & 1 & 1 & 1 & 1 & -1 \\ & & 1 & 1 & 1 & 1 & 1 & 1 & 1 & 1 & 1 & 1 & 1 & 1\end{array}$ $\begin{array}{rrrrrrrrrrrrrr}1 & 1 & 1 & 1 & 1 & 1 & 1 & 1 & 1 & 1 & 1 & 1 & 1 & 1 \\ 1 & 1 & 1 & 1 & 1 & 1 & 1 & 1 & 1 & 1 & 1 & 1 & 1 & -1\end{array}$

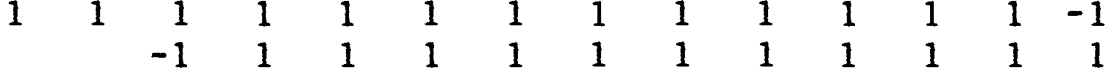
$\begin{array}{rrrrrrrrrrrrrr}1 & 1 & 1 & 1 & 1 & 1 & 1 & 1 & 1 & 1 & 1 & 1 & 1 & 1 \\ 1 & 1 & 1 & 1 & 1 & 1 & 1 & 1 & 1 & 1 & 1 & 1 & 1 & -1\end{array}$ $\begin{array}{rrrrrrrrrrrrrr}1 & 1 & 1 & 1 & 1 & 1 & 1 & 1 & 1 & 1 & 1 & 1 & 1 & -1 \\ & -1 & 1 & 1 & 1 & 1 & 1 & 1 & 1 & 1 & 1 & 1 & 1 & 1\end{array}$ $\begin{array}{rrrrrrrrrrrrrr}1 & 1 & 1 & 1 & 1 & 1 & 1 & 1 & 1 & 1 & 1 & 1 & 1 & 1 \\ 1 & 1 & 1 & 1 & 1 & 1 & 1 & 1 & 1 & 1 & 1 & 1 & 1 & -1\end{array}$ $\begin{array}{rrrrrrrrrrrrrr}1 & 1 & 1 & 1 & 1 & 1 & 1 & 1 & 1 & 1 & 1 & 1 & 1 & -1 \\ & -1 & 1 & 1 & 1 & 1 & 1 & 1 & 1 & 1 & 1 & 1 & 1 & 1\end{array}$ $\begin{array}{llllllllllllllllllll}1 & 1 & 1 & 1 & 1 & 1 & 1 & 1 & 1 & 1 & 1 & 1 & 1 & 1 & 1 & 1 & 1 & 1 & 1 & 1\end{array}$ $\begin{array}{llllllllllllll}1 & 1 & 1 & 1 & 1 & 1 & 1 & 1 & 1 & 1 & 1 & 1 & 1 & -1\end{array}$

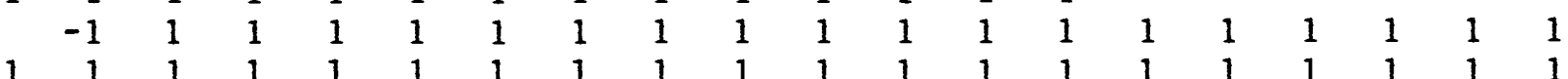
$\begin{array}{rrrrrrrrrrrrrr}1 & 1 & 1 & 1 & 1 & 1 & 1 & 1 & 1 & 1 & 1 & 1 & 1 & 1 \\ 1 & 1 & 1 & 1 & 1 & 1 & 1 & 1 & 1 & 1 & 1 & 1 & 1 & -1\end{array}$ $\begin{array}{llllll}1 & 1 & 1 & 1 & 1 & 1\end{array}$ $\begin{array}{llllll}1 & 1 & 1 & 1 & 1 & 1\end{array}$ $\begin{array}{llllll}1 & 1 & 1 & 1 & 1 & 1\end{array}$ $\begin{array}{lllllllllllll}-1 & 1 & 1 & 1 & 1 & 1 & 1 & 1 & 1 & 1 & 1 & 1 & 1\end{array}$ 
Table 3.--Listing of data for 1971-77--Continued

Card

number
Group III: Array data--Continued

Storage coefficient, dimensionless--Continued
986.

987.

988.

989.

990.

991.

992.

993.

994.

995.

996.

997.

998.

999.

1000 .

1001 .

1002 .

1003.

1004.

1005.

1006.

1007.

1008 .

1009 .

1010 .

1011.

1012.

1013.

1014.

1015.

1016.

1017.

1018 .

1019.

1020 .

1021.

1022.

1023.

1024.

1025.

1026.

1027.

1028 .

1029 .

1030 .

1031. $\begin{array}{rrrrrrrrrrrrrr}1 & 1 & 1 & 1 & 1 & 1 & 1 & 1 & 1 & 1 & 1 & 1 & 1 & 1 \\ 1 & 1 & 1 & 1 & 1 & 1 & 1 & 1 & 1 & 1 & 1 & 1 & 1 & -1\end{array}$

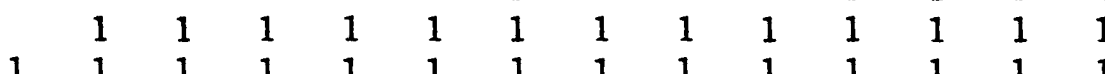

$\begin{array}{rrrrrrrrrrrrrr}1 & 1 & 1 & 1 & 1 & 1 & 1 & 1 & 1 & 1 & 1 & 1 & 1 & 1 \\ 1 & 1 & 1 & 1 & 1 & 1 & 1 & 1 & 1 & 1 & 1 & 1 & 1 & -1\end{array}$

$\begin{array}{llll}1 & 1 & 1\end{array}$

$\begin{array}{llll}1 & 1 & 1 & 1\end{array}$

$\begin{array}{lllll}1 & 1 & 1 & 1 & 1\end{array}$

$\begin{array}{llll}1 & 1 & 1 & 1 \\ 1 & 1 & 1 & 1\end{array}$

$\begin{array}{llll}1 & 1 & 1 & 1 \\ 1 & 1 & 1 & 1\end{array}$

$\begin{array}{lllll}1 & 1 & 1 & 1 & 1\end{array}$

1

1
1

1

1

1

11

11

1

$\begin{array}{rrrrrr}1 & 1 & 1 & 1 & 1 & -1 \\ 1 & 1 & 1 & 1 & 1 & 1\end{array}$

$\begin{array}{rrrrrrrr}1 & 1 & 1 & 1 & 1 & 1 & 1 & 1 \\ 1 & 1 & 1 & 1 & 1 & 1 & 1 & -1\end{array}$

$\begin{array}{llll}1 & 1 & 1 & 1 \\ 1 & 1 & 1 & 1\end{array}$

$\begin{array}{llll}1 & 1 & 1 & 1 \\ 1 & 1 & 1\end{array}$

$\begin{array}{lll}1 & 1 & 1 \\ 1 & 1 & 1\end{array}$

$\begin{array}{llll}1 & 1 & 1\end{array}$ $\begin{array}{cccc}-1 & 1 & 1 & 1\end{array}$ $\begin{array}{lll}1 & 1 & 1\end{array}$ $\begin{array}{rrr}1 & 1 & 1\end{array}$

$\begin{array}{rrrr}1 & -1 & 1 \\ 1 & 1 & 1\end{array}$

$\begin{array}{rrrrrrrrrrrrrr}1 & 1 & 1 & 1 & 1 & 1 & 1 & 1 & 1 & 1 & 1 & 1 & 1 & -1\end{array}$

$\begin{array}{rrrrrrrrrrrrrr} & -1 & 1 & 1 & 1 & 1 & 1 & 1 & 1 & 1 & 1 & 1 & 1 & 1 \\ 1 & 1 & 1 & 1 & 1 & 1 & 1 & 1 & 1 & 1 & 1 & 1 & 1 & 1\end{array}$

$\begin{array}{llllllllllllll}1 & 1 & 1 & 1 & 1 & 1 & 1 & 1 & 1 & 1 & 1 & 1 & 1 & -1\end{array}$

$\begin{array}{rrrrrrrrrrrrrr} & & -1 & 1 & 1 & 1 & 1 & 1 & 1 & 1 & 1 & 1 & 1 & 1 \\ 1 & 1 & 1 & 1 & 1 & 1 & 1 & 1 & 1 & 1 & 1 & 1 & 1 & 1 \\ 1 & 1 & 1 & 1 & 1 & 1 & 1 & 1 & 1 & 1 & 1 & 1 & 1 & -1\end{array}$

$\begin{array}{rrrrrrrrrrrrrr}1 & 1 & 1 & 1 & 1 & 1 & 1 & 1 & 1 & 1 & 1 & 1 & 1 & -1 \\ & & -1 & 1 & 1 & 1 & 1 & 1 & 1 & 1 & 1 & 1 & 1 & 1\end{array}$

$\begin{array}{rrrrrrrrrrrrrr}1 & 1 & 1 & 1 & 1 & 1 & 1 & 1 & 1 & 1 & 1 & 1 & 1 & 1 \\ 1 & 1 & 1 & 1 & 1 & 1 & 1 & 1 & 1 & 1 & 1 & 1 & 1 & -1\end{array}$

$\begin{array}{rrrrrrrrrrrrrr}1 & 1 & 1 & 1 & 1 & 1 & 1 & 1 & 1 & 1 & 1 & 1 & 1 & -1 \\ & & & -1 & 1 & 1 & 1 & 1 & 1 & 1 & 1 & 1 & 1 & 1\end{array}$

$\begin{array}{rrrrrrrrrrrrrr}1 & 1 & 1 & 1 & 1 & 1 & 1 & 1 & 1 & 1 & 1 & 1 & 1 & 1 \\ 1 & 1 & 1 & 1 & 1 & 1 & 1 & 1 & 1 & 1 & 1 & 1 & 1 & -1\end{array}$

$\begin{array}{rrrrrrrrrrrrrr}1 & 1 & 1 & 1 & 1 & 1 & 1 & 1 & 1 & 1 & 1 & 1 & 1 & -1 \\ & & & -1 & 1 & 1 & 1 & 1 & 1 & 1 & 1 & 1 & 1 & 1\end{array}$

$\begin{array}{rrrrrrrrrrrrrr}1 & 1 & 1 & 1 & 1 & 1 & 1 & 1 & 1 & 1 & 1 & 1 & 1 & 1 \\ 1 & 1 & 1 & 1 & 1 & 1 & 1 & 1 & 1 & 1 & 1 & 1 & 1 & -1\end{array}$

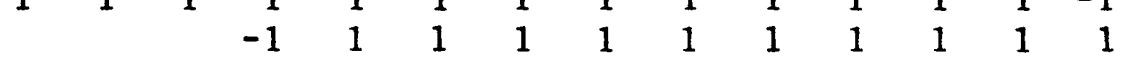

$\begin{array}{rrrrrrrrrrrrrr}1 & 1 & 1 & 1 & 1 & 1 & 1 & 1 & 1 & 1 & 1 & 1 & 1 & 1 \\ 1 & 1 & 1 & 1 & 1 & 1 & 1 & 1 & 1 & 1 & 1 & 1 & 1 & -1\end{array}$

$\begin{array}{rrrrrrrrrrrrrr}1 & 1 & 1 & 1 & 1 & 1 & 1 & 1 & 1 & 1 & 1 & 1 & 1 & -1 \\ & & -1 & 1 & 1 & 1 & 1 & 1 & 1 & 1 & 1 & 1 & 1\end{array}$

$\begin{array}{rrrrrrrrrrrrrr}1 & 1 & 1 & 1 & 1 & 1 & 1 & 1 & 1 & 1 & 1 & 1 & 1 & 1 \\ 1 & 1 & 1 & 1 & 1 & 1 & 1 & 1 & 1 & 1 & 1 & 1 & 1 & -1\end{array}$

$\begin{array}{rrrrrrrrrrrrrrr}1 & 1 & 1 & 1 & 1 & 1 & 1 & 1 & 1 & 1 & 1 & 1 & 1 & -1\end{array}$

$\begin{array}{llllllllllllll}1 & 1 & 1 & 1 & 1 & 1 & 1 & 1 & 1 & 1 & 1 & 1 & 1 & 1\end{array}$

$\begin{array}{llllll}1 & 1 & 1 & 1 & 1 & 1\end{array}$

$\begin{array}{llllll}1 & 1 & 1 & 1 & 1 & 1\end{array}$

$\begin{array}{llllll}1 & 1 & 1 & 1 & 1 & 1\end{array}$

$\begin{array}{llllll}1 & 1 & 1 & 1 & 1 & 1\end{array}$

$\begin{array}{llllll}1 & 1 & 1 & 1 & 1 & 1\end{array}$

$\begin{array}{llllll}1 & 1 & 1 & 1 & 1 & 1\end{array}$

$\begin{array}{llllll}1 & 1 & 1 & 1 & 1 & 1\end{array}$ (1)

$\begin{array}{llllll}1 & 1 & 1 & 1 & 1 & 1\end{array}$ $\begin{array}{llllll}1 & 1 & 1 & 1 & 1 & 1\end{array}$ $\begin{array}{llllll}1 & 1 & 1 & 1 & 1 & 1 \\ 1 & 1 & 1 & 1 & 1 & 1\end{array}$ $\begin{array}{llllll}1 & 1 & 1 & 1 & 1 & 1\end{array}$ $\begin{array}{llllll}1 & 1 & 1 & 1 & 1 & 1\end{array}$ $\begin{array}{llllll}1 & 1 & 1 & 1 & 1 & 1\end{array}$ $\begin{array}{llllll}1 & 1 & 1 & 1 & 1 & 1\end{array}$ $\begin{array}{llllll}1 & 1 & 1 & 1 & 1 & 1 \\ 1 & 1 & 1 & 1 & 1 & 1\end{array}$ $\begin{array}{llllll}1 & 1 & 1 & 1 & 1 & 1\end{array}$ $\begin{array}{llllll}1 & 1 & 1 & 1 & 1 & 1\end{array}$ $\begin{array}{llllll}1 & 1 & 1 & 1 & 1 & 1\end{array}$ $\begin{array}{llllll}1 & 1 & 1 & 1 & 1 & 1\end{array}$ $\begin{array}{llllll}1 & 1 & 1 & 1 & 1 & 1\end{array}$ $\begin{array}{llllll}1 & 1 & 1 & 1 & 1 & 1\end{array}$ $\begin{array}{llllll}1 & 1 & 1 & 1 & 1 & 1 \\ 1 & 1 & 1 & 1 & 1 & 1\end{array}$ $\begin{array}{llllll}1 & 1 & 1 & 1 & 1 & 1\end{array}$ $\begin{array}{llllll}1 & 1 & 1 & 1 & 1 & 1\end{array}$ $\begin{array}{llllll}1 & 1 & 1 & 1 & 1 & 1 \\ 1 & 1 & 1 & 1 & 1 & 1\end{array}$ 
Table 3.--Listing of data for 1971-77--Continued

Card number

1032 1033. 1034 . 1035 . 1036. 1037. 1038 1039. 1040. 1041 1042. 1043. 1044 . 1045 . 1046. 1047. 1048. 1049. 1050. 1051. 1052. 1053. 1054. 1055. 1056. 1057. 1058. 1059. 1060. 1061. 1062. 1063. 1064. 1065. 1066. 1067. 1068. 1069. 1070. 1071. 1072. 1073. 1074. 1075. 1076. 1077. 1078. 1079. 1080.
Group III: Array data--Continued Storage coefficient, dimensionless--Continued $\begin{array}{rrrrrrrrrrrrrr}1 & 1 & 1 & 1 & 1 & 1 & 1 & 1 & 1 & 1 & 1 & 1 & 1 & -1 \\ & & -1 & 1 & 1 & 1 & 1 & 1 & 1 & 1 & 1 & 1 & 1 & 1\end{array}$

$\begin{array}{rllllllllllllr}1 & 1 & 1 & 1 & 1 & 1 & 1 & 1 & 1 & 1 & 1 & 1 & 1 & 1 \\ 1 & 1 & 1 & 1 & 1 & 1 & 1 & 1 & 1 & 1 & 1 & 1 & 1 & -1\end{array}$

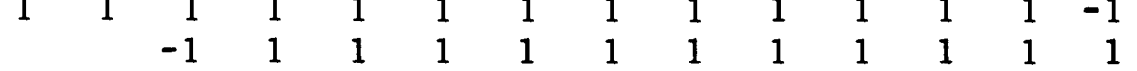

$\begin{array}{rllllllllllllr}1 & 1 & 1 & 1 & 1 & 1 & 1 & 1 & 1 & 1 & 1 & 1 & 1 & 1 \\ 1 & 1 & 1 & 1 & 1 & 1 & 1 & 1 & 1 & 1 & 1 & 1 & 1 & -1\end{array}$

$\begin{array}{lllllllllllllllllll}1 & -1 & 1 & 1 & 1 & 1 & 1 & 1 & 1 & 1 & 1 & 1 & 1 & 1 & 1 & 1 & 1 & 1 & 1\end{array}$

$\begin{array}{llllllllllllllllllll}1 & 1 & 1 & 1 & 1 & 1 & 1 & 1 & 1 & 1 & 1 & 1 & 1 & 1 & 1 & 1 & 1 & 1 & 1 & 1\end{array}$

$\begin{array}{llllllllllllll}1 & 1 & 1 & 1 & 1 & 1 & 1 & 1 & 1 & 1 & 1 & 1 & 1 & -1\end{array}$

$\begin{array}{lllllllllllllllllll}-1 & 1 & 1 & 1 & 1 & 1 & 1 & 1 & 1 & 1 & 1 & 1 & 1 & 1 & 1 & 1 & 1 & 1 & 1\end{array}$

$\begin{array}{llllllllllllllllllll}1 & 1 & 1 & 1 & 1 & 1 & 1 & 1 & 1 & 1 & 1 & 1 & 1 & 1 & 1 & 1 & 1 & 1 & 1 & 1\end{array}$

$\begin{array}{rrrrrrrrrrrrrr}1 & 1 & 1 & 1 & 1 & 1 & 1 & 1 & 1 & 1 & 1 & 1 & 1 & -1\end{array}$

$\begin{array}{lllllllllllll}-1 & 1 & 1 & 1 & 1 & 1 & 1 & 1 & 1 & 1 & 1 & 1 & 1\end{array}$

$\begin{array}{llllllllllllll}1 & 1 & 1 & 1 & 1 & 1 & 1 & 1 & 1 & 1 & 1 & 1 & 1 & 1\end{array}$

$\begin{array}{llllllllllllll}1 & 1 & 1 & 1 & 1 & 1 & 1 & 1 & 1 & 1 & 1 & 1 & 1 & -1\end{array}$

$\begin{array}{lllllllllllllllllll}-1 & 1 & 1 & 1 & 1 & 1 & 1 & 1 & 1 & 1 & 1 & 1 & 1 & 1 & 1 & 1 & 1 & 1 & 1\end{array}$

$\begin{array}{llllllllllllllllllll}1 & 1 & 1 & 1 & 1 & 1 & 1 & 1 & 1 & 1 & 1 & 1 & 1 & 1 & 1 & 1 & 1 & 1 & 1 & 1\end{array}$

$\begin{array}{llllllllllllll}1 & 1 & 1 & 1 & 1 & 1 & 1 & 1 & 1 & 1 & 1 & 1 & 1 & -1\end{array}$

$\begin{array}{lllllllllllllllllll}-1 & 1 & 1 & 1 & 1 & 1 & 1 & 1 & 1 & 1 & 1 & 1 & 1 & 1 & 1 & 1 & 1 & 1 & 1\end{array}$

$\begin{array}{llllllllllllllllllll}1 & 1 & 1 & 1 & 1 & 1 & 1 & 1 & 1 & 1 & 1 & 1 & 1 & 1 & 1 & 1 & 1 & 1 & 1 & 1\end{array}$

$\begin{array}{llllllllllllll}1 & 1 & 1 & 1 & 1 & 1 & 1 & 1 & 1 & 1 & 1 & 1 & 1 & -1\end{array}$

$\begin{array}{lllllllllllllllllll}-1 & 1 & 1 & 1 & 1 & 1 & 1 & 1 & 1 & 1 & 1 & 1 & 1 & 1 & 1 & 1 & 1 & 1 & 1\end{array}$

$\begin{array}{llllllllllllllllllll}1 & 1 & 1 & 1 & 1 & 1 & 1 & 1 & 1 & 1 & 1 & 1 & 1 & 1 & 1 & 1 & 1 & 1 & 1 & 1\end{array}$

$\begin{array}{llllllllllllll}1 & 1 & 1 & 1 & 1 & 1 & 1 & 1 & 1 & 1 & 1 & 1 & 1 & -1\end{array}$

$\begin{array}{lllllllllllllllllll}-1 & 1 & 1 & 1 & 1 & 1 & 1 & 1 & 1 & 1 & 1 & 1 & 1 & 1 & 1 & 1 & 1 & 1 & 1\end{array}$

$\begin{array}{llllllllllllllllllll}1 & 1 & 1 & 1 & 1 & 1 & 1 & 1 & 1 & 1 & 1 & 1 & 1 & 1 & 1 & 1 & 1 & 1 & 1 & 1\end{array}$

$\begin{array}{llllllllllllll}1 & 1 & 1 & 1 & 1 & 1 & 1 & 1 & 1 & 1 & 1 & 1 & 1 & -1\end{array}$

$$
\begin{array}{lllllllllllllllllll}
-1 & 1 & 1 & 1 & 1 & 1 & 1 & 1 & 1 & 1 & 1 & 1 & 1 & 1 & 1 & 1 & 1 & 1 & 1
\end{array}
$$

$\begin{array}{llllllllllllllllllll}1 & 1 & 1 & 1 & 1 & 1 & 1 & 1 & 1 & 1 & 1 & 1 & 1 & 1 & 1 & 1 & 1 & 1 & 1 & 1\end{array}$

$\begin{array}{llllllllllllll}1 & 1 & 1 & 1 & 1 & 1 & 1 & 1 & 1 & 1 & 1 & 1 & 1 & -1\end{array}$

$\begin{array}{lllllllllllllllllll}-1 & 1 & 1 & 1 & 1 & 1 & 1 & 1 & 1 & 1 & 1 & 1 & 1 & 1 & 1 & 1 & 1 & 1 & 1\end{array}$

$\begin{array}{llllllllllllllllllll}1 & 1 & 1 & 1 & 1 & 1 & 1 & 1 & 1 & 1 & 1 & 1 & 1 & 1 & 1 & 1 & 1 & 1 & 1 & 1\end{array}$

$\begin{array}{llllllllllllll}1 & 1 & 1 & 1 & 1 & 1 & 1 & 1 & 1 & 1 & 1 & 1 & 1 & -1\end{array}$

$\begin{array}{lllllllllllllllllll}-1 & 1 & 1 & 1 & 1 & 1 & 1 & 1 & 1 & 1 & 1 & 1 & 1 & 1 & 1 & 1 & 1 & 1 & 1\end{array}$

$\begin{array}{llllllllllllllllllll}1 & 1 & 1 & 1 & 1 & 1 & 1 & 1 & 1 & 1 & 1 & 1 & 1 & 1 & 1 & 1 & 1 & 1 & 1 & 1\end{array}$

$\begin{array}{llllllllllllll}1 & 1 & 1 & 1 & 1 & 1 & 1 & 1 & 1 & 1 & 1 & 1 & 1 & -1\end{array}$

$\begin{array}{lllllllllllllllllll}-1 & 1 & 1 & 1 & 1 & 1 & 1 & 1 & 1 & 1 & 1 & 1 & 1 & 1 & 1 & 1 & 1 & 1 & 1\end{array}$

\begin{tabular}{lllllllllll|lllllllll}
1 & 1 & 1 & 1 & 1 & 1 & 1 & 1 & 1 & 1 & 1 & 1 & 1 & 1 & 1 & 1 & 1 & 1 & 1 & 1
\end{tabular}

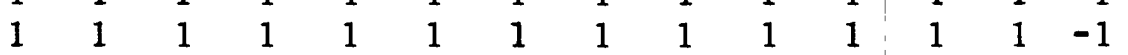

\begin{tabular}{llllllllll|llllllllll}
-1 & 1 & 1 & 1 & 1 & 1 & 1 & 1 & 1 & 1 & 1 & 1 & 1 & 1 & 1 & 1 & 1 & 1 & 1
\end{tabular}

$\begin{array}{llllllllllllllllllll}1 & 1 & 1 & 1 & 1 & 1 & 1 & 1 & 1 & 1 & 1 & 1 & 1 & 1 & 1 & 1 & 1 & 1 & 1 & 1\end{array}$

$\begin{array}{llllllllllllll}1 & 1 & 1 & 1 & 1 & 1 & 1 & 1 & 1 & 1 & 1 & 1 & 1 & -1\end{array}$

$\begin{array}{lllllllllllllllllll}-1 & 1 & 1 & 1 & 1 & 1 & 1 & 1 & 1 & 1 & 1 & 1 & 1 & 1 & 1 & 1 & 1 & 1 & 1\end{array}$

$\begin{array}{llllllllllllllllllll}1 & 1 & 1 & 1 & 1 & 1 & 1 & 1 & 1 & 1 & 1 & 1 & 1 & 1 & 1 & 1 & 1 & 1 & 1 & 1\end{array}$

$\begin{array}{llllllllllllll}1 & 1 & 1 & 1 & 1 & 1 & 1 & 1 & 1 & 1 & 1 & 1 & 1 & -1\end{array}$

$\begin{array}{lllllllllllllllllll}-1 & 1 & 1 & 1 & 1 & 1 & 1 & 1 & 1 & 1 & 1 & 1 & 1 & 1 & 1 & 1 & 1 & 1 & 1\end{array}$

$\begin{array}{llllllllllllllllllll}1 & 1 & 1 & 1 & 1 & 1 & 1 & 1 & 1 & 1 & 1 & 1 & 1 & 1 & 1 & 1 & 1 & 1 & 1 & 1\end{array}$ 
Table 3.--Listing of data for 1971-77--Continued

Card number

1081 . 1082 . 1083 . 1084 . 1085 . 1086.
Group III: Array data--Continued

Storage coefficient, dimensionless--Continued

$$
\begin{array}{lllllllllllllllllllll} 
& -1 & -1 & -1 & -1 & -1 & -1 & -1 & -1 & -1 & -1 & -1 & -1 & -1 & -1 & -1 & -1 & -1 & -1 & -1 \\
-1 & -1 & -1 & -1 & -1 & -1 & -1 & -1 & -1 & -1 & -1 & -1 & -1 & -1 & -1 & -1 & -1 & -1 & -1 & -1 \\
-1 & -1 & -1 & -1 & -1 & -1 & -1 & -1 & -1 & -1 & -1 & -1 & -1 & -1 & & & & & &
\end{array}
$$

Group III: Array data--Continued

Hydraulic conductivity, in feet per second

(First card is parameter card)

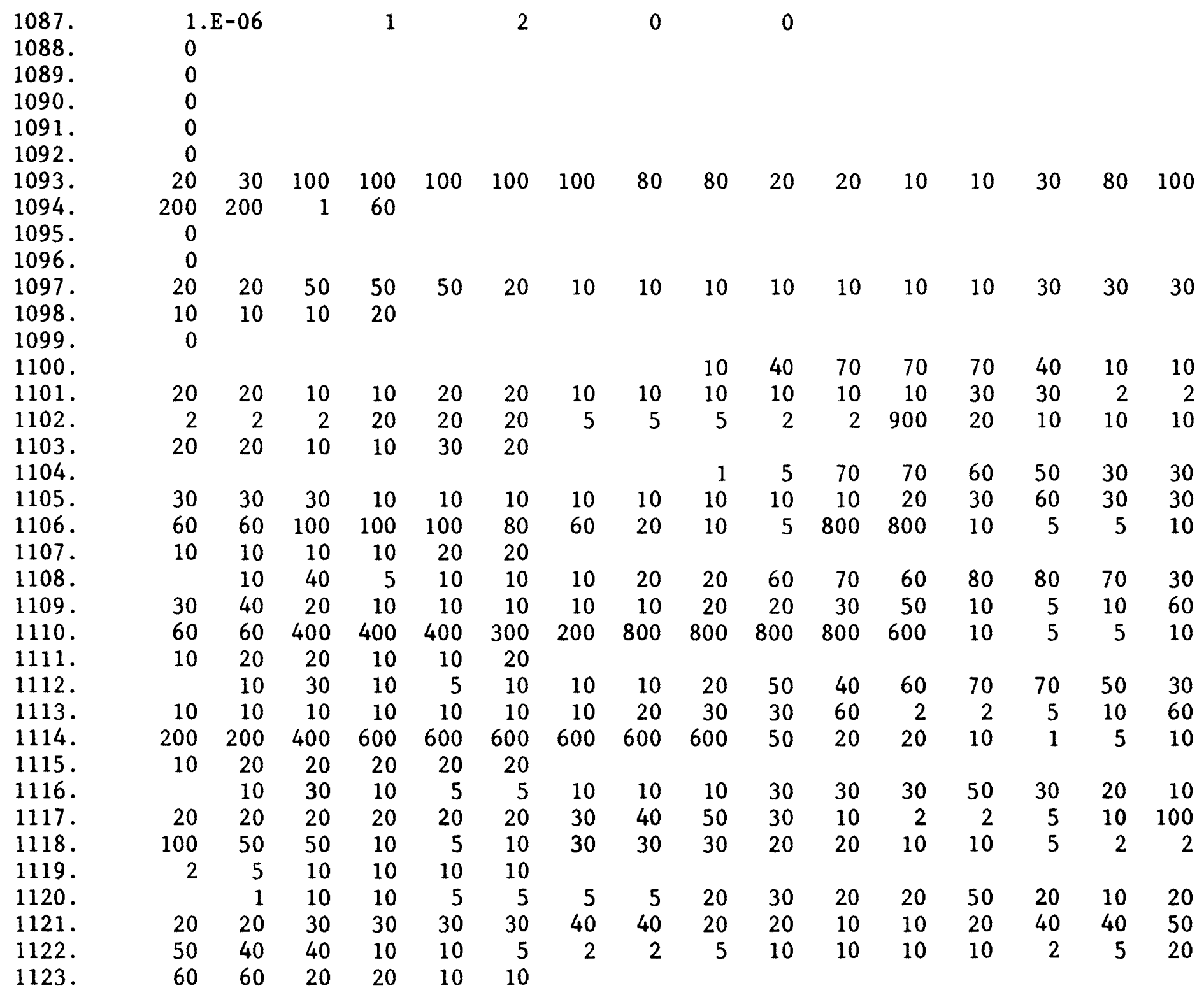


Table 3.--Listing of data for 1971-77--Continued

Card

aumber

1124.

1125 .

1126.

1127.

1128 .

1129

1130 .

1131 .

1132 .

1133.

1134.

1135 .

1136.

1137 .

1138.

1139.

1140 .

1141.

1142.

1143.

1144.

1145.

1146.

1147.

1148.

1149.

1150.

1151.

1152.

1153.

1154.

1155.

1156.

1157.

1158.

1159.

1160.

1161.

1162.

1163.

1164.

1165.

1166.

1167.

1168.

1169.

1170.

1171.

1172.
Group III: Array data--Continued

Hydraulic conductivity, in feet per second--Continued

\begin{tabular}{|c|c|c|c|c|c|c|c|c|c|c|c|c|c|c|c|}
\hline & 1 & 5 & 5 & 5 & 5 & 10 & 10 & 30 & 20 & 10 & 10 & 10 & 10 & 10 & 20 \\
\hline 20 & 20 & 10 & 10 & 20 & 20 & 20 & 20 & 20 & 20 & 20 & 20 & 20 & 20 & 20 & 40 \\
\hline 40 & 20 & 20 & 10 & 10 & 1 & 1 & 1 & 5 & 10 & 10 & 10 & 10 & 10 & 20 & 50 \\
\hline 80 & 80 & 40 & 40 & 20 & 20 & & & & & & & & & & \\
\hline & 1 & 5 & 20 & 5 & 5 & 10 & 10 & 40 & 20 & 10 & 20 & 20 & 20 & 20 & 20 \\
\hline 20 & 20 & 10 & 10 & 20 & 20 & 20 & 20 & 20 & 20 & 10 & 20 & 30 & 40 & 30 & 0 \\
\hline 10 & 10 & 5 & 5 & 1 & 1 & 1 & 2 & 5 & 5 & 5 & 5 & 10 & 10 & 20 & 0 \\
\hline 70 & 50 & 50 & 60 & 30 & 30 & & & & & & & & & & \\
\hline & 1 & 5 & 20 & 20 & 10 & 30 & 10 & 10 & 5 & 5 & 10 & 10 & 10 & 10 & \\
\hline 20 & 20 & 10 & 10 & 20 & 20 & 20 & 20 & 40 & 40 & 40 & 40 & 40 & 40 & 30 & \\
\hline 40 & 40 & 40 & 40 & 30 & 30 & 30 & 30 & 50 & 50 & 50 & 70 & 70 & 80 & 80 & \\
\hline
\end{tabular}

$\begin{array}{llllll}60 & 50 & 10 & 60 & 80 & 80\end{array}$

$1 \quad 10 \quad 20 \quad 30 \quad 30$

$\begin{array}{llllllllll}30 & 5 & 5 & 5 & 10 & 10 & 10 & 10 & 10 & 10\end{array}$

$\begin{array}{llllll}10 & 10 & 10 & 10 & 20 & 20\end{array}$

$\begin{array}{llllll}50 & 50 & 50 & 40 & 40 & 40 \\ 10 & 10 & 10 & 50 & 90 & 90\end{array}$

$\begin{array}{llll}20 & 20 & 10 & 5\end{array}$

$\begin{array}{llllllllll}30 & 40 & 60 & 60 & 60 & 60 & 60 & 60 & 60 & 50\end{array}$

$\begin{array}{llllllllll}40 & 40 & 50 & 50 & 60 & 70 & 80 & 80 & 80 & 60\end{array}$

$\begin{array}{llllll}10 & 10 & 10 & 20 & 20 & 30\end{array}$

$\begin{array}{llllll}60 & 50 & 50 & 50 & 50 & 50\end{array}$

$\begin{array}{llllll}20 & 10 & 10 & 40 & 100 & 500\end{array}$

$\begin{array}{llll}20 & 5 & 10 & 10\end{array}$

$\begin{array}{llllll}10 & 10 & 20 & 30 & 30 & 40\end{array}$

4060

$\begin{array}{llllllllll}10 & 5 & 5 & 5 & 2 & 5 & 5 & 5 & 5 & 5\end{array}$

$\begin{array}{llllllllll}40 & 60 & 60 & 60 & 60 & 60 & 60 & 60 & 60 & 60\end{array}$

$\begin{array}{llllllllll}50 & 50 & 50 & 50 & 80 & 100 & 200 & 400 & 60 & 60\end{array}$

$\begin{array}{llllll}60 & 100 & 100 & 100 & 100 & 100\end{array}$

$\begin{array}{llllll}20 & 20 & 20 & 100 & 500 & 500\end{array}$

$$
\begin{array}{lrrr}
20 & 5 & 5 & 5
\end{array}
$$

$\begin{array}{llllll}10 & 20 & 30 & 30 & 40 & 50\end{array}$

$\begin{array}{rrrrrr}100 & 100 & 100 & 200 & 200 & 200\end{array}$

$\begin{array}{llllllllll}10 & 5 & 5 & 2 & 2 & 2 & 10 & 10 & 10 & 10\end{array}$ $\begin{array}{rrrrrrrrrr}60 & 60 & 60 & 60 & 60 & 60 & 60 & 60 & 60 & 60\end{array}$ $\begin{array}{llllll}500 & 500 & 500 & 500 & 500 & 500\end{array}$

$$
\begin{array}{rrrr}
40 & 10 & 5 & 5
\end{array}
$$

$\begin{array}{llllll}30 & 30 & 30 & 30 & 40 & 50\end{array}$

$\begin{array}{cccccc}100 & 200 & 200 & 200 & 200 & 200\end{array}$

$\begin{array}{llllll}500 & 500 & 500 & 500 & 500 & 500\end{array}$

$$
\begin{array}{lllll}
40 & 2 & 10 & 10
\end{array}
$$

$\begin{array}{rrrrrr}30 & 30 & 30 & 30 & 40 & 40 \\ 200 & 200 & 200 & 200 & 200 & 200\end{array}$

$\begin{array}{rrrrrrrrrrr}5 & 2 & 10 & 5 & 2 & 2 & 10 & 10 & 20 & 30 & 30\end{array}$

$\begin{array}{llllll}200 & 200 & 200 & 200 & 200 & 200 \\ 500 & 500 & 500 & 500 & 500 & 500\end{array}$

500

$\begin{array}{rrrrr}40 & 10 & 10 & 5 & 5 \\ 20 & 20 & 20 & 20 & 30\end{array}$

$\begin{array}{rrrrrr}30 & 20 & 20 & 20 & 20 & 30 \\ 200 & 200 & 200 & 200 & 200 & 200\end{array}$

$\begin{array}{llllll}400 & 400 & 400 & 300 & 400 & 400\end{array}$

$\begin{array}{lllllllllllllll}20 & 10 & 20 & 10 & 10 & 10 & 20 & 20 & 20 & 20 & 10 & 20 & 30 & 30 & 30\end{array}$

$\begin{array}{llllllllllllllll}30 & 30 & 50 & 50 & 50 & 50 & 100 & 100 & 100 & 100 & 100 & 200 & 200 & 200 & 200 & 100\end{array}$

$\begin{array}{llllllllllllllll}100 & 100 & 100 & 100 & 80 & 80 & 100 & 100 & 100 & 100 & 100 & 100 & 100 & 100 & 200 & 400\end{array}$

$400 \quad 400 \quad 300 \quad 200 \quad 200 \quad 400$

$\begin{array}{lllllllllllllll}30 & 30 & 10 & 10 & 10 & 10 & 10 & 20 & 20 & 20 & 10 & 20 & 30 & 50 & 50\end{array}$

$\begin{array}{rrrrrrrrrrrrrrrr}50 & 90 & 90 & 90 & 100 & 100 & 100 & 100 & 200 & 200 & 200 & 200 & 200 & 200 & 200 & 100\end{array}$

$\begin{array}{rrrrrrrrrrrrrrrr}100 & 100 & 100 & 80 & 80 & 80 & 80 & 100 & 100 & 100 & 100 & 100 & 100 & 100 & 100 & 100\end{array}$

$\begin{array}{llllll}100 & 90 & 100 & 100 & 100 & 100\end{array}$

$\begin{array}{lllllllllllllll}40 & 20 & 10 & 10 & 10 & 10 & 20 & 20 & 20 & 20 & 80 & 80 & 50 & 50 & 50\end{array}$ 
Table 3.--Listing of data for 1971-77--Continued

Card number

1173.

1174.

1175 .

1176.

1177.

1178 .

1179 .

1180 .

1181 .

1182 .

1183.

1184 .

1185 .

1186.

1187 .

1188 .

1189 .

1190 .

1191.

1192.

1193.

1194.

1195.

1196.

1197.

1198 .

1199 .

1200 .

1201.

1202.

1203.

1204.

1205 .

1206 .

1207.

1208 .

1209 .

1210 .

1211.

1212.

1213.

1214 .

1215 .

1216.

1217.

1218 .

1219 .

1220 .

1221 .

Group III: Array data--Continued

Hydraulic conductivity, in feet per second--Continued

100

$50 \quad 90$

$\begin{array}{rrrr}100 & 100 & 100 & 90\end{array}$

$\begin{array}{llll}20 & 20 & 20 & 20\end{array}$

$30 \quad 10 \quad 10$

$\begin{array}{rrrr}70 & 80 & 100 & 100\end{array}$

$\begin{array}{rrrr}100 & 100 & 90 & 80\end{array}$

$\begin{array}{llll}60 & 30 & 20 & 20\end{array}$

$\begin{array}{rrr}10 & 20 & 30 \\ 100 & 100 & 100\end{array}$

$\begin{array}{rrrr}80 & 100 & 100 & 100 \\ 90 & 90 & 90 & \end{array}$

$50 \quad 30 \quad 20 \quad 20$

$10 \quad 20 \quad 30$

$\begin{array}{lrrrr}100 & 100 & 100 & 100 & 100\end{array}$

$\begin{array}{lllll}90 & 90 & 90 & 70 & 70\end{array}$

$\begin{array}{lllll}20 & 20 & 20 & 20 & 50\end{array}$

$\begin{array}{llll}20 & 10 & 10 & 10\end{array}$

$\begin{array}{rrrrrr}100 & 100 & 100 & 100 & 100 & 10\end{array}$

$\begin{array}{llllll}40 & 30 & 20 & 20 & 20 & 20\end{array}$

$\begin{array}{llllll}20 & 20 & 20 & 20 & 50 & 50\end{array}$

$\begin{array}{rrrrr}400 & 50 & 30 & 5 & 2\end{array}$

$\begin{array}{rrrrrr}70 & 100 & 100 & 100 & 100 & 10\end{array}$

$\begin{array}{llllll}40 & 40 & 20 & 20 & 20 & 20\end{array}$

$\begin{array}{llllll}20 & 20 & 20 & 20 & 80 & 80\end{array}$

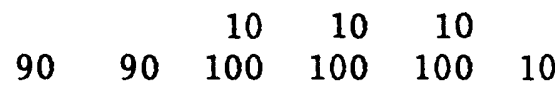

$\begin{array}{llllll}90 & 90 & 90 & 70 & 60 & 60\end{array}$

$\begin{array}{llllll}20 & 20 & 40 & 90 & 2500 & 3500\end{array}$

$\begin{array}{rrrrrr}90 & 100 & 100 & 200 & 200 & 100\end{array}$

$\begin{array}{llrrrr}90 & 90 & 90 & 70 & 60 & 60\end{array}$

$400040003800 \quad 40004000 \quad 20$

$\begin{array}{lll}10 & 10 & 20\end{array}$

$\begin{array}{llllll}90 & 100 & 100 & 200 & 200 & 200\end{array}$

$\begin{array}{llllll}200 & 200 & 200 & 200 & 100 & 100\end{array}$

5000400030003000

10

$\begin{array}{llll}100 & 100 & 200 & 200\end{array}$

$\begin{array}{llll}200 & 300 & 300 & 200\end{array}$

$\begin{array}{llll}2300 & 20 & 10 & 10\end{array}$

$\begin{array}{rrrrr}100 & 200 & 200 & 5 & \end{array}$

$200 \quad 300 \quad 300 \quad 200 \quad 200$

$\begin{array}{lrrrr}2 & 5 & 10 & 10 & 100\end{array}$

5

200

200

50

55

$200 \quad 200$

100

400

$\begin{array}{rr}5 & 2 \\ 100 & 100\end{array}$

$\begin{array}{llllll}100 & 100 & 100 & 100 & 100 & 100\end{array}$

$\begin{array}{llllll}200 & 300 & 300 & 200 & 300 & 400\end{array}$

$\begin{array}{lllrrr}1 & 10 & 20 & 20 & 80 & 80\end{array}$

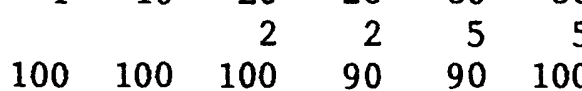

$\begin{array}{llllllllll}100 & 200 & 200 & 100 & 100 & 100 & 100 & 100 & 100 & 100\end{array}$

$\begin{array}{llllllllll}50 & 80 & 90 & 90 & 90 & 200 & 100 & 100 & 50 & 40\end{array}$

$\begin{array}{rrrrrrrrrr}5 & 10 & 20 & 20 & 20 & 20 & 30 & 60 & 60 & 60\end{array}$ $\begin{array}{llllllllll}100 & 100 & 100 & 100 & 100 & 100 & 100 & 100 & 100 & 100\end{array}$ $\begin{array}{llllllllll}50 & 50 & 70 & 90 & 90 & 100 & 100 & 100 & 100 & 80\end{array}$

$\begin{array}{llllllllll}10 & 10 & 20 & 20 & 10 & 10 & 20 & 30 & 80 & 70\end{array}$ $\begin{array}{rrrrrrrrrr}100 & 100 & 100 & 100 & 100 & 100 & 100 & 100 & 100 & 100\end{array}$ $\begin{array}{llllllllll}50 & 50 & 70 & 80 & 80 & 80 & 100 & 100 & 60 & 60\end{array}$

$\begin{array}{llllllllll}5 & 20 & 20 & 20 & 20 & 10 & 20 & 20 & 20 & 20\end{array}$

$\begin{array}{rrrrrrrrrr}200 & 200 & 200 & 200 & 200 & 200 & 200 & 200 & 100 & 90\end{array}$

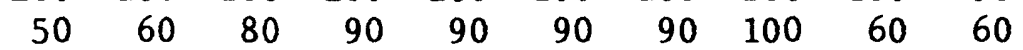

$\begin{array}{llllllllll}5 & 5 & 10 & 10 & 10 & 10 & 20 & 30 & 50 & 60\end{array}$ $\begin{array}{llllllllll}100 & 100 & 100 & 60 & 60 & 60 & 60 & 60 & 40 & 40\end{array}$ $\begin{array}{llllllllll}20 & 30 & 80 & 80 & 90 & 90 & 90 & 90 & 40 & 20\end{array}$

$\begin{array}{llllllllll}1 & 10 & 10 & 10 & 5 & 10 & 20 & 40 & 60 & 60\end{array}$ $\begin{array}{llllllllll}100 & 100 & 100 & 60 & 60 & 60 & 60 & 60 & 40 & 40\end{array}$ $\begin{array}{llllllllll}20 & 30 & 200 & 200 & 400 & 800 & 600 & 200 & 90 & 20\end{array}$

$\begin{array}{llllllllll}1 & 2 & 2 & 5 & 5 & 10 & 20 & 50 & 60 & 60\end{array}$ $\begin{array}{rrrrrrrrrr}100 & 100 & 100 & 60 & 60 & 80 & 80 & 80 & 80 & 90\end{array}$ $\begin{array}{llllllllll}80 & 100 & 200 & 200 & 600 & 800 & 800 & 800 & 300 & 50\end{array}$

$\begin{array}{llllllllll}1 & 2 & 5 & 2 & 10 & 10 & 20 & 30 & 40 & 60\end{array}$ $\begin{array}{llllllllll}90 & 90 & 80 & 80 & 80 & 80 & 90 & 90 & 90 & 90\end{array}$ $\begin{array}{llllllllll}60 & 90 & 200 & 200 & 200 & 200 & 800 & 800 & 4000 & 4000\end{array}$

$\begin{array}{llllllllll}20 & 5 & 5 & 5 & 5 & 10 & 20 & 30 & 30 & 60\end{array}$ $\begin{array}{llllllllll}200 & 100 & 200 & 200 & 200 & 200 & 200 & 200 & 200 & 200\end{array}$ $\begin{array}{llllllllll}100 & 200 & 300 & 300 & 200 & 200 & 300 & 300 & 4000 & 4000\end{array}$

$\begin{array}{lllllllllll}5 & 30 & 30 & 10 & 10 & 5 & 10 & 20 & 30 & 40 & 90\end{array}$ $\begin{array}{rrrrrrrrrr}200 & 200 & 200 & 200 & 200 & 200 & 100 & 200 & 200 & 200\end{array}$ $\begin{array}{lllllllllll}400 & 300 & 300 & 300 & 300 & 300 & 300 & 700 & 5000 & 5000\end{array}$

$\begin{array}{rrrrrrrrrr}5 & 10 & 10 & 30 & 10 & 10 & 30 & 40 & 80 & 100\end{array}$ $\begin{array}{llllllllll}200 & 200 & 200 & 100 & 100 & 100 & 200 & 200 & 200 & 200\end{array}$ $\begin{array}{llllllllll}400 & 400 & 400 & 400 & 400 & 400 & 300 & 5000 & 5000 & 2\end{array}$

$\begin{array}{lllllllllll}5 & 5 & 5 & 10 & 30 & 10 & 10 & 50 & 70 & 90 & 100\end{array}$ $\begin{array}{llllllllll}200 & 200 & 200 & 100 & 100 & 100 & 200 & 200 & 200 & 200\end{array}$ $\begin{array}{llllllllll}300 & 300 & 400 & 400 & 400 & 400 & 5000 & 5000 & 30 & 2\end{array}$

$\begin{array}{llllllllll}5 & 5 & 5 & 30 & 30 & 40 & 50 & 70 & 80 & 100\end{array}$ $\begin{array}{rrrrrrrrrr}90 & 100 & 100 & 100 & 100 & 100 & 200 & 200 & 200 & 200\end{array}$ 
Table 3.--Listing of data for 1971-77--Continued

Card

umber

1222.

1223.

1224.

1225.

1226.

1227.

1228.

1229.

1230.

1231.

1232.

1233.

1234.

1235.

1236.

1237.

1238.

1239.

1240 .

1241.

1242.

1243.

1244.

1245 .

1246.

1247.

1248.

1249.

1250.

1251.

1252.

1253.

1254.

1255.

1256.

1257.

1258.

1259.

1260.

1261.

1262.

1263.

1264.

1265.

1266.

1267.

1268.

1269.

1270 .
Group III: Array data--Continued

Hydraulic conductivity, in feet per second--Continued

200

300300

200

200

$\begin{array}{llllll}5 & 10 & 20 & 20 & 70 & 70\end{array}$

$\begin{array}{lllllllllllllll} & 2 & 5 & 5 & 5 & 5 & 5 & 5 & 30 & 50 & 40 & 50 & 70 & 70 & 70\end{array}$

$\begin{array}{rrrrrrrrrrrrrrrr}80 & 80 & 80 & 80 & 90 & 90 & 90 & 80 & 80 & 90 & 100 & 100 & 100 & 200 & 200 & 200\end{array}$

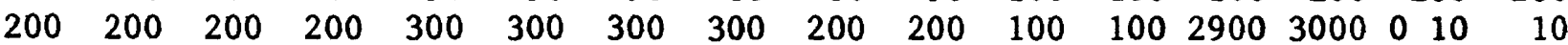

$\begin{array}{llllll}5 & 10 & 10 & 20 & 70 & 70\end{array}$

$\begin{array}{llllllllllllll}5 & 5 & 5 & 5 & 5 & 5 & 10 & 10 & 30 & 20 & 20 & 20 & 100 & 90\end{array}$

$\begin{array}{llllllllllllllll}90 & 90 & 80 & 80 & 80 & 100 & 100 & 100 & 100 & 100 & 100 & 300 & 300 & 300 & 300 & 200\end{array}$

$\begin{array}{llllllllllllllll}200 & 200 & 100 & 100 & 300 & 300 & 200 & 200 & 200 & 200 & 100 & 100 & 3000 & 40 & 5 & 10\end{array}$

$\begin{array}{llllll}30 & 30 & 30 & 50 & 70 & 70\end{array}$

$\begin{array}{llllllllllllll}5 & 2 & 10 & 5 & 10 & 10 & 10 & 10 & 20 & 20 & 20 & 30 & 100 & 100\end{array}$

$\begin{array}{rrrrrrrrrrrrrrrr}80 & 80 & 100 & 100 & 100 & 100 & 100 & 100 & 100 & 100 & 100 & 100 & 300 & 300 & 300 & 300\end{array}$

$\begin{array}{llllllllllllllll}300 & 100 & 100 & 100 & 100 & 100 & 100 & 200 & 200 & 200 & 100 & 100 & 3000 & 40 & 5 & 10\end{array}$

$\begin{array}{llllll}50 & 30 & 40 & 60 & 70 & 70\end{array}$

$\begin{array}{rrrrrrrrrrrrrrr}5 & 10 & 10 & 10 & 10 & 10 & 10 & 10 & 10 & 20 & 20 & 20 & 40 & 90 & 90\end{array}$

$\begin{array}{llllllllllllllll}80 & 80 & 100 & 100 & 100 & 100 & 100 & 70 & 60 & 60 & 40 & 40 & 40 & 60 & 300 & 300\end{array}$

$\begin{array}{llllllllllllllll}300 & 100 & 100 & 100 & 100 & 100 & 100 & 200 & 200 & 200 & 100 & 200 & 2000 & 40 & 5 & 10\end{array}$

$\begin{array}{llllll}50 & 50 & 60 & 60 & 70 & 70\end{array}$

$\begin{array}{lllllllllllllll}10 & 5 & 5 & 5 & 5 & 5 & 5 & 5 & 10 & 20 & 10 & 10 & 30 & 90 & 90\end{array}$

$\begin{array}{llllllllllllllll}80 & 90 & 100 & 100 & 100 & 100 & 100 & 90 & 80 & 50 & 50 & 60 & 60 & 80 & 100 & 400\end{array}$

$\begin{array}{llllllllllllllll}300 & 100 & 100 & 100 & 100 & 100 & 200 & 200 & 300 & 300 & 200 & 400 & 2000 & 40 & 10 & 10\end{array}$

$\begin{array}{llllll}50 & 50 & 60 & 60 & 70 & 70\end{array}$

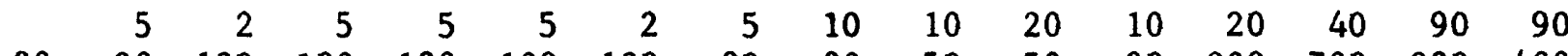

$\begin{array}{llllllllllllllll}90 & 90 & 100 & 100 & 100 & 100 & 100 & 80 & 80 & 50 & 50 & 90 & 200 & 700 & 200 & 400\end{array}$

$\begin{array}{rrrrrrrrrrrrrrrr}300 & 100 & 100 & 100 & 100 & 200 & 200 & 200 & 300 & 200 & 100 & 400 & 1000 & 70 & 10 & 10\end{array}$

$\begin{array}{llllll}30 & 50 & 50 & 60 & 70 & 70\end{array}$

$\begin{array}{lllllllllllllll}5 & 5 & 5 & 5 & 5 & 2 & 5 & 10 & 5 & 20 & 5 & 20 & 40 & 90 & 90\end{array}$

$\begin{array}{llllllllllllllll}90 & 90 & 100 & 100 & 100 & 100 & 100 & 80 & 90 & 60 & 50 & 100 & 200 & 800 & 800 & 400\end{array}$

$\begin{array}{rrrrrrrrrrrrrrrr}500 & 300 & 300 & 200 & 200 & 200 & 200 & 100 & 100 & 100 & 100 & 600 & 900 & 200 & 10 & 1\end{array}$

$\begin{array}{llllll}30 & 30 & 40 & 50 & 70 & 70\end{array}$

$\begin{array}{lllllllllllllll}5 & 5 & 5 & 5 & 2 & 5 & 10 & 20 & 20 & 10 & 5 & 10 & 30 & 40 & 50\end{array}$

$\begin{array}{llllllllllllllll}40 & 60 & 70 & 90 & 90 & 100 & 80 & 80 & 90 & 60 & 60 & 70 & 400 & 700 & 800 & 700\end{array}$

$\begin{array}{rrrrrrrrrrrrrrrr}500 & 500 & 300 & 200 & 200 & 200 & 100 & 100 & 100 & 100 & 100 & 800 & 900 & 200 & 10 & 1\end{array}$

$\begin{array}{llllll}10 & 20 & 40 & 50 & 80 & 80\end{array}$

$\begin{array}{lllllllllllllll}5 & 10 & 10 & 5 & 2 & 5 & 5 & 20 & 20 & 5 & 10 & 10 & 40 & 30 & 30\end{array}$

$\begin{array}{rrrrrrrrrrrrrrrr}40 & 50 & 60 & 60 & 60 & 40 & 40 & 60 & 100 & 100 & 80 & 80 & 100 & 400 & 700 & 700\end{array}$

$\begin{array}{llllllllllllllll}600 & 500 & 300 & 200 & 300 & 100 & 100 & 100 & 100 & 100 & 200 & 800 & 800 & 400 & 10 & 1\end{array}$

$\begin{array}{llllll}30 & 20 & 40 & 50 & 80 & 80\end{array}$

$\begin{array}{lllllllllllllll}5 & 10 & 10 & 20 & 10 & 20 & 20 & 100 & 100 & 5 & 5 & 20 & 30 & 30 & 20\end{array}$

$\begin{array}{llllllllllllllll}10 & 30 & 40 & 30 & 30 & 30 & 90 & 90 & 100 & 200 & 80 & 80 & 80 & 90 & 500 & 500\end{array}$ $\begin{array}{rrrrrrrrrrrrrrrr}600 & 700 & 500 & 500 & 300 & 100 & 100 & 60 & 60 & 70 & 200 & 800 & 800 & 200 & 10 & 1\end{array}$

$30 \quad 10 \quad 50 \quad 50 \quad 50 \quad 50$

$\begin{array}{lllllllllllllll}10 & 10 & 10 & 20 & 20 & 10 & 30 & 100 & 100 & 10 & 5 & 10 & 10 & 10 & 10\end{array}$

$\begin{array}{llllllllllllllll}10 & 10 & 20 & 30 & 50 & 50 & 90 & 90 & 100 & 300 & 100 & 80 & 70 & 80 & 100 & 300\end{array}$ $\begin{array}{rrrrrrrrrrrrrrrr}500 & 700 & 700 & 500 & 300 & 100 & 100 & 60 & 60 & 70 & 100 & 800 & 800 & 200 & 10 & 1\end{array}$

$\begin{array}{llllll}30 & 10 & 70 & 80 & 80 & 80\end{array}$

$\begin{array}{lllllllllllllll}10 & 10 & 20 & 20 & 20 & 20 & 30 & 200 & 200 & 20 & 10 & 10 & 10 & 10 & 10\end{array}$

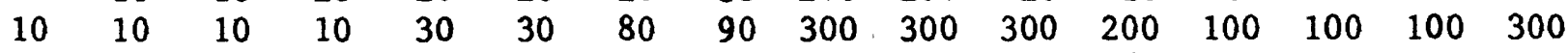

$\begin{array}{rlllllllllllllll}300 & 600 & 700 & 700 & 500 & 500 & 100 & 100 & 60 & 20 & 20 & 60 & 100 & 100 & 10 & 1\end{array}$ 
Table 3.--Listing of data for 1971-77--Continued

Card number

1271

1272.

1273.

1274 .

1275 .

1276.

1277.

1278 .

1279 .

1280 .

1281 .

1282 .

1283.

1284 .

1285 .

1286 .

1287 .

1288 .

1289.

1290 .

1291.

1292.

1293.

1294.

1295.
Group III: Array data--Continued

Hydraulic conductivity, in feet per second--Continued

\begin{tabular}{|c|c|c|c|c|c|c|c|c|c|c|c|c|c|c|c|}
\hline \multirow[t]{2}{*}{30} & 10 & 80 & 100 & 100 & 90 & & & & & & & & & & \\
\hline & 10 & 10 & 20 & 20 & 20 & 30 & 30 & 500 & 200 & 20 & 10 & 10 & 10 & 10 & 10 \\
\hline 10 & 10 & 10 & 10 & 20 & 30 & 80 & 60 & 200 & 300 & 300 & 300 & 100 & 100 & 100 & 300 \\
\hline 500 & 400 & 400 & 700 & 700 & 500 & 200 & 200 & 20 & 20 & 10 & 20 & 30 & 30 & 1 & 1 \\
\hline 30 & 10 & 80 & 100 & 100 & 100 & & & & & & & & & & \\
\hline & 10 & 20 & 20 & 20 & 20 & 30 & 80 & 700 & 700 & 40 & 10 & 5 & 5 & 5 & 5 \\
\hline 30 & 30 & 70 & 70 & 20 & 20 & 50 & 50 & 100 & 100 & 500 & 500 & 300 & 300 & 500 & 500 \\
\hline 800 & 400 & 400 & 400 & 700 & 700 & 50 & 50 & 10 & 10 & 10 & 20 & 30 & 30 & 5 & 10 \\
\hline 10 & 10 & 80 & 100 & 100 & 100 & & & & & & & & & & \\
\hline & 10 & 20 & 30 & 30 & 30 & 30 & 80 & 700 & 700 & 50 & 10 & 7 & 7 & 5 & 5 \\
\hline 30 & 30 & 70 & 70 & 60 & 50 & 60 & 100 & 100 & 100 & 300 & 300 & 300 & 300 & 500 & 700 \\
\hline 800 & 800 & 800 & 400 & 700 & 700 & 100 & 20 & 10 & 10 & 10 & 30 & 30 & 30 & 5 & 10 \\
\hline 10 & 10 & 10 & 80 & 100 & 100 & & & & & & & & & & \\
\hline & 20 & 30 & 30 & 30 & 30 & 30 & 100 & 700 & 700 & 400 & 60 & 50 & 50 & 50 & 50 \\
\hline 100 & 100 & 200 & 200 & 200 & 200 & 100 & 100 & 200 & 300 & 300 & 300 & 600 & 600 & 800 & 800 \\
\hline 800 & 800 & 800 & 800 & 800 & 800 & 800 & 400 & 20 & 10 & 10 & 20 & 10 & 10 & 5 & 5 \\
\hline 20 & 20 & 10 & 80 & 100 & 100 & & & & & & & & & & \\
\hline & 10 & 50 & 50 & 40 & 40 & 70 & 200 & 700 & 700 & 600 & 60 & 50 & 50 & 50 & 50 \\
\hline 90 & 100 & 300 & 300 & 400 & 400 & 300 & 300 & 300 & 300 & 300 & 300 & 800 & 800 & 800 & 800 \\
\hline 800 & 900 & 800 & 800 & 900 & 800 & 800 & 500 & 20 & 20 & 30 & 20 & 10 & 5 & 10 & 10 \\
\hline 20 & 20 & 90 & 90 & 100 & 100 & & & & & & & & & & \\
\hline 0 & & & & & & & & & & & & & & & \\
\hline 0 & & & & & & & & & & & & & & & \\
\hline 0 & & & & & & & & & & & & & & & \\
\hline & & & & & & & & & & & & & & & \\
\hline
\end{tabular}

Group III: Array data--Continued

Bottom of aquifer, in feet

(First card is parameter card)

1296.

1297.

1298.

1299.

1300 .

1301 .

1302 .

1303.

1304.

1305.

1306.

1307.

1308.

1309 .

1310 .

1311.

1312.

1313.

12

0

0

0

0

$50905050 \quad 5010 \quad 5000 \quad 4980 \quad 4950 \quad 4930 \quad 4910 \quad 4900 \quad 4860 \quad 4840 \quad 4820 \quad 4810 \quad 4800 \quad 4770 \quad 4740$ 4720470046804660

0

$5080 \quad 5040 \quad 5000 \quad 4990 \quad 4950 \quad 49204910 \quad 4900 \quad 4890 \quad 4840 \quad 4820 \quad 4800 \quad 4790 \quad 4780 \quad 4740 \quad 4720$ 4700468046604640

0

$\begin{array}{llllllll}5500 & 5450 & 5380 & 5290 & 5210 & 5190 & 5130 & 5100\end{array}$

$\begin{array}{llllllllllllllllll}5060 & 5020 & 4990 & 4970 & 4940 & 4900 & 4890 & 4870 & 4850 & 4820 & 4800 & 4780 & 4740 & 4720 & 4700 & 4680\end{array}$

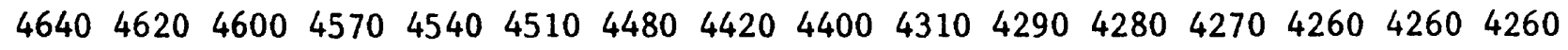
427042704280428042904300

55005440535052805210518051205090 
Table 3.--Listing of data for 1971-77--Continued

Card

number

1314. 1315 . 1316 . 1317. 1318 . 1319 . 1320 . 1321. 1322. 1323. 1324. 1325. 1326. 1327. 1328 . 1329 . 1330 . 1331. 1332. 1333. 1334. 1335. 1336. 1337. 1338 . 1339 . 1340 . 1341. 1342. 1343. 1344. 1345 . 1346. 1347. 1348 . 1349 . 1350 . 1351. 1352. 1353. 1354. 1355. 1356. 1357. 1358 . 1359. 1360 . 1361. 1362.
Group III: Array data--Continued

Bottom of aquifer, in feet--Continued

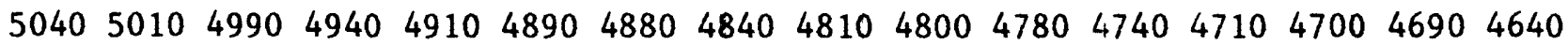
$\begin{array}{lllllllllllllllll}4610 & 4600 & 4550 & 4520 & 4500 & 4480 & 4420 & 4390 & 4320 & 4290 & 4280 & 4280 & 4270 & 4270 & 4270 & 4280\end{array}$ 428042804290430043104310

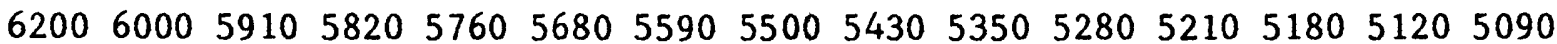
$\begin{array}{llllllllllllllllllll}5040 & 5000 & 4980 & 4940 & 4900 & 4880 & 4850 & 4820 & 4800 & 4780 & 4750 & 4720 & 4700 & 4770 & 4740 & 4710\end{array}$ $4700 \quad 4670 \quad 4730 \quad 4700 \quad 4680 \quad 4640 \quad 4600 \quad 4370 \quad 4320 \quad 4290 \quad 4290 \quad 4280 \quad 4280 \quad 4280 \quad 4280 \quad 4290$ $429043004300 \quad 431043304340$

$\begin{array}{lllllllllllllll}6210 & 6010 & 5920 & 5830 & 5770 & 5680 & 5590 & 5500 & 5430 & 5350 & 5280 & 5200 & 5170 & 5120 & 5090\end{array}$ $5040 \quad 5000 \quad 4970 \quad 4940 \quad 4900 \quad 4870 \quad 4840 \quad 4810 \quad 4790 \quad 4750 \quad 4730 \quad 4710 \quad 4690 \quad 4660 \quad 4630 \quad 4600$ $4570 \quad 4540 \quad 4510 \quad 4490 \quad 4460 \quad 4430 \quad 4400 \quad 4360 \quad 4330 \quad 4300 \quad 4290 \quad 4280 \quad 4280 \quad 4290 \quad 4290 \quad 4300$ $431043104330 \quad 4350 \quad 4350 \quad 4350$

$\begin{array}{lllllllllllllll}6280 & 6080 & 5960 & 5880 & 5780 & 5690 & 5590 & 5500 & 5430 & 5350 & 5280 & 5200 & 5170 & 5120 & 5090\end{array}$ $\begin{array}{lllllllllllllllll}5040 & 5000 & 4960 & 4930 & 4900 & 4870 & 4830 & 4800 & 4790 & 4750 & 4730 & 4700 & 4670 & 4640 & 4610 & 4590\end{array}$ $4560 \quad 4540 \quad 4510 \quad 4500 \quad 4460 \quad 4430 \quad 4410 \quad 4390 \quad 4360 \quad 4330 \quad 4300 \quad 4290 \quad 4290 \quad 4300 \quad 4310 \quad 4320$

$43504370 \quad 4390 \quad 4390 \quad 4370 \quad 4350$ $\begin{array}{llllllllllllllll}6300 & 6100 & 5980 & 5890 & 5790 & 5690 & 5590 & 5500 & 5430 & 5350 & 5270 & 5200 & 5170 & 5120 & 5090\end{array}$ $5040 \quad 5000 \quad 4970 \quad 4940 \quad 4900 \quad 4860 \quad 4830 \quad 4800 \quad 4770 \quad 4740 \quad 4710 \quad 4690 \quad 4670 \quad 4640 \quad 4600 \quad 4580$ $\begin{array}{lllllllllllllllll}4550 & 4540 \quad 4520 & 4500 & 4470 & 4440 & 4420 & 4400 & 4390 & 4360 & 4330 & 4310 & 4310 & 4320 & 4340 & 4360\end{array}$ 439044004410441043904360 $\begin{array}{llllllllllllllll}6380 & 6180 & 6000 & 5900 & 5790 & 5690 & 5590 & 5500 & 5430 & 5350 & 5270 & 5210 & 5170 & 5120 & 5090\end{array}$ $5040 \quad 5010 \quad 4980 \quad 4940 \quad 4900 \quad 4870 \quad 4840 \quad 4800 \quad 4790 \quad 4760 \quad 4730 \quad 4700 \quad 4670 \quad 4640 \quad 4610 \quad 4590$ $4580 \quad 4540 \quad 4520 \quad 4500 \quad 4480 \quad 4460 \quad 4440 \quad 4420 \quad 4400 \quad 4390 \quad 4370 \quad 4350 \quad 4340 \quad 4350 \quad 4380 \quad 4390$ 441044204430442044004360 $\begin{array}{llllllllllllllll}6400 & 620 & 6010 & 5910 & 5790 & 5700 & 5590 & 5510 & 5420 & 5350 & 5280 & 5200 & 5170 & 5120 & 5090\end{array}$ $\begin{array}{llllllllllllllllll}5050 & 5010 & 4990 & 4950 & 4900 & 4880 & 4850 & 4810 & 4790 & 4770 & 4730 & 4700 & 4690 & 4670 & 4630 & 4600\end{array}$ $4590 \quad 4570 \quad 4540 \quad 4510 \quad 4490 \quad 4480 \quad 4460 \quad 4440 \quad 4420 \quad 4410 \quad 4390 \quad 4390 \quad 4380 \quad 4390 \quad 4400 \quad 4410$ 442044404460444044104360

$\begin{array}{llllllllllllllll}6420 & 6250 & 6070 & 5910 & 5800 & 5700 & 5600 & 5510 & 5430 & 5350 & 5280 & 5210 & 5190 & 5140 & 5100\end{array}$ $\begin{array}{llllllllllllllllll}5170 & 5130 & 5000 & 4970 & 4920 & 4900 & 4860 & 4830 & 4800 & 4770 & 4740 & 4720 & 4700 & 4690 & 4660 & 4630\end{array}$ $4600 \quad 4590 \quad 4570 \quad 4540 \quad 4510 \quad 4490 \quad 4480 \quad 4450 \quad 4440 \quad 4430 \quad 4410 \quad 4410 \quad 4400 \quad 4400 \quad 4410 \quad 4430$ 446044904490446044104370

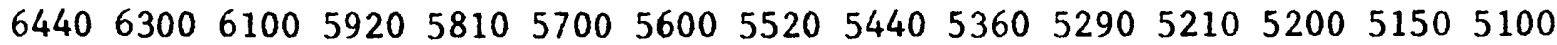
$\begin{array}{lllllllllllllllll}5080 & 5040 & 5010 & 4980 & 4950 & 4910 & 4890 & 4850 & 4810 & 4790 & 4770 & 4750 & 4720 & 4700 & 4690 & 4660\end{array}$ $4640 \quad 4620 \quad 4600 \quad 4570 \quad 4540 \quad 4510 \quad 4490 \quad 4480 \quad 4460 \quad 4440 \quad 4430 \quad 4420 \quad 4420 \quad 4440 \quad 4460 \quad 4490$ $45004510 \quad 45104490 \quad 44204480$

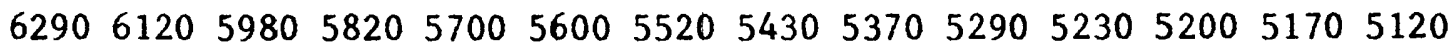
$\begin{array}{lllllllllllllllllll}5100 & 5070 & 5040 & 5000 & 4970 & 4940 & 4910 & 4880 & 4850 & 4820 & 4800 & 4780 & 4750 & 4730 & 4720 & 4700\end{array}$ $\begin{array}{llllllllllllllllll}4680 & 4650 & 4620 & 4600 & 4580 & 4550 & 4510 & 4500 & 4490 & 4480 & 4480 & 4480 & 4480 & 4490 & 4500 & 4510\end{array}$ $45304550 \quad 4530 \quad 4500 \quad 44304480$

$\begin{array}{llllllllllllll}6330 & 6150 & 5970 & 5810 & 5710 & 5600 & 5520 & 5450 & 5380 & 5290 & 5240 & 5200 & 5190 & 5150\end{array}$ $\begin{array}{llllllllllllllllll}5110 & 5090 & 5060 & 5030 & 5000 & 4970 & 4930 & 4900 & 4880 & 4860 & 4830 & 4800 & 4780 & 4760 & 4740 & 4720\end{array}$ $4700 \quad 4680 \quad 4660 \quad 4640 \quad 4610 \quad 4590 \quad 4550 \quad 4530 \quad 4520 \quad 4500 \quad 4500 \quad 4500 \quad 4500 \quad 4510 \quad 4520 \quad 4540$ $456045804580 \quad 453044504390$

$\begin{array}{lllllllllllllll}6430 & 6350 & 6150 & 5980 & 5840 & 5720 & 5600 & 5520 & 5460 & 5380 & 5300 & 5260 & 5230 & 5200 & 5170\end{array}$ $\begin{array}{lllllllllllllllll}5140 & 5110 & 5080 & 5050 & 5020 & 5000 & 4970 & 4940 & 4910 & 4880 & 4860 & 4840 & 4820 & 4800 & 4780 & 4760\end{array}$ $4740 \quad 4720 \quad 4690 \quad 4660 \quad 4630 \quad 4610 \quad 4590 \quad 4570 \quad 4550 \quad 4530 \quad 4520 \quad 4520 \quad 4540 \quad 4560 \quad 4580 \quad 4600$ $46104610 \quad 46104570 \quad 4480 \quad 4390$

$\begin{array}{lllllllllllllll}6430 & 6400 & 620 & 6000 & 5830 & 5720 & 5610 & 5530 & 5470 & 5380 & 5310 & 5270 & 5230 & 5200 & 5180\end{array}$ $\begin{array}{llllllllllllllll}5160 & 5130 & 5100 & 5080 & 5050 & 5020 & 5000 & 4980 & 4950 & 4920 & 4900 & 4870 & 4850 & 4830 & 4800 & 4780\end{array}$ 
Table 3.--Listing of data for 1971-77--Continued

Card

number

1363.

1364 .

1365 .

1366.

1367.

1368.

1369 .

1370 .

1371.

1372 .

1373.

1374.

1375 .

1376.

1377.

1378 .

1379 .

1380 .

1381 .

1382.

1383.

1384 .

1385 .

1386 .

1387.

1388 .

1389 .

1390 .

1391.

1392.

1393.

1394.

1395.

1396.

1397.

1398.

1399.

1400 .

1401 .

1402 .

1403.

1404.

1405 .

1406 .

1407 .

1408 .

1409 .

1410 .

1411.
Group III: Array data--Continued

Bottom of aquifer, in feet--Continued
$4760 \quad 4740 \quad 4720 \quad 4700 \quad 4680 \quad 4660 \quad 4640 \quad 4610 \quad 4590 \quad 4580 \quad 4580 \quad 4580 \quad 4590 \quad 4600 \quad 4630 \quad 4660$ $468046804640 \quad 4590 \quad 4490 \quad 4400$

$\begin{array}{lllllllllllllll}6510 & 6420 & 620 & 6000 & 5830 & 5720 & 5610 & 5530 & 5480 & 5400 & 5330 & 5290 & 5250 & 5210 & 5190\end{array}$ $\begin{array}{llllllllllllllllll}5170 & 5140 & 5120 & 5100 & 5080 & 5050 & 5020 & 5000 & 4970 & 4940 & 4910 & 4900 & 4880 & 4860 & 4840 & 4810\end{array}$ $4790 \quad 4770 \quad 4750 \quad 4730 \quad 4700 \quad 4680 \quad 4660 \quad 4640 \quad 4620 \quad 4610 \quad 4610 \quad 4610 \quad 4620 \quad 4640 \quad 4680 \quad 4700$ $4710 \quad 471046804630 \quad 4500 \quad 4400$

$\begin{array}{lllllllllllllll}6535 & 6450 & 6220 & 6000 & 5850 & 5730 & 5620 & 5550 & 5470 & 5400 & 5340 & 5300 & 5270 & 5240 & 5210\end{array}$ $\begin{array}{lllllllllllllllll}5180 & 5160 & 5140 & 5120 & 5100 & 5070 & 5040 & 5020 & 5000 & 4980 & 4960 & 4940 & 4920 & 4900 & 4870 & 4840\end{array}$ $4820 \quad 4800 \quad 4780 \quad 4760 \quad 4740 \quad 4720 \quad 4700 \quad 4680 \quad 4670 \quad 4660 \quad 4670 \quad 4680 \quad 4690 \quad 4700 \quad 4700 \quad 4710$ $4710471047004630 \quad 45204410$

$\begin{array}{lllllllllllllll}6570 & 6450 & 6220 & 6000 & 5850 & 5730 & 5620 & 5560 & 5480 & 5410 & 5360 & 5310 & 5290 & 5260 & 5230\end{array}$ $\begin{array}{lllllllllllllllll}5200 & 5180 & 5160 & 5140 & 5120 & 5100 & 5080 & 5060 & 5030 & 5010 & 4980 & 4950 & 4930 & 4920 & 4900 & 4880\end{array}$ $48604830 \quad 4810 \quad 4790 \quad 4770 \quad 4750 \quad 4730 \quad 4720 \quad 4710 \quad 4710 \quad 4710 \quad 4710 \quad 4720 \quad 4720 \quad 4720 \quad 4720$ $4720471047004640 \quad 45204410$

$\begin{array}{llllllllllllllll}6600 & 6500 & 6230 & 6010 & 5850 & 5750 & 5650 & 5570 & 5490 & 5420 & 5350 & 5320 & 5300 & 5280 & 5250\end{array}$

$\begin{array}{lllllllllllllllll}5220 & 5200 & 5180 & 5160 & 5140 & 5120 & 5100 & 5080 & 5060 & 5040 & 5010 & 4990 & 4970 & 4950 & 4930 & 4900\end{array}$ $4880 \quad 4860 \quad 4840 \quad 4820 \quad 4800 \quad 4780 \quad 4750 \quad 4730 \quad 4730 \quad 4720 \quad 4720 \quad 4720 \quad 4720 \quad 4720 \quad 4720 \quad 4720$ $4820 \quad 471047004640 \quad 4530 \quad 4420$

$\begin{array}{lllllllllllllll}6630 & 6520 & 6250 & 6010 & 5870 & 5750 & 5650 & 5580 & 5500 & 5420 & 5380 & 5330 & 5310 & 5290 & 5260\end{array}$ $\begin{array}{llllllllllllllllll}5240 & 5210 & 5200 & 5180 & 5160 & 5140 & 5120 & 5100 & 5080 & 5060 & 5030 & 5000 & 4990 & 4970 & 4940 & 4920\end{array}$ $4900 \quad 4880 \quad 4860 \quad 4840 \quad 4820 \quad 4800 \quad 4780 \quad 4770 \quad 4750 \quad 4740 \quad 4730 \quad 4730 \quad 4730 \quad 4730 \quad 4730 \quad 4720$ $47204710470046404540 \quad 4430$

$\begin{array}{lllllllllllllll}6650 & 6540 & 6280 & 6010 & 5870 & 5750 & 5660 & 5580 & 5500 & 5430 & 5380 & 5340 & 5320 & 5300 & 5280\end{array}$ $\begin{array}{lllllllllllllllll}5260 & 5240 & 5220 & 5200 & 5180 & 5160 & 5140 & 5120 & 5100 & 5080 & 5060 & 5030 & 5000 & 4980 & 4960 & 4940\end{array}$ $4920 \quad 4900 \quad 4880 \quad 4860 \quad 4830 \quad 4800 \quad 4790 \quad 4780 \quad 4760 \quad 4740 \quad 4730 \quad 4730 \quad 4730 \quad 4730 \quad 4730 \quad 4720$ 472047104700462045304420

$\begin{array}{lllllllllllllll}6630 & 6530 & 6280 & 6000 & 5870 & 5740 & 5670 & 5580 & 5510 & 5440 & 5390 & 5360 & 5330 & 5310 & 5300\end{array}$ $\begin{array}{lllllllllllllllll}5280 & 5260 & 5240 & 5220 & 5190 & 5170 & 5150 & 5130 & 5110 & 5090 & 5070 & 5050 & 5030 & 5000 & 4970 & 4950\end{array}$ $4920 \quad 4900 \quad 4880 \quad 4860 \quad 4840 \quad 4820 \quad 4790 \quad 4760 \quad 4730 \quad 4730 \quad 4730 \quad 4720 \quad 4720 \quad 4720 \quad 4720 \quad 4710$ $47104710468046104530 \quad 4410$

$\begin{array}{lllllllllllllll}6600 & 6530 & 6290 & 6000 & 5860 & 5740 & 5670 & 5580 & 5510 & 5440 & 5400 & 5370 & 5340 & 5320 & 5310\end{array}$ $\begin{array}{lllllllllllllllll}5290 & 5270 & 5250 & 5230 & 5210 & 5190 & 5170 & 5150 & 5130 & 5110 & 5090 & 5070 & 5040 & 5010 & 4990 & 4970\end{array}$ $4940 \quad 4910 \quad 4880 \quad 4860 \quad 4840 \quad 4810 \quad 4780 \quad 4750 \quad 4720 \quad 4710 \quad 4700 \quad 4700 \quad 4700 \quad 4700 \quad 4700 \quad 4700$ $470046804640 \quad 459045204410$

$\begin{array}{lllllllllllllll}6560 & 6520 & 6300 & 6000 & 5850 & 5740 & 5670 & 5590 & 5520 & 5450 & 5410 & 5380 & 5360 & 5340 & 5320\end{array}$ $\begin{array}{llllllllllllllllll}5300 & 5280 & 5260 & 5240 & 5220 & 5200 & 5180 & 5160 & 5140 & 5120 & 5100 & 5070 & 5040 & 5010 & 4990 & 4960\end{array}$ $\begin{array}{llllllllllllllll}4930 & 4910 & 4880 & 4860 & 4840 & 4800 & 4770 & 4730 & 4690 & 4680 & 4680 & 4670 & 4660 & 4650 & 4630 & 4610\end{array}$ 460046004580454045004430

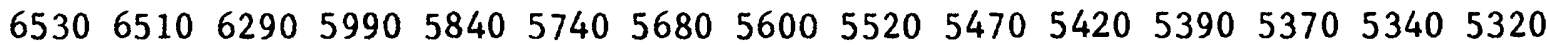
$\begin{array}{llllllllllllllllll}5310 & 5290 & 5270 & 5250 & 5230 & 5200 & 5180 & 5160 & 5140 & 5120 & 5100 & 5070 & 5040 & 5020 & 5000 & 4970\end{array}$ $4940 \quad 4910 \quad 4880 \quad 4860 \quad 4830 \quad 4800 \quad 4770 \quad 4730 \quad 4700 \quad 4690 \quad 4680 \quad 4660 \quad 4640 \quad 4620 \quad 4600 \quad 4590$ $45804580 \quad 4570 \quad 4560 \quad 4480 \quad 4450$

$\begin{array}{llllllllllllll}6490 & 6290 & 5980 & 5820 & 5740 & 5680 & 5600 & 5520 & 5470 & 5430 & 5390 & 5380 & 5360 & 5340\end{array}$ $\begin{array}{llllllllllllllll}5320 & 5300 & 5280 & 5260 & 5240 & 5220 & 5190 & 5170 & 5150 & 5130 & 5100 & 5080 & 5050 & 5020 & 5000 & 4970\end{array}$

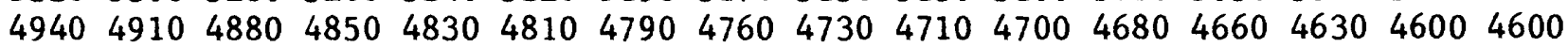
459045604530450044704450

$\begin{array}{lllllllllllllll}6490 & 6290 & 5970 & 5810 & 5750 & 5690 & 5600 & 5520 & 5470 & 5430 & 5400 & 5380 & 5360 & 5340\end{array}$ $\begin{array}{llllllllllllllll}5330 & 5310 & 5290 & 5270 & 5250 & 5220 & 5200 & 5180 & 5150 & 5120 & 5100 & 5080 & 5050 & 5020 & 5000 & 4970\end{array}$ $4940 \quad 4910 \quad 4890 \quad 4870 \quad 4850 \quad 4830 \quad 4810 \quad 4790 \quad 4770 \quad 4740 \quad 4720 \quad 4700 \quad 4690 \quad 4670 \quad 4640 \quad 4620$ 
Table 3.--Listing of data for 1971-77--Continued

Card

number

1412.

1413.

1414.

1415.

1416.

1417.

1418 .

1419 .

1420 .

1421.

1422 .

1423.

1424.

1425 .

1426.

1427.

1428 .

1429 .

1430 .

1431.

1432 .

1433.

1434 .

1435 .

1436.

1437.

1438.

1439.

1440 .

1441.

1442.

1443.

1444 .

1445 .

1446 .

1447.

1448 .

1449 .

1450 .

1451 .

1452 .

1453.

1454 .

1455 .

1456 .

1457.

1458 .

1459 .

1460 .
Group III: Array data--Continued

Bottom of aquifer, in feet--Continued $\begin{array}{rrrrrrrrrrrrr}4600 & 4590 & 4560 & 4500 & 4480 & 4450 & & & & & & & \\ 6280 & 5920 & 5800 & 5740 & 5700 & 5610 & 5520 & 5480 & 5440 & 5410 & 5390 & 5370 & 5350\end{array}$

$\begin{array}{lllllllllllllllll}5330 & 5320 & 5300 & 5280 & 5250 & 5220 & 5200 & 5170 & 5140 & 5120 & 5100 & 5080 & 5050 & 5020 & 5000 & 4970\end{array}$

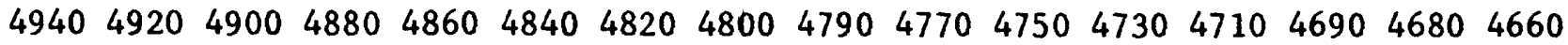
464046204600455044904470

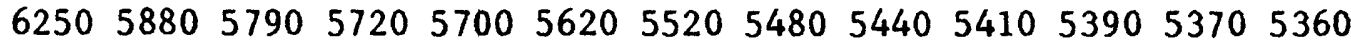

$\begin{array}{lllllllllllllllll}5340 & 5320 & 5310 & 5290 & 5270 & 5240 & 5200 & 5180 & 5150 & 5120 & 5100 & 5080 & 5060 & 5030 & 5000 & 4970\end{array}$ $4940 \quad 4920 \quad 4900 \quad 4880 \quad 4860 \quad 4840 \quad 4830 \quad 4820 \quad 48104800 \quad 4780 \quad 4760 \quad 4740 \quad 4720 \quad 4700 \quad 4690$ 468046604630459045104480

$\begin{array}{lllllllllllll}6180 & 5880 & 5770 & 5700 & 5700 & 5620 & 5520 & 5480 & 5440 & 5420 & 5400 & 5380 & 5370\end{array}$

$\begin{array}{llllllllllllllll}5350 & 5330 & 5310 & 5300 & 5270 & 5240 & 5210 & 5180 & 5150 & 5120 & 5100 & 5070 & 5040 & 5020 & 5000 & 4980\end{array}$

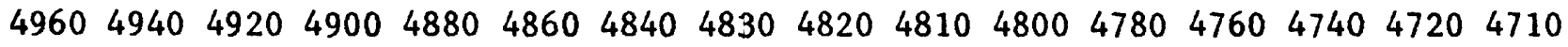
$4700468046504610 \quad 45604490$

$\begin{array}{lllllllllllll}6150 & 5850 & 5740 & 5700 & 5700 & 5620 & 5530 & 5480 & 5440 & 5430 & 5410 & 5390 & 5380\end{array}$

$\begin{array}{llllllllllllllll}5360 & 5340 & 5320 & 5300 & 5280 & 5260 & 5220 & 5190 & 5150 & 5110 & 5090 & 5070 & 5040 & 5020 & 5000 & 4980\end{array}$

$\begin{array}{llllllllllllllllll}4960 & 4940 & 4920 & 4900 & 4890 & 4880 & 4860 & 4840 & 4830 & 4820 & 4810 & 4800 & 4790 & 4770 & 4750 & 4730\end{array}$

471046904670464045904510

$\begin{array}{lllllllllllllll}6500 & 6100 & 5830 & 5730 & 5700 & 5700 & 5630 & 5520 & 5480 & 5450 & 5430 & 5410 & 5390 & 5370\end{array}$

$\begin{array}{llllllllllllllllll}5350 & 5340 & 5320 & 5300 & 5280 & 5250 & 5220 & 5190 & 5160 & 5120 & 5090 & 5060 & 5040 & 5020 & 4990 & 4970\end{array}$

$\begin{array}{lllllllllllllllllll}4950 & 4940 & 4930 & 4920 & 4900 & 4880 & 4870 & 4860 & 4850 & 4840 & 4830 & 4820 & 4800 & 4780 & 4760 & 4740\end{array}$

472047004680465046004540

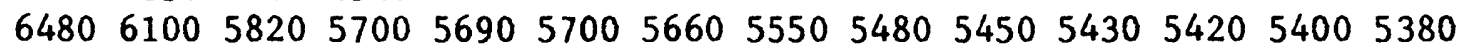

$\begin{array}{lllllllllllllllll}5360 & 5340 & 5330 & 5310 & 5290 & 5270 & 5240 & 5200 & 5160 & 5120 & 5090 & 5070 & 5040 & 5020 & 4990 & 4980\end{array}$

$\begin{array}{lllllllllllllllllll}4960 & 4950 & 4940 & 4930 & 4920 & 4900 & 4890 & 4880 & 4870 & 4850 & 4840 & 4830 & 4810 & 4800 & 4790 & 4770\end{array}$

475047304700467046304580

$\begin{array}{lllllllllllllll}6450 & 6080 & 5810 & 5700 & 5680 & 5700 & 5680 & 5580 & 5490 & 5460 & 5440 & 5420 & 5400 & 5390\end{array}$

$\begin{array}{lllllllllllllllll}5370 & 5350 & 5330 & 5320 & 5300 & 5270 & 5240 & 5200 & 5170 & 5140 & 5100 & 5070 & 5040 & 5010 & 4990 & 4980\end{array}$ $4970 \quad 4960 \quad 4950 \quad 4940 \quad 4930 \quad 4920 \quad 4910 \quad 4900 \quad 4880 \quad 4870 \quad 4850 \quad 4840 \quad 4830 \quad 4820 \quad 4800 \quad 4790$ 477047504720468046404600

$\begin{array}{lllllllllllllll}6450 & 6080 & 5810 & 5700 & 5680 & 5700 & 5690 & 5590 & 5490 & 5470 & 5450 & 5430 & 5410 & 5390\end{array}$

$\begin{array}{lllllllllllllllll}5380 & 5370 & 5350 & 5330 & 5300 & 5270 & 5240 & 5200 & 5170 & 5140 & 5100 & 5080 & 5060 & 5030 & 5000 & 4990\end{array}$ $4980 \quad 4970 \quad 4960 \quad 4950 \quad 4940 \quad 4930 \quad 4920 \quad 4910 \quad 4890 \quad 4880 \quad 4870 \quad 4860 \quad 4840 \quad 4830 \quad 4820 \quad 4800$ 479047704740470046604610

$\begin{array}{llllllllllllllll}6900 & 6400 & 6080 & 5820 & 5700 & 5680 & 5690 & 5700 & 5600 & 5500 & 5470 & 5450 & 5440 & 5420 & 5400\end{array}$

$\begin{array}{lllllllllllllllll}5380 & 5370 & 5350 & 5330 & 5300 & 5280 & 5240 & 5200 & 5180 & 5140 & 5100 & 5090 & 5070 & 5040 & 5020 & 5000\end{array}$ $4990 \quad 4980 \quad 4970 \quad 4960 \quad 4950 \quad 4940 \quad 4930 \quad 4920 \quad 4900 \quad 4890 \quad 4880 \quad 4870 \quad 4850 \quad 4830 \quad 4820 \quad 4810$ $480047804740 \quad 471046804620$

$\begin{array}{lllllllllllllllll}6900 & 6420 & 6090 & 5880 & 5750 & 5690 & 5690 & 5700 & 5630 & 5510 & 5480 & 5450 & 5440 & 5420 & 5400\end{array}$ $\begin{array}{llllllllllllllllll}5390 & 5380 & 5360 & 5330 & 5310 & 5290 & 5250 & 5210 & 5180 & 5150 & 5120 & 5100 & 5080 & 5050 & 5030 & 5010\end{array}$

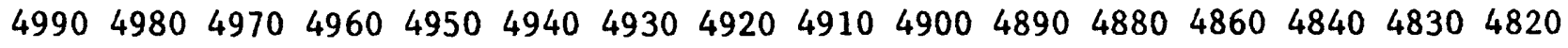
480047804750472046804630

$\begin{array}{llllllllllllllll}6900 & 6420 & 6090 & 5880 & 5720 & 5690 & 5690 & 5700 & 5630 & 5520 & 5480 & 5460 & 5440 & 5430 & 5410\end{array}$ $\begin{array}{llllllllllllllll}5390 & 5380 & 5350 & 5330 & 5310 & 5290 & 5270 & 5240 & 5190 & 5170 & 5140 & 5100 & 5090 & 5070 & 5050 & 5030\end{array}$

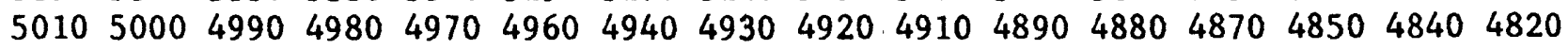
480047804760472046804630

$\begin{array}{lllllllllllllllll}6850 & 6420 & 6110 & 5900 & 5780 & 5700 & 5690 & 5700 & 5660 & 5560 & 5490 & 5470 & 5450 & 5430 & 5410\end{array}$ $\begin{array}{llllllllllllllll}5390 & 5380 & 5360 & 5340 & 5310 & 5280 & 5250 & 5220 & 5190 & 5160 & 5130 & 5110 & 5100 & 5080 & 5060 & 5040\end{array}$

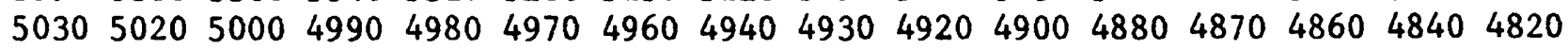
$480047804750 \quad 472046804640$ 
Table 3.--Listing of data for 1971-77--Continued

Card

number

1461 .

1462.

1463.

1464.

1465.

1466.

1467.

1468.

1469 .

1470 .

1471.

1472 .

1473.

1474 .

1475 .

1476.

1477 .

1478 .

1479 .

1480 .

1481 .

1482 .

1483.

1484.

1485 .

1486.

1487.

1488 .

1489 .

1490 .

1491.

1492.

1493.

1494.

1495.

1496.

1497.

1498 .

1499.

1500 .

1501 .

1502.

1503.

1504.
Group III: Array data--Continued

Bottom of aquifer, in feet--Continued

\begin{abstract}
$\begin{array}{lllllllllllllll}6850 & 6400 & 6120 & 6040 & 5820 & 5720 & 5690 & 5700 & 5690 & 5590 & 5500 & 5470 & 5460 & 5440 & 5420\end{array}$ $\begin{array}{llllllllllllllll}5400 & 5380 & 5360 & 5340 & 5320 & 5290 & 5260 & 5230 & 5200 & 5180 & 5160 & 5130 & 5110 & 5090 & 5070 & 5050\end{array}$ $\begin{array}{llllllllllllllll}5040 & 5030 & 5020 & 5000 & 4990 & 4980 & 4970 & 4960 & 4940 & 4920 & 4900 & 4890 & 4870 & 4850 & 4830 & 4820\end{array}$ 480047804760473046804640 $\begin{array}{llllllllllllllll}6850 & 6400 & 6150 & 5980 & 5880 & 5780 & 5700 & 5700 & 5700 & 5610 & 5520 & 5480 & 5460 & 5440 & 5420\end{array}$ $\begin{array}{lllllllllllllllll}5400 & 5390 & 5370 & 5350 & 5320 & 5290 & 5260 & 5230 & 5200 & 5180 & 5160 & 5140 & 5120 & 5100 & 5090 & 5070\end{array}$ $\begin{array}{lllllllllllllllll}5050 & 5040 & 5020 & 5010 & 5000 & 4980 & 4960 & 4940 & 4930 & 4920 & 4910 & 4890 & 4880 & 4860 & 4840 & 4820\end{array}$ 480047804760472046804640
\end{abstract}

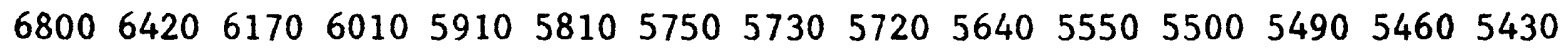
$\begin{array}{llllllllllllllll}5410 & 5390 & 5370 & 5350 & 5320 & 5300 & 5270 & 5240 & 5210 & 5190 & 5170 & 5150 & 5130 & 5110 & 5090 & 5080\end{array}$ $\begin{array}{lllllllllllllllll}5060 & 5050 & 5030 & 5020 & 5000 & 4990 & 4970 & 4950 & 4940 & 4930 & 4910 & 4890 & 4880 & 4860 & 4830 & 4810\end{array}$ $479047704740 \quad 471046804640$

$\begin{array}{llllllllllllllll}6770 & 6440 & 6180 & 6050 & 5930 & 5860 & 5790 & 5770 & 5730 & 5630 & 5550 & 5500 & 5490 & 5460 & 5440\end{array}$ $\begin{array}{lllllllllllllllll}5410 & 5390 & 5370 & 5350 & 5330 & 5300 & 5280 & 5260 & 5230 & 5200 & 5180 & 5160 & 5140 & 5120 & 5100 & 5080\end{array}$ $\begin{array}{llllllllllllllll}5070 & 5060 & 5040 & 5030 & 5010 & 4990 & 4970 & 4960 & 4940 & 4930 & 4910 & 4890 & 4870 & 4850 & 4830 & 4810\end{array}$ $47904780 \quad 4740 \quad 4710 \quad 4680 \quad 4640$ $\begin{array}{lllllllllllllllll}6680 & 6450 & 6200 & 6050 & 5930 & 5860 & 5780 & 5720 & 5750 & 5700 & 5600 & 5540 & 5500 & 5490 & 5470\end{array}$ $\begin{array}{llllllllllllllllll}5440 & 5400 & 5380 & 5360 & 5340 & 5310 & 5280 & 5250 & 5230 & 5200 & 5180 & 5170 & 5150 & 5130 & 5110 & 5090\end{array}$ $\begin{array}{llllllllllllllllll}5070 & 5060 & 5040 & 5030 & 5010 & 4990 & 4980 & 4970 & 4950 & 4930 & 4910 & 4890 & 4880 & 4860 & 4840 & 4810\end{array}$ $479047704730 \quad 470046804640$ $\begin{array}{llllllllllllllll}6600 & 6420 & 6220 & 6050 & 5930 & 5830 & 5750 & 5650 & 5700 & 5710 & 5610 & 5570 & 5530 & 5500 & 5480\end{array}$

$\begin{array}{lllllllllllllllll}5450 & 5410 & 5390 & 5370 & 5340 & 5310 & 5290 & 5270 & 5240 & 5210 & 5190 & 5170 & 5150 & 5130 & 5120 & 5100\end{array}$ $\begin{array}{lllllllllllllllll}5080 & 5060 & 5040 & 5030 & 5010 & 4990 & 4980 & 4960 & 4940 & 4930 & 4910 & 4890 & 4870 & 4850 & 4830 & 4800\end{array}$ $47804760 \quad 4730 \quad 470046704630$ $\begin{array}{lllllllllllllll}6530 & 6350 & 6180 & 6000 & 5850 & 5750 & 5700 & 5630 & 5640 & 5660 & 5680 & 5590 & 5550 & 5510 & 5490\end{array}$ $\begin{array}{lllllllllllllllllll}5440 & 5410 & 5390 & 5370 & 5340 & 5300 & 5290 & 5250 & 5230 & 5200 & 5190 & 5140 & 5150 & 5130 & 5120 & 5100\end{array}$ $\begin{array}{lllllllllllllllll}5080 & 5060 & 5040 & 5030 & 5010 & 4990 & 4980 & 4970 & 4950 & 4930 & 4910 & 4890 & 4870 & 4850 & 4830 & 4800\end{array}$ 478047604730470046704630 $\begin{array}{lllllllllllllll}6450 & 6280 & 6120 & 5970 & 5840 & 5740 & 5650 & 5610 & 5590 & 5590 & 5620 & 5610 & 5580 & 5530 & 5500\end{array}$ $\begin{array}{lllllllllllllllll}5430 & 5390 & 5340 & 5320 & 5300 & 5280 & 5270 & 5250 & 5220 & 5190 & 5180 & 5140 & 5140 & 5120 & 5100 & 5080\end{array}$ $\begin{array}{llllllllllllllll}5080 & 5070 & 5050 & 5030 & 5010 & 4990 & 4980 & 4970 & 4950 & 4930 & 4910 & 4890 & 4870 & 4840 & 4810 & 4790\end{array}$ $47704740 \quad 4720469046604630$ $\begin{array}{lllllllllllllll}6370 & 6180 & 6050 & 5880 & 5780 & 5680 & 5620 & 5575 & 5560 & 5550 & 5550 & 5550 & 5530 & 5480 & 5430\end{array}$ $\begin{array}{lllllllllllllllll}5380 & 5330 & 5310 & 5280 & 5270 & 5240 & 5220 & 5200 & 5190 & 5160 & 5150 & 5130 & 5110 & 5090 & 5070 & 5060\end{array}$

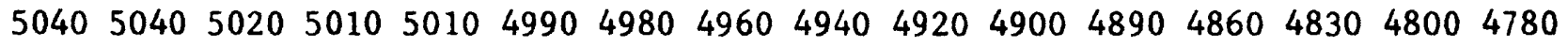
$47604740 \quad 4720468046504620$

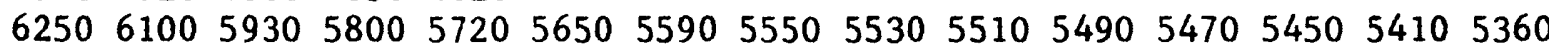
$\begin{array}{lllllllllllllllll}5330 & 5300 & 5270 & 5250 & 5220 & 5200 & 5190 & 5170 & 5160 & 5130 & 5120 & 5100 & 5080 & 5070 & 5040 & 5030\end{array}$ $\begin{array}{llllllllllllllll}5010 & 5000 & 4990 & 4980 & 4980 & 4970 & 4970 & 4960 & 4940 & 4920 & 4900 & 4870 & 4840 & 4810 & 4790 & 4770\end{array}$ $4750 \quad 4730 \quad 4700 \quad 4670 \quad 4640 \quad 4610$ 
Table 3.--Listing of data for 1971-77--Continued

Card number

\section{Group III: Array data--Continued Specific yield, dimensionless (Parameter card)}

1505 .15

Group III: Array data--Continued Confining bed thickness at stream nodes, in feet (First card is parameter card)

1506. 1507. 1508. 1509 . 1510 . 1511 . 1512 . 1513 . 1514 . 1515 . 1516 . 1517 . 1518 . 1519 . 1520 . 1521 . 1522 . 1523. 1524 . 1525 . 1526 . 1527. 1528 . 1529 . 1530 . 1531. 1532. 1533. 1534. 1535. 1536. 1537. 1538. 1539 . 1540 . 1541 . 1542 . 1543. 1544. 1545. 1546 . 1547.

$\begin{array}{rl} & 2 \\ 44 & 1.0 \\ 30 & 1.0 \\ 31 & 1.0 \\ 32 & 1.0 \\ 33 & 1.0 \\ 34 & 1.0 \\ 43 & 1.0 \\ 28 & 1.0 \\ 29 & 1.0 \\ 35 & 1.0 \\ 40 & 1.0 \\ 41 & 1.0 \\ 42 & 1.0 \\ 43 & 1.0 \\ 26 & 1.0 \\ 27 & 1.0 \\ 33 & 1.0 \\ 34 & 1.0 \\ 35 & 1.0 \\ 36 & 1.0 \\ 37 & 1.0 \\ 38 & 1.0 \\ 39 & 1.0 \\ 25 & 1.0 \\ 32 & 1.0 \\ 20 & 1.0 \\ 21 & 1.0 \\ 22 & 1.0 \\ 23 & 1.0 \\ 24 & 1.0 \\ 30 & 1.0 \\ 31 & 1.0 \\ 17 & 1.0 \\ 18 & 1.0 \\ 19 & 1.0 \\ 29 & 1.0 \\ 9 & 1.0 \\ 10 & 1.0 \\ 11 & 1.0 \\ 12 & 1.0 \\ 13 & 1.0 \\ & -122- \\ & \end{array}$


Table 3.--Listing of data for 1971-77--Continued

Card

number

1548.

1549 .

1550 .

1551 .

1552.

1553.

1554.

1555 .

1556.

1557.

1558 .

1559.

1560 .

1561 .

1562.

1563.

1564.

1565 .

1566.

1567.

1568 .

1569.

1570 .

1571.

1572 .

1573.

1574.

1575 .

1576.

1577.

1578.

1579 .

1580 .

1581 .

1582 .

1583.

1584.

1585 .

1586 .

1587.

1588.

1589.

1590 .

1591.

1592.

1593.

1594.

1595.

1596.
Group III: Array data--Continued

Confining bed thickness at stream nodes, in feet--Continued

11

11

11

11

12

13

13

13

14

14

28

29

29

30

30

30

31

31

31

31

32

32

33

34

34

35

35

35

35

35

35

35

35

35

35

35

35

36

36

36

36

36

36

37

37

38

39

40

41
14

15

16

28

8

5

6

7

3

4

54

52

53

49

50

51

5

6

7

8

4

9

9

10

14

11

12

13

14

15

16

17

18

19

20

21

22

23

24

25

26

27

28

29

30

31

32

32

32
1.0

1.0

1.0

1.0

1.0

1.0

1.0

1.0

1.0

1.0

1.0

1.0

1.0

1.0

1.0

1.0

1.0

1.0

1.0

1.0

1.0

1.0

1.0

1.0

1.0

1.0

1.0

1.0

1.0

1.0

1.0

1.0

1.0

1.0

1.0

1.0

1.0

1.0

1.0

1.0

1.0

1.0

1.0

1.0

1.0

1.0

1.0

1.0

1.0 
Table 3.--Listing of data for 1971-77--Continued

Card number

1597. 1598. 1599. 1600 . 1601 . 1602 . 1603. 1604. 1605. 1606.

1607. 1608. 1609 . 1610. 1611. 1612 . 1613. 1614. 1615. 1616. 1617. 1618. 1619. 1620. 1621. 1622. 1623. 1624. 1625 . 1626. 1627. 1628. 1629. 1630. 1631. 1632. 1633. 1634. 1635. 1636. 1637. 1638. 1639.

Group III: Array data--Continued

Confining bed thickness at stream nodes, in feet--Continued

$\begin{array}{lll}42 & 32 & 1.0 \\ 43 & 33 & 1.0 \\ 44 & 34 & 1.0 \\ 45 & 35 & 1.0 \\ 46 & 36 & 1.0 \\ 47 & 37 & 1.0 \\ 48 & 38 & 1.0 \\ 49 & 38 & 1.0 \\ 50 & 38 & 1.0 \\ 51 & 38 & 1.0\end{array}$

Group III: Array data--Continued

Initial rate of gain in stream cells, in cubic feet per second (First card is parameter card)

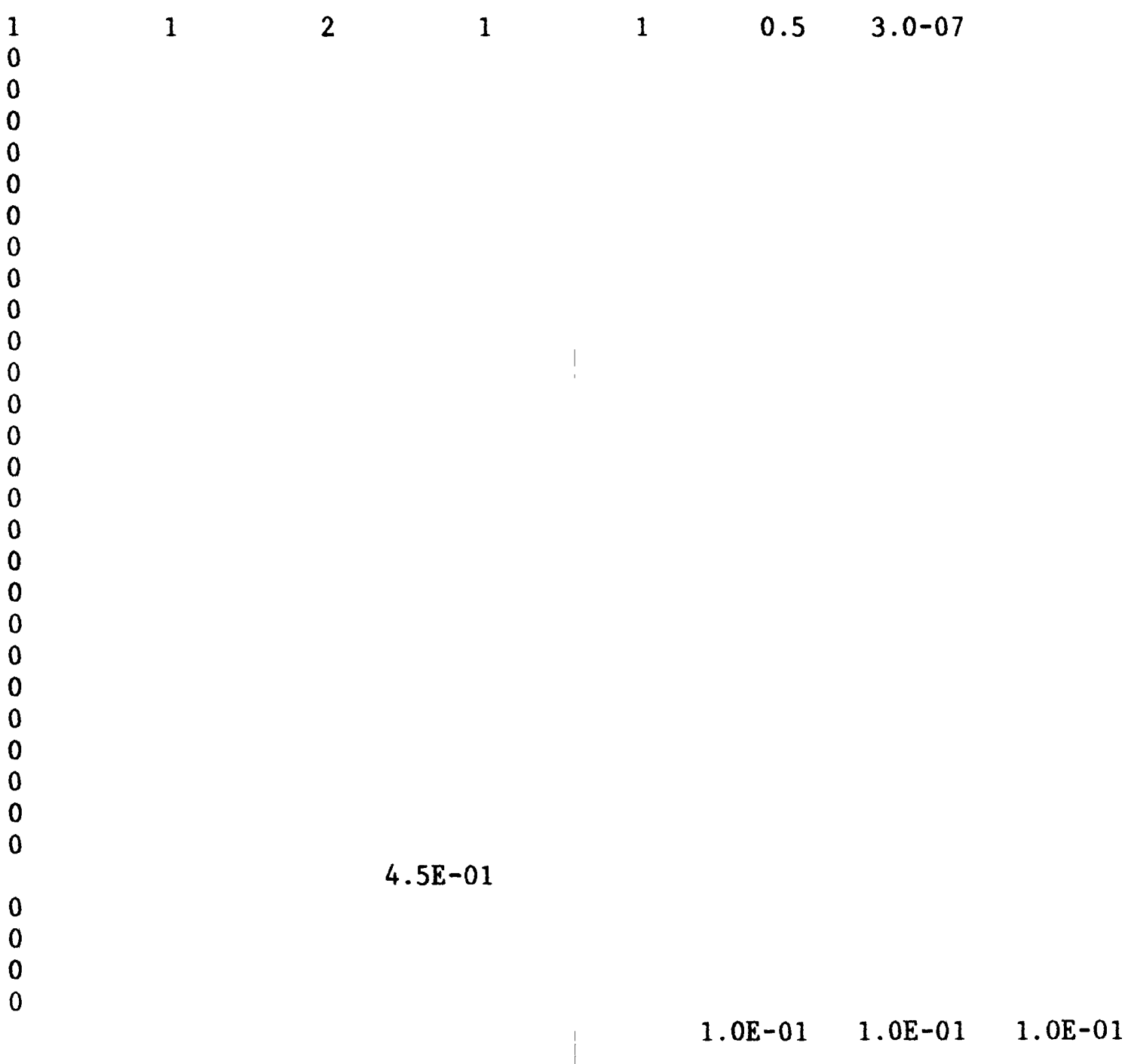




\section{Table 3.--Listing of data for 1971-77--Continued}

Card

Group III: Array data--Continued

number Initial rate of gain in stream cells, in cubic feet per second--Continued

1640 .

1641.

1642.

1643.

1644 .

1645.

1646 .

1647.

1648 .

1649.

1650 .

1651.

1652.

1653.

1654.

1655 .

1656.

1657.

1658 .

1659.

1660.

1661 .

1662 .

1663.

1664.

1665 .

1666.

1667.

1668 .

1669.

1670 .

1671.

1672 .

1673.

1674.

1675.

1676 .

1677.

1678 .

1679.

1680 .

1681.

1682 .

1683.

1684.

1685.

1686.

1687.

1688 .

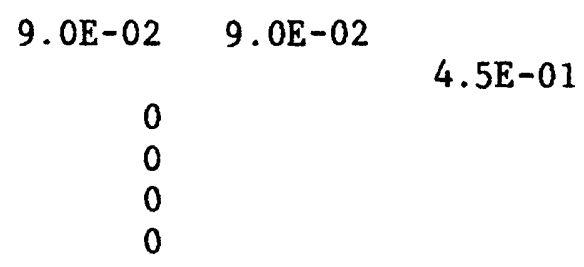

4. $5 \mathrm{E}-01$

0

0

0

0

1. $8 \mathrm{E}-01$

$$
3.0 \mathrm{E}-01 \quad 1.0 \mathrm{E}-01
$$

3. $0 \mathrm{E}-01 \quad 3.0 \mathrm{E}-01$

0

0

0

0

5. $0 \mathrm{E}-01 \quad 3.0 \mathrm{E}-01$

1. $2 \mathrm{E}-01 \quad 1.5 \mathrm{E}-01$

$1.7 \mathrm{E}-01 \quad 3.0 \mathrm{E}-01$

3. $0 \mathrm{E}-01$

$3.0 \mathrm{E}-01$

4. $9 E-01$

$$
6.0 \mathrm{E}-01 \quad 5.5 \mathrm{E}-01
$$

$5.5 \mathrm{E}-01$

5. $0 \mathrm{E}-01$

4. $8 \mathrm{E}-01$

$6.9 \mathrm{E}-01$

$5.0 \mathrm{E}-01$

0
0
0
0
$4.5 \mathrm{E}-01$
0
0
0
0
0
0
0
$3.0 \mathrm{E}-01$

$$
\begin{array}{rrrr}
0 & & \\
0 & & \\
0 & & \\
0 & & \\
0 & & & \\
6.5 \mathrm{E}-01 & 6.5 \mathrm{E}-01 & 6.0 \mathrm{E}-01 & \\
0 & & & \\
0 & & & \\
0 & & & \\
0 & &
\end{array}
$$

$\begin{array}{llllllll}0 & 5.5 \mathrm{E}-01 & 6.7 \mathrm{E}-01 & 9.0 \mathrm{E}-01 & 7.5 \mathrm{E}-01 & 7.5 \mathrm{E}-01 & 7.0 \mathrm{E}-01 & 7.0 \mathrm{E}-01\end{array}$

$1.53 \mathrm{E} 00$

0

0

$3.5 \mathrm{E}-01$ 
Table 3.--Listing of data for 1971-77--Continued

Card number
Group III: Array data--Continued

1689 . 0

1690 .

1691.

1692.

1693.

1694.

1695.

1696.

1697.

1698.

1699.

1700 .

1701.

1702 .

1703.

1704 .

1705 .

1706 .

1707.

1708 .

1709 .

1710 .

1711 .

1712 .

1713.

1714.

1715.

1716 .

1717.

1718.

1719 .

1720 .

1721.

1722 .

1723.

1724.

1725 .

1726 .

1727.

1728.

1729 .

1730 .

1731.

1732 .

1733.

1734.

1735 .

1736 .

1737.
0

0

0

0

0

0

0

0

$9 . \mathrm{OE}-02 \quad 9 . \mathrm{OE}-02$
0

0

0

0

0

0

0

0

0

0

0

0

0

0

0

0

0

0

0

0

0

0

0

0

0

0

0

0

0

0

0

0

0

0

0

0

0

0 
Table 3.--Listing of data for 1971-77--Continued

Card

Group III: Array data--Continued

number Initial rate of gain in stream cells, in cubic feet per second--Continued

1738.

1739.

1740 .

1741 .

1742 .

1743.

1744.

1745 .

1746 .

1747 .

1748 .

1749 .

1750 .

1751 .

1752.

1753.

1754.

1755.

1756.

1757.

1758 .

1759 .

1760 .

1761 .

1762.

1763.

1764 .

1765 .

1766 .

1767.

1768 .

1769 .

1770 .

1771 .

1772.

1773.

1774 .

1775 .

1776.

1777.

1778 .

1779 .

1780 .

1781 .

1782 .

1783.

1784 .

1785 .

1786 .

0

0

0

0

0

0

0

0

0

0

0

0

0

0

0

0

0

0

0

0

0

0

0

0

0

0

0

0

0

0

0

0

0

0

0

0

0

0

0

0

0

0

0

0

0

0

0

0

0 
Table 3.--Listing of data for 1971-77--Continued

Card Group III: Array data--Continued number Initial rate of gain in stream cells, in cubic feet per second--Continued

1787. 1788 . 1789. 1790 . 1791. 1792 . 1793. 1794. 1795. 1796. 1797. 1798. 1799. 1800 . 1801 . 1802 . 1803. 1804 . 1805 . 1806 . 1807 . 1808 . 1809 . 1810 . 1811 .

1812 .

1813. 1814 . 1815 . 1816 . 1817 . 1818 . 1819 . 1820 . 1821 . 1822 . 1823. 1824 . 1825 . 1826 . 1827. 1828 . 1829. 1830 . 1831. 1832. 1833. 1834.

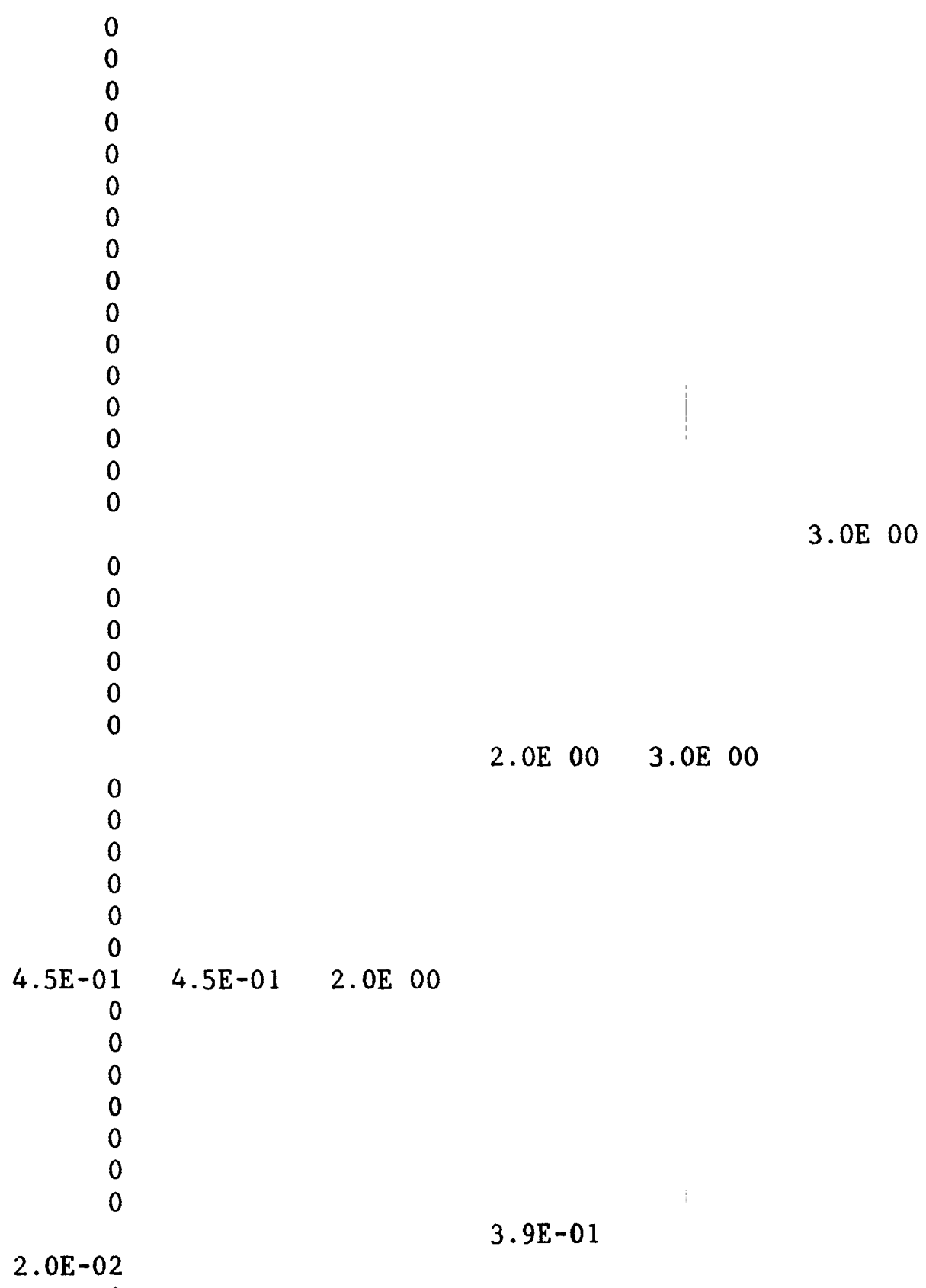

0

0

0

0

0

0

0

0

0

0

0

0

0

0

0

0

0

0

0

0

0

0

0

0

0

0

0

0

$\begin{array}{rrr}4.5 \mathrm{E}-01 & 4.5 \mathrm{E}-01 & 2.0 \mathrm{E} \quad 00\end{array}$

0

0

0

0

0

0

2. $0 \mathrm{E}-02$

$3.9 \mathrm{E}-01$

$2.0 \mathrm{E} \quad 00 \quad 3.0 \mathrm{E} \quad 00$

$3.0 \mathrm{E} 00$ 
Table 3.--Listing of data for 1971-77--Continued

Card Group III: Array data--Continued

number Initial rate of gain in stream cells, in cubic feet per second--Continued

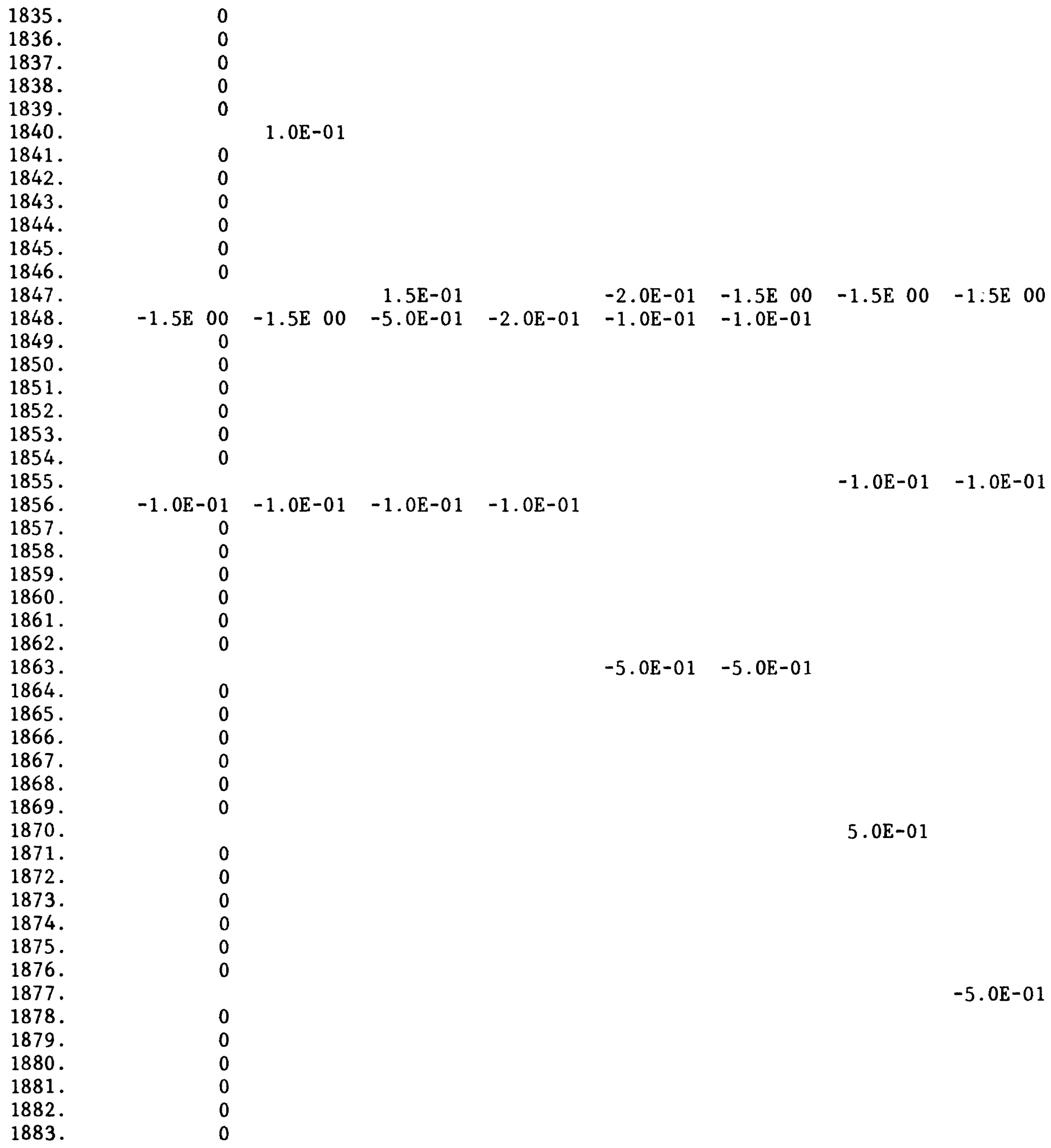
(

(

(1)

0

(1)

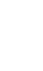$$
\text { (1) }
$$

0

0

0

0

0

0

0

0

0

0

0

0

0

0 0

0

0

0

0

0 
Table 3.--Listing of data for 1971-77--Continued

Card number

1884 .

1885.

1886.

1887.

1888.

1889.

1890 .

1891.

1892.

1893.

1894.

1895.

1896.

1897.

1898.

1899.

1900.

1901.

1902.

1903.

1904.

1905.

1906.

1907.

1908.

1909.

1910.

1911.

1912 .

1913.

1914.

1915.

1916.

1917.

1918.

1919 .

1920 .

1921.

1922.

1923.

1924.

1925.

1926.

1927.

1928.

1929.

1930.

1931.

1932.
Group III: Array data--Continued

Initial rate of gain in stream cells, in cubic feet per second--Continued

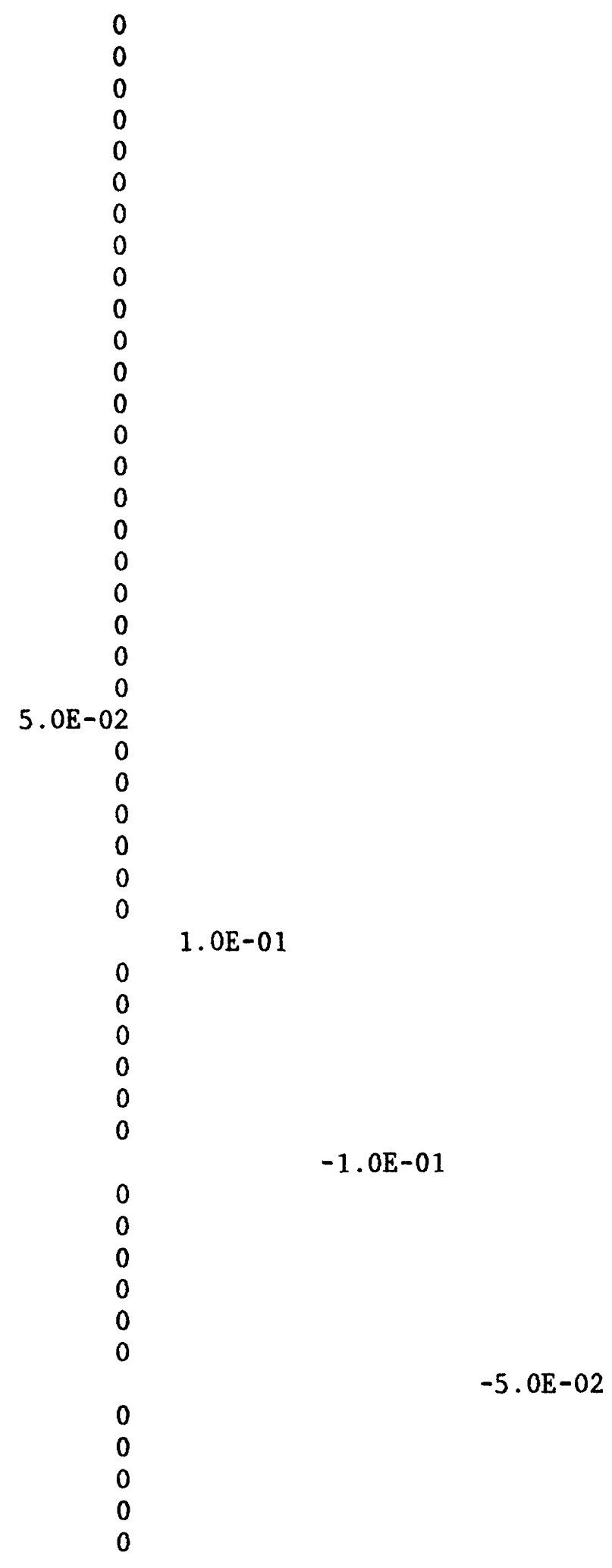


Table 3.--Listing of data for 1971-77--Continued

Card Group III: Array data--Continued number Initial rate of gain in stream cells, in cubic feet per second--Continued 1933. 0

1934.0

$1935 . \quad 0$

1936. 0

1937.0

$1938 . \quad 0$

$1939 . \quad 0$

$1940 . \quad 0$

1941 . 0

1942 . 0

$1943 . \quad 0$

$1944 . \quad 0$

1945 . 0

$1946 . \quad 0$

1947.0

1948 . 0

$1949 . \quad 0$

$1950 . \quad 0$

1951 . 0

1952 . 0

$1953 . \quad 0$

1954 . 0

1955.0

1956 . 0

1957.0

1958 . 0

1959 . 0

1960.0

1961 . 0

1962 . 0

$1963 . \quad 0$

1964 . 0

1965 . 0

1966.

1967.

1968 . 0

$1969 . \quad 0$

1970 . 0

1971.

Group III: Array data--Continued

Head at stream nodes, in feet

(Parameter card)

1972.

0

0

2 
Card number

1973.
Group III: Array data--Continued Top of aquifer, altitude of stream, in feet (Parameter card)

0

$$
2
$$

Group III: Array data--Continued Recharge rate, in feet per second (Parameter card)

1974.

$2.19 E-09$

Number of streams and node identification

1975.

1976.
$\begin{array}{lllll}10 & 15 & 20 & 30 & 35\end{array}$

Test card to calculate initial recharge/discharge at constant-flux boundary nodes

1977

0

0

Group IV: Data that change with pumping period

1978. 2 1 224 2555 1000

1.5 24

Parameters for streamflow accounting procedure

\section{Number of streams}

1979

5

\begin{tabular}{|c|c|c|c|c|c|c|}
\hline & Stream & Upstream & node & $\begin{array}{l}\text { Inflow } \\
\text { rate, in } \\
\text { cubic feet }\end{array}$ & don & $\begin{array}{l}t \\
\text { ream } \\
\text { e }\end{array}$ \\
\hline & identification & $\mathrm{I}$ & $\mathrm{J}$ & per second & $\mathrm{I}$ & $\mathrm{J}$ \\
\hline $\begin{array}{l}1980 . \\
1981 . \\
1982 . \\
1983 . \\
1984 .\end{array}$ & $\begin{array}{l}10 \\
15 \\
20 \\
30 \\
35\end{array}$ & $\begin{array}{l}14 \\
11 \\
30 \\
32 \\
34\end{array}$ & $\begin{array}{r}3 \\
28 \\
49 \\
4 \\
14\end{array}$ & $\begin{array}{r}.39 \\
11.0\end{array}$ & $\begin{array}{r}4 \\
7 \\
28 \\
46 \\
34\end{array}$ & $\begin{array}{l}44 \\
35 \\
54 \\
36 \\
14\end{array}$ \\
\hline
\end{tabular}


Table 3.--Listing of data for 1971-77--Continued

Card

number

1985.

1986.

1987.

1988.

1989.

1990.

1991.

1992.

1993.

1994.

1995.

1996.

1997.

1998.

1999.

2000 .

2001 .

2002 .

2003.

2004 .

2005 .

2006 .

2007 .

2008 .

2009 .

2010.

2011.

2012.

2013.

2014.

2015 .

2016.

2017.

2018.

2019 .

2020 .

2021 .

2022.

2023.

2024.

2025 .

2026.

2027.

2028 .

2029 .

2030 .

2031.

2032.

2033.
Group IV: Data that change with pumping period--Continued $I, J$, and pumping rate, in cubic feet per second
$25-0.0331507$
$37-0.4409041$
$31-0.4770384$
$33-0.0947636$
$45-0.3275287$
$31-0.4056222$
$34-0.4100266$
$37-0.3354849$
$38-0.1776876$
$43-0.0757730$
$45-0.5163456$
$46-0.2252546$
$33-0.0639$
$47-0.5199211$
$32-0.1444423$
$33-0.1444423$
$43-0.0344530$
$45-0.0871389$
$46-0.2247616$
$47-0.7840374$
$48-0.1860464$
$19-0.1148671$
$41-0.1178269$
$42-0.3322645$
$43-0.7644548$
$45-0.2240986$
$47-0.3770654$
$48-0.3718086$
$38-0.4289935$
$41-0.2735642$
$42-0.1765984$
$43-0.2081911$
$47-0.3652021$
$48-0.4817505$
$39-0.5114677$
$41-0.2734931$
$42-0.4837158$
$47-0.0640281$
$10-0.6166027$
$17-0.0309012$
$37-0.1989040$
$42-0.4140520$
$47-0.1335973$
$22-0.1498885$
$29-0.0981734$
$31-0.1190583$
$47-0.4397438$
2051633
39

8

9

14

14

15

15

15

15

15

15

15

30

15

16

16

16

16

16

16

16

17

17

17

17

17

17

17

18

18

18

18

18

18

19

19

19

19

20

20

20

20

20

21

21

21

21

22

22

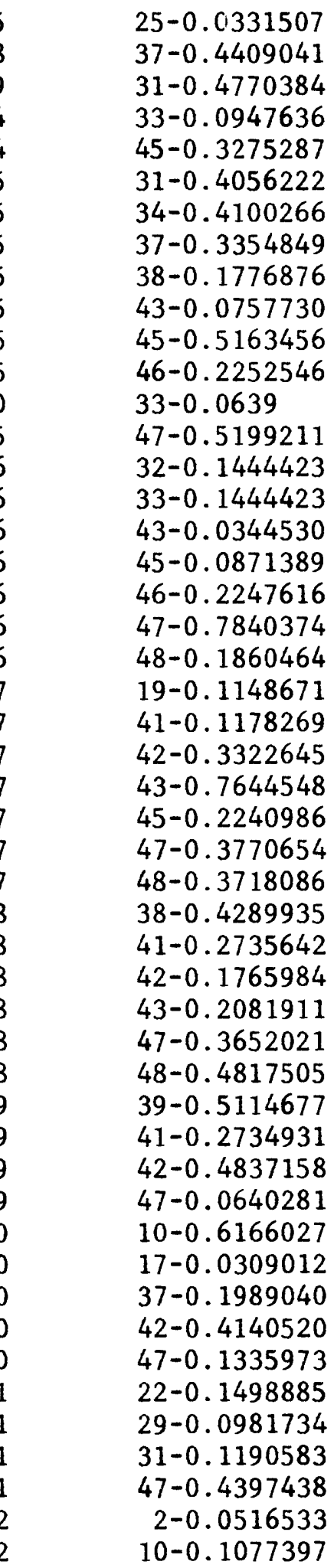


Table 3.--Listing of data for 1971-77--Continued

Card number

2034.

2035.

2036.

2037.

2038.

2039.

2040.

2041.

2042.

2043.

2044 .

2045.

2046.

2047.

2048 .

2049 .

2050 .

2051 .

2052.

2053.

2054.

2055 .

2056.

2057.

2058.

2059.

2060 .

2061 .

2062.

2063.

2064.

2065 .

2066 .

2067.

2068.

2069.

2070 .

2071.

2072.

2073.

2074 .

2075.

2076 .

2077.

2078.

2079.

2080 .

2081 .

2082 .

Group IV: Data that change with pumping period--Continued $\mathrm{I}$, $\mathrm{J}$, and pumping rate, in cubic feet per second--Continued

22

22

22

22

22

22

22

23

23

23

23

23

23

23

23

24

24

24

24

24

25

25

25

25

25

25

25

25

26

26

26

26

27

27

27

27

27

27

27

28

28

28

28

28

28

29

29

29

29
$15-0.2339965$

16-0.3028315

19-0.1263514

32-0.0331506

35-0.1735201

36-0.3237164

39-0. 1013464

2-0.0454702

9-0.2320548

$11-0.0994520$

17-0.1727624

$31-0.1577972$

34-0.0412963

40-0.1549321

41-0.2913471

2-0.0166388

12-0. 1989041

27-0.0910696

38-0.0118395

41-0.0467425

2-0.0715493

10-0.0140654

26-0.2366011

27-0. 2928626

34-0.0331507

36-0.0576822

44-0. 2048712

45-0.6892027

2-0.0040481

$34-0.1277722$

44-0.2942123

45-0.2265849

2-0. 1584384

3-0. 1203213

28-0. 1824945

30-0.0927035

32-0.7337904

45-0.3392972

46-0.6494219

27-0.5809658

30-0. 1469049

33-0. 2635479

40-0.2287397

47-0.3305123

48-1.0294943

14-0.0282728

25-0. 1009675

26-0. 1330289

33-0.0890806 
Table 3.--Listing of data for 1971-77--Continued

Card number

2083. 2084 . 2085. 2086. 2087. 2088. 2089 . 2090 . 2091. 2092. 2093. 2094. 2095 . 2096. 2097. 2098. 2099. 2100 . 2101 . 2102 . 2103 . 2104 . 2105 . 2106. 2107 . 2108 . 2109 . 2110 . 2111. 2112 . 2113. 2114 . 2115 . 2116 . 2117 . 2118 . 2119 . 2120 . 2121 . 2122. 2123. 2124 . 2125 . 2126 . 2127 . 2128 . 2129 . 2130 . 2131 .
Group IV: Data that change with pumping period--Continued $I$, $J$, and pumping rate, in cubic feet per second--Continued

$\begin{array}{lr}29 & 37-0.2370274 \\ 29 & 45-0.7218561 \\ 29 & 46-0.6865506 \\ 29 & 47-0.2920575 \\ 30 & 7-0.3471546 \\ 30 & 29-0.0595292 \\ 30 & 30-0.1456735 \\ 30 & 36-0.2640452 \\ 30 & 37-0.6001931 \\ 30 & 45-0.2552602 \\ 30 & 46-0.1682397 \\ 30 & 47-0.0828767 \\ 30 & 48-0.7172151 \\ 31 & 6-0.4111289 \\ 31 & 7-0.9736999 \\ 31 & 29-0.0322746 \\ 31 & 33-0.1886274 \\ 31 & 37-0.3421150 \\ 31 & 42-0.2532712 \\ 31 & 38-0.2923298 \\ 31 & 44-0.7095430 \\ 31 & 45-0.3751000 \\ 31 & 46-0.7200329 \\ 31 & 47-0.6301945 \\ 31 & 48-0.4456328 \\ 32 & 7-0.3363960 \\ 32 & 23-0.1788716 \\ 32 & 33-0.2221096 \\ 32 & 37-0.2151479 \\ 32 & 38-0.1276301 \\ 32 & 39-0.2081863 \\ 32 & 40-0.6647422 \\ 32 & 42-0.4530277 \\ 32 & 43-0.3754551 \\ 32 & 44-0.2652054 \\ 32 & 45-0.5987014 \\ 32 & 46-1.0589981 \\ 33 & 6-0.3172117 \\ 33 & 7-0.0908900 \\ 33 & 14-0.0331507 \\ 33 & 23-0.1035959 \\ 33 & 26-0.0907381 \\ 33 & 40-0.1980753 \\ 33 & 41-0.3550438 \\ 33 & 42-0.1989041 \\ 33 & 43-0.1574657 \\ 33 & 44-0.2264192 \\ 33 & 45-0.5184767 \\ 33 & 46-1.2240887\end{array}$


Card number

2132 . 2133. 2134 . 2135 . 2136 . 2137 . 2138 . 2139 . 2140 . 2141 . 2142 . 2143. 2144 . 2145 . 2146 . 2147 . 2148. 2149 . 2150 . 2151 . 2152 . 2153. 2154 . 2155 . 2156 . 2157 . 2158 . 2159 . 2160 . 2161 . 2162 . 2163. 2164 . 2165 . 2166 . 2167 . 2168 . 2169 . 2170 . 2171. 2172 . 2173. 2174 . 2175 . 2176 . 2177 . 2178 . 2179 . 2180 .
Group IV: Data that change with pumping period--Continued $I, J$, and pumping rate, in cubic feet per second--Continued

$\begin{array}{lr}33 & 47-0.1873013 \\ 34 & 5-0.0024123 \\ 34 & 6-0.2815926 \\ 34 & 7-0.1650465 \\ 34 & 34-0.3068095 \\ 34 & 41-0.1376939 \\ 34 & 42-0.7775493 \\ 34 & 43-0.4641096 \\ 34 & 45-0.2787972 \\ 34 & 46-0.6938438 \\ 35 & 5-0.2143431 \\ 35 & 6-0.0305242 \\ 35 & 24-0.1752250 \\ 35 & 39-0.2773054 \\ 35 & 40-0.3112849 \\ 35 & 41-0.0727657 \\ 35 & 45-0.2068602 \\ 35 & 46-0.4173671 \\ 36 & 6-0.1553295 \\ 36 & 27-0.1190583 \\ 36 & 33-0.1226575 \\ 36 & 35-0.2591673 \\ 36 & 42-0.3152630 \\ 36 & 43-0.3412863 \\ 36 & 44-0.2068602 \\ 36 & 45-0.3169205 \\ 37 & 5-0.1209623 \\ 37 & 6-0.1343807 \\ 37 & 30-0.1334314 \\ 37 & 42-0.3743659 \\ 37 & 44-0.7533493 \\ 37 & 45-0.7314699 \\ 38 & 7-0.1659810 \\ 38 & 31-0.0485657 \\ 38 & 45-0.3612714 \\ 39 & 6-0.3565062 \\ 39 & 7-0.1782531 \\ 39 & 27-0.1568027 \\ 39 & 31-0.0536575 \\ 39 & 37-0.5237808 \\ 39 & 42-0.4901566 \\ 39 & 43-0.0697822 \\ 39 & 44-0.2645425 \\ 40 & 29-0.2181319 \\ 40 & 31-0.2295921 \\ 40 & 36-0.2148164 \\ 40 & 42-0.2630743 \\ 40 & 45-0.9241936 \\ 41 & 28-0.1463366\end{array}$


Table 3.--Listing of data for 1971-77--Continued

Card number

2181. 2182 . 2183. 2184 . 2185 . 2186 . 2187. 2188 . 2189. 2190 . 2191. 2192. 2193. 2194. 2195. 2196. 2197. 2198. 2199. 2200 . 2201. 2202. 2203. 2204. 2205. 2206. 2207. 2208.
Group IV: Data that change with pumping period--Continued $I, J$, and pumping rate, in cubic feet per second--Continued
$29-0.4867468$

$30-0.1554530$

$31-0.1856912$

$35-0.2461438$

$36-1.259726$

$37-0.2018876$

$38-0.1955322$

42-0.3104324

$45-0.8116943$

$29-0.3773457$

$30-0.1542194$

$31-0.6105883$

$32-0.3477270$

34-0.4470370

35-0. 1326027

36-0.8922981

37-0.2479434

45-0.2201205

29-0. 1121914

30-0.4440297

31-0.8723129

33-0.0752602

35-0.2128274

37-0.3091301

38-0.6623507

39-0.6354986

45-0.2096781

46-0.2105069 